\title{
Design and Analysis of Novel Metallic Structural Concepts for Lightweight Freight Railcars
}

by

Danial Molavitabrizi

A thesis submitted to the Faculty of Graduate and Postdoctoral Affairs in partial fulfillment of the requirements for the degree of

Master of Applied Science

in

Mechanical Engineering

Carleton University

Ottawa, Ontario

(C) 2018, Danial Molavitabrizi 


\section{Abstract}

The Government of Canada is committed to reduce the country's greenhouse gas emission to a certain amount by the year 2020. For that, Transport Canada is investing on research projects focused on developing new energy saving technologies. This research was funded by Transport Canada's Clean Rail Academic Grant Program to develop new methods for reducing the weight of the railcars, especially freight railcars. This can ultimately lead to reduction in the total $\mathrm{CO}_{2}$ that is being produced by the Canadian transport sector.

A typical hopper railcar was selected for the case study in this thesis. The solid model of the railcar was created using CATIA, and a preliminary stress analysis was conducted to illustrate the highly stressed areas of the railcar body. Material selection process was done using CES Software, and a third generation aluminum-lithium alloy, i.e. Al 2099, was selected as an alternative to conventional steel. The new material led to $66 \%$ weight reduction on the body as compared to conventional steel railcar bodies. Then, a sandwich panel was developed and optimized for the floor panel made of the newly selected material. By that, a $12.5 \%$ weight reduction can be achieved on the body made of Al 2099. Finally, the proposed design was validated using finite element simulations and by comparing their results with the exact analytical solutions. 


\section{Acknowledgements}

I would like to sincerely thank professor Jeremy Laliberte for supervising my master thesis. He thought me how to develop my own research path, rather than dictating

one to me. It would not have been possible to finish this project without his support and commitment. I will always appreciate his help.

I would like to thank Transport Canada's Clean Rail Academic Grant for funding my research.

Last but not least, I want to thank my parents for their support. They were always available for me. I can never thank them enough for their help and support. 


\section{Table of Contents}

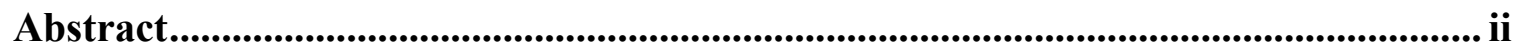

Acknowledgements ............................................................................................................................. iii

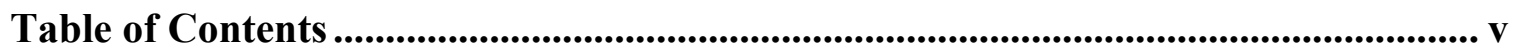

List of Tables ............................................................................................................................... ix

List of Figures.......................................................................................................................... $\mathrm{x}$

Nomenclature .......................................................................................................................... Xv

Chapter 1. Literature Review ............................................................................................. 1

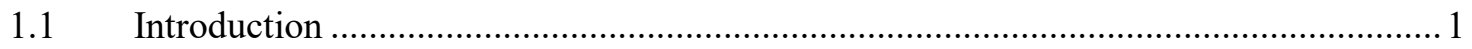

$1.2 \quad$ Thesis Motivation ..............................................................................................

$1.3 \quad$ Weight Reduction Strategies ………………………........................................

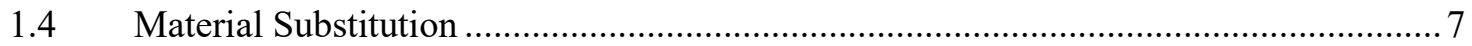

1.4.1 Introduction to Composite Materials....................................................................

1.4.2 Prior Case Studies on Lightweight Railcars............................................................ 10

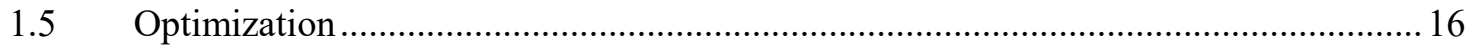

1.5.1 Introduction to Design Optimization......................................................................... 16

1.5.2 Dynamic Behavior and Crashworthiness ………………………………………..... 19

1.6 Previous Studies on Light Weight Design of Railcars ................................................22

1.7 Review of The Canadian Rail System Operation ....................................................29

$1.8 \quad$ Background Summary …………………………………………………………. 31

Chapter 2. Model Preparation and Stress Analysis ...................................................... 33

2.1 Structural Components and Terminology …………………………………………..... 33

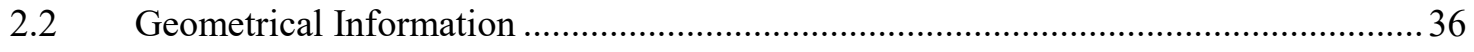

2.3 Development of the CAD Model............................................................................. 39 


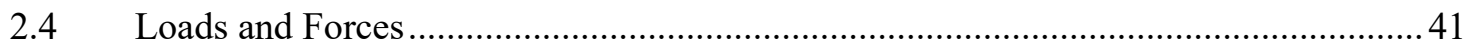

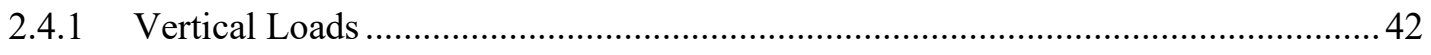

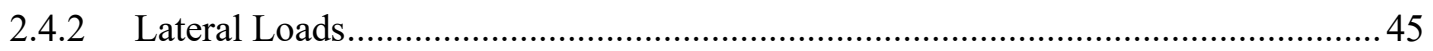

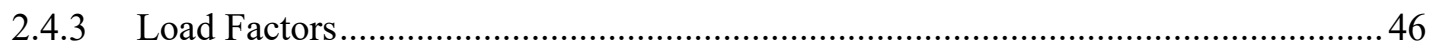

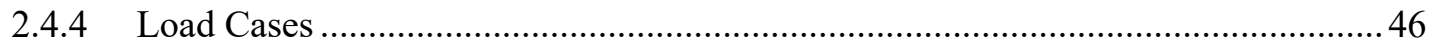

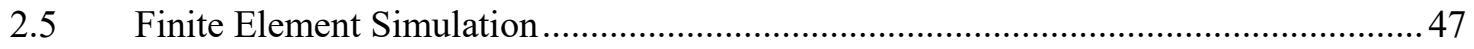

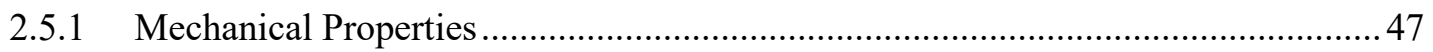

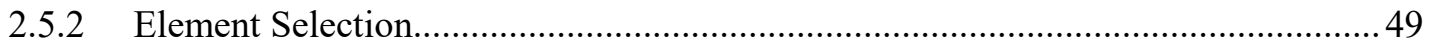

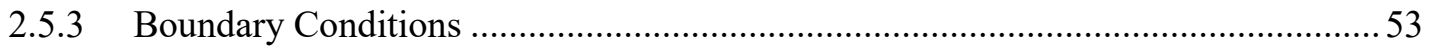

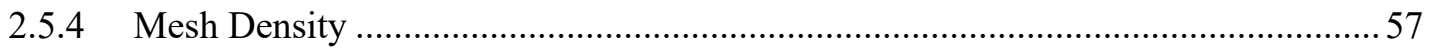

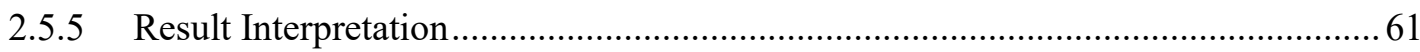

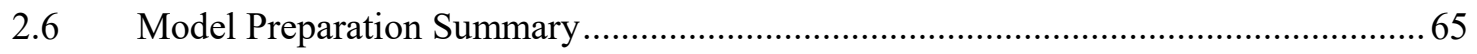

Chapter 3. Material Selection ........................................................................................... 68

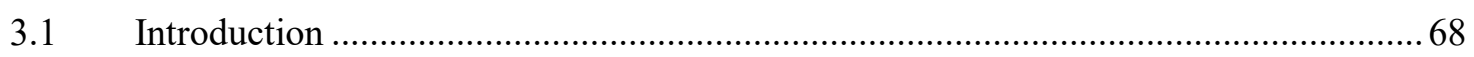

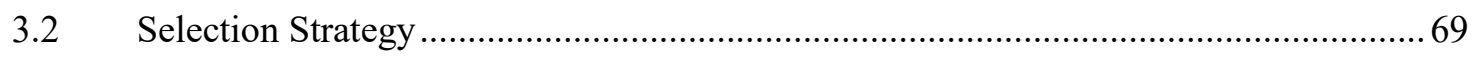

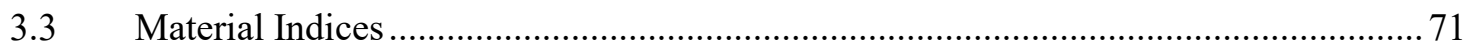

3.4 Material Selection for Covered Hopper Railcars................................................. 72

3.4.1 Functional Requirements in Material Selection of Hopper Cars............................. 73

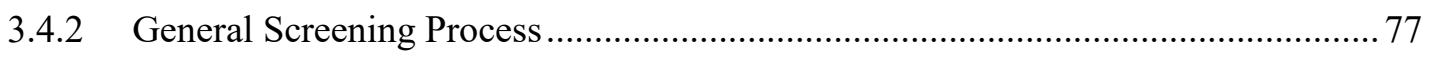

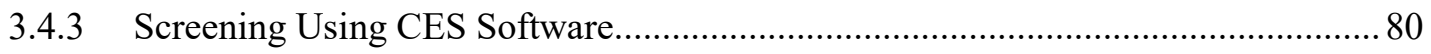

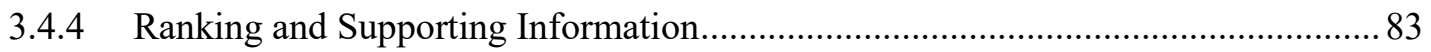

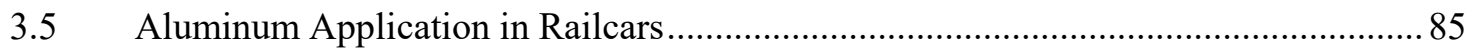

3.5.1 Challenges with 2xxx and 7xxx Series Aluminum Alloys for Rail Applications .... 90

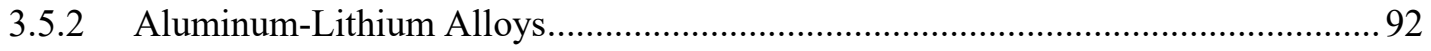

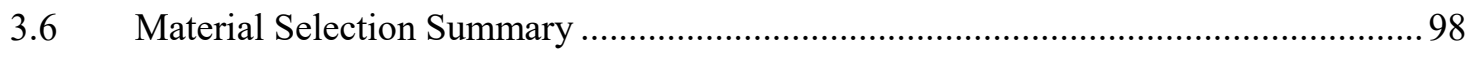


Chapter 4. Structural Optimization .............................................................................. 104

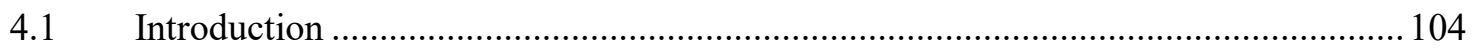

4.2 Types of Cellular Core ………………………………....................................... 109

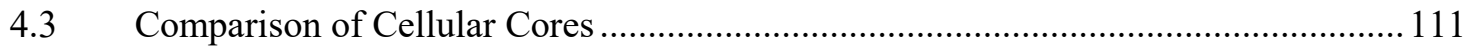

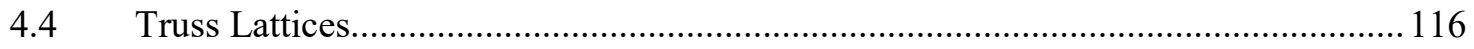

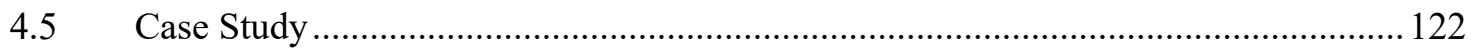

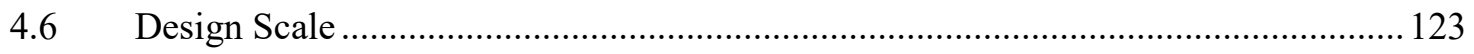

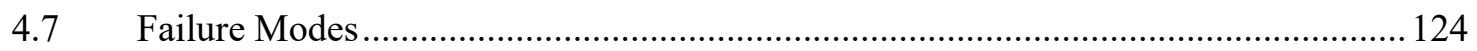

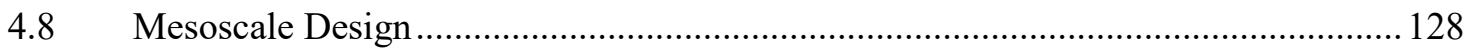

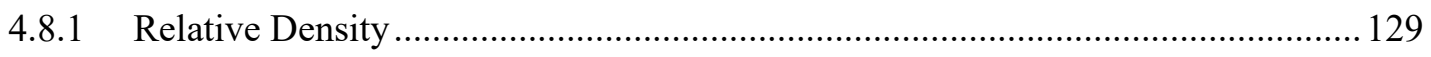

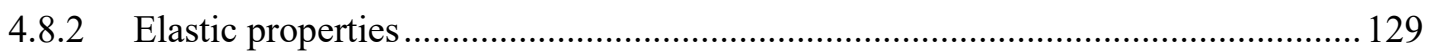

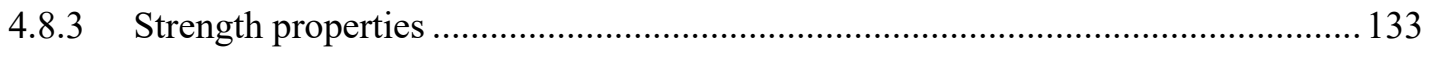

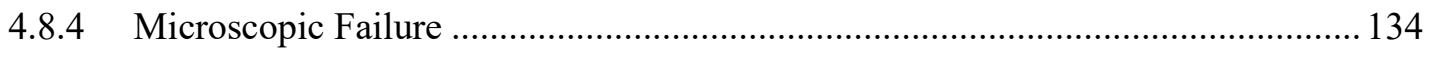

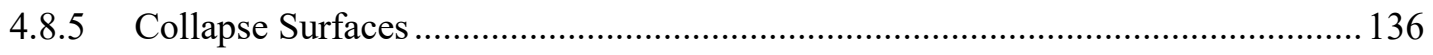

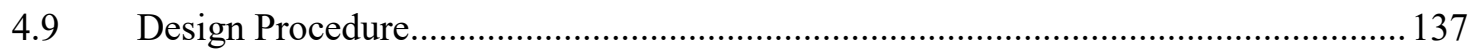

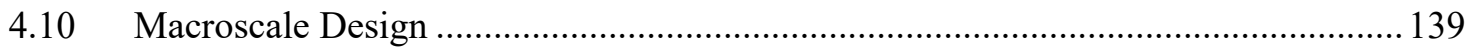

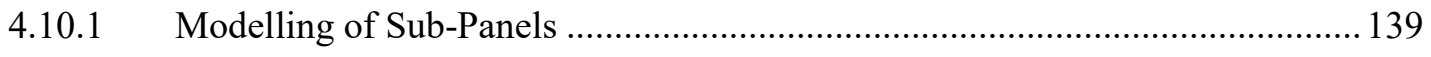

4.10.2 Stress Distribution and Analysis Method …………………………………..... 141

4.10.3 Failure Constraints................................................................................. 142

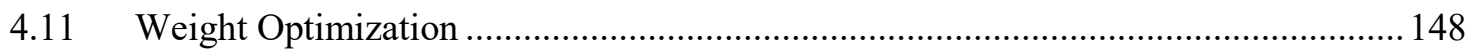

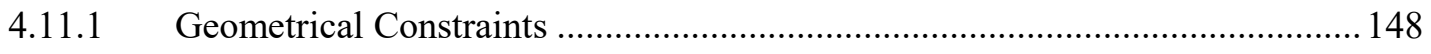

4.11.2 Optimization Problem..................................................................................... 149

Chapter 5. Validation.............................................................................................. 154

$5.1 \quad$ Finite Element Modeling of Sandwich Structures................................................... 154

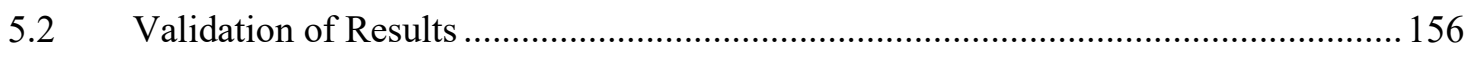

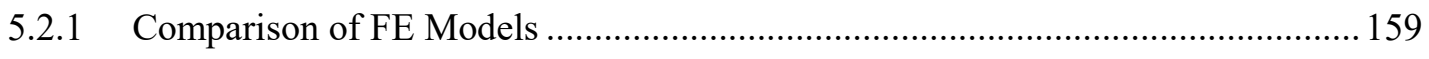




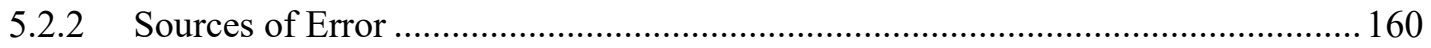

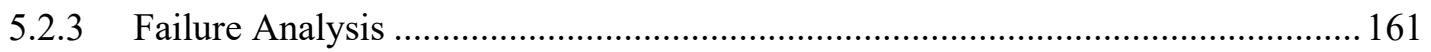

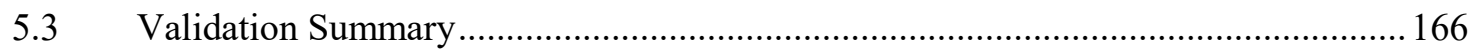

Chapter 6. Conclusions and Recommendations ................................................ 168

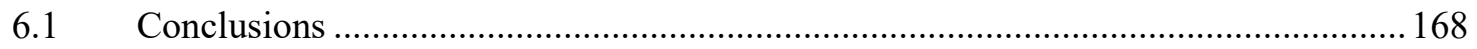

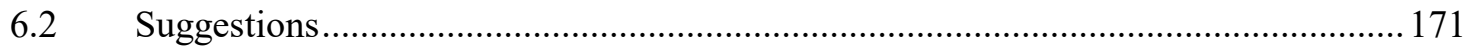

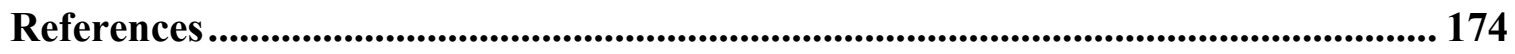




\section{List of Tables}

Table 1: List of some AAR freight car types and their corresponding codes [3] ............ 30

Table 2: Detailed geometrical information of BNSF covered hopper car [24] ............... 36

Table 3: Classification of railcars based on their nominal capacity [6]......................... 43

Table 4: Capacity related info on the selected BNSF railcar [25] ............................... 43

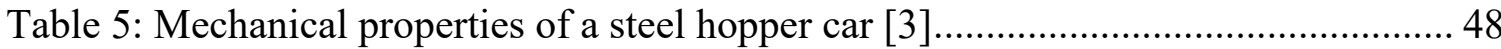

Table 6: Parameters for the hopper car material selection [33] ................................... 73

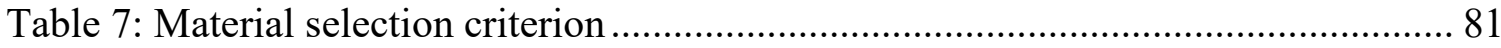

Table 8: Mechanical and thermal properties of the candidate materials $[35,36] \ldots \ldots \ldots . . .83$

Table 9: Mechanical properties of selected wrought aluminum alloys $[42,43] \ldots \ldots \ldots \ldots . . .90$

Table 10: Mechanical properties of Al-Li alloy 2099 and $2055[53,54] \ldots \ldots \ldots \ldots \ldots \ldots \ldots . . . . . . . . . . . .66$

Table 11: Comparison of Aluminum Alloys ........................................................... 101

Table 12: Comparison of Conventional Aluminum Alloys, Third-Generation Al-Li

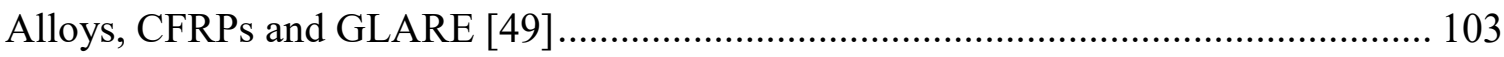

Table 13: Weight saving and structural performance potential of sandwich panel ........ 107

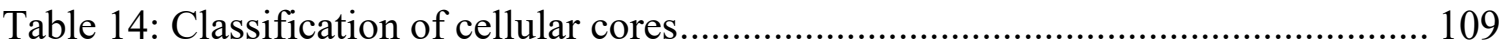

Table 15: Governing equations for bending dominated and stretch dominated lattices [61]

Table 16: Material properties for face sheets and the base material that the lattice is made

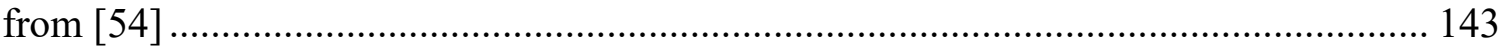

Table 17: Analytical results vs FE Analysis ....................................................... 159 


\section{List of Figures}

Figure 1: Global share of largest $\mathrm{CO}_{2}$ producing countries in 2016 [1] .......................... 1

Figure 2: $\mathrm{CO}_{2}$ emissions in the top 5 emitting countries and European Union [2] ............ 2

Figure 3: $\mathrm{CO}_{2}$ emission per capita in top 6-10 emitting countries [2] ............................. 2

Figure 4: Canada's emission breakdown from 1991 to 2015 [4]...................................... 3

Figure 5: Research path taken to identify areas of interest........................................... 6

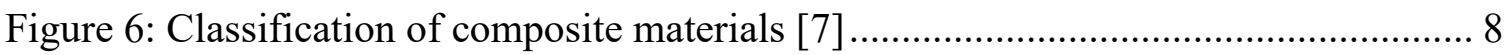

Figure 7: Application of composite materials according to estimates for the year 2000 [7]

Figure 8: Application of carbon composite materials in the year 2015 [8] ..................... 10

Figure 9: Costs vs Energy consumption [9]......................................................... 12

Figure 10: Life cycle energy savings from replacement of steel with aluminum and composites for trucks [10] ........................................................................... 14

Figure 11: Life cycle energy savings from replacement of steel with aluminum and composites for buses [10] .............................................................................. 15

Figure 12: A sizing structural optimization problem [12] ........................................... 17

Figure 13: A shape optimization problem [12] ...................................................... 18

Figure 14: Topology optimization of (a) discrete system, and (b) continuous system [12]

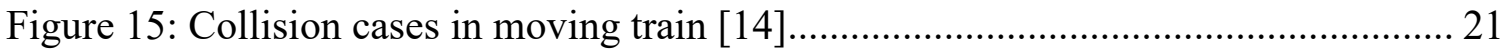

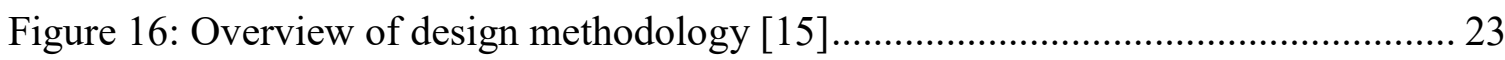

Figure 17: FE model of the boxcar end structure [21] ................................................... 27 
Figure 18: An average weekly number of freight cars on line for $\mathrm{CN}$ railways between

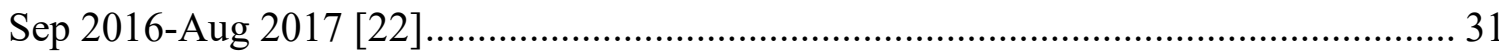

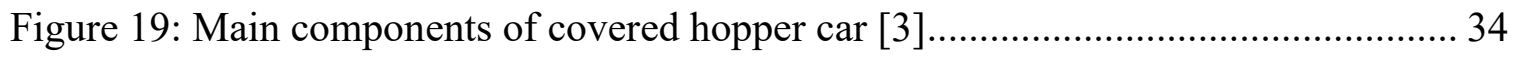

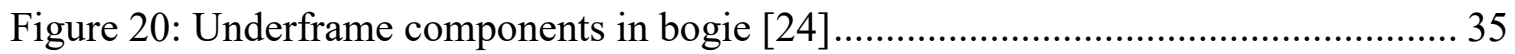

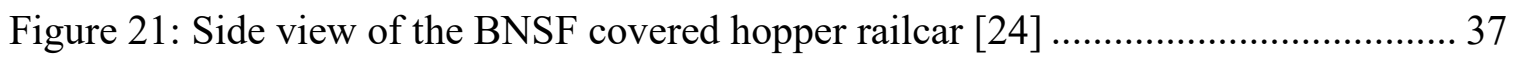

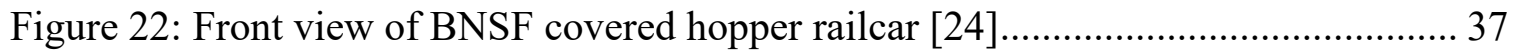

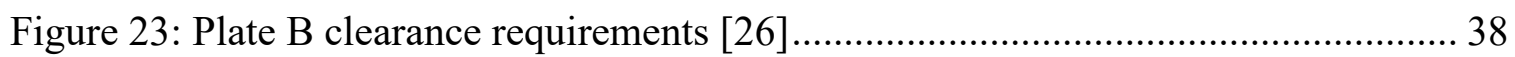

Figure 24: CAD model of the BNSF covered hopper railcar body ............................... 39

Figure 25: Inside view of the BNSF covered hopper railcar body ............................... 40

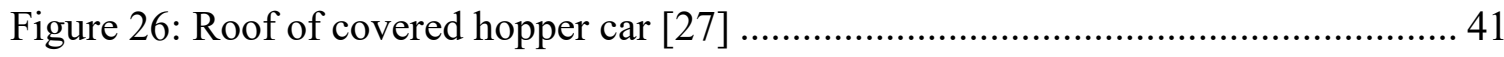

Figure 27: Uniform full capacity load distribution in a box car [6] ............................. 42

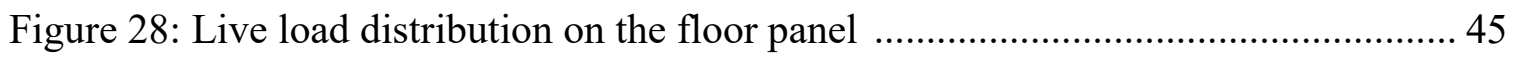

Figure 29: Engineering stress-strain curve of the unknown sample steel [3] ................. 48

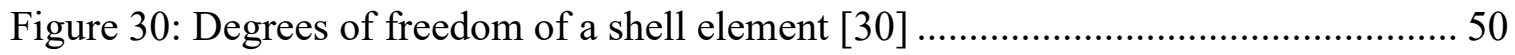

Figure 31: Possible locations of integration points in triangular elements [30] .............. 52

Figure 32: Deformation of square elements into an hourglass mode [30]..................... 52

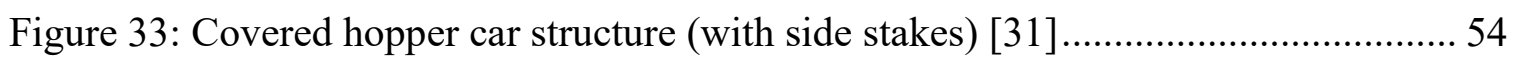

Figure 34: Covered hopper car structure (without side stakes) [31] ............................. 55

Figure 35: Symmetric boundary conditions ....................................................... 56

Figure 36: Center plate and side bearings boundary condition with their respective

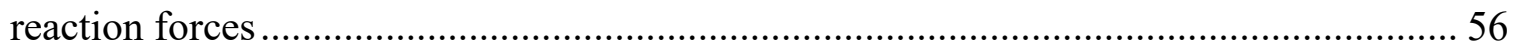

Figure 37: Joint geometry and recommended mesh size [6] .................................. 58

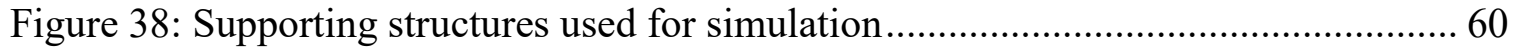




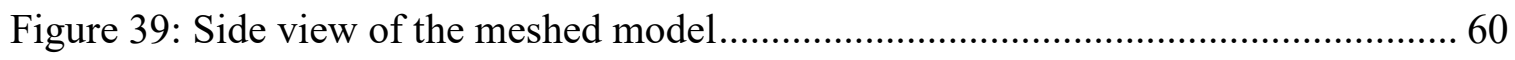

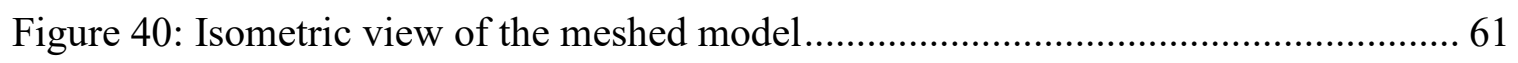

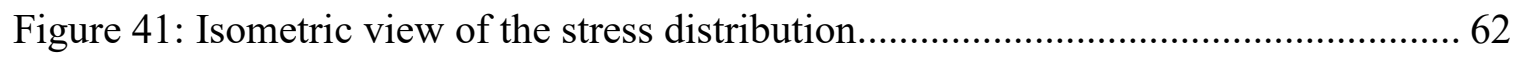

Figure 42: The point that stress is locally concentrated and not shared with neighbor

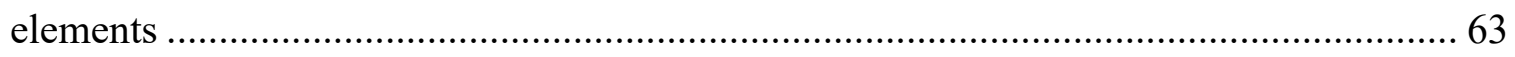

Figure 43: Stress concentration in points where floor stiffeners attached to the edge ..... 64

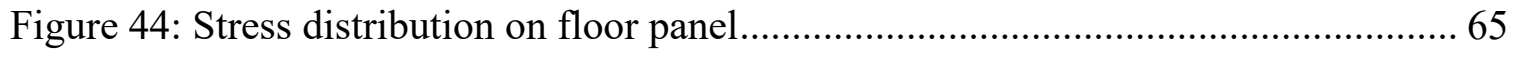

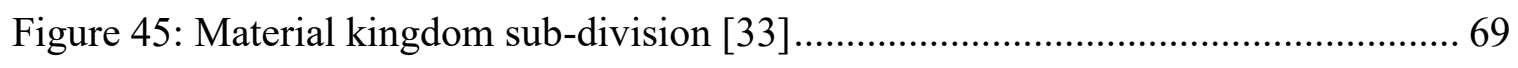

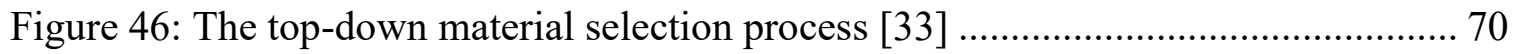

Figure 47: A panel loaded in bending by a force F per unit width [34] ….................... 75

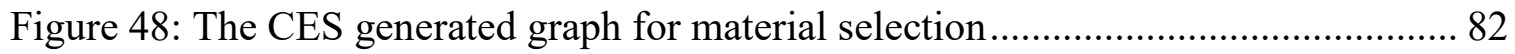

Figure 49: Ultimate tensile $\left(\sigma_{\mathrm{u}}\right)$ and yield strength $\left(\sigma_{\mathrm{y}}\right)$ of 8090 and 2090 Al-Li alloys as a

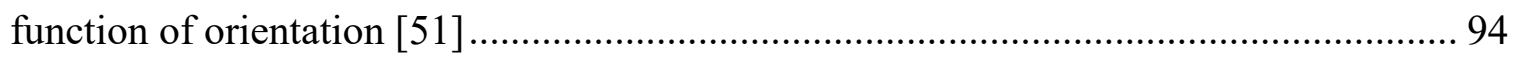

Figure 50: Confidence limit in MMPDS statistical data [56] ................................... 98

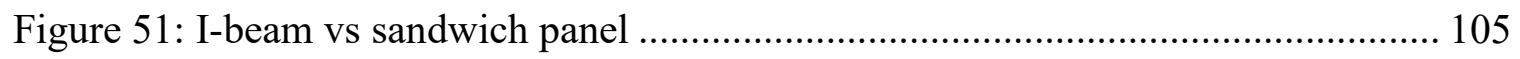

Figure 52: Schematic diagram cross-sectional view in (a) normal panel and (b) sandwich

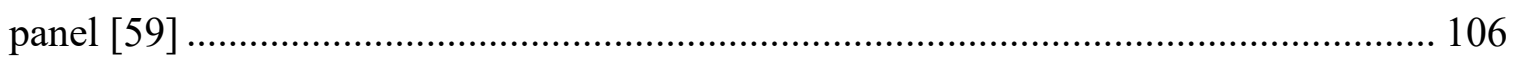

Figure 53: Relative modulus vs relative density on logarithmic scales for cellular

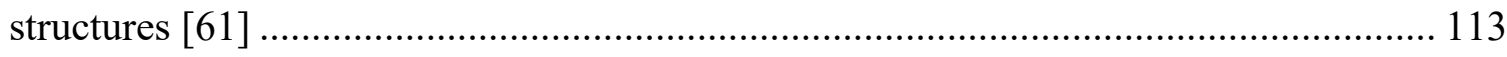

Figure 54: Relative strength vs relative density on logarithmic scales for cellular

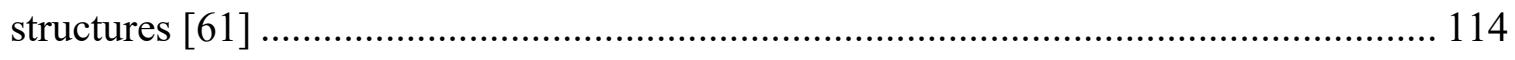

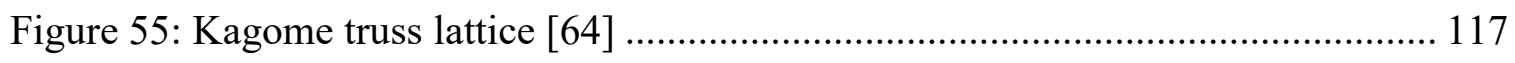

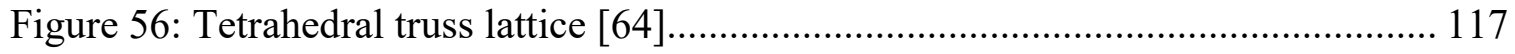




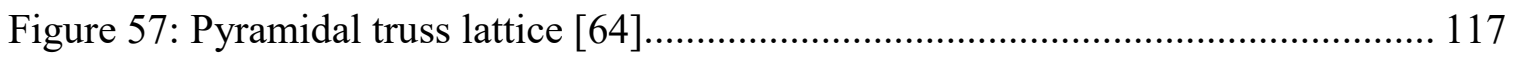

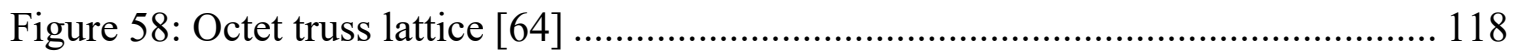

Figure 59: Schematic of the manufacturing process of the tetrahedral lattice truss cores [64] 118

Figure 60: Schematic of the manufacturing process of the pyramidal lattice truss cores $[66]$ 119

Figure 61: Schematic of the manufacturing process of the octet lattice truss cores [68] 121 Figure 62: Stress distribution in sandwich structure as a result of uniform lateral load [71]

Figure 63: Sandwich failure modes [72] .............................................................. 127

Figure 64: The unit cell of an octet-truss lattice [68]............................................... 128

Figure 65: Engineering shear stress versus average shear stress [75] ......................... 130

Figure 66: The schematic of the sandwich panel design process ................................. 138

Figure 67: Schematic of the whole floor panel with its stiffeners [76] ......................... 139

Figure 68: Schematic of the sample sub-panel [74]............................................... 140

Figure 69: $\mathrm{K}_{2}$ for determining face sheet stress in a flat rectangular sandwich panel with isotropic face sheets and isotropic core $(\mathrm{R}=1)$ or orthotropic core $(\mathrm{R}=0.4$ or 2.5$)$ core [77] 145

Figure 70: $\mathrm{K}_{3}$ for determining maximum core shear stress of a flat rectangular sandwich panel with isotropic face sheets and isotropic core $(\mathrm{R}=1)$ or orthotropic core $(\mathrm{R}=0.4$ or 2.5) $[77]$ 147

Figure 71: Schematic of the graphical optimization with the constraints and feasible set 
Figure 72: The equivalent von Mises stress $(\mathrm{MPa})$ in the composite shell model (for the whole model) 162

Figure 73: The equivalent von Mises stress (MPa) in the layered model (for the first layer: face sheet) 162

Figure 74: The in-plane normal stress, $\sigma_{\mathrm{xx}}=\sigma_{11},(\mathrm{MPa})$ on the core material ................ 164

Figure 75: The in-plane normal stress, $\sigma_{\mathrm{yy}}=\sigma_{22},(\mathrm{MPa})$ on the core material ............... 165

Figure 76: The in-plane shear stress, $\tau_{\mathrm{xy}}=\tau_{12},(\mathrm{MPa})$ on the core material ................... 165

Figure 77: The transverse shear stress, $\tau_{\mathrm{xz}}=\tau_{13},(\mathrm{MPa})$ on the core material ................. 166

Figure 78: The transverse shear stress, $\tau_{\mathrm{yz}}=\tau_{23},(\mathrm{MPa})$ on the core material ................. 166 


\section{Nomenclature}

\begin{tabular}{|c|c|}
\hline \multicolumn{2}{|c|}{ Abbreviation } \\
\hline $\mathrm{AA}$ & Aluminum Association \\
\hline AAR & Association of American Railroads \\
\hline $\mathrm{ACO}$ & Ant Colony Optimization \\
\hline $\mathrm{BC}$ & Boundary Condition \\
\hline CCSA & Constant Criterion Surface Algorithm \\
\hline CEM & Crash Energy Management \\
\hline CES & Cambridge Engineering Selector \\
\hline CFRP & Carbon Fiber Reinforce Polymer \\
\hline $\mathrm{CN}$ & Canadian National \\
\hline CPR & Canadian Pacific Railway \\
\hline DEM & Discrete Element Method \\
\hline EDS & Equivalent Design Space \\
\hline FAA & Federal Aviation Administration \\
\hline $\mathrm{FCC}$ & Face Center Cube \\
\hline $\mathrm{FE}$ & Finite Element \\
\hline FEA & Finite Element Analyses \\
\hline FSW & Friction Stir Welding \\
\hline GA & Genetic Algorithms \\
\hline GHG & Greenhouse Gas \\
\hline GSC & Geometric Stress Concentrations \\
\hline LCA & Life Cycle Assessment \\
\hline
\end{tabular}




$\begin{array}{ll}\text { LRV } & \text { Light Rail Vehicle } \\ \text { MMPDS } & \text { Metallic Materials Properties Development and Standardization } \\ \text { RAC } & \text { Railway Association of Canada } \\ \text { SCC } & \text { Stress Corrosion Cracking } \\ \text { SIMP } & \text { Solid Isotropic Material with Penalization } \\ \text { SPH } & \text { Smooth Particle Hydrodynamics } \\ \text { SQP } & \text { Sequential Quadratic Programming } \\ \text { TLP } & \text { Transient Liquid Phase }\end{array}$

\section{Latin Symbols}

\begin{tabular}{|c|c|}
\hline $\mathrm{P}$ & Lateral force (N/m of length) \\
\hline $\mathrm{W}$ & Weight of lading $\left(\mathrm{N} / \mathrm{m}^{3}\right)$ \\
\hline $\mathrm{h}$ & Depth of lading - not including heap (m) \\
\hline$\varphi$ & Static angle of repose $\left(25^{\circ}\right)$ \\
\hline $\mathrm{P}^{\mathrm{o}}$ & Performance metric \\
\hline $\mathrm{m}$ & Mass $(\mathrm{kg})$ \\
\hline $\mathrm{L}$ & Panel length (mm) \\
\hline $\mathrm{b}$ & Panel width (mm) \\
\hline $\mathrm{t}$ & Thickness (mm) \\
\hline$\rho$ & $\operatorname{Density}\left(\mathrm{Kg} / \mathrm{m}^{3}\right)$ \\
\hline $\mathrm{F}_{\mathrm{cr}}$ & Critical force per unit length $(\mathrm{N} / \mathrm{mm})$ \\
\hline $\mathrm{F}^{*}$ & Desired force per unit length $(\mathrm{N} / \mathrm{mm})$ \\
\hline $\mathrm{M}$ & Bending Moment (M.mm) \\
\hline
\end{tabular}


c

I

$\alpha$

$\sigma_{y}$

$t_{0}$

$t_{f}$

$t_{c}$

$t_{s}$

d

$\mathrm{E}_{\mathrm{o}}$

$\mathrm{E}_{\mathrm{f}}$

$\mathrm{E}_{\mathrm{c}}$

$\mathrm{I}_{\mathrm{o}}$

$\mathrm{I}_{\mathrm{f}}$

$\mathrm{I}_{\mathrm{C}}$

$\mathrm{D}_{\mathrm{o}}$

$\mathrm{D}_{\mathrm{s}}$

$\rho_{\mathrm{o}}$

$\rho_{\mathrm{f}}$

$\rho_{\mathrm{c}}$

$\mathrm{W}_{\mathrm{s}}$

$\mathrm{W}_{\mathrm{o}}$

$\sigma_{\text {yo }}$
Distance from neutral axis $(\mathrm{mm})$

Moment of inertia of the cross section $\left(\mathrm{mm}^{4}\right)$

Load distribution factor

Yield strength of the material (MPa)

Thickness of the normal panel (mm)

Thickness of the facing material layers in the sandwich panel (mm)

Thickness of the core material in the sandwich panel (mm)

Overall thickness of the sandwich panel (mm)

Distance between face sheets mid-planes ( $\mathrm{mm})$

Elastic modulus of the base material in normal panel (GPa)

Elastic modulus of the facing material in the sandwich panel (GPa)

Elastic modulus of the core material in the sandwich panel (GPa)

Moment of inertia of the normal panel $\left(\mathrm{mm}^{4}\right)$

Moment of Inertia of the face sheet in the sandwich panel $\left(\mathrm{mm}^{4}\right)$

Moment of inertia of the core in the sandwich panel $\left(\mathrm{mm}^{4}\right)$

Bending stiffness of the normal panel

Bending stiffness of the sandwich panel

Density of the base material in single panel $\left(\mathrm{Kg} / \mathrm{m}^{3}\right)$

Density of the face sheets in sandwich panel $\left(\mathrm{Kg} / \mathrm{m}^{3}\right)$

Density of the core material in sandwich panel $\left(\mathrm{Kg} / \mathrm{m}^{3}\right)$

Weight of the sandwich panel $(\mathrm{Kg})$

Weight of the single panel $(\mathrm{Kg})$

Yield strength of the base material that the core is mad from (MPa) 


\begin{tabular}{|c|c|}
\hline$\sigma_{\mathrm{y}, \text { core }}$ & Failure strength of the core $(\mathrm{MPa})$ \\
\hline$\rho_{\mathrm{r}}$ & Relative density of the lattice core \\
\hline$[\varepsilon]$ & Shear strain matrix \\
\hline$\gamma$ & Engineering Shear strain \\
\hline$[\sigma]$ & Stress matrix \\
\hline$[\mathrm{C}]$ & Compliance matrix \\
\hline$[\mathrm{A}]$ & Compliance matrix \\
\hline$v$ & Poisson's ratio \\
\hline G & Shear modulus (GPa) \\
\hline $\mathrm{E}_{\mathrm{r}}$ & The relative young's modulus of the lattice core \\
\hline $\mathrm{G}_{\mathrm{r}}$ & The relative shear modulus of the lattice core \\
\hline$\sigma_{\text {ry }}$ & Relative axial yield strength of the lattice core \\
\hline$\sigma_{\mathrm{yl}}$ & Axial yield strength of the lattice core $(\mathrm{MPa})$ \\
\hline$\tau_{\text {ry }}$ & Relative shear yield strength of the lattice core \\
\hline$\tau_{\mathrm{yl}}$ & Shear yield strength of the lattice core $(\mathrm{MPa})$ \\
\hline $\mathrm{P}_{\mathrm{cr}}$ & The Euler critical buckling load $(\mathrm{N})$ \\
\hline $\mathrm{k}$ & Factor that depends on the rotational stiffness \\
\hline A & Cross sectional area $\left(\mathrm{mm}^{2}\right)$ \\
\hline$\sigma_{1}^{\mathrm{cr}}$ & Critical elastic buckling stress of the lattice struts $(\mathrm{MPa})$ \\
\hline$\sigma_{\mathrm{r}}^{\mathrm{cr}}$ & Relative critical buckling strength \\
\hline$\tau_{\mathrm{ij}}$ & Shear stress in $\mathrm{i}$ and $\mathrm{j}$ directions $(\mathrm{MPa})$ \\
\hline$\sigma_{\mathrm{ij}}$ & Normal stress in $\mathrm{i}$ and $\mathrm{j}$ directions $(\mathrm{MPa})$ \\
\hline
\end{tabular}




\begin{tabular}{|c|c|}
\hline $\mathrm{i}, \mathrm{j}$ & Represents $\mathrm{x}, \mathrm{y}$ and $\mathrm{z}$ directions in Cartesian coordinate system \\
\hline $\mathrm{P}_{\mathrm{O}}$ & Uniform lateral pressure (MPa) \\
\hline$\varepsilon_{\mathrm{yc}}$ & The uniaxial yield strain of the core \\
\hline$\varepsilon_{\mathrm{yf}}$ & The uniaxial yield strain of the face sheet \\
\hline$\sigma_{\mathrm{f}, \max }$ & The maximum bending stress on face sheets $(\mathrm{MPa})$ \\
\hline $\mathrm{k}_{2}$ & Coefficient which only depends on the panel aspect ratio \\
\hline$\sigma_{\mathrm{w}}$ & The face sheet wrinkling stress $(\mathrm{MPa})$ \\
\hline $\mathrm{G}_{\mathrm{c}}$ & Shear modules of the lattice core $(\mathrm{GPa})$ \\
\hline$\tau_{c, \max }$ & The maximum core shear stress of the sandwich panel (MPa) \\
\hline $\mathrm{k}_{3}$ & Coefficient dependent upon panel aspect ratio \\
\hline$\delta_{\max }$ & The maximum deflection of the sandwich panel (mm) \\
\hline $\mathrm{k}_{1}$ & Coefficient dependent upon panel aspect ratio \\
\hline $\mathrm{V}$ & Parameter for sandwich panel design \\
\hline$v_{f}$ & Poisson's ratio of the face sheet \\
\hline $\mathrm{a}$ & Panel length (mm) \\
\hline r & Radius of the lattice cell strut cross-section (mm) \\
\hline$v_{l}$ & Poisson's ratio of the lattice core \\
\hline$E_{t}$ & Tangent Modulus (GPa) \\
\hline
\end{tabular}




\section{Chapter 1. Literature Review}

This project is funded by Transport Canada's Clean Rail Academic Grant and is focused on developing new methods and technologies to reduce emissions in Canadian transport sector, especially in the railcar sector. In general, railcars can be divided into two main categories: passenger railcars and freight railcars. There have been several research works about energy saving in passenger railcars and thus, this study is focused on freight railcars.

\subsection{Introduction}

As can be seen in Figures 1-3, although Canada contributes only 1.7\% of global greenhouse gas (GHG) emissions [1], its emissions per capita are among the highest in the world, even higher than top 5 total emitting countries [2].

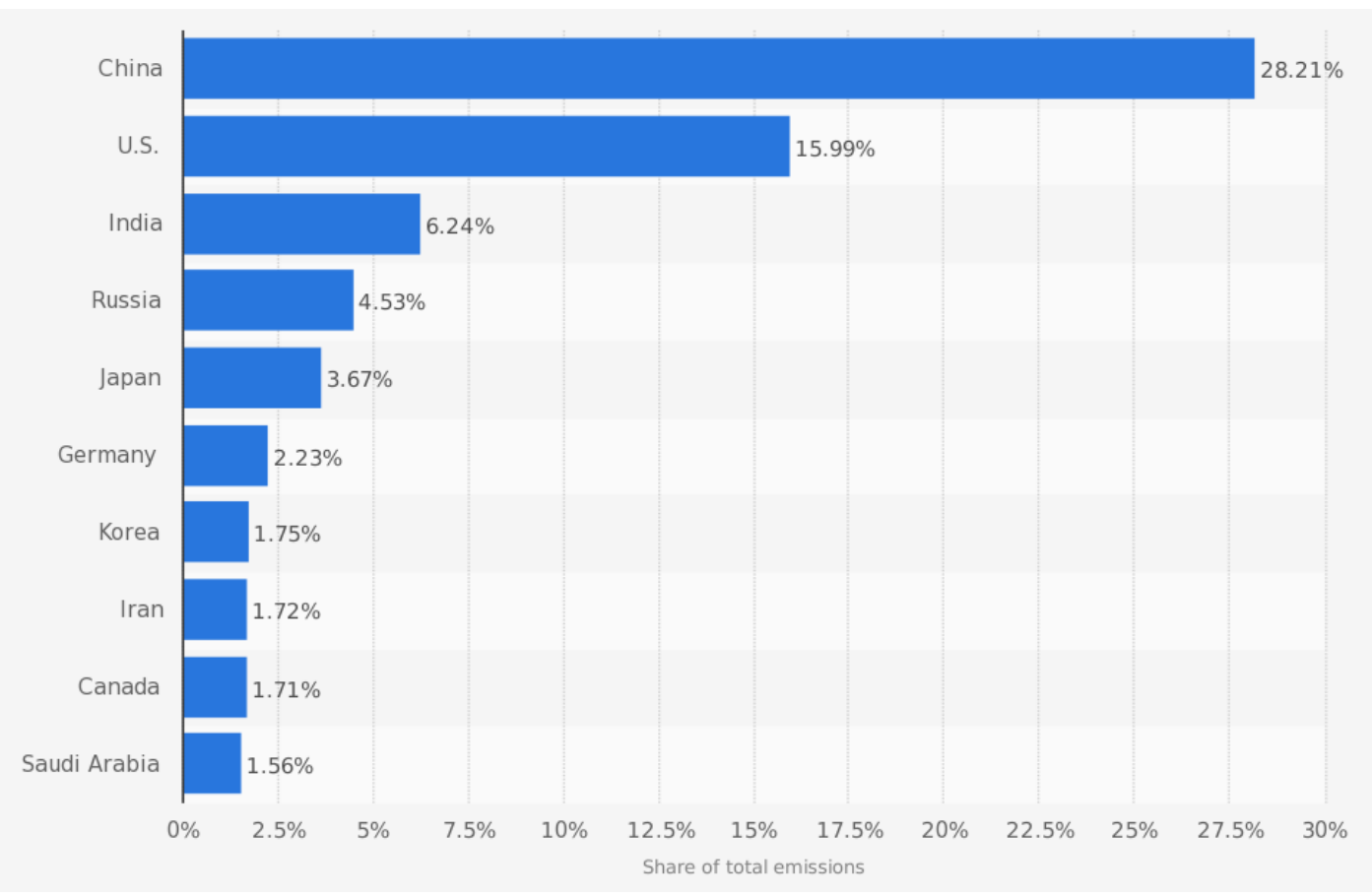

Figure 1: Global share of largest $\mathrm{CO}_{2}$ producing countries in 2016 [1] 


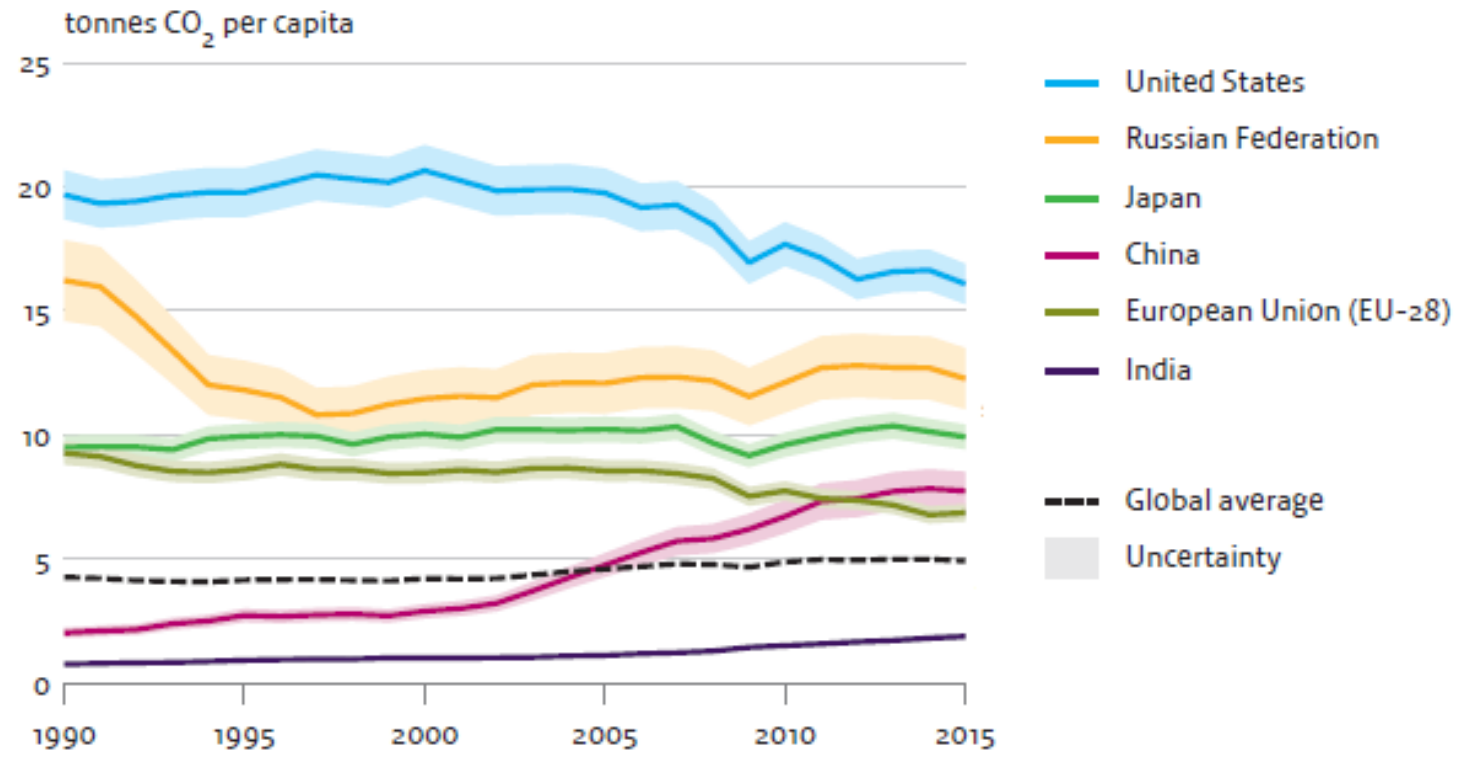

Figure 2: $\mathrm{CO}_{2}$ emissions in the top 5 emitting countries and European Union [2]

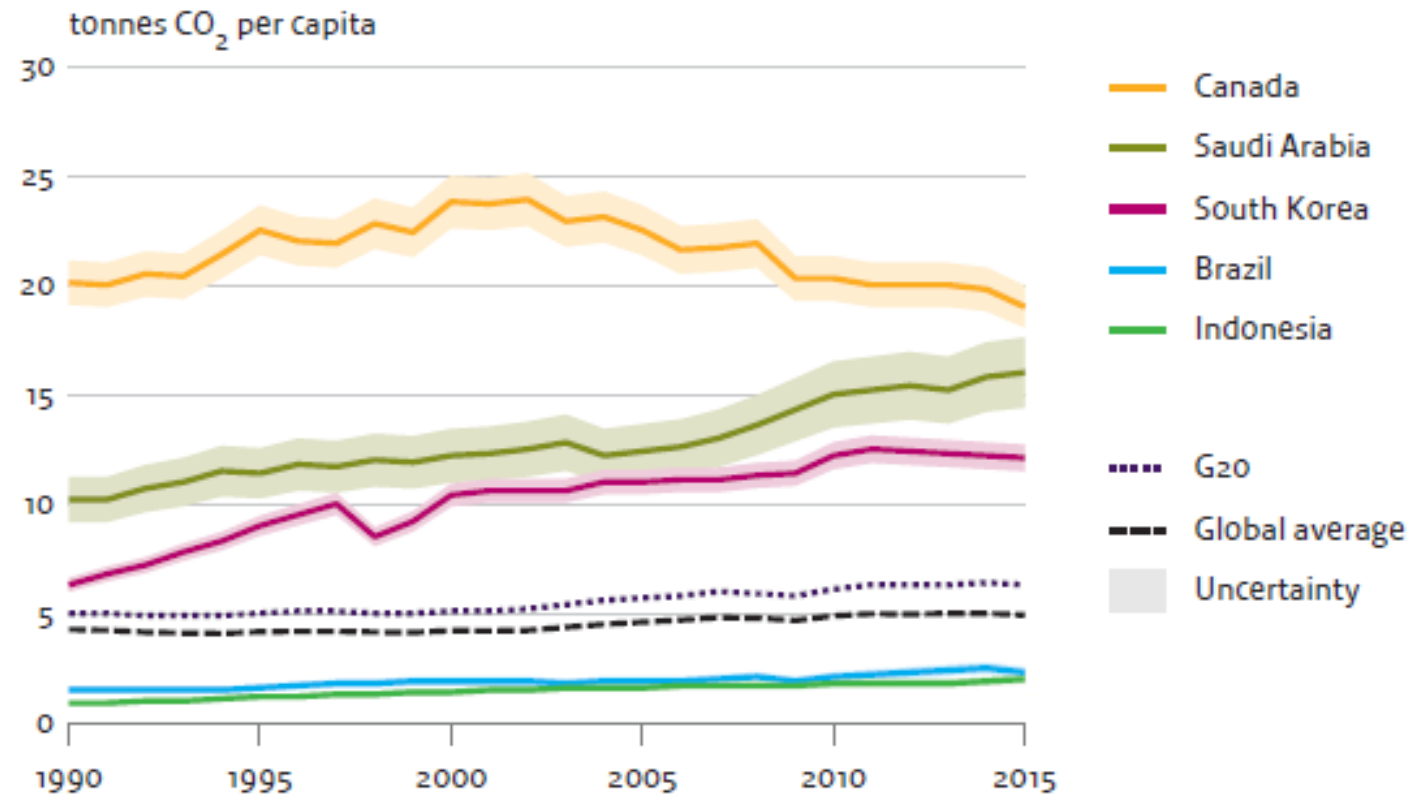

Figure 3: $\mathrm{CO}_{2}$ emission per capita in top 6-10 emitting countries [2] 
Therefore, the Government of Canada developed regulatory measures under Canada's Clean Air Act, to tackle this problem. In fact, the Government of Canada has committed to reduce the nation's total GHG emissions by $17 \%$ below year 2005 levels by 2020 while keeping the economy strong [3]. Figure 4 shows a breakdown of Canada's GHG emission sources from year 1990 to 2015 [4]. The transportation sector has been the second largest contributor to $\mathrm{CO}_{2}$ production since 1990, so it is essential to improve this sector.

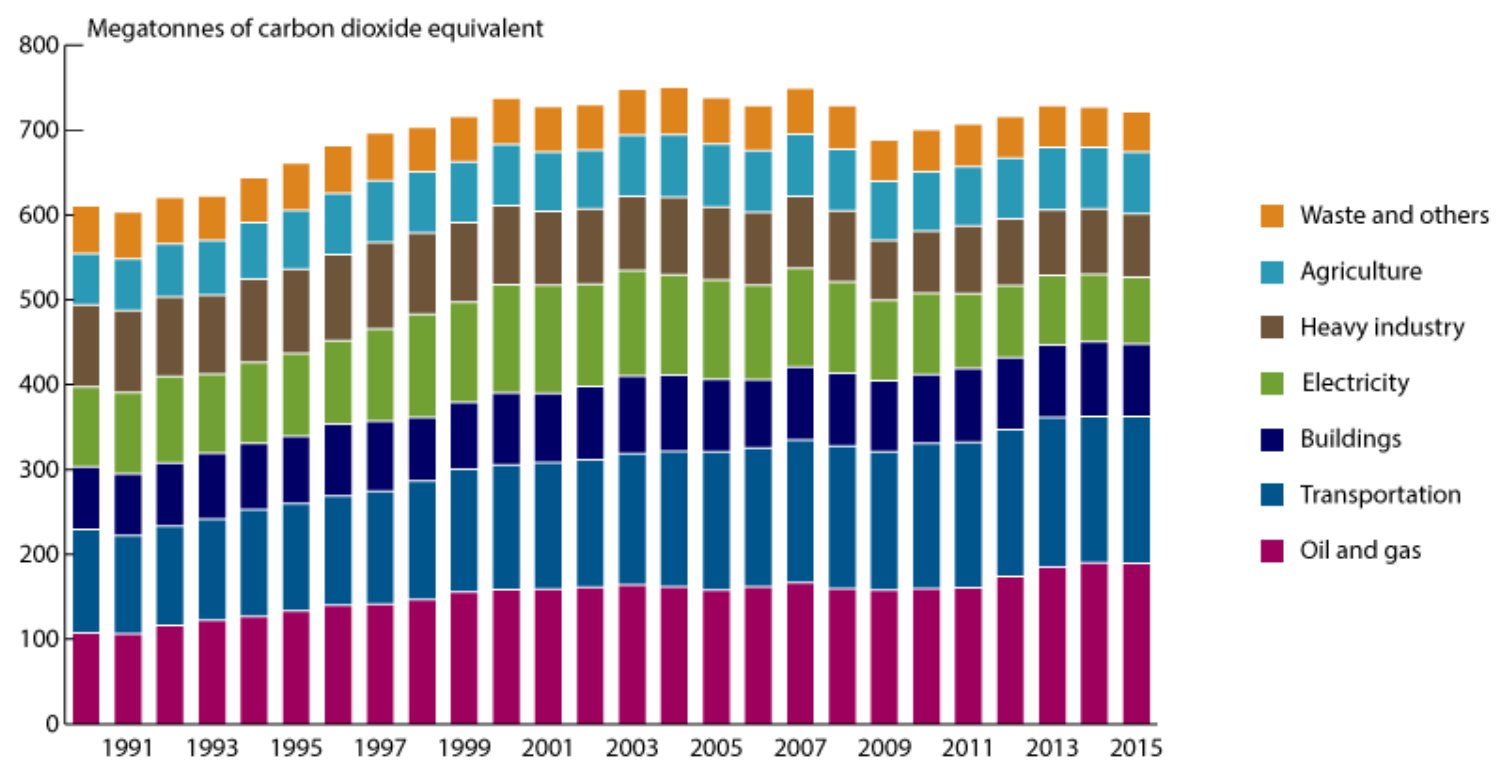

Figure 4: Canada's emission breakdown from 1991 to 2015 [4]

\subsection{Thesis Motivation}

This present research is intended to continue a preceding project, "A Preliminary Study on the use of Fiber Reinforced Polymers in Structural Components" [3], and is focused on developing novel structural concepts for design of lightweight freight railcars, 
which will ultimately reduce emissions in Canadian transport sector. Both the present project and the preceding project have been funded by Transport Canada's Clean Rail Academic Grant (Round \#1 and Round \#3) program. While proposed structural concepts can also be applied to passenger railcars, the focus of this project is on freight railcars.

Freight trains differ significantly in size (number of wagons) and use pattern. The main factors of influence on the energy consumption are the traction type (diesel/ electric), train length and total weight, route characteristics, driving behavior (speed and acceleration) and aerodynamic resistance [5]. As a result, estimating savings in energy consumption or GHG emissions is extremely difficult in the rail sector. For instance, railway operators constantly share freight cars and do not have a fixed number of railcars on each trip [3]. Therefore, instead of investigating the energy saving methods on the whole railcar, only a single freight car wagon will be considered, and by improving the energy consumption of a single wagon, the total energy saving of the railcar will be increased.

There are some reasons to explain why this thesis is not engaged with improving the aerodynamic resistance, while it is one of the most important factors for energy consumption in railcars. First, it highly depends on the length of the train, and in case of studying a single wagon, it will not be as important as it is in studying the energy consumption of the whole train. Second, aerodynamic resistance depends on the speed of the train, and it is stated that the freight cars in North America are on the average known to be slower than in Europe [5]. This also decreases the share of aerodynamic resistance in energy consumption. 
Unfortunately, no studies are currently available on the relative direct energy savings by weight reduction for freight trains. The only reported correlation between energy savings and weight reduction stated that by reducing the weight by $10 \%$, a value of $5 \%$ energy saving will be achieved which is independent of the total train weight, i.e. specific energy consumption. This corresponds to 6.1 tons life-time $\mathrm{CO}_{2}$ savings, assuming $180 \mathrm{~kJ} /$ gross t-km [5]. Both direct and indirect energy savings are the focus of a weight reduction in freight trains. A weight reduction on a railcar with a weight-limited cargo capacity can increase its transport performance or load factor (payload of the railcar as a proportion of the total gross mass). This is referred to as indirect energy saving. In reality, the indirect energy savings will be lower, because freight trains do not always carry the maximum load on each leg. A full load will normally only be achieved on outward bound trips but not necessarily during the return trip. However, on the return trip, direct energy savings will be achieved [5].

\subsection{Weight Reduction Strategies}

As was illustrated, the effect of weight reduction on $\mathrm{CO}_{2}$ emission and energy saving is undeniable. Therefore, the objective of this research is to investigate different weight reduction methods of the structural components on a single freight car. Figure 5 illustrates the top-down method taken by this project to identify the area of interest. 


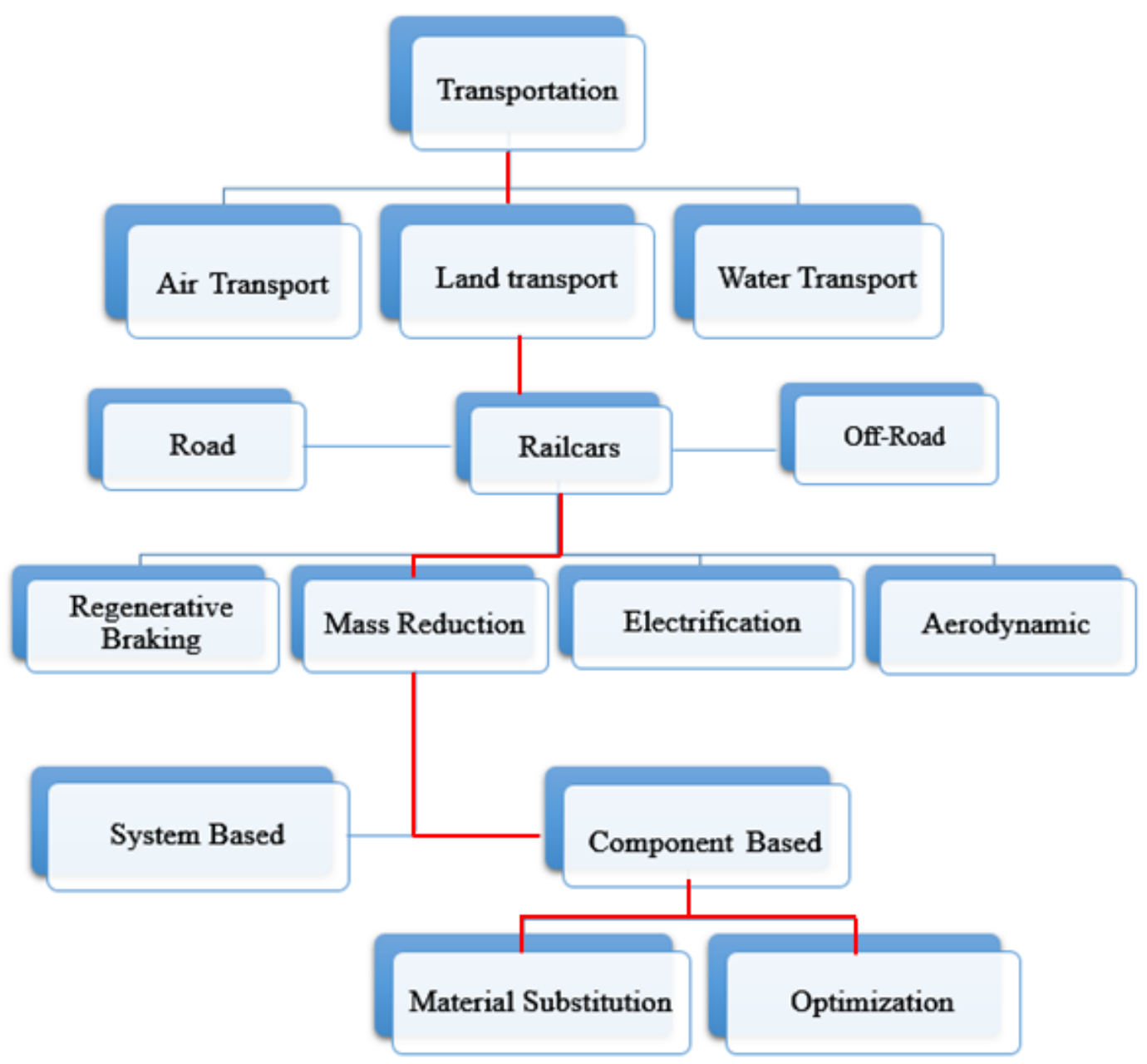

Figure 5: Research path taken to identify areas of interest

There are two different approaches for the above research: material substitution and structural optimization. Although material substitution was done in previous project by replacing the steel with hybrid laminates of fiberglass reinforced epoxy with aluminum face sheets [3], this project is aimed to again review the material selection in more structured way by considering industrial trends, cost, applied loads, etc. and then, optimize the railcar structure with its new selected light weight material according to the design standards of Association of American Railroads (AAR) [6]. In fact, both material 
substitution and optimization will be done simultaneously which will potentially lead to a greater weight reduction. This will be explained in more detail in the following sections.

\subsection{Material Substitution}

So far, aluminum and composites have been used as alternatives for conventional steel railcars. Therefore, a detailed comparison should be made in order to find their weaknesses and advantages. Aluminum has been used in airplanes, passenger railcars, automobiles and even freight railcars for many years and is well known to even nontechnical audience but that is not the case for composites. Thus, first, a general introduction to composite materials will be presented and then, some case studies about comparison of aluminum and composites will be reviewed.

\subsubsection{Introduction to Composite Materials}

Composite materials may provide design engineers with better characteristics such higher strength, lower weight and less maintenance as compared to conventional metals, like steel. Three types of composite materials are developed and widely used in different engineering applications: polymer-matrix composites (PMC), metal-matrix composites (MMC), and ceramic-matrix composites (CMC). According to the reinforcement types, composite materials can be classified into particulate composites, fiber-reinforced composites, and structural composites [7]. These classifications are illustrated in Figure 6 [7]. 

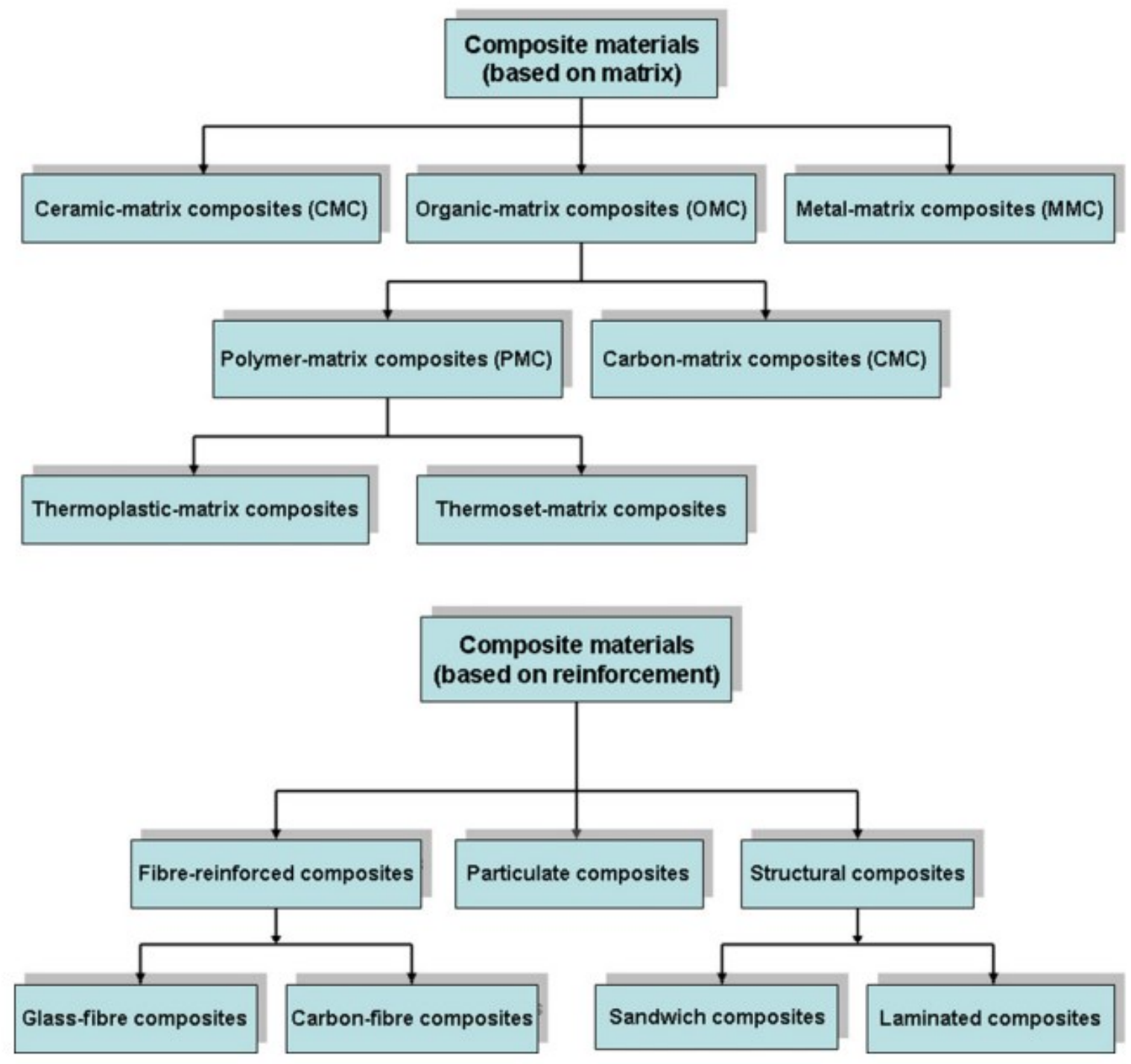

Figure 6: Classification of composite materials [7]

Due to the limited usage of composite materials in the railway industry, usually this industry is categorized under transportation section together with automotive industry or even categorized as "other applications" which makes it hard to find the exact share of rail industry in the world's composite market. However, the application areas of composite materials according to the estimate for the year 2000 is shown in Figure 7. 
Two major application sectors (based on value) are automotive industry (over 30\%) and aerospace industry (over 20\%) [7].

Composites have recently become a primary material for the new generation of commercial aircraft such as the Boeing 787 "Dreamliner" (50\%) and the Airbus A380 (25\%) and A350 (53\%). Weight saving technology in automobiles is crucial for improved fuel efficiency. As the largest application sector, use of composite materials in automotive industry is increasing very rapidly (construction of body, interiors, chassis, hoods and electrical components) [7].

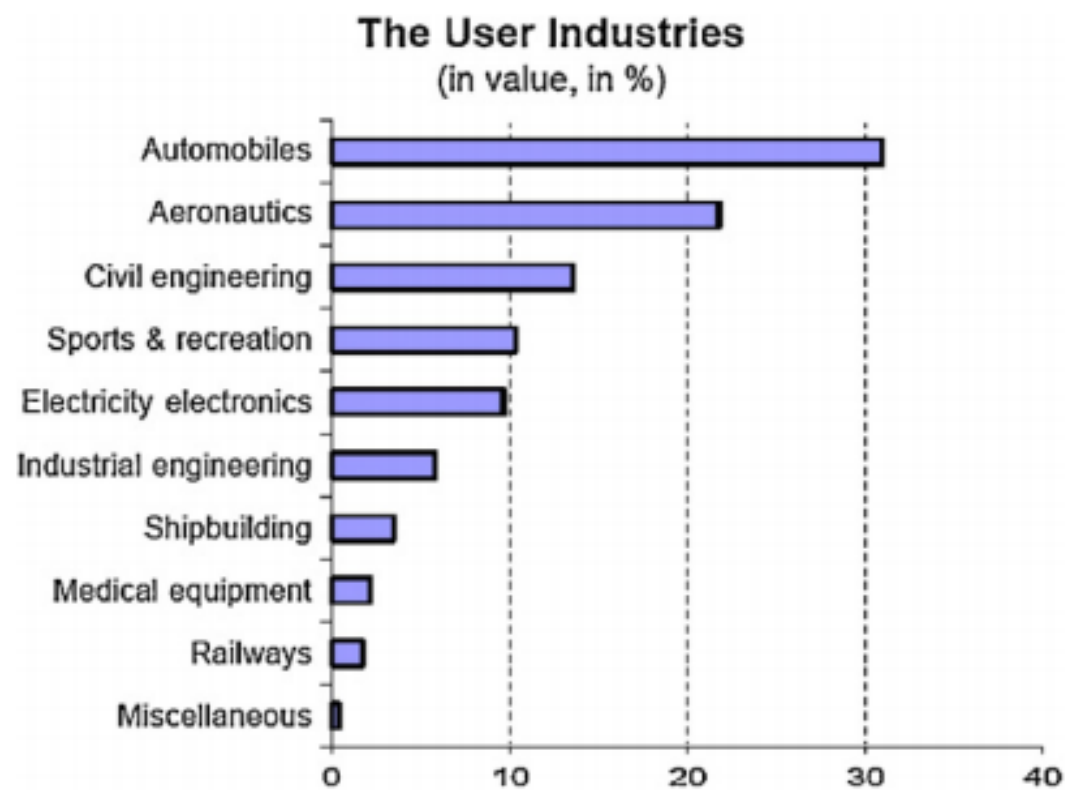

Figure 7: Application of composite materials according to estimates for the year 2000 [7]

The carbon fibre composite material market by application segment for the year 2015 is shown in Figure 8 [8]. As was explained above, although the share of rail industry is not directly mentioned, it can be understood that the rail industry still has a very small portion in the industrial market. 


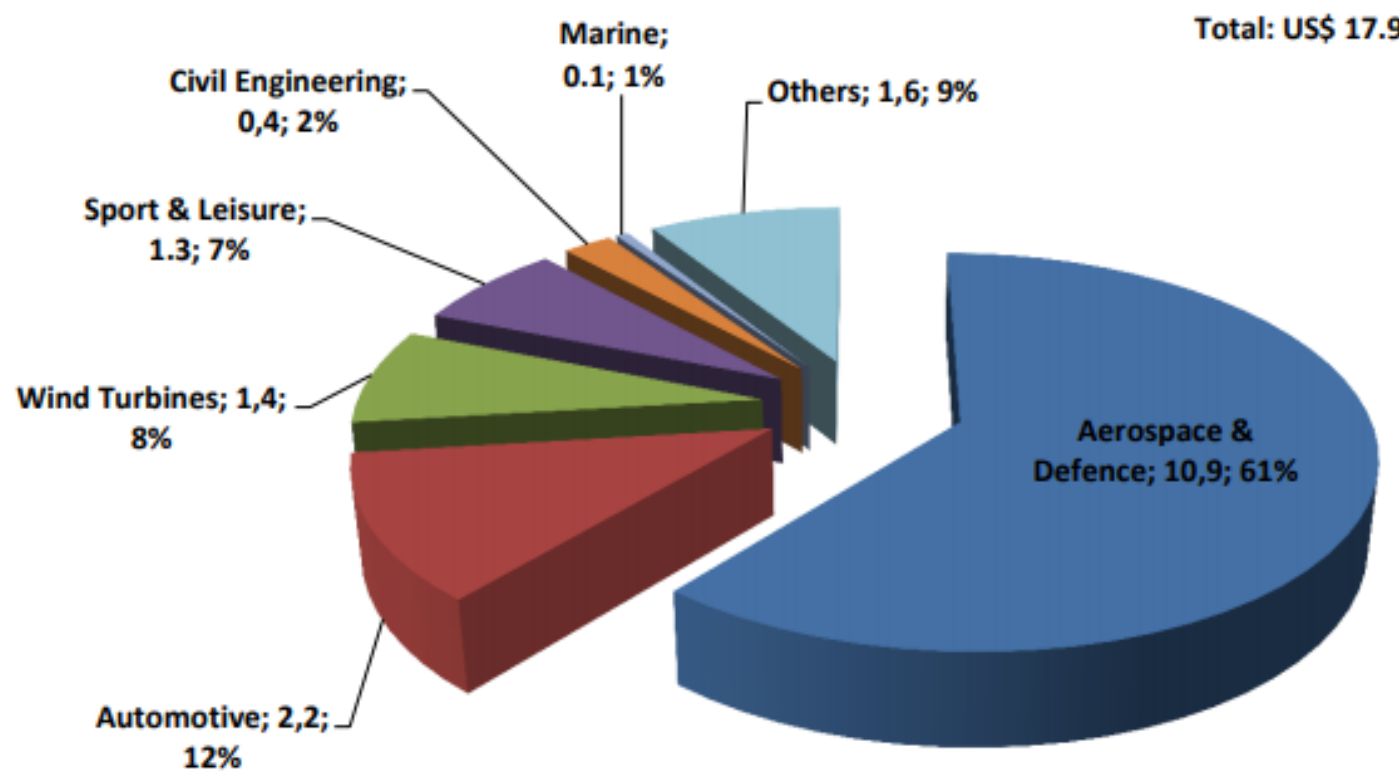

Figure 8: Application of carbon composite materials in the year 2015 [8]

As can be seen in Figures 7 and 8, the application of composite materials in the rail industry is limited. Therefore, its application in other ground vehicles like buses and trucks will also be investigated.

\subsubsection{Prior Case Studies on Lightweight Railcars}

A coupled life costing and life cycle assessment has been performed for car bodies of the Korean Tilting Train eXpress (TTX) project using European and Korean databases, with the objective of assessing environmental and cost performance to aid materials and process selection. Indeed, the research compared different material scenarios and introduced composites as the optimal solution for weight reduction of railcars [9].

Life Cycle Assessment (LCA) is a decision support tool to quantify the effects of goods or services on resource usage and emission burdens. LCA accounts for resource 
consumption and emissions at every stage in a product's life cycle spanning raw material extraction, energy acquisition, manufacturing, use, re-use, recycling, recovery and waste disposal [9]. However, environmentally benign designs and new lightweight technologies will only be widely implemented if the economic interest can be demonstrated. As a result, it is necessary to use another method for cost evaluation which is called Technical Cost Modelling (TCM) [9]. TCM uses engineering, technical and economic characteristics associated with each manufacturing activity to evaluate its cost [9]. This study compares four car-body scenarios [9]:

- Scenario 1: Full composite car-body, with a carbon fiber epoxy aluminum honeycomb sandwich structure and a stainless steel under-frame. This is introduced as an ideal scenario (in terms of low mass) in the Korean Train project.

- Scenario 2: Hybrid composite car-body, with a carbon fiber epoxy aluminum honeycomb sandwich structure, a mild steel inner-frame and a stainless steel under-frame. This is the scenario being explored as an existing alternative to traditional metallic car-bodies.

- Scenario 3: Aluminum car-body, with a 100\% aluminum structure. This scenario is a potential alternative to composite scenarios.

- Scenario 4: Stainless steel car-body, with a $100 \%$ stainless steel structure. This is the current option. 


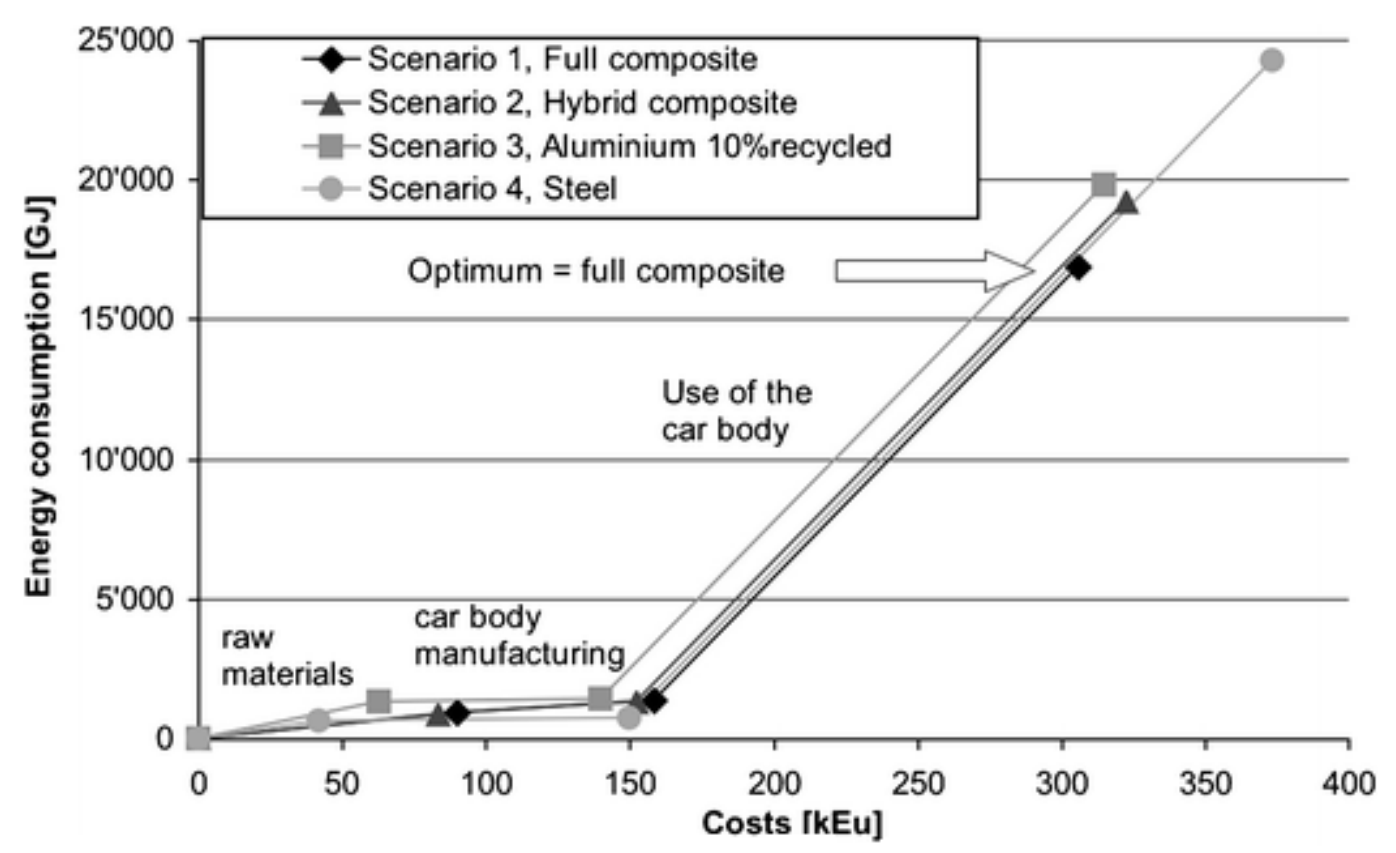

Figure 9: Costs vs Energy consumption [9]

Figure 9 shows that the raw material and manufacturing costs are important $(40-$ $52 \%$ of the total life cycle costs), while the environmental impacts of these phases is low (3-8\%). In the raw material production phase, metallic materials are less expensive to produce but require more energy as compared to composite materials. Steel has the lowest raw material cost but it is also the heaviest system, which increases the energy consumption in use phase, resulting in both the highest life cycle costs and energy consumption. The hybrid scenario is less expensive and has a reduced energy consumption over its whole life cycle, as compared to the steel scenario. It is comparable to the aluminum scenario both in terms of costs and energy consumption. The full composite case gives the optimum solution with the lowest life cycle costs and the smallest environmental impacts [9].

In this specific case study, the use phase clearly had the biggest impact on the environment over the whole life cycle for all four scenarios, with limited contributions 
from the other phases. The recycling of aluminum, however, is important to consider for both energy and costs. To reduce energy consumption in the use phase, the rail carriage weight is a key factor. The composite scenarios therefore represent a credible option for reducing weight and costs. The LCA showed that the full composite scenarios had the lowest impact for all but one midpoint category. Concerning the costs, the full composite car body is the most expensive to manufacture, but the reduction in rail carriage weight enabled large energy savings in the use phase and hence, an overall life cycle cost reduction. This coupled cost and life cycle assessment demonstrates that the full composite variant is clearly the optimum solution [9].

As was mentioned before, the use of composite materials in rail industry is limited. Thus, it is necessary to briefly study their application in other areas which are like railcars, e.g. buses and trucks, to compare the results with the Korean train project.

In another study, the life cycle analysis was conducted to obtain energy saving from lightening vehicle weights [10]. Results are presented in Figures 10 and 11. Figure 10 shows the energy savings throughout the life cycle for trucks when assuming steel parts are replaced with composites or aluminum. The comparison between the steel and composite trucks indicates that a great part of the energy savings is achieved in the use phase and that the composite structure is more environmentally friendly than the steel part. On the other hand, the aluminum truck can save more energy than the composite truck, although aluminum requires more manufacturing energy. The recycling phase makes the biggest contribution to the energy savings of aluminum over composites. The results of the case study for the bus are presented in Figure 11. The overall trend is quite similar to the results of the truck. The longer traveling distance of the bus makes the use 
phase more significant in the life cycle energy savings. In summary, replacing steel with composites in automotive applications results in a positive effect on the energy savings, which is environmentally benign. However, comparing the composite and aluminum vehicles, composite materials turn out to consume more energy over their life time [10].

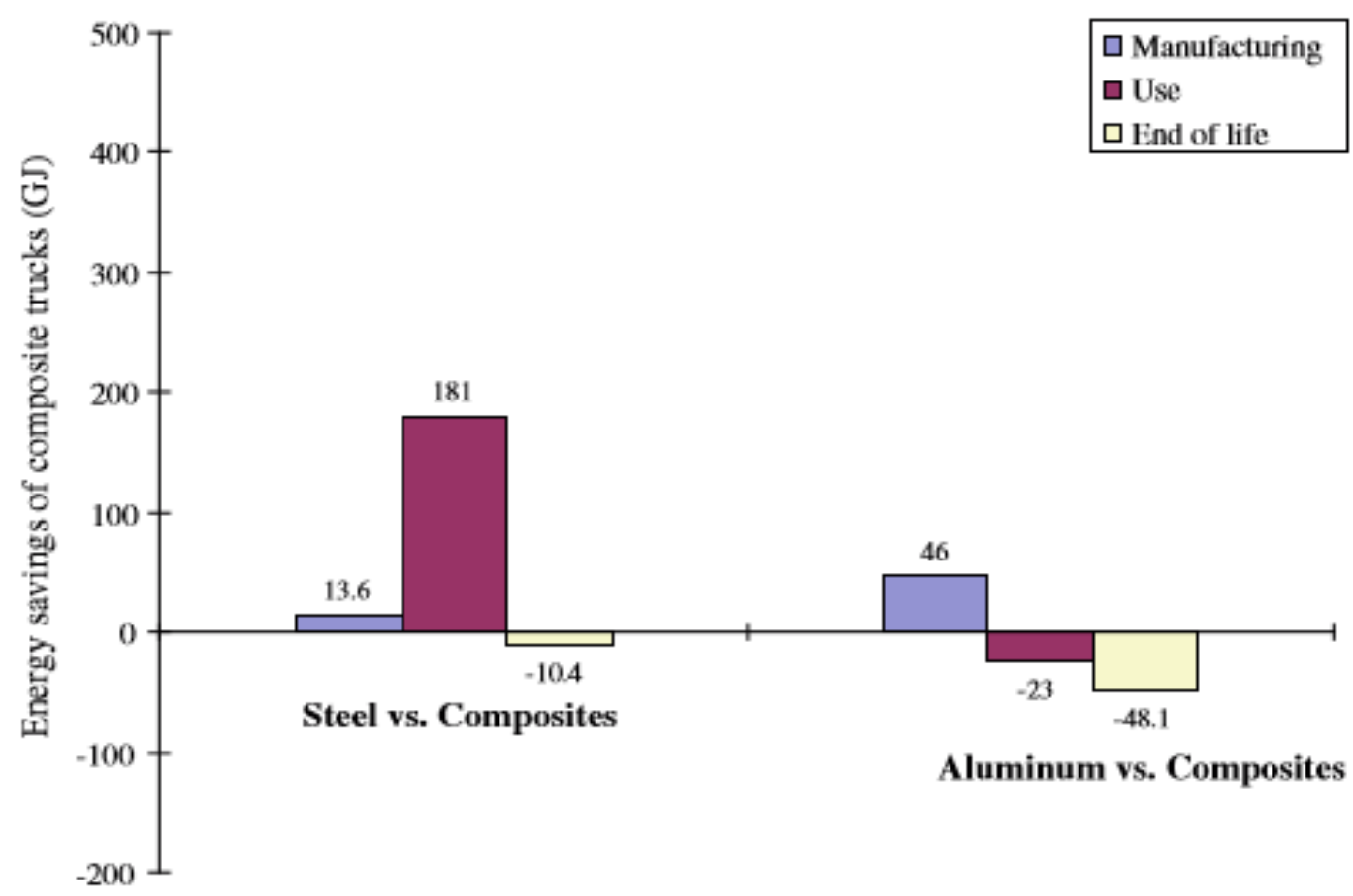

Figure 10: Life cycle energy savings from replacement of steel with aluminum and composites for trucks [10] 


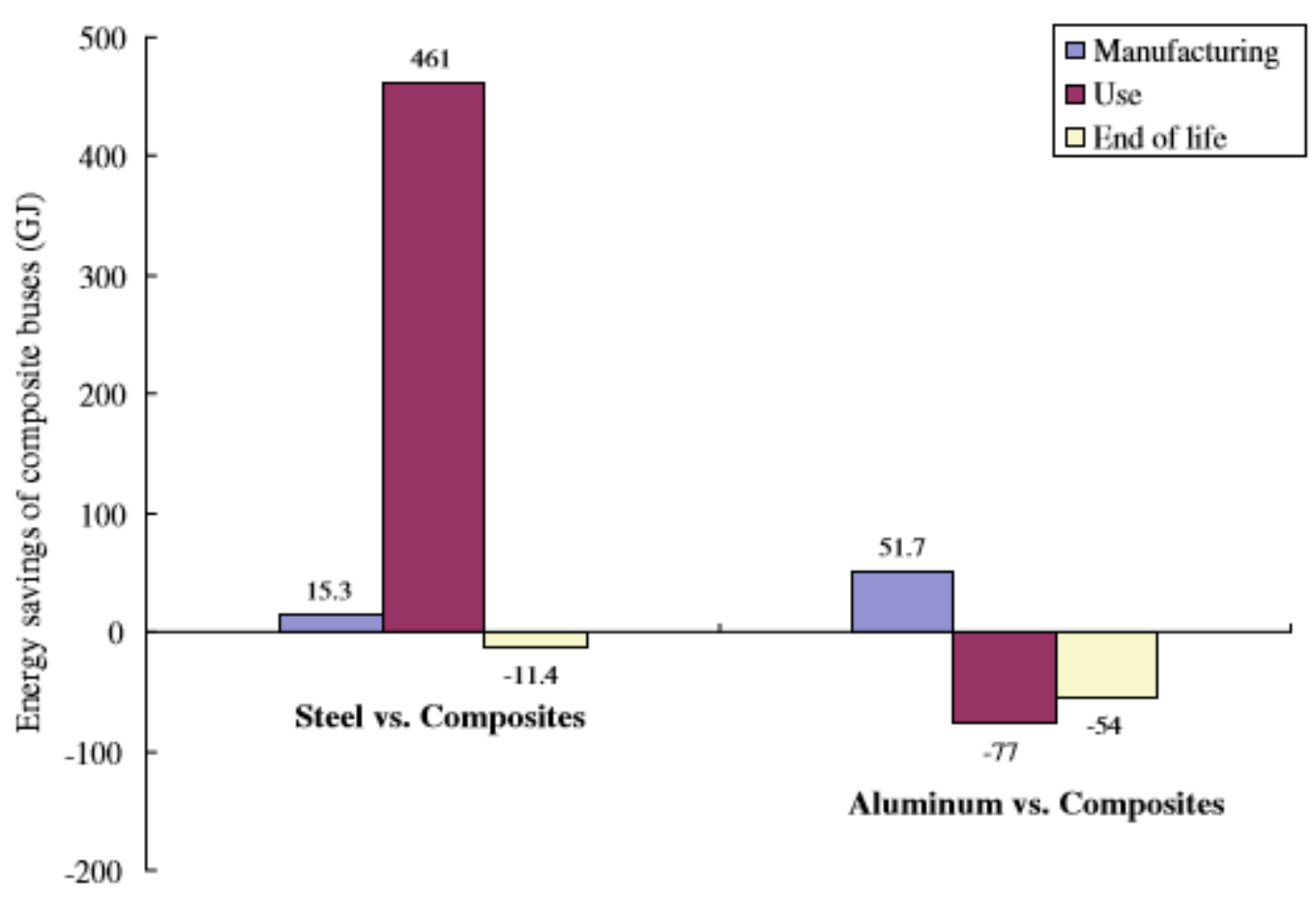

Figure 11: Life cycle energy savings from replacement of steel with aluminum and composites for buses [10]

It was concluded that the composite materials are not always the optimum solution. Different parameters like the type of application, traveling distance and cost must be considered when choosing the alternative light materials for an engineering application. It has also to be mentioned that the end of life phase (disposal or recycling phase) is something that was ignored in the Korean rail project [9] but is a determinant factor when it comes to comparing composite materials with aluminum alloys.

The next step would be the structural optimization of the railcar with its newly selected light weight material. 


\subsection{Optimization}

In this stage, the focus is on structural optimization and weight reduction. The mass removal process can affect different aspects such as structural performance, dynamic behavior, crash worthiness and manufacturability. Therefore, all these aspects must be considered. This stage is started by an introduction to optimization concept and will be followed by considering different design aspects that were mentioned above.

\subsubsection{Introduction to Design Optimization}

Engineering consists of a number of activities, including analysis, design, fabrication, sales, research, and development of systems. The process of designing and fabricating systems has been developed over centuries. The existence of different systems, like automobiles, airplanes and space vehicles well indicates the long history of engineering design. However, the evolution of such systems has been slow and the entire process is time-consuming and costly, requiring substantial human and equipment resources. In fact, some systems can usually accomplish the same task better than others. For example, the purpose of a bridge is to provide continuity in traffic from one side of the river to the other side. Several types of bridges can serve this purpose [11]. This is the stage where the optimization comes to the design process. In fact, the goal of optimization is to find the best solution among all possibilities, so the product would be more efficient and cost effective. The purpose of finding the best solution is not limited to a specific field and can be applied to the wide variety of areas like structural design or control and dynamic systems [11]. Nevertheless, the focus of this research is on structural optimization. 
Structural optimization is the subject of making an assemblage of materials that sustain loads in the best way. The term "best" must be defined based on the type of problem. It could be to make the structure as light as possible, as stiff as possible or even as resistant to buckling or instability as possible [12]. In general, structural performance factors mainly used as both constraints or objective functions are weight, stiffness, critical load, stress, displacement and geometry [12]. However, in structural optimization, usually geometry parameters are used as design variables to maximize or minimize other mentioned structural performance parameters. Depending on the geometric feature, structural optimization problems are divided into three classes: Sizing optimization, Sizing optimization and Topology optimization [12].

Sizing optimization: This is when the design variable is some type of structural thickness, i.e. cross-sectional areas of truss members or the thickness distribution of a sheet. A sizing optimization problem for a truss structure is shown in Figure 12 [12].

\section{Initial design}

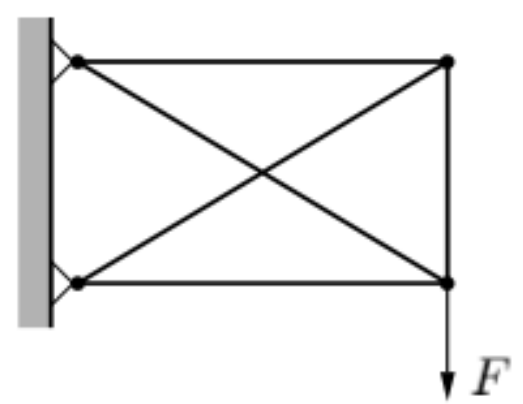

Optimized design

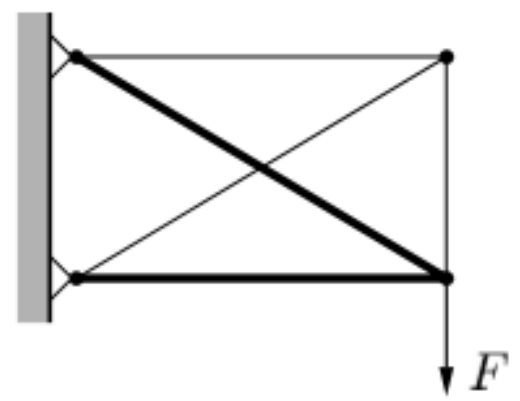

Figure 12: A sizing structural optimization problem [12] 
Shape optimization: In this case, the design variable represents the form or contour of some part of the boundary of the structural domain. Consider a solid body which is described by a set of partial differential equations. The optimization consists in choosing the integration domain for the differential equations in an optimal way. A twodimensional shape optimization problem is seen in Figure 13 [12].

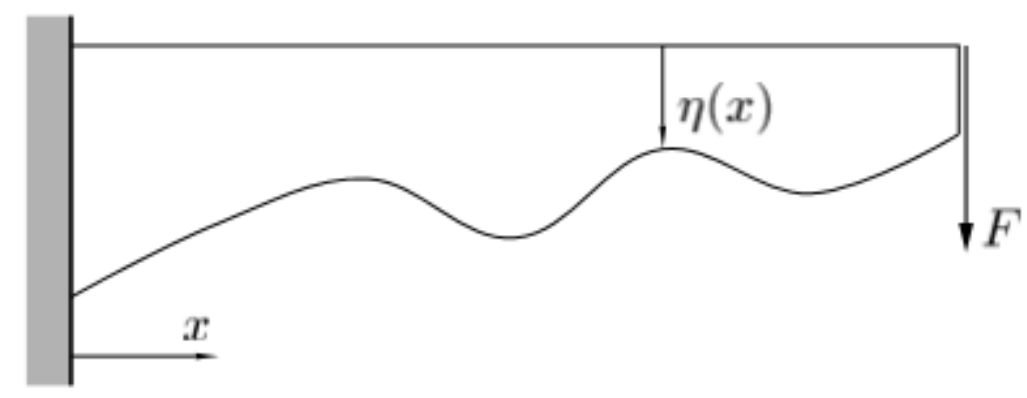

Figure 13: A shape optimization problem [12]

Topology optimization: This is the most general form of structural optimization. In a discrete case, e.g. for a truss, it is achieved by taking cross-sectional areas of truss members as design variables, and then allowing these variables to take the value zero, i.e. bars are removed from the truss. This can be seen in Figure 14(a). If instead of a discrete structure a continuum-type structure such as a two-dimensional plate is considered, then a new topology can be achieved by letting the thickness of the sheet take the value zero. In a three-dimensional case the same effect can be achieved by letting the design variable be a density-like variable that can only take the values 0 and 1 . An example of this kind of topology optimization is shown in Figure 14(b) [12]. 


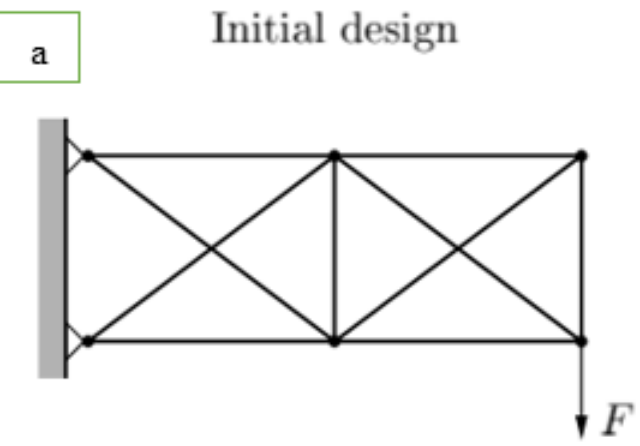

\section{Optimized design}
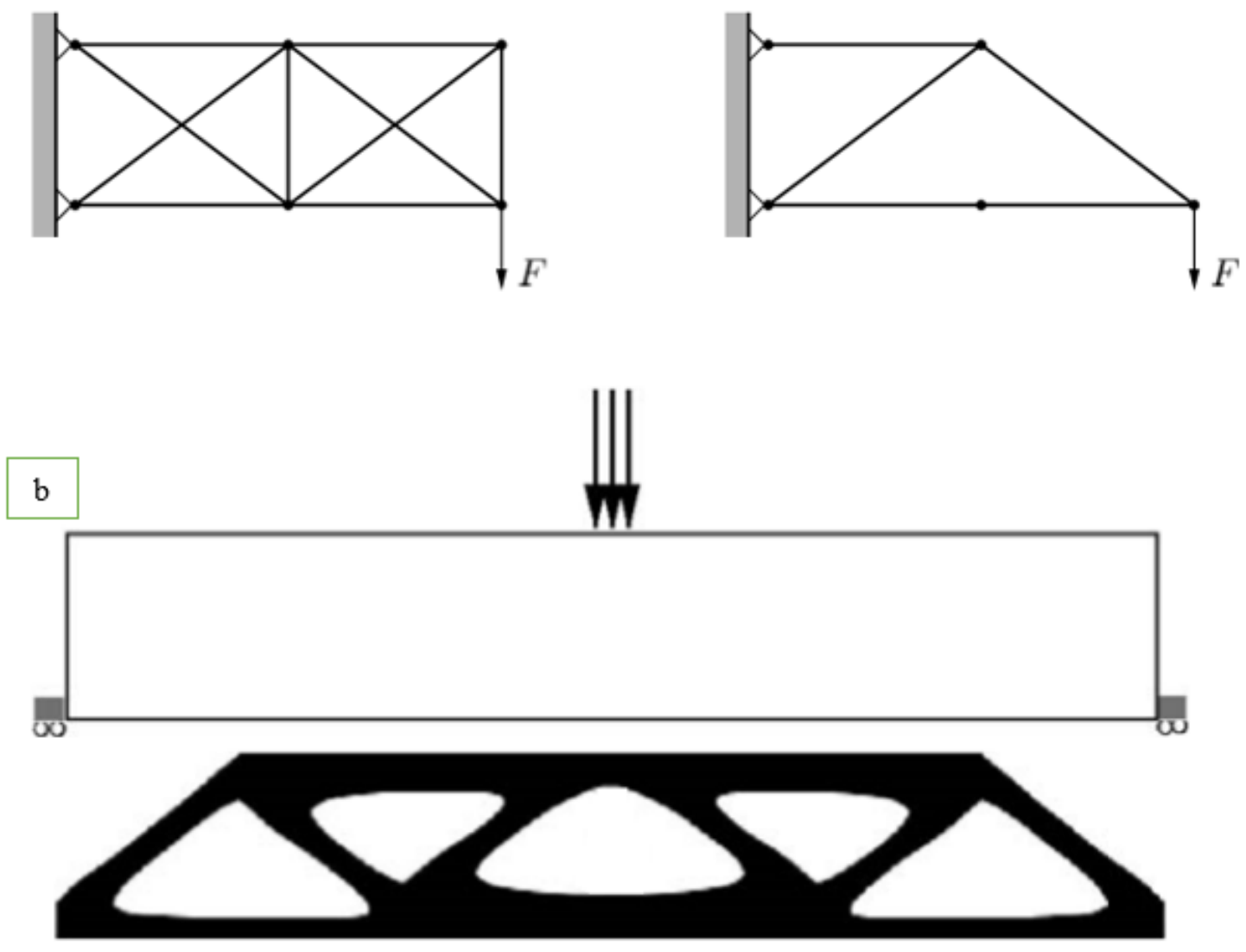

Figure 14: Topology optimization of (a) discrete system, and (b) continuous system [12]

\subsubsection{Dynamic Behavior and Crashworthiness}

It is known that the weight reduction may affect dynamic behavior and crashworthiness of rail vehicles. Therefore, in addition to structural characteristics, these features should be considered as well.

Using light materials and altering mechanical structures can often result in deterioration of rigidity of the car body. This reduction in the rigidity of a car body is one of the primary causes of low modal frequencies. The vibration of lightweight car bodies 
with low modal frequencies may occur in a high-sensitivity range for passengers, leading to poor ride comfort for railway passengers [13]. Thus, quantitative regulations regarding the Eigen-frequencies of railway vehicle car bodies have been reported and many countries have specific frequency requirements [13]. However, the focus of this thesis is not on passenger rail cars, and thus, lower natural frequencies are not an issue unless some restrictive regulations has been set by the AAR [6] on the minimum allowable natural frequency in freight railcars.

It has also shown that improving the dynamic characteristics of the suspension system can increase the natural frequency of an optimized car body into an acceptable range without any further changes to the structural properties [13]. Weight reduction can also lead to dynamic instability of railcars, especially in high speed trains and curved paths [13] but since these types of railcars have relatively low speeds, dynamic stability problem will not be crucial. To sum up, it must be mentioned that dynamic behavior of light weight vehicles is currently an active research field but as is involved with pure dynamic analyses, it would be out of the scope of this thesis.

Weight reduction can also result in lower levels of crashworthiness in road and rail vehicles. Crashworthiness can be defined as the ability of a vehicle to protect passengers and cargo during the impact. There are some studies in this area with the aim of improving the crashworthiness of passenger railcars. For example, effectiveness of alternative rail passenger equipment crashworthiness strategies was investigated by Jacobsen et al., 2006 [14]. In his work, crashworthiness strategies including crash energy management (CEM), pushback couplers and push/pull operation were evaluated and compared for five different cases. Crash energy management (CEM) is a design 
technique that enhances crashworthiness. CEM seeks to control the load path into the car body structure in a way to absorb the energy with components that are outside the occupied volume [14]. The five different cases are [14]:

1- All conventional cars with a cab car leading (baseline case).

2- All conventional cars with a locomotive leading.

3- Conventional cars with pushback couplers, with CEM cab car leading.

4- All CEM cars with a cab car leading.

5- All CEM cars with a locomotive leading.

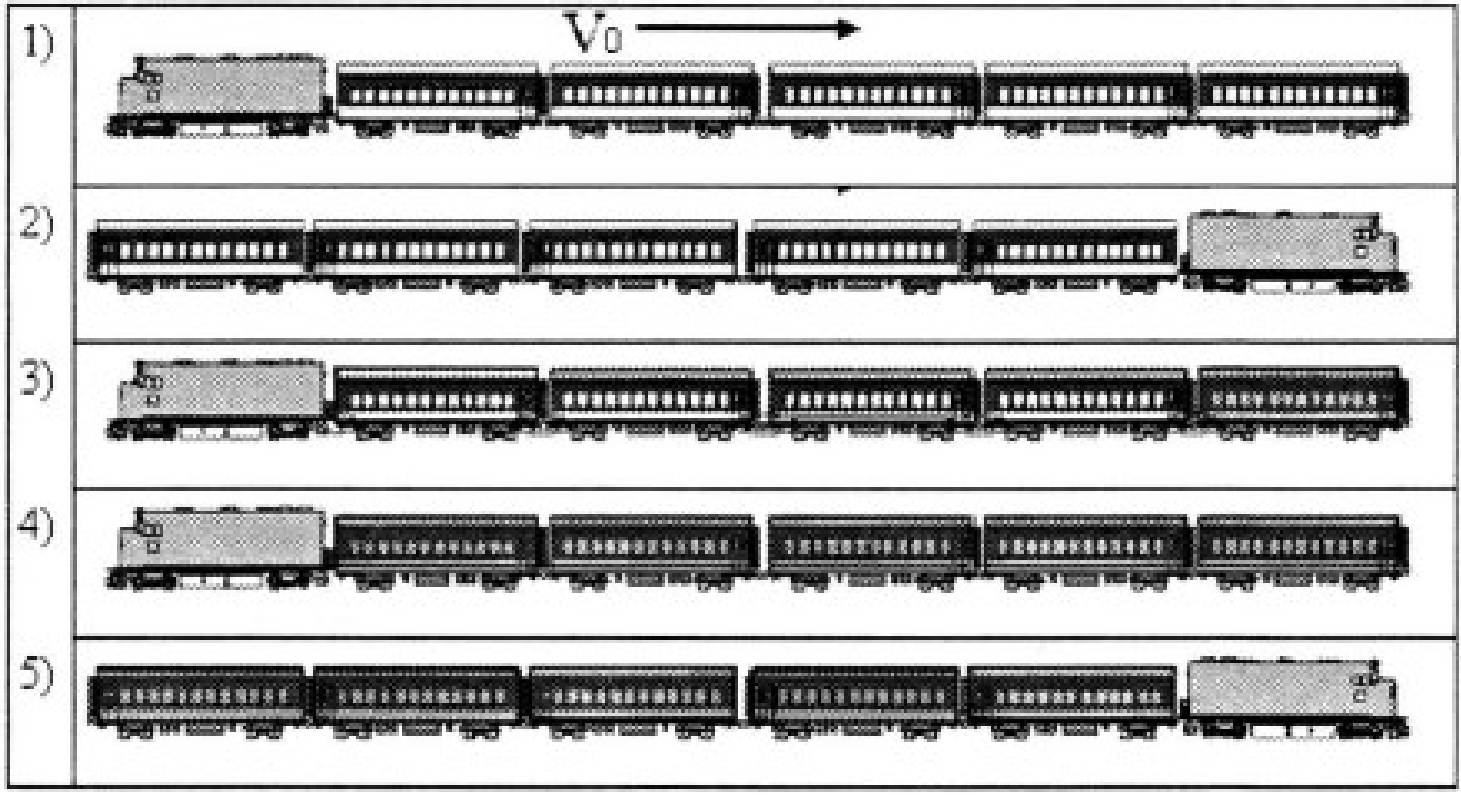

Figure 15: Collision cases in moving train [14]

The impact velocity was ranged from 10 to $40 \mathrm{mph}$ and a one-dimensional lumped-mass collision dynamics model was used to evaluate the effectiveness of each strategy, or combination of strategies, in terms of preserving survivable space for 
occupants. It was shown that of the five cases evaluated, the scenario 1 (a cab car led conventional cars) represents the baseline level of crashworthiness. The highest levels of crashworthiness are achieved by the scenario 5 (a consist of all CEM cars with a locomotive leading), followed by the scenario 4 (all CEM cars with a cab car leading). The results indicate that improvements in collision safety can be made by applying different combinations of these crashworthiness strategies. A CEM cab car leading conventional cars (scenario 3) that are modified with pushback couplers enhances the level of crashworthiness over a conventional cab car led and provides a level of crashworthiness equal to a locomotive leading conventional passenger cars (scenario 2) [14].

Although Jacobsen's study was focused on improving crash behavior, he did not consider structural optimization and possible effects of mass reduction. However, in the following section, some of previous researches in the field of structural optimization and minimizing its undesirable effects will be discussed.

\subsection{Previous Studies on Light Weight Design of Railcars}

Multifunctional optimization of a composite high-speed train structure was studied by Wennberg and Stichel [15]. Their design methodology for a train car body is illustrated in Figure 16 [15]. Level A is choice of design space, for a rail vehicle this would be limited from the outside by the track profile and from the inside by the desired amount of space available for passengers. Level B is the topology and size optimization of the structure based on safety requirements, e.g. strength and stiffness. Level $\mathrm{C}$ is a global finite element (FE) and the load case evaluation. Level D is a sub-level to level C 
and consists of local evaluation of a characteristic section based on the global load cases of level C. The FE model of level D can either be a completely separate model or simply a part of the global model from level C. Stress analysis is performed on this level. Level E consists of the multifunctional optimization. If the constraints of the 1D optimization in Level $\mathrm{E}$ are altered to, for example, reduce the strength and/or stiffness of the global model, the evaluation in level $\mathrm{C}$ (or possibly level $\mathrm{D}$ ) examines if the reduction in strength and/or stiffness on the local level is within safety requirements for the global level. If not, the C-D-E loop can be re-run with altered constraints [15].

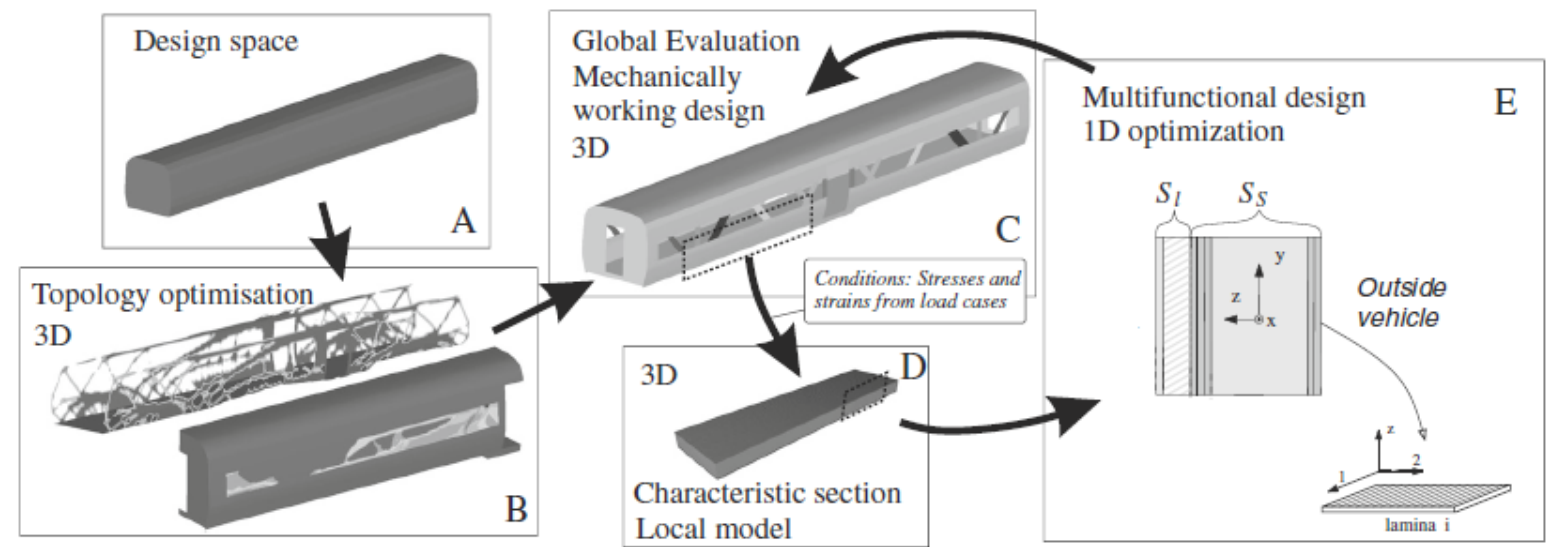

Figure 16: Overview of design methodology [15]

The method consisted of five different levels, A-E, but their study was focused on level C-E. The structure used in level $\mathrm{C}$ was derived from one of previous research works of the same authors, Wennberg et al., 2012 [16]. The selected characteristic panel consists of several layers which can be grouped in the load carrying structure $\left(S_{S}\right)$, which is a three-layer sandwich panel consisting of carbon fiber face sheets and a PMI (Polymethylacrylimide) core, and the two-layer structure $\left(S_{l}\right)$ comprising an inner-lining 
(glass fiber/vinyl ester) and a layer of fibrous insulation. Besides the commonly used design constraints, such as mechanical strength, stiffness and geometry, also acoustic and thermal insulation as well as fire safety was included in their optimization in level E. This was done by using Sequential Quadratic Programming (SQL) method. The final structure has $17 \%$ mass reduction as compared to the starting structure [15]. This was a good result since the starting structure was an optimized load carrying sandwich panel which by itself reduced the weight of a comparable steel structure by $40 \%[15,16]$. The structure derived also fulfils thermal, fire and acoustic constraints at this low weight. This result came at a cost of reduced Eigen frequencies of the car body [15].

Kuczek investigated the application of manufacturing constraints on structural optimization of thin-walled structures, particularly in rail vehicles [17]. It is known that the topologically optimized structures often turn out to be difficult to manufacture without specialized methods such as additive manufacturing. Thus, the optimized design should be made of standardized, commercially available profiles. For this reason, manufacturing constraints must be considered in the optimization procedure. Typical manufacturing constraints described in the literature include casting, extrusion and stamping. However, a new design space preprocessing methodology called Equivalent Design Space (EDS) was presented in his work. For that, the element stiffness of a solid cube reduced to the stiffness of a thin-walled cube of similar dimensions [17]. The stiffness of a thin-walled cube was found by application $100 \mathrm{~N}$ force and thereby obtaining the value of its linear deformation. Dimensions of the cube were selected based on the cross-sectional dimensions of a standard profile to be used for the railcar design, i.e. $180 \times 180 \times 180 \times 5 \mathrm{~mm}$. The stiffness of a solid cube was found in the same way by 
applying a force of $100 \mathrm{~N}$, thereby obtaining the value of the linear deformation. The linear deformation of the solid cube turned out to be smaller than that of the thin walled cube. Therefore, the stiffness of this model was tuned to reach the value of linear deformation equal to the first model. The stiffness of the solid model after stiffness tuning was used for topology optimization. This was done because the manufacturing requirements assume making railcar design with thin-walled profiles [17].

As an example of Kuczek's optimization methodology, a freight railcar structure was selected. The optimization problem was to find the minimum mass of a freight structure subjected to static von Mises stress, geometrical and manufacturing constraints. Solid Isotropic Material with Penalization (SIMP) method was used for the optimization. In the SIMP method, a pseudo material density is the design variable. The material density varies continuously between zero and one, with zero representing the void state and one the solid state. The topology optimization stopped when all constraints were satisfied, and ultimately, an easily manufactured structure was obtained [17].

Harte et al. used a multi-level approach in the optimization of the body structure of a Light Rail Vehicle (LRV) according to industrial standards [18]. Their selected body shell had a sandwich panel configuration and was supported on a steel stiffener frame. The sandwich panel comprised face sheets made from glass fiber reinforced epoxy composite plies with a polyurethane foam core. Their work was aimed to optimize the body shell wall thickness and use shape optimization to reduce possible stress concentration at window openings. A multi-level approach was used whereby the total structure was first analyzed using effective composite properties to identify the most highly stressed panel. This panel was further divided into subdomains within which the 
body shell wall parameters such as ply thickness and shape geometrics were defined for optimization [18].

Hudson et al. described the application of Ant Colony Optimization (ACO) algorithm to the multiple objective optimization of both low mass and low cost for a metro rail vehicle floor sandwich panel [19]. Despite the fact that most other material selection studies were limited to few material options, their aim was to keep the range of material combinations large to allow any potentially new or less obvious solutions to be discovered. For the face materials, the optimization algorithm was provided with a range of options to choose from including various aluminums, steels, fiber-reinforced polymers and wood products. Furthermore, for the fiber-reinforced polymer facings, the algorithm could select between a range of fiber and matrix materials, as well as specifying the fiber volume fraction, the number of plies in the laminate, and the orientation angle of each ply $\left(0^{\circ}, \pm 45^{\circ}\right.$ or $\left.90^{\circ}\right)$. Similarly, a number of core material options were available, including a variety of polymer foams, honeycombs and balsa woods of different densities. In total, there were 40 different core and facing materials for the algorithm to choose from. This material database, when coupled with the fiber-reinforced polymer laminate design options, provided a very large number of potential sandwich material combinations. However, as in most of multiple objective optimization problems, there was no single optimal solution in their work. Instead, a series of solutions introduced that each contained an element of optimality [19].

In other research, Mrzyglod and Kuczek studied a uniform crashworthiness concept optimization for high-speed passenger train car bodies [20]. It was shown that it is possible to improve the passive safety without increasing vehicle mass by using 
modern methods and the tools for structural optimization. The design optimization was done from the point of view of structural protection of occupants' survival space. The optimization strategy consisted of three stages. In the first step, the constant criterion surface algorithm (CCSA) of topology optimization was applied to find a preliminary solution. To improve the manufacture properties of this solution, a set of design space constraints was proposed. In the second stage, the parametric model of the structure was used. The parametric model was built taking into consideration the results of the first stage optimization. The first stage finite element model of solid type elements was replaced by an equivalent FE model of shell type elements. The shell thickness of the FE model was parameterized. This model was optimized using genetic algorithms (GA). The solution obtained from the GA optimization was set to be a starting point for the third stage of optimization. Finally, CCSA optimization was applied again to remove excessive material from the thin-walled FE model [20].

Liu and Wakeland investigated a new optimization procedure on the bottom section of a boxcar body end structure consisting of an end sheet reinforced by two channels, see Figure 17 [21].

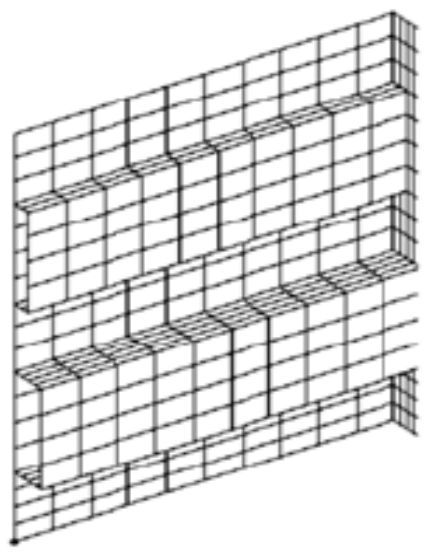

Figure 17: FE model of the boxcar end structure [21] 
They introduced a method to combine a sizing optimizer with metamodeling for topology optimization called a Hierarchical, Interactive, and Metamodel-based Optimization (HIMO) [21]. The summary of what they did is outlined below [21]:

- Step 1. Sampling points were obtained by using statistical methods for experimental design, each point represents different configurations or layout options of the structural systems.

- Step 2. Finite element analyses (FEA) run for every sample design to identify the feasible designs in terms of major design constraints such as strength, deflection, as well as bound limits for sizing variables. At the same time or later, size optimization can be performed to find the optimum thickness designs. Each resulting feasible and optimal design corresponds to one of the layout design determined in step 1 .

- Step 3. A metamodel representing the objective function and design constraints, i.e. the weight versus the topology design variables, was then built to fit the optimal solutions derived in step 2.

- Step 4. By using Sequential Quadratic Programming (SQP), the upper level optimization was done to find the best point on the response surface.

- Step 5. Finally, an FEA run again on the optimal solution obtained from step 4 to find the final result which is optimal in terms of both sizing and topology. By reviewing previous studies on lightweight design of railcars, it can be observed that most of the research was focused on passenger railcars, especially for highspeed trains, and the new designs were implemented according to European design and safety standards. Very few studies have investigated the application of lightweight design 
techniques in freight railcars, and the situation is even worse when it comes to design of these cars according to North American rail standards. In fact, a lack of research works in structural design of freight railcars according to North American standards is obvious, and this is the area that the current thesis is aimed to investigate.

\subsection{Review of The Canadian Rail System Operation}

The Railway Association of Canada (RAC) recognizes three Canadian Class I railway members: Canadian National (CN), Canadian Pacific Railway (CPR or CP), and VIA Rail. Combined, these members consume approximately $94 \%$ of all locomotive

diesel fuel burned in Canada. The $\mathrm{CP}$ and $\mathrm{CN}$ are freight railcar operators who ship products (such as grains, oil, other bulk commodities and intermodal containers) across Canada and the USA, whereas VIA Rail is the primary intercity passenger rail operator within Canada. However, as was mentioned, the focus of this research is on freight railcars. Hence, only the two freight railcar operators, $\mathrm{CP}$ and $\mathrm{CN}$, and their relevant information will be considered [3].

The AAR [6] has designated special car classification codes for each freight car type to be used in a computerized traffic-generated program. That is, these classifications are used to track the prototypical operation of each classification to be categorized in terms of weekly railroad performance measures [3]. The car types and their code, relevant to this research are listed in Table 1 [3]. 
Table 1: List of some AAR freight car types and their corresponding codes [3]

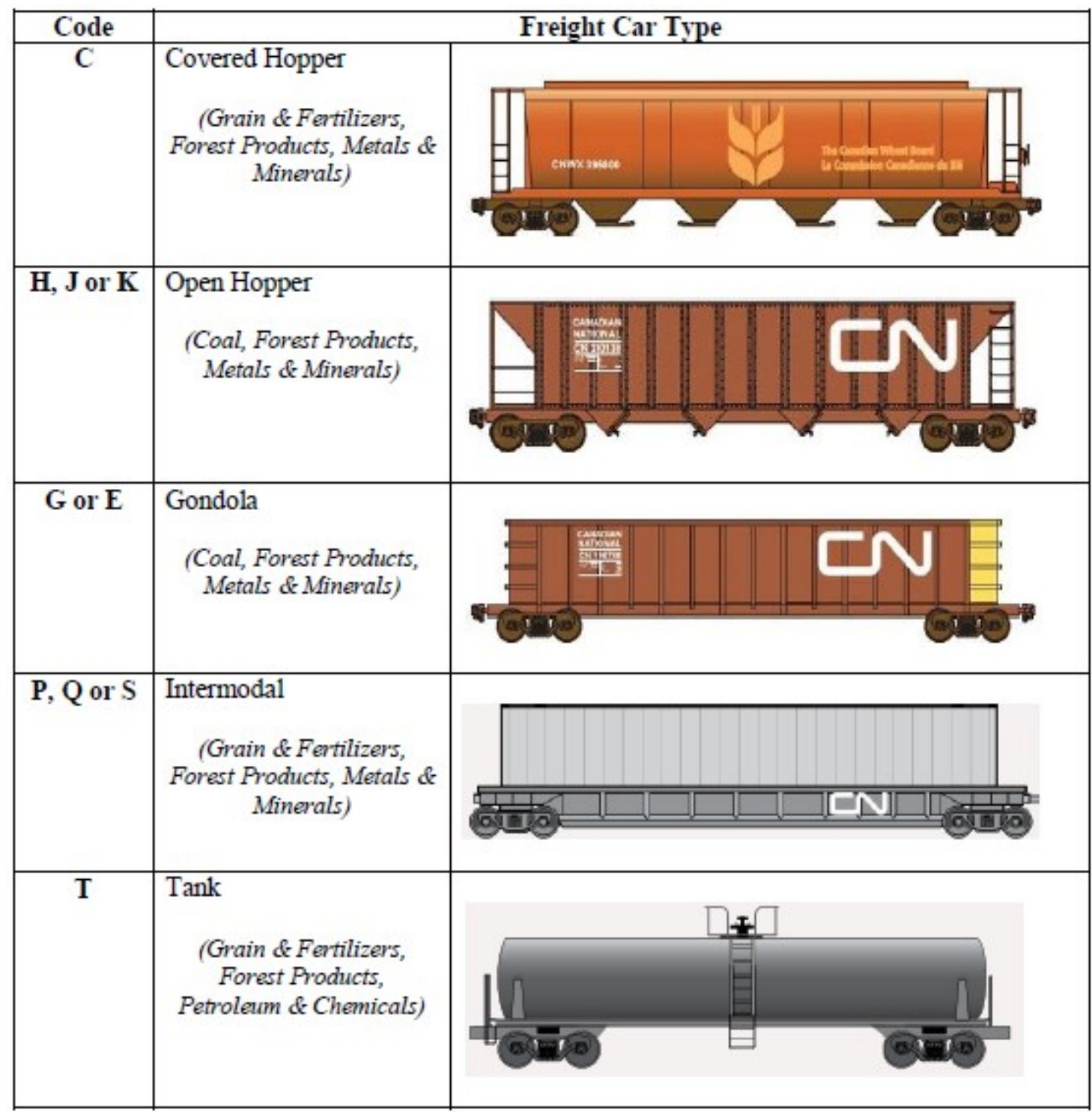

Averaging out the number of freight cars on line, by car type, for the weeks from September 2016 to August 2017 for $\mathrm{CN}$ is shown in Figure 18 [22]. This data will be relied on to aid in the selection of the most frequently used type of freight car for possible redesign and light weighting based on the assumption that the most common car will also contribute to the largest proportion of GHG production [3]. The data shown in Figure 18 is for $\mathrm{CN}$ but the trend is the same for other railway operators [22] [23]. It is obvious that 
the most used car type is covered hopper and thus, this type is chosen as the case study for this thesis.

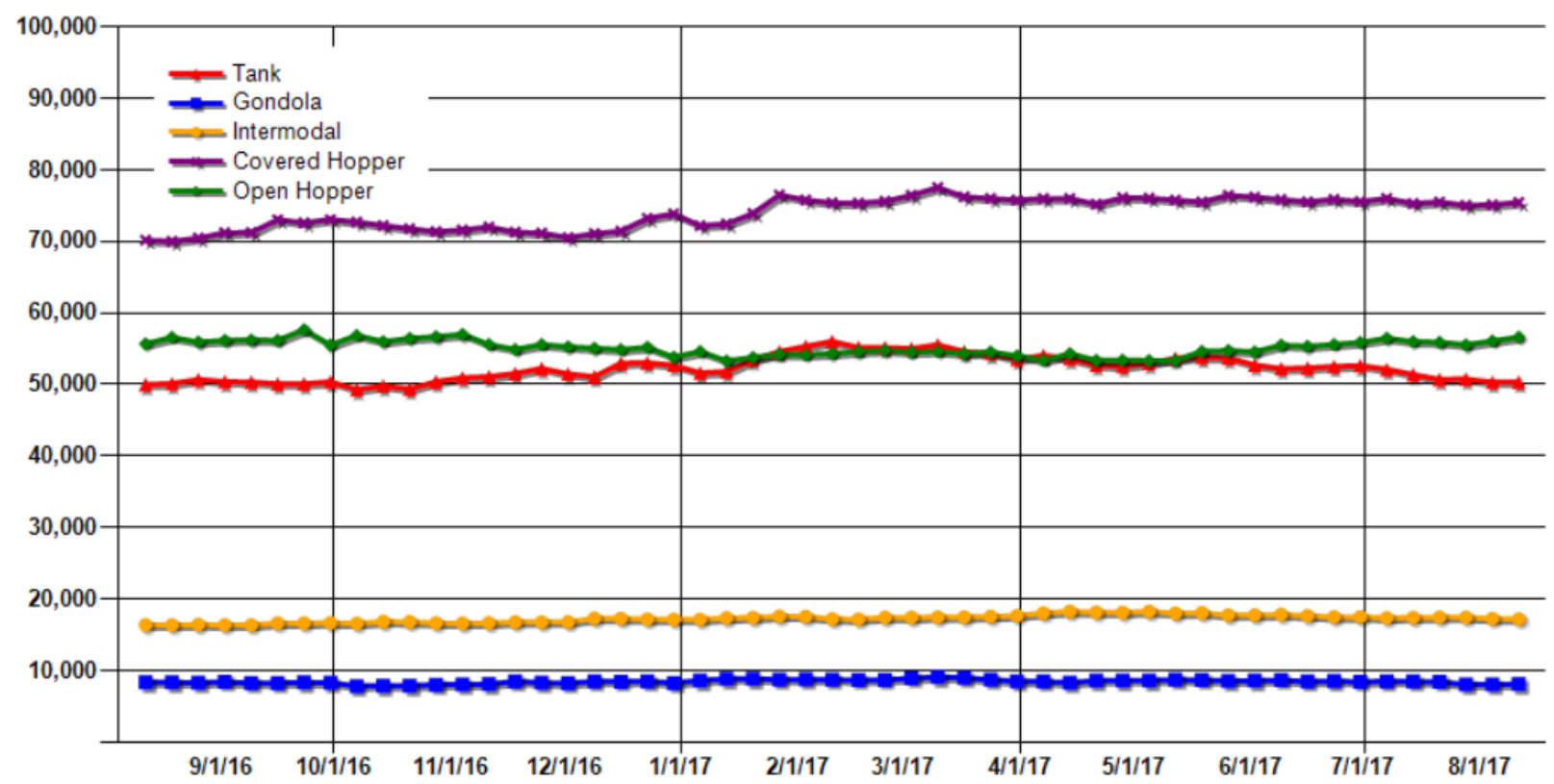

Figure 18: An average weekly number of freight cars on line for CN railways between Sep 2016-Aug $2017[22]$

\subsection{Background Summary}

In this chapter, Canada's share in the global emission, and the necessity of taking actions toward energy saving and emission reduction was explained. For that reason, transportation section was chosen to be investigated because it is one the most important sectors in Canada in terms of GHG production. In the next stage, weight reduction was shown to be one the most effective methods for energy saving and GHG reduction. This step was followed by an introduction to weight reduction strategies, i.e. material substitution and structural optimization. For material substitution, composite materials were introduced, and also different case studies were reviewed. Then, there was an 
introduction to optimization concept. Finally, different aspects of structural optimization were discussed, and previous studies about the light weight design of railcars were reviewed. It was concluded that there is a lack of research on structural design of freight cars. Therefore, the most common type of freight railcars in Canadian rail system (covered hopper cars) was chosen to be the case study for this thesis.

In this thesis, a relatively unexplored application in structural design, i.e. light weight design of freight cars will be studied. For that purpose, a lightweight material should be selected as an alternative to conventional steel. This will be done by using material metrics and considering different parameters like cost, applied loads and manufacturability. After finding the load cases, conducting stress analysis and selecting the material, the railcar panels with their newly selected material will be replace by their optimized sandwich versions to achieve the highest possible weight reduction. 


\section{Chapter 2. Model Preparation and Stress Analysis}

As discussed in Chapter 1, this project aims to investigate both structural optimization and material substitution to achieve most possible weight reduction. To properly select the material, it is necessary to be aware of the magnitude of the stresses, the location of high stress concentrations and the type of loading that is applied to the selected covered hopper railcar. In this chapter, these parameters are described. However, to conduct stress analysis, the CAD model of the selected railcar is needed. Therefore, it is required to create a $\mathrm{CAD}$ model before starting the structural design procedure.

First, some basic terminology and components in the rail industry are introduced. Then, the geometrical and dimensional information of a sample covered hopper railcar will be presented. This information will be used to create the CAD model. In the next section, the applied loads on a covered hopper railcar body will be discussed according to AAR standards [6]. Then, load cases for a covered hopper car will be defined, and the CAD file will be imported to ABAQUS for finite element stress analysis. This step consists of explaining boundary conditions, mesh density and discussion on results. Finally, some other simulation techniques, which are different from what is proposed by the AAR manual [6], will be introduced.

\subsection{Structural Components and Terminology}

Before discussing the design of freight railcars, it is necessary to introduce some structural components and define technical terms related to rail car design. Some of these structural components are presented in Figure 19. 


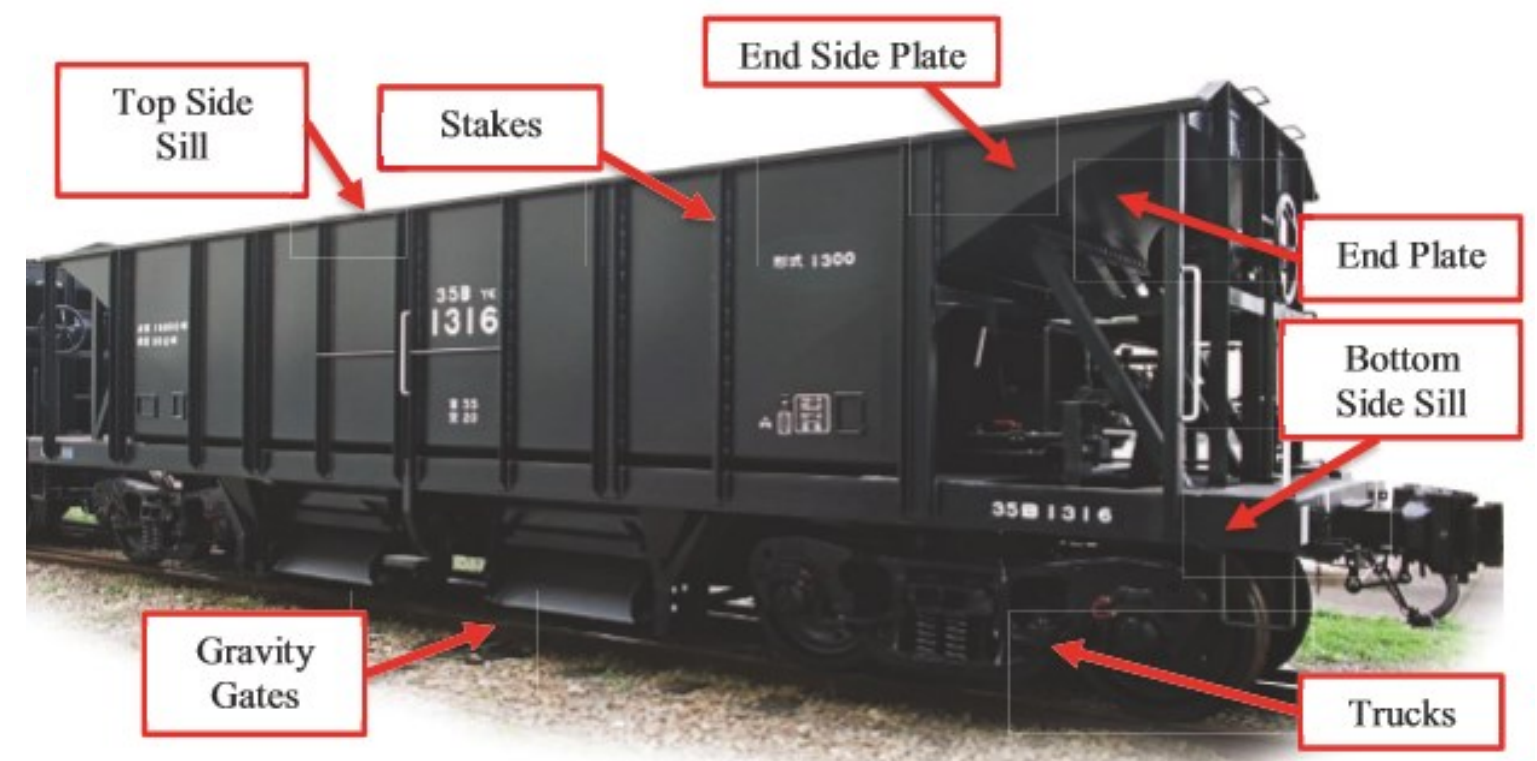

Figure 19: Main components of covered hopper car [3]

- Truck (Bogie): Rail car wheel assembly usually having two or more axels. They pivot freely beneath railroad cars in order to allow them to navigate turns [3]. Each bogie contains the railcar suspension system and many other under frame structural components, as seen in Figure 20.

- Stakes (Stiffeners): The side stiffening pieces used to hold the load [3].

- Sills: The main longitudinal members that are connected transversely by the end sills, body bolster and cross ties. Sills are divided into side sills, intermediate sills and center sills [3].

- Side sills: The outside longitudinal members of the underframe [3].

- Center sills: The central main longitudinal members of the underframe of a car that are usually located in the center of the car, and transmit most of the biffing shocks from end to end of the car [3]. 


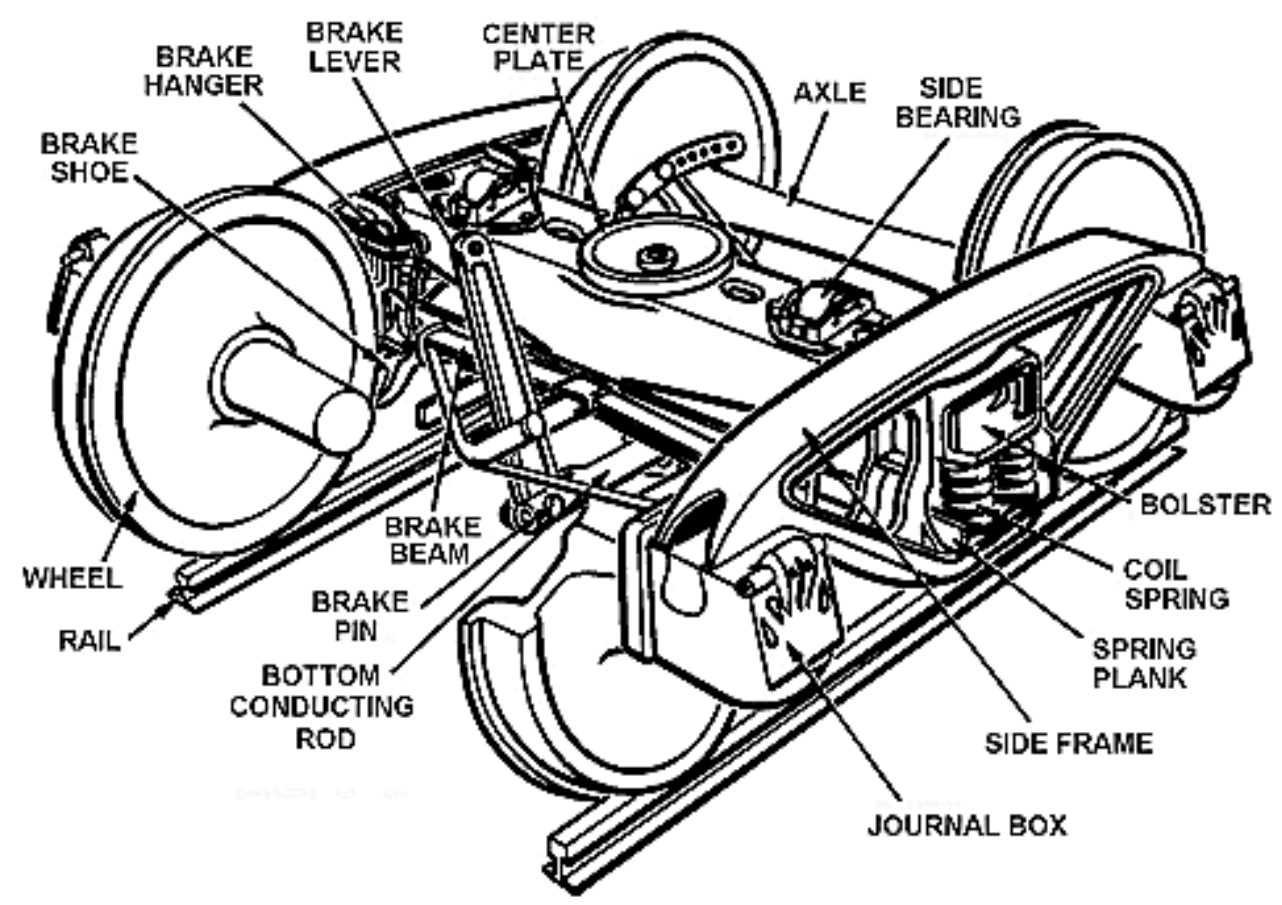

Figure 20: Underframe components in bogie [24]

There are also technical terms that need to be defined according to the AAR manual [6]:

- Light weight: Total weight of the empty car including trucks and all appurtenances considered part of the car.

- Maximum gross weight on rails: Light weight of car plus the maximum weight of the lading.

- Load limit: Maximum weight of lading, and the difference between the stenciled light weight of car and the maximum weight on rails.

- Live load: Equal to the load limit or any percentage specified for design load.

- Dead load: Weight of car body structure, all fixtures permanently attached and all appurtenances considered part of the car. For stress analysis, it shall be 
permissible to use the estimated light weight of the complete car less the weight of the trucks.

\subsection{Geometrical Information}

The very first step through redesigning conventional railcars is to create a CAD model of the selected type railcar. This will be required for further stress analysis, material selection and structural optimization. For that purpose, one of the BNSF RAILWAY [25] covered hopper cars (ASTF 350100-350349) was selected as the case study for this research project. Geometrical information for this car is shown in Figures 21 and 22. More detailed geometrical information is also listed in Table 2. It must be mentioned that the North American rail industry use US units but in this thesis, both US and SI units are presented for geometrical data. However, in this thesis, further calculations and stress analysis will be based on SI units. The other point is that although with US units dimensions are normally given in terms of "inches", the rail industry uses a different convention, i.e. combination of both "feet" and "inches" similar to architectural and building design practices, and this is presented in the Table 2 as given in the reference document [24].

Table 2: Detailed geometrical information of BNSF covered hopper car [24]

\begin{tabular}{|c|c|}
\hline Coupled length & $41^{\prime}-101 / 2 "(12763.5 \mathrm{~mm})$ \\
\hline Truck centers & $28^{\prime}-4 "$ " $(8636 \mathrm{~mm})$ \\
\hline Inside width & $9^{\prime}-115 / 8^{\prime \prime}(3038.48 \mathrm{~mm})$ \\
\hline Extreme width & $10^{\prime}-8^{\prime \prime}(3251.2 \mathrm{~mm})$ \\
\hline Extreme height & $15^{\prime}-1 "(4597.4)$ \\
\hline
\end{tabular}




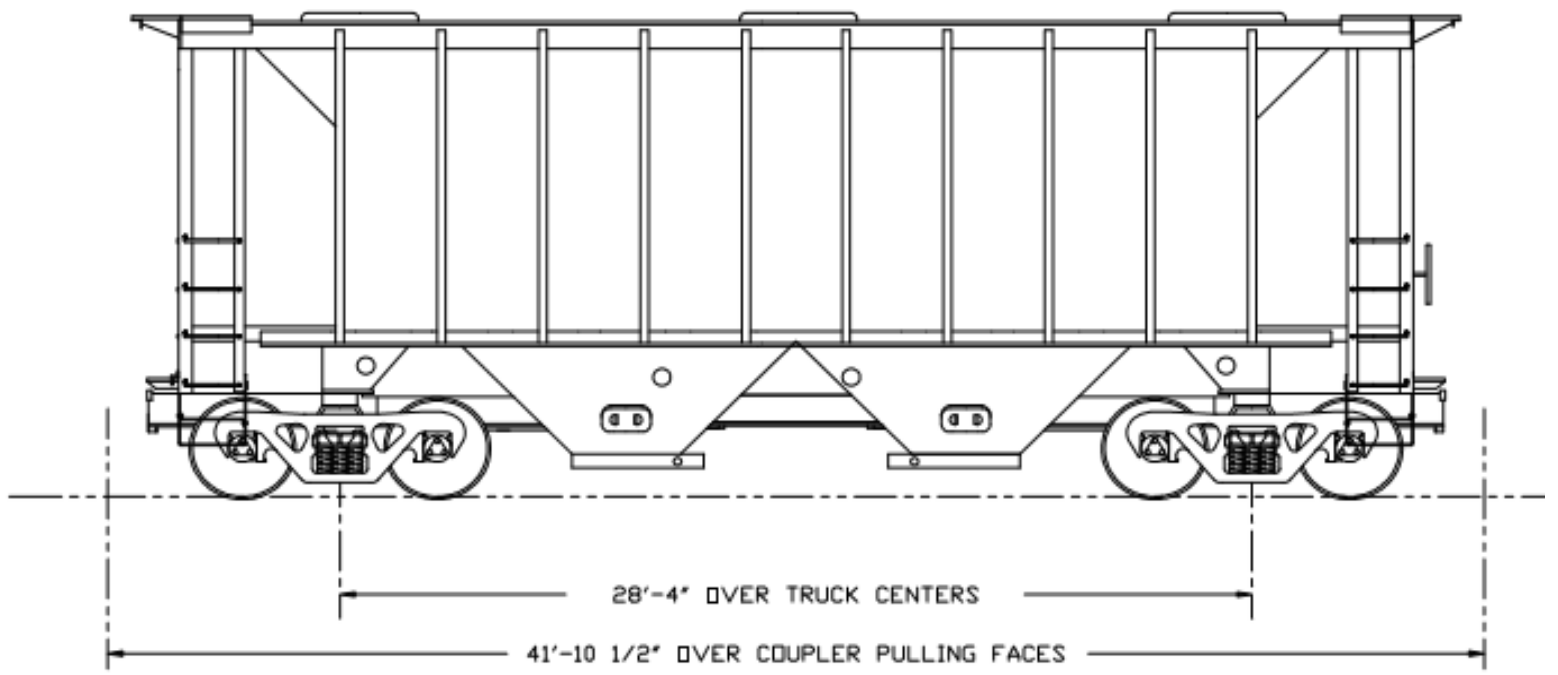

Figure 21: Side view of the BNSF covered hopper railcar [24]

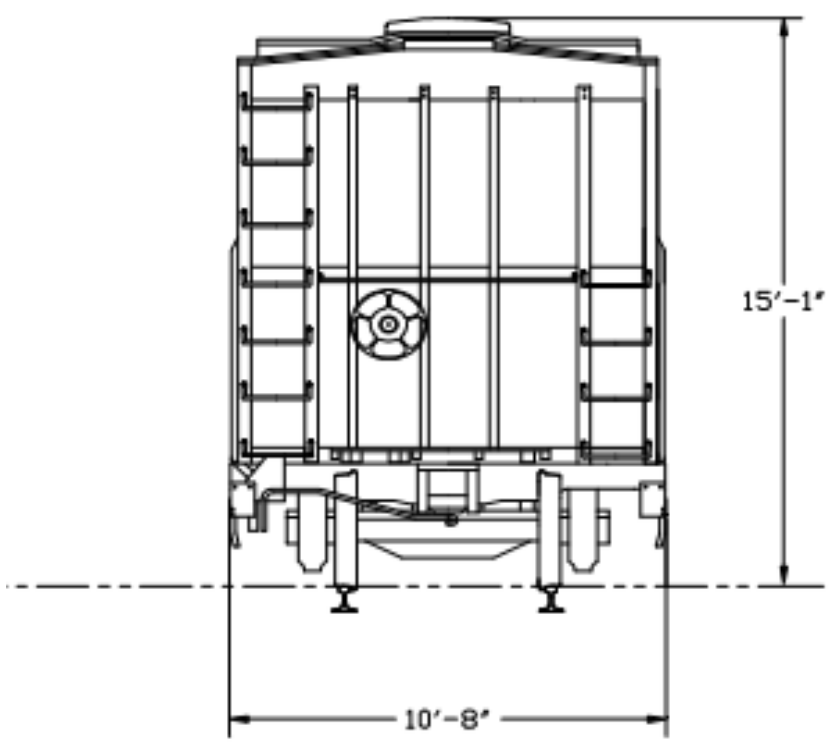

Figure 22: Front view of BNSF covered hopper railcar [24]

To sketch the CAD model, more detailed information on the railcar cross section is needed. This can be found in the AAR manual, standard S-2026 [26]. Different types 
of cross sections (plates) are introduced in AAR standards, and categorized based on their truck center, extreme height and extreme width [26]. By comparing the dimensions of the BNFS covered hopper car with different plates introduced in the AAR manual, it is concluded that the plate type B matched with this case study. The detailed information of the plate type B can be found in Figure 23.

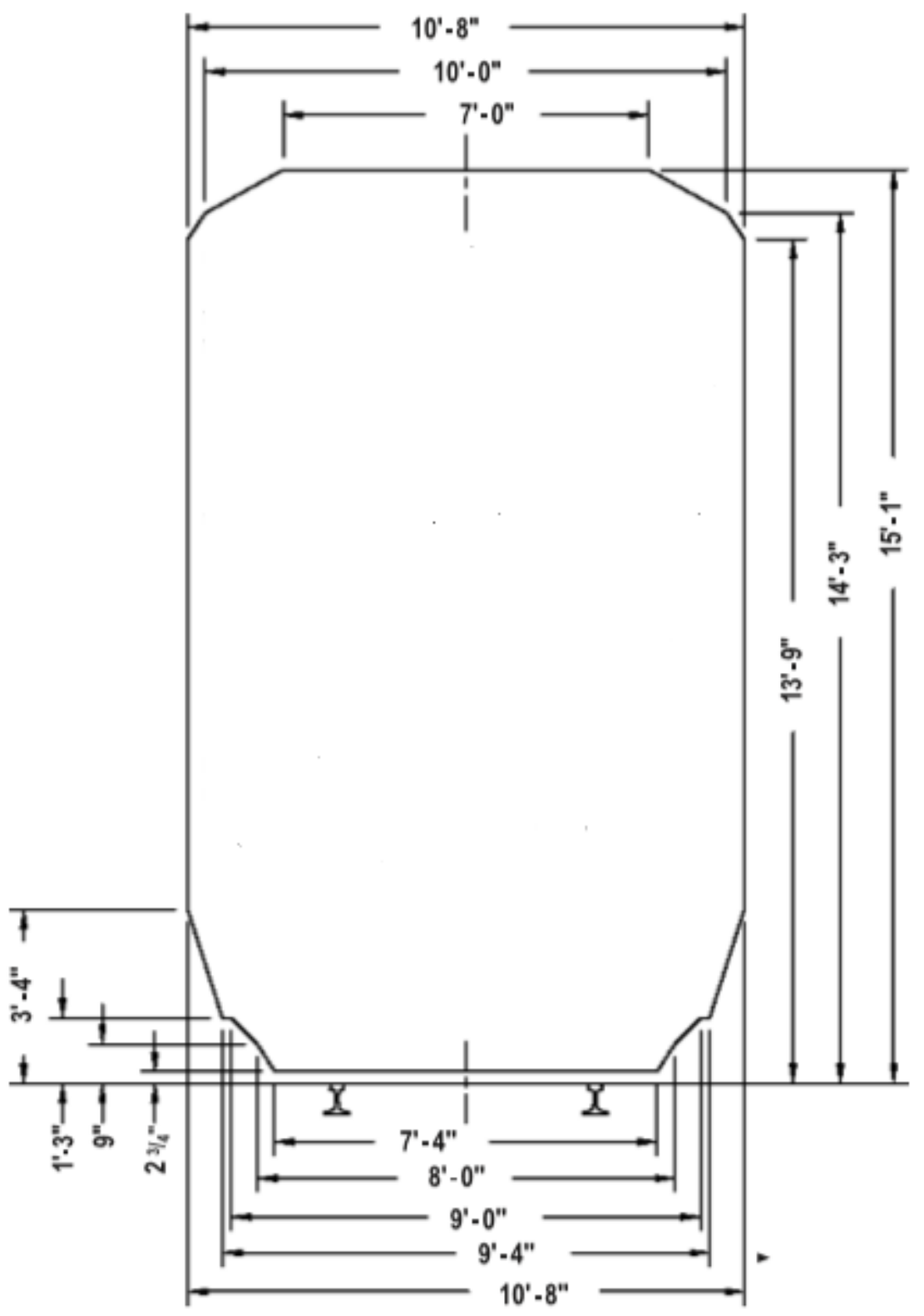

Figure 23: Plate B clearance requirements [26] 
In addition to what was mentioned, according to the specification M-908, section 4.1, the smooth floor sheet is to be a minimum thickness of 10 gauge $(2.59 \mathrm{~mm})$ [26]. This specification does not apply to floors where the floor plate is attached directly to the underframe members [6].

\subsection{Development of the CAD Model}

It should be mentioned again that the focus of the project is only on the car body itself not the supporting members or underframe structure. By using the mentioned geometrical information, the simplified CAD model of the BNSF cover hopper car was created using CATIA V5. This can be seen in Figure 24 and 25.

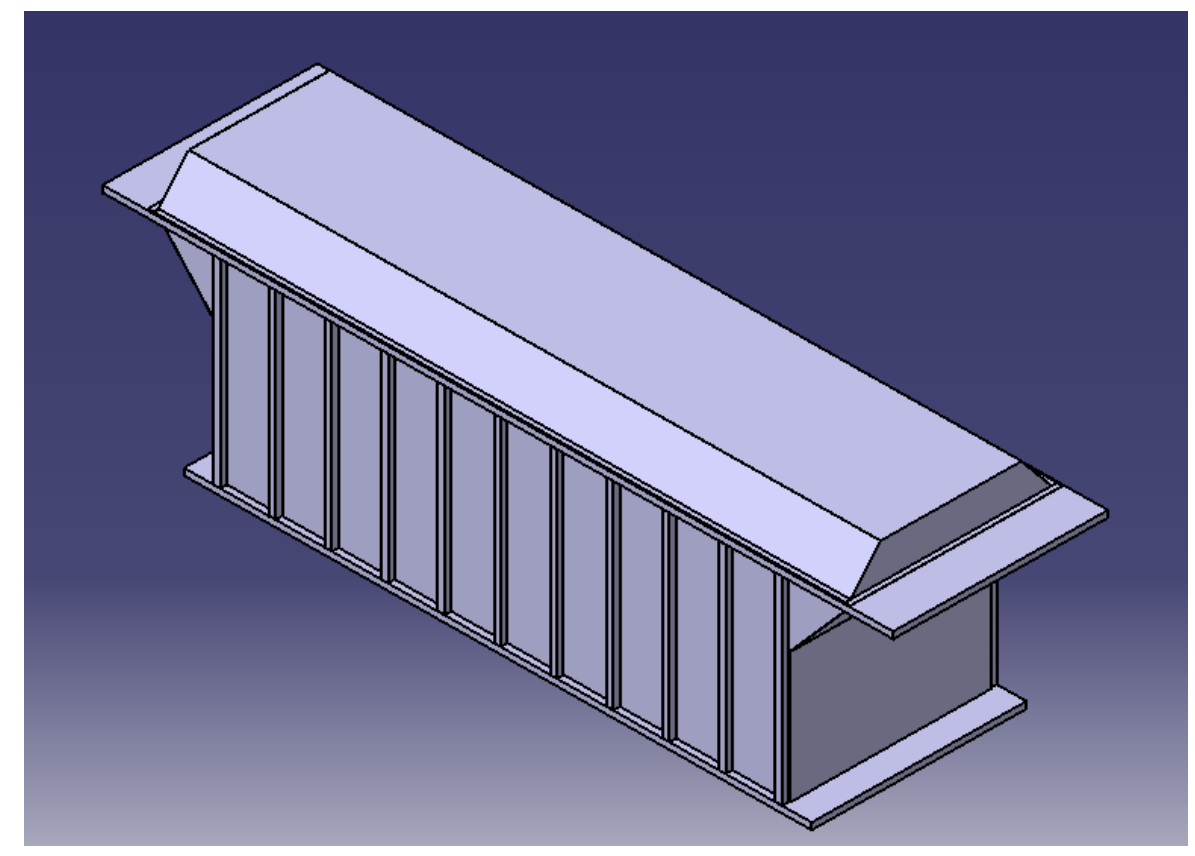

Figure 24: CAD model of the BNSF covered hopper railcar body 


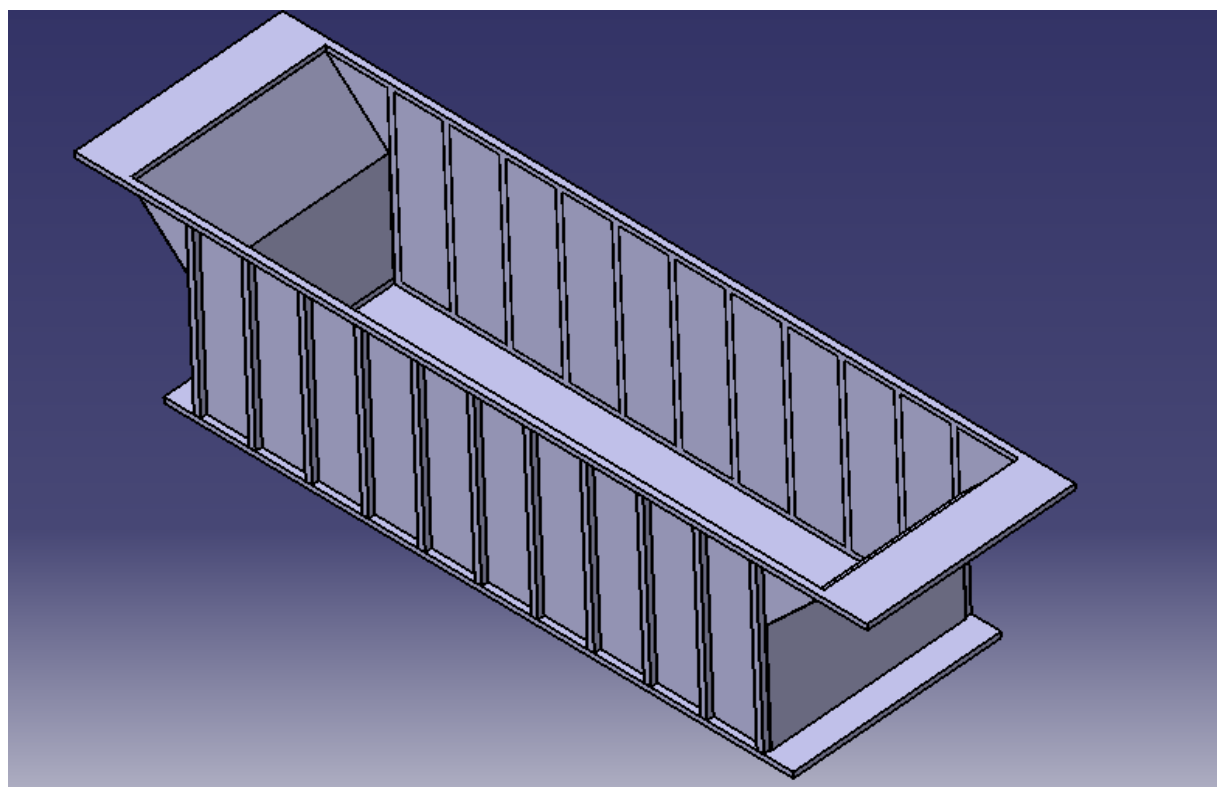

Figure 25: Inside view of the BNSF covered hopper railcar body

As was mentioned, here, the model has been simplified for this analysis. In fact, in real covered hopper rail cars, the roofs typically have several hatches which each have their own specific geometrical, fatigue and attachment requirements that are different from the rest of body and can be analyzed in a future research project. Besides, the roof panel does not play an important role in load carrying capabilities of the car, and therefore, it was decided to only analyze the body. The mentioned hatches and some other special features of the real covered hopper railcar roof can be seen in Figure 26. Of course, the details of the created model are defined according to research goals, i.e. almost all underframe members and other upper frame supporting structures were eliminated, since the research is focused only on the railcar body and its load carrying capabilities. Some of members like side sills might appeared to be upper frame members but according to the AAR Manual, they are categorized as underframe members [6]. 
After creating the CAD model, the next step would be stress analysis. For that purpose, first, different load cases for a covered hopper railcar have to be defined.

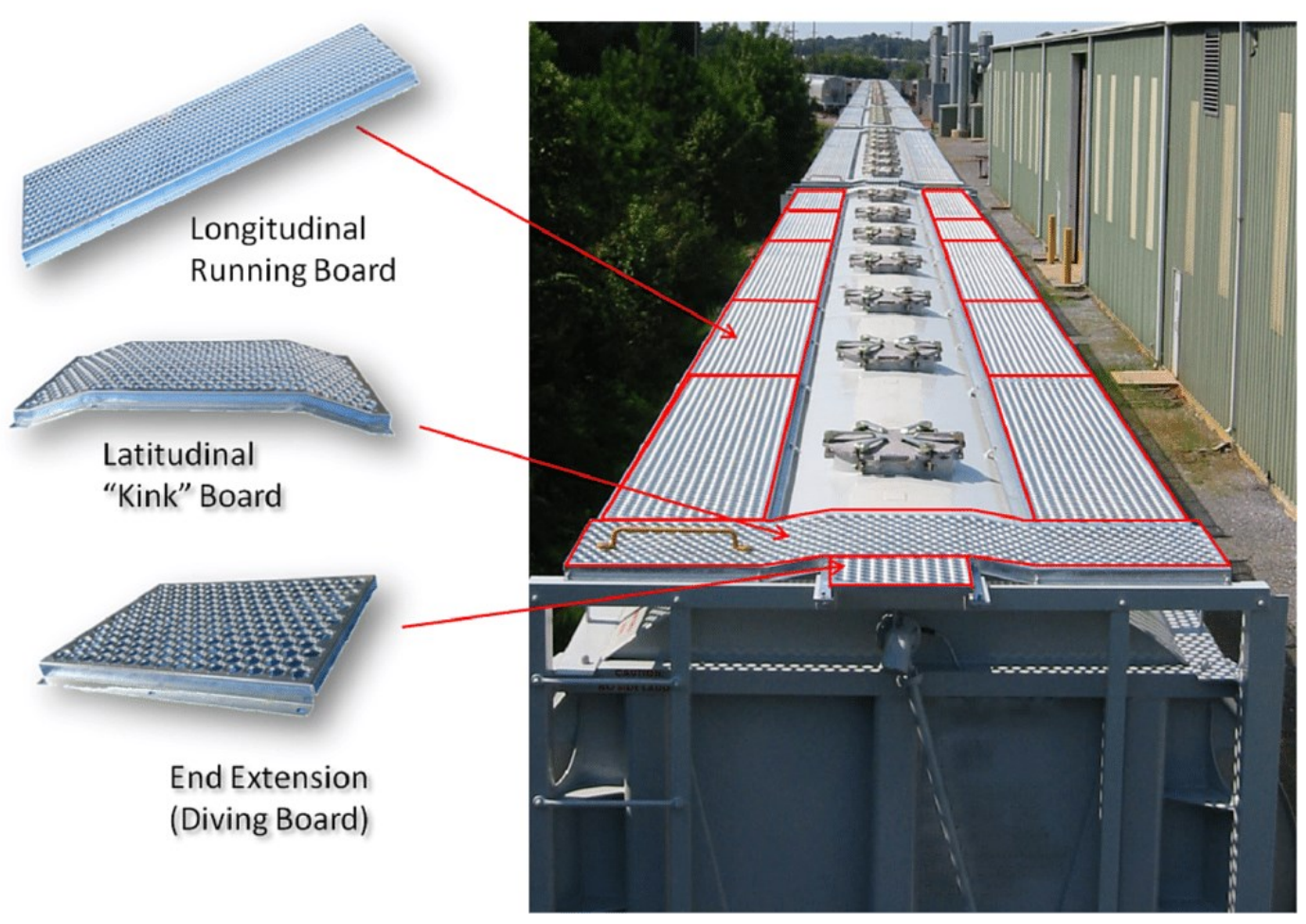

Figure 26: Roof of covered hopper car [27]

\subsection{Loads and Forces}

There are different types of loads that might be applied to a railcar structure. However, this depends on the type of the selected railcar and also the target structure. In fact, some of the loads are carried by underframe structure, while others are applied to the body or even supporting structures. Also, some of the loads are only applied to specific types of railcars and would not be the case for other ones. This is something that must be considered in structural design of a railcar. In this project, the target structure is the body of the covered hopper railcar. According to AAR standards [6], the body is responsible 
for carrying the cargo load, i.e. live load. This load has different forms on different body panels, which can generally be categorized as vertical loads and lateral loads.

Before discussing about lateral and vertical loads, it is necessary to specify the load capacity and load configuration in the selected covered hopper car. Many different load configurations for different types of railcars are introduced in the AAR Manual [6]. Generally, there is no strict regulation for choosing the load distribution and it depends on the type of application but in the case of hopper cars, they must be loaded to their full capacity. Therefore, no variable load configurations were introduced for these type of railcars, as they should have $100 \%$ uniform load distribution. However, to better illustrate this, a similar load distribution case for box cars is shown in Figure 27.

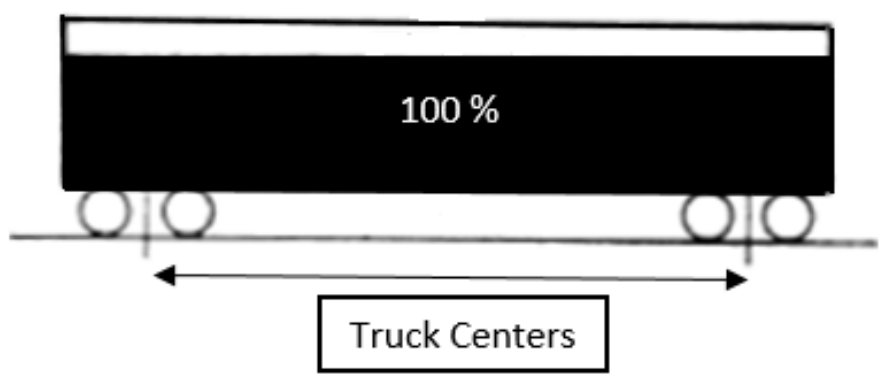

Figure 27: Uniform full capacity load distribution in a box car [6]

\subsubsection{Vertical Loads}

There is a general classification for nominal capacity of rail cars in the AAR manual. This classification relates the nominal capacity of a typical hopper railcar to its maximum gross weight. This is listed in Table 3 . 
Table 3: Classification of railcars based on their nominal capacity [6]

\begin{tabular}{|c|c|}
\hline $\begin{array}{c}\text { Nominal Capacity } \\
\text { (US ton) }\end{array}$ & $\begin{array}{c}\text { Maximum Gross Weight } \\
\text { (lb) }\end{array}$ \\
\hline 70 & 220,000 \\
\hline 100 & 286,000 \\
\hline 125 & 315,000 \\
\hline
\end{tabular}

The load carrying capabilities of the selected BNSF hopper car is also listed in Table 4.

Table 4: Capacity related info on the selected BNSF railcar [25]

\begin{tabular}{|c|c|c|}
\hline Load Limit (lb) & Gross Weight (lb) & Light Weight (lb) \\
\hline 205,700 & 263,000 & 57,000 \\
\hline
\end{tabular}

First, although the initial CAD model was created based on the BNSF hopper car, the design proposed in this thesis can be adapted for a different class of hopper cars, i.e. it does not necessarily have to follow all the characteristics of the selected BNSF hopper car. In fact, new railcar designs are assessed based on AAR standards [6], not their compatibility with previous models fabricated by different manufacturers. Second, new railcars are not rejected because of their introduced nominal capacity and load limit. The only thing matters is that the designer should demonstrate the ability of the new railcar to carry the introduced load limit without any structural failure.

Nominal capacity is known as any capacity based on the car's light weight, journal size and gross weight on rails, and in no event may the nominal capacity exceed 
the load limit [6]. The light weight was defined as the total weight of the empty car including trucks and all appurtenances considered part of the car [6]. According to definitions, it is understood that the load limit (or full nominal capacity) of a rail car can be applied when detailed information on all panel thicknesses, cross sectional profiles, layout of stakes and a complete railcar structure including underframe and all other appurtenances are given. Since many of this data are not available, it would be unrealistic to apply a load limit (or full nominal capacity) of a complete railcar to another body which most of its underframe and supporting structures are eliminated, and the layout and geometry of the rest are estimated. Even the geometry of the created CAD model is different to some extent from the BNSF railcar. In fact, the geometrical data of the BNSF model was used as a guideline for creating a new CAD model, but the new design is different from the BNSF railcar. Therefore, the proposed design does not have to exactly follow the BNSF given data, it means different nominal capacity and load limit are defined by the author for this new railcar. Following the regulations of the AAR [6], this new railcar is categorized as 70 US ton (63.5 metric tons) nominal capacity railcars. The load limit for this car is considered as 70 metric tons ( 78 US ton) and would be the vertical load case for this study.

The schematic distribution of the live load (vertical load) is shown in Figure 28. 


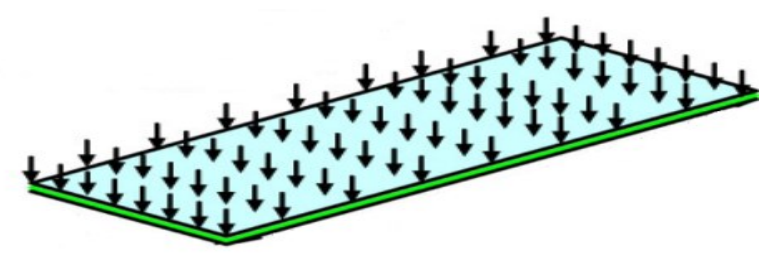

Figure 28: Live load distribution on the floor panel ${ }^{1}$

It has also stated that for stress analysis, it shall be permissible to use the estimated light weight of the complete car less the weight of the trucks [6]. This can affect the gross weight and its corresponding nominal capacity. This assumption can even better justify the use of 70 US ton nominal capacity for the BNSF car.

\subsubsection{Lateral Loads}

Lateral pressure of granular, lump or pulverized bulk material shall be considered in the design of the containing structure of all cars in which such pressures may be active, e.g. box, hopper and gondola cars. For closed-top cars the lateral pressure may be computed by the following Rankine formula [6]:

$P=0.5 W h^{2}\left(\frac{1-\sin \varphi}{1+\sin \varphi}\right)$

Where;

$P=$ Lateral force $(\mathrm{N} / \mathrm{m}$ of length)

$W=$ Weight of lading $\left(\mathrm{N} / \mathrm{m}^{3}\right)$

$h=$ Depth of lading - not including heap (m)

$\varphi=$ Static angle of repose $\left(25^{\circ}\right)$

\footnotetext{
${ }^{1}$ iLLStreet Composites, "Carbon Fibre Sheet Deflection", [Online]. Available: http://www.carbonfiberglass.com/Carbon-Fiber-Sheets-Composite-Panels-Fibre/Carbon-Fiber-SheetTechnical-Data/carbon-fiber-sheet-deflection
} 
The lateral force is to be distributed vertically so that it is at maximum at the floor line decreasing uniformly to zero at the top surface of the lading [6]. This would be the load distribution on both side and end walls in this thesis.

In case of dynamic analysis, the effects of centrifugal force and/or acceleration shall be considered. For this computation, a lateral acceleration of $0.3 \mathrm{G}$ shall be used [6].

\subsubsection{Load Factors}

There are other types of loads such as buff (compressive), draft (tensile) and jacking loads that a rail car structure might experience. These loads act on structures such as bottom side sills, center sills and body bolster. In fact, the previously explained lateral and the vertical forces are the only types of loading that directly applied to the body. The AAR has proposed different load factors for different loading cases, i.e. different combinations of possible loads. However, this thesis is not intended to study the underframe structures and their corresponding loads. Only the introduced lateral and vertical loads are investigated here, and therefore, regardless of other types of loading, the most conservative case (highest load factors) will be chosen for this project. "[That is] where the design of a member is governed by lateral forces singly or in combination with a dead load, live load and buff and draft loads. In this case, the load factor for the lateral forces shall be 1.5 and for the other loads it shall be 1.8 " [6].

\subsubsection{Load Cases}

In contrast to other structures like airplane, where different load cases with their own load factors must be studied separately, in rail car design the harsh load conditions might occur at the same time, i.e. a rail car might move on a bumpy, slopped path where it is necessary to apply both vertical and lateral load factors to take the effect of harsh 
conditions on different panels into account. Therefore, the mentioned load factors have to be applied simultaneously. There is also another case, which can be called as "base loading case", where a freight car moves on a smooth, straight path and there is no need to apply any load factors. However, the base case cannot be the basis of the design as its load magnitudes are smaller compared to possible harsh conditions. In fact, if the structure is designed according to the base case, there would be a high probability of failure when the car is subjected to harsh loading conditions.

To summarize, the rail car will be designed according to harsh loading conditions, and both lateral and vertical load factors will be applied at the same time. However, the type of analysis which will be conducted in this section, is linear static stress analysis. This is the recommended initial design phase for further steps like fatigue life prediction, material selection or structural optimization. In fact, only static loading is considered, and the effect of centrifugal force is not discussed in this research, but this can be investigated in future studies.

\subsection{Finite Element Simulation}

In this section, the CAD model was imported to ABAQUS for stress analysis. This section describes model preparation steps like meshing, selecting the element type and boundary conditions.

\subsubsection{Mechanical Properties}

One of the early steps in the finite element analysis process is to define the material properties. It is known that the majority the conventional railcars are made of steel. However, it seems that the exact mechanical properties of the steel and even the 
type of steel that rail car manufacturers use, considered as proprietary data, and is not available. Therefore, in the first phase of the current project, "A preliminary study on the use of FRP" [3], a piece was cut from a decommissioned steel hopper railcar, different small samples were cut and tested and the average mechanical properties were obtained by the author. The piece cut from the sidewall panel was almost $7 \mathrm{~mm}$ thick, and the mechanical properties are listed in Table 5. In this thesis, these properties will be used for FE simulation.

Table 5: Mechanical properties of a steel hopper car [3]

\begin{tabular}{|c|c|c|}
\hline Mechanical Property & Average & Selected Value for FEA \\
\hline Young Modulus (GPa) & $209 \pm 1.4$ & 210 \\
\hline Poisson's Ratio & $0.28 \pm 0.01$ & 0.28 \\
\hline Tensile Strength (MPa) & $537 \pm 8.5$ & 545 \\
\hline Yield Point (MPa) & $448 \pm 8.2$ & 455 \\
\hline
\end{tabular}

The stress stress-strain curve of one of the test samples is shown in Figure 29.

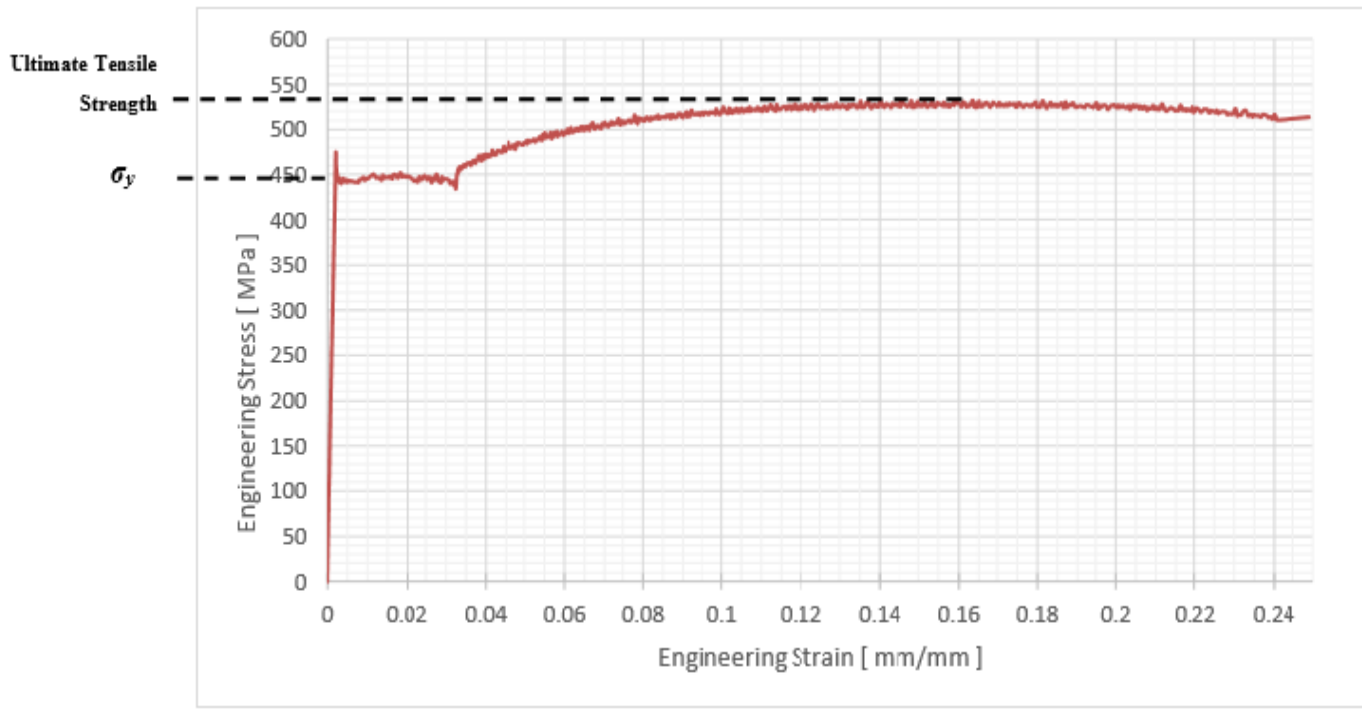

Figure 29: Engineering stress-strain curve of the unknown sample steel [3] 


\subsubsection{Element Selection}

According to AAR standards, most of freight car structures should be modeled using plate or shell elements [6]. They adequately represent thin-plate materials where through-thickness properties are not critical to the design. When such properties are critical, i.e. when plate thicknesses are of $5 / 8$ in. or greater (16mm and above), and significant through-thickness stresses can occur, solid elements should be used [6]. Nonetheless, this is not the case for the railcar body which is made of thin plates with 7 $\mathrm{mm}$ thickness. Therefore, it makes sense to model the body with shell elements, as suggested by the AAR method. According to standards [6], shell elements should be a minimum of four node quadrilateral with six degrees of freedom at each node. Membrane and bending stresses must be adequately represented by the element. This type of element does not perform well when allowed to degenerate into its triangular form, i.e. stiffer than quadratic elements, and this should be avoided in regions where fatigue stresses are to be

calculated [6]. As the railcar body will be further simulated in ABAQUS, it is necessary to study its element library to find the element type which can satisfy the AAR regulations [6].

There are several types of planar elements provided in ABAQUS. For structural applications these include plane stress elements and plane strain elements. Plane stress elements can be used when the thickness of a body or domain is small relative to its lateral (in-plane) dimensions. The stresses are functions of planar coordinates alone, and the out-of-plane normal and shear stresses are equal to zero. Plane stress elements must be defined in the $\mathrm{x}-\mathrm{y}$ plane, and all loading and deformation are also restricted to this plane. This modeling method generally applies to thin, flat bodies [29]. Plane strain 
elements can be used when it can be assumed that the strains in a loaded body or domain are functions of planar coordinates alone and the out-of-plane normal and shear strains are equal to zero. Plane strain elements must be defined in the $\mathrm{x}-\mathrm{y}$ plane, and all loading and deformation are also restricted to this plane. This modeling method is generally used for bodies that are very thick relative to their lateral dimensions [29]. According to the above, the plane-stress elements are suitable for simulating the railcar body which can satisfy the AAR regulations [6]. However, there are two general type of plane-stress elements available in ABAQUS: membrane elements and shell elements.

Membrane elements are used to represent thin surfaces in space that offer strength in the plane of the element but have no bending stiffness, e.g. the thin rubber sheet that forms a balloon. In fact, membrane elements are surface elements that transmit in-plane forces only (no moments), and have no bending stiffness. On the other hand, shell elements can represent bending stiffness in addition to in plane strength [29]. In fact, shell elements are based on a reduction of solid elements and can represent both inplane and out-plane forces. This makes them suitable for simulating planes under bending loading, which is the case in this thesis.

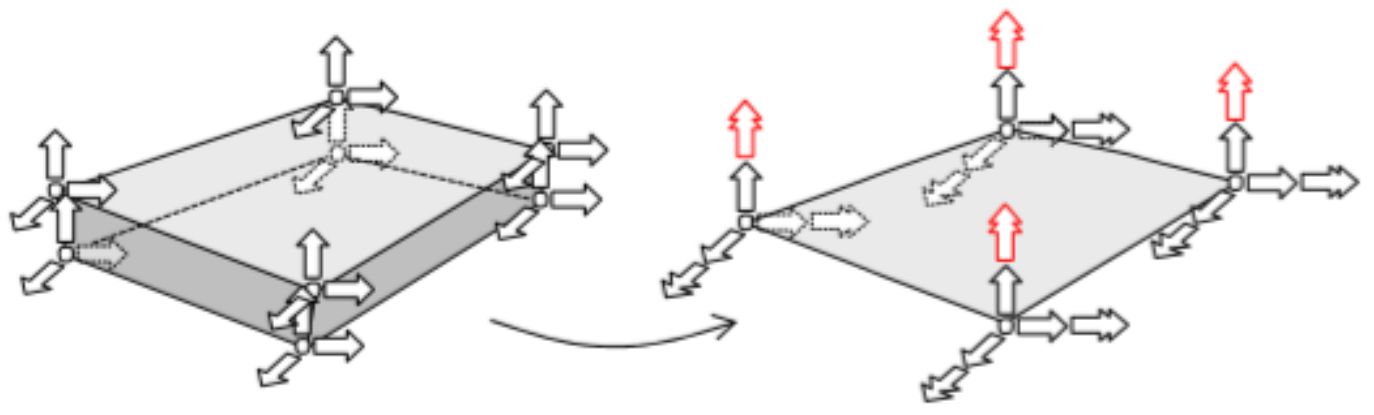

Figure 30: Degrees of freedom of a shell element [30] 
Another issue that must be pointed out is whether to use the linear 4 node shell elements or higher order 8 node elements. The answer to this question lies on the properties of these two elements in ABAQUS library [29]. In fact, linear 4 node shell element (S4R) is a general-purpose, conventional shell element which provide robust and accurate solutions to most applications, while second order 8 node shell element (S8R) only used for thick shell problems where the thickness is more than about $1 / 15$ of a characteristic length on the surface of the shell, such as the distance between supports for a static case [29]. Therefore, the use of higher order elements is not appropriate for this case, as it is more computationally expensive and is not even necessary according to the AAR standards.

The term S4R, refers to shell type (S), 4 nodes (4) and reduced integration (R) element. Many shell element types in ABAQUS use reduced (lower-order) integration to form the element stiffness matrix. The mass matrix and distributed loadings are still integrated exactly. Reduced integration provides accurate results and significantly reduces running time, especially in three dimensions [29]. In fact, in all finite elements the material behavior (stresses, stains, yielding, cracks, etc.) is computed in several points. These points are called integration points or Gauss points. The stresses etc. in other points of the element are computed by interpolation and extrapolation [30]. 


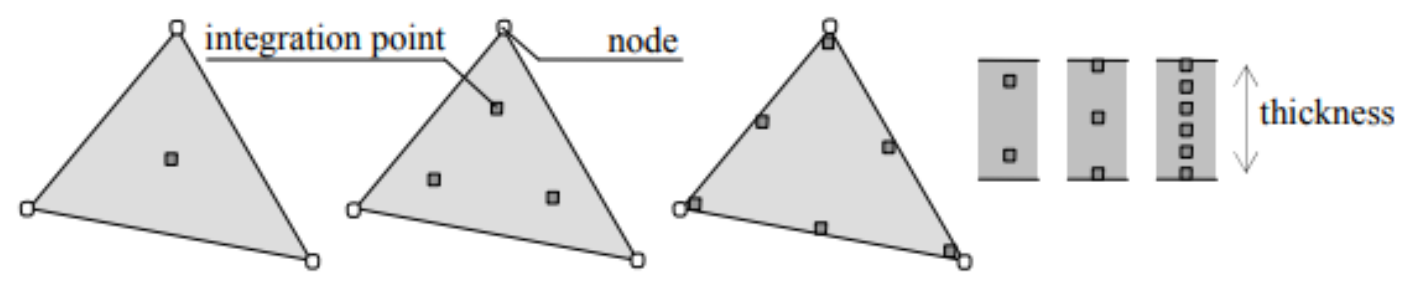

Figure 31: Possible locations of integration points in triangular elements [30]

Due to reduced integration the elements may have no stiffness at all for deformations. Consequently, the elements can deform in a pattern that looks like an hourglass. This mode is shown in Figure 32. This deformation is called an hourglass mode and is not desired. However, an hourglass mode can only occur in a perfectly regular mesh with special boundary conditions. In a practical finite element model these hourglass modes are extremely rare [30]. Though this is not a common case in FE simulation, when reduced integration is used in ABAQUS with first-order (linear) elements, hourglass control is required to make sure this mode is prevented [29].
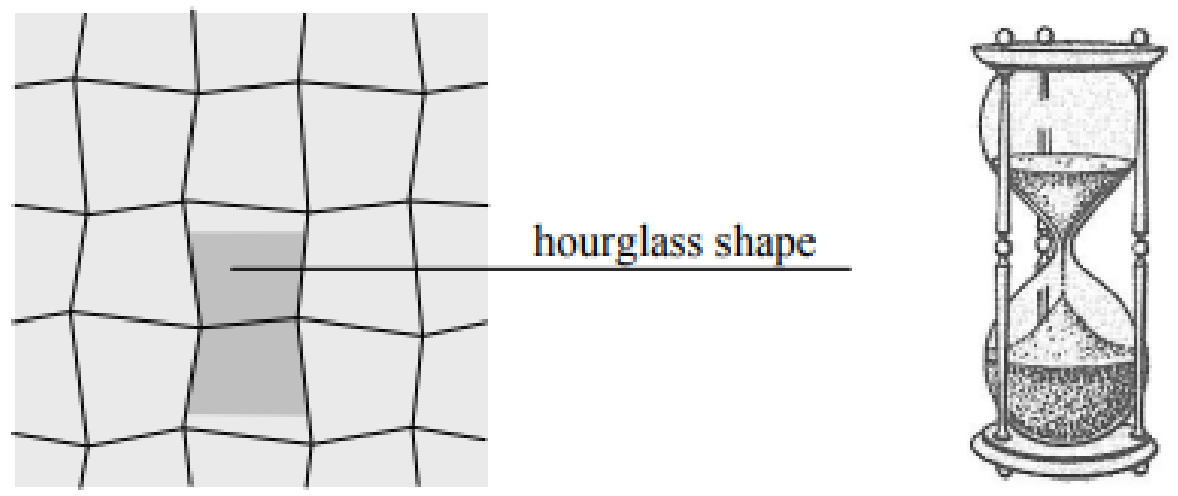

Figure 32: Deformation of square elements into an hourglass mode [30] 


\subsubsection{Boundary Conditions}

A set of recommendations for applying the boundary conditions are introduced by the AAR manual [6]. This is described below:

"The model boundary conditions must not constrain the structure more than would the real car's actual support. They should provide constraints that make the model stable and prevent rigid body motion without constraining the model in any other way. This generally implies single node constraints at support locations. The distribution of load across an area at support locations should generally be achieved by applying those loads explicitly and not by restraining an area of nodes by using boundary conditions" $[6]$.

"A freight car is supported on its truck center plates and side bearings, and these are, in turn, supported by the primary suspension. There is no pitch restraint introduced by this arrangement, and so no moment restraint in this plane should be introduced by the boundary conditions. The center plate constraints the vertical and lateral displacements, while the side bearings only provide vertical support. These degrees of freedom have to be constrained by boundary conditions" [6].

As can be seen in Figures 33 and 34, for many hopper cars, like this case study, the car body is not directly attached to its suspension system. It is attached through another structure called the "jack pad". Therefore, although this thesis is not intended to study other supportive structures, it is necessary to model some of them to apply the appropriate boundary conditions based on the AAR Manual [6]. Otherwise, applying nodal boundary conditions to a large unsupported panel of the car would not be feasible, and leads to very high, unrealistic stresses. Therefore, a nodal boundary condition for the 
lateral and vertical constraint $\left(\mathrm{U}_{1}=\mathrm{U}_{3}=0\right)$, and another pair of vertical constraints $\left(\mathrm{U}_{3}=0\right)$ for side bearings are set on the bottom face of the jack pad structure, where it is attached to the underframe, and the vertical reaction forces results from the weight of lading, are equally and uniformly distributed on their corresponding contact surfaces. Lateral forces are in balance, and there is no need to apply lateral reaction forces. It has to be mentioned that $\mathrm{U}_{1}, \mathrm{U}_{2}, \mathrm{U}_{3}$ are translations in $\mathrm{x}$ (lateral), $\mathrm{y}$ (longitudinal) and $\mathrm{z}$ (vertical) directions, respectively.

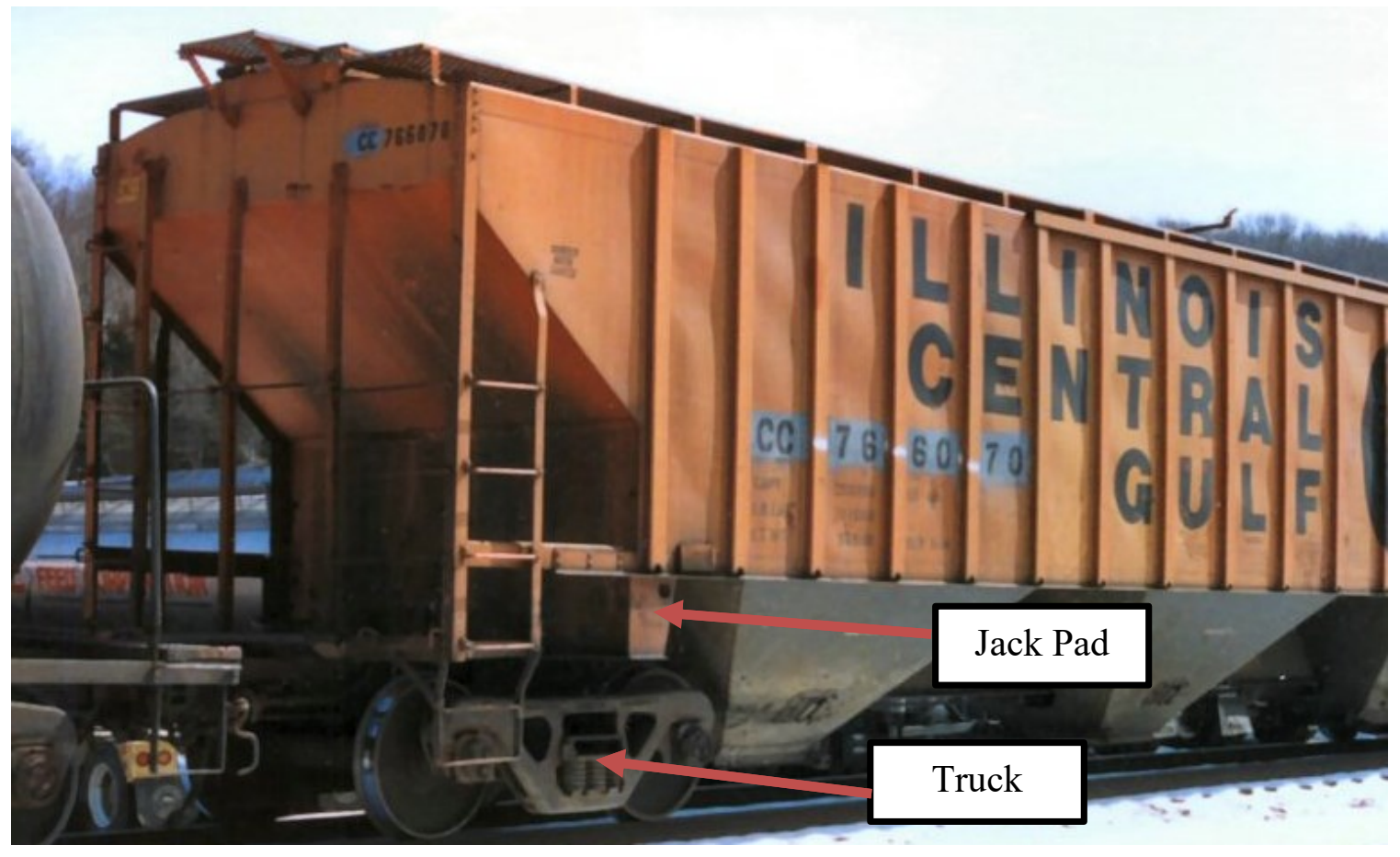

Figure 33: Covered hopper car structure (with side stakes) [31] 


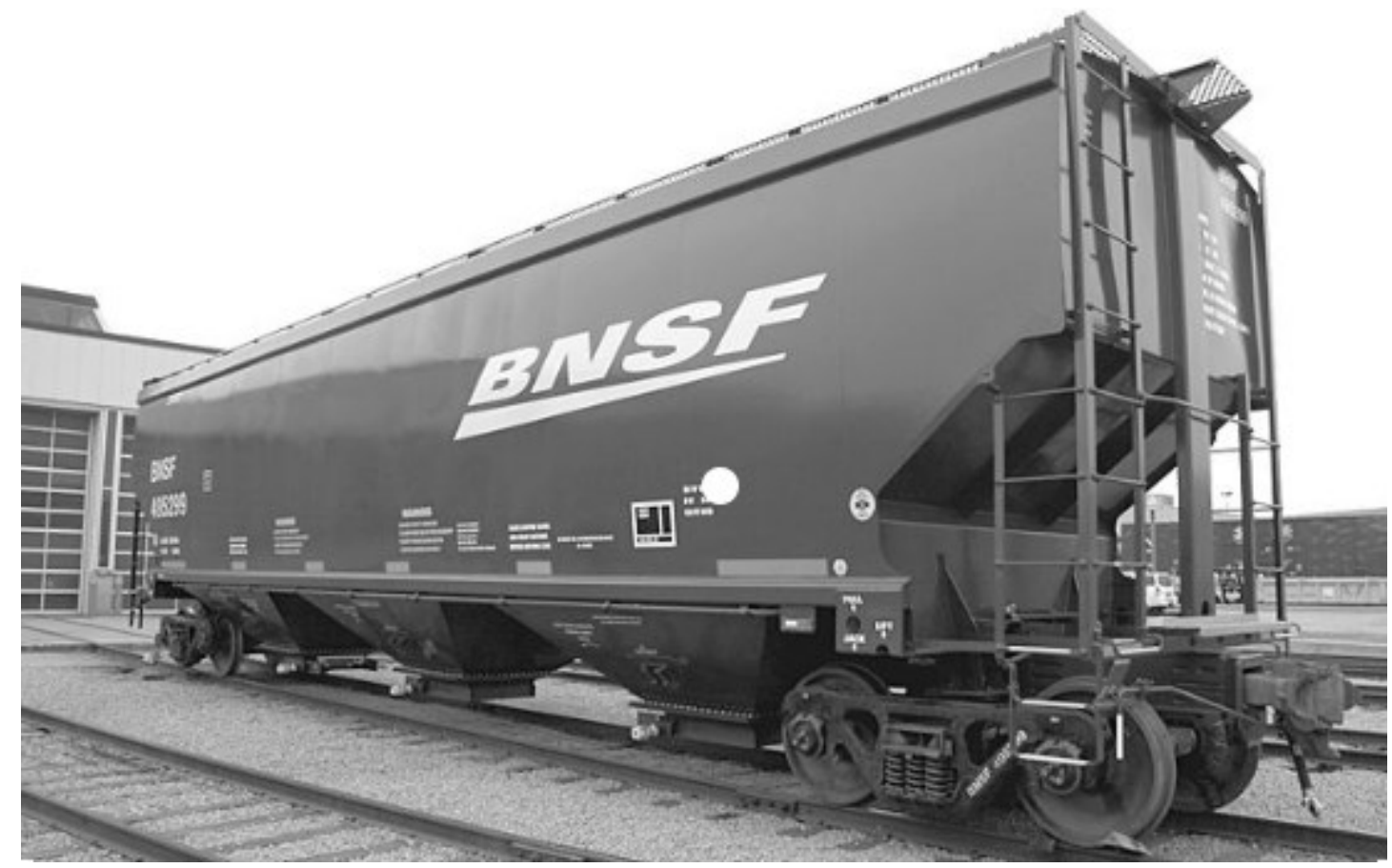

Figure 34: Covered hopper car structure (without side stakes) [31]

"The trucks provide no longitudinal restraint to the car body, and the boundary conditions on support points should not do so either. On a simple whole car model, it is necessary to apply a longitudinal constraint even when there is no longitudinal load or when all longitudinal loads are balanced. As long as this is only applied to a single node, the reaction force will be approximately zero. This constraint could be applied anywhere on the model. When a symmetrical half model is used, either lateral or longitudinal, the symmetrical boundary conditions automatically impose a longitudinal or lateral constraint. So in this case, no further longitudinal or lateral constraint should be applied at any other part of the model" [6].

Here, in order to prevent possible problems arises from applying arbitrary single point constraint, the half model of the car (longitudinal half) was simulated, and symmetry boundary condition was used. The symmetry plane is $\mathrm{x}-\mathrm{z}$ plane, and its normal 
is $\mathrm{y}$ direction. This is generally known as "y-symmetry plane" in ABAQUS, and constrains all out of $x-z$ plane degrees of freedom, i.e. $U_{2}=U_{1}=U_{R}=0$, where $U_{R}, U_{2}$ and $\mathrm{UR}_{3}$ are rotational degrees of freedom around $\mathrm{x}, \mathrm{y}$ and $\mathrm{z}$ axes, respectively. This can be seen in Figures 35 and 36.

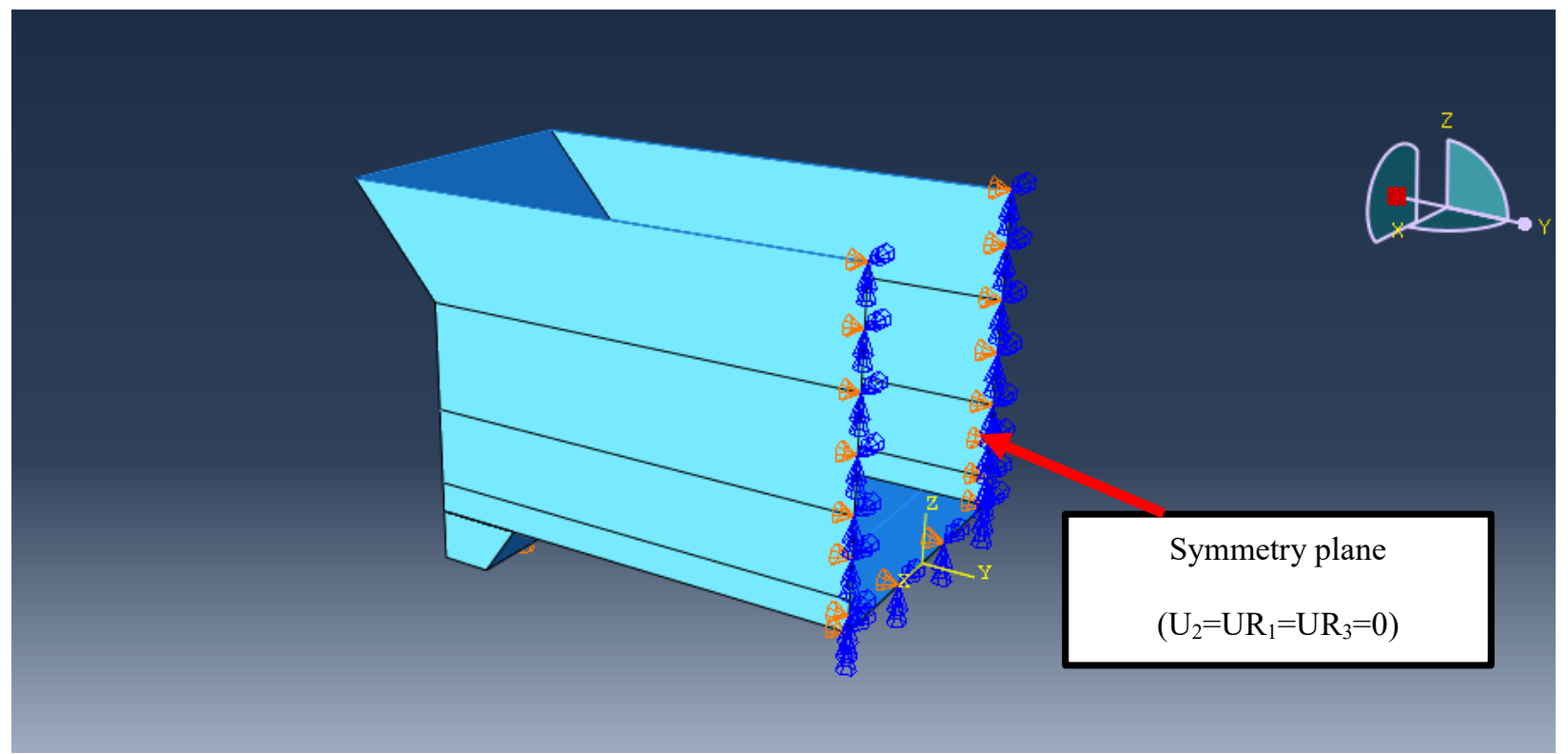

Figure 35: Symmetric boundary conditions

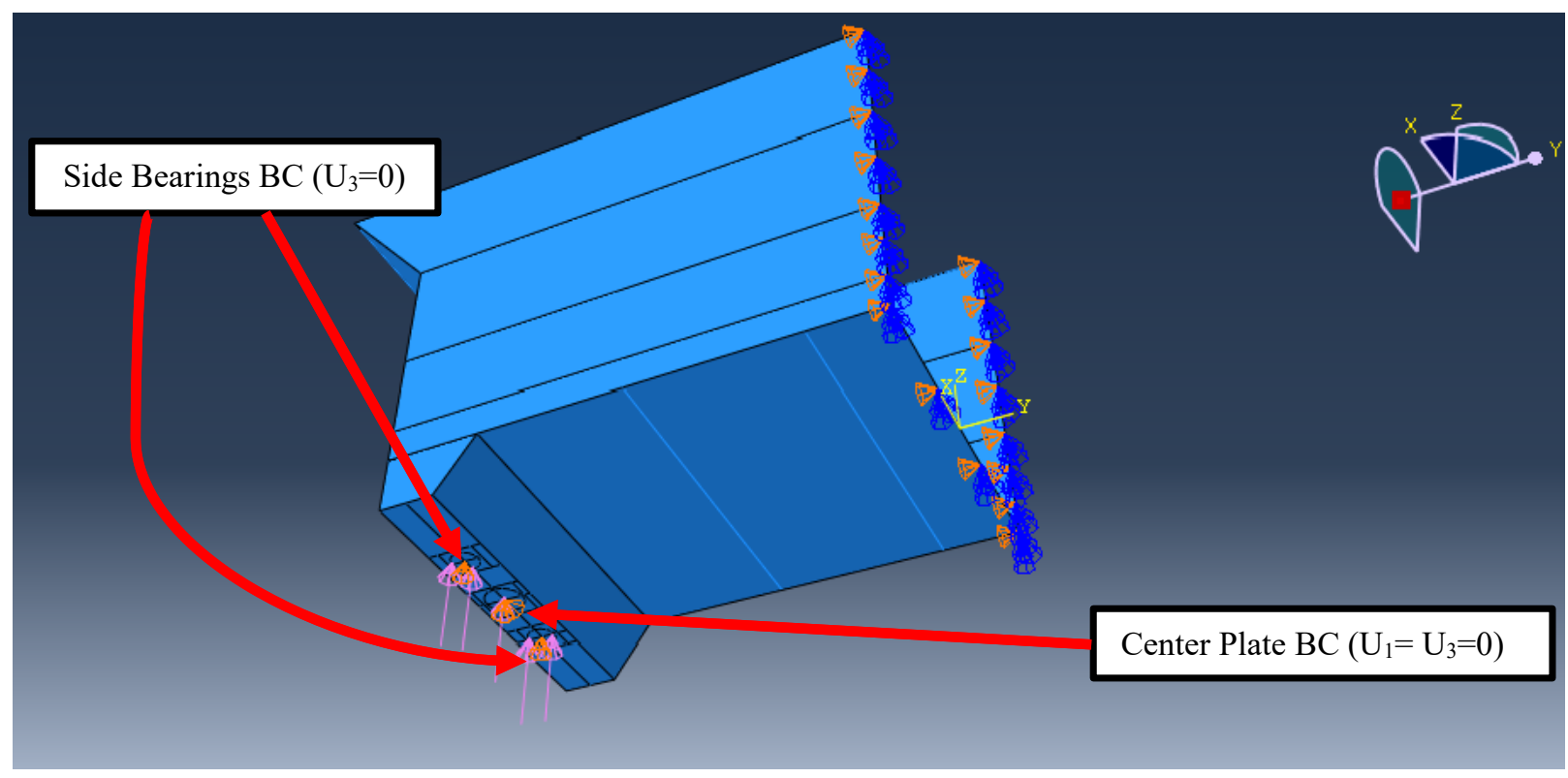

Figure 36: Center plate and side bearings boundary condition with their respective reaction forces 


\subsubsection{Mesh Density}

The accuracy of the FEA results depend highly on the number of elements and nodes (mesh density). Generally, convergence analysis is used to find the suitable number of elements which can fulfill both computational efficiency and accuracy. However, there are some cases where singularities might occur during analysis and is not possible to obtain converged results, i.e. as the mesh density is increased, the stresses will increase almost without limit [6]. This is the case for freight car FE simulation and is predicted in the AAR recommended practice. This happens when two plates are joined using simple plate elements and produce extremely abrupt changes in geometry. These changes can result in mathematical singularities in which the stress concentration factor can be unrealistically large. This is not physically possible, and such stress levels are not appropriate [6].

The stress close to a weld or any discontinuity can be thought of as a free-field stress with stress concentrations applied to it. There is an experimental method for calculating the stress concentration at the weld geometry which will be used for calculating the fatigue life in welds [6] but this is not of concern at present, as the welds are not modeled in this project. However, it is necessary to consider other geometric stress concentrations (GSCs). This might include the geometry of the structure, or other details such as holes, attachments, etc. that may affect the stress. A fair assessment of the free-field stress can be made by taking the stress at specific distance from the weld toe [6]. British Standards [32] recommend taking the stress at about four times the material thickness away from the joint (or from the centerline of the attached plate or the location 
of the FE plate element). This can be established by using elements of this size and taking the stress at the first node from the joint, see Figure 37 [6].

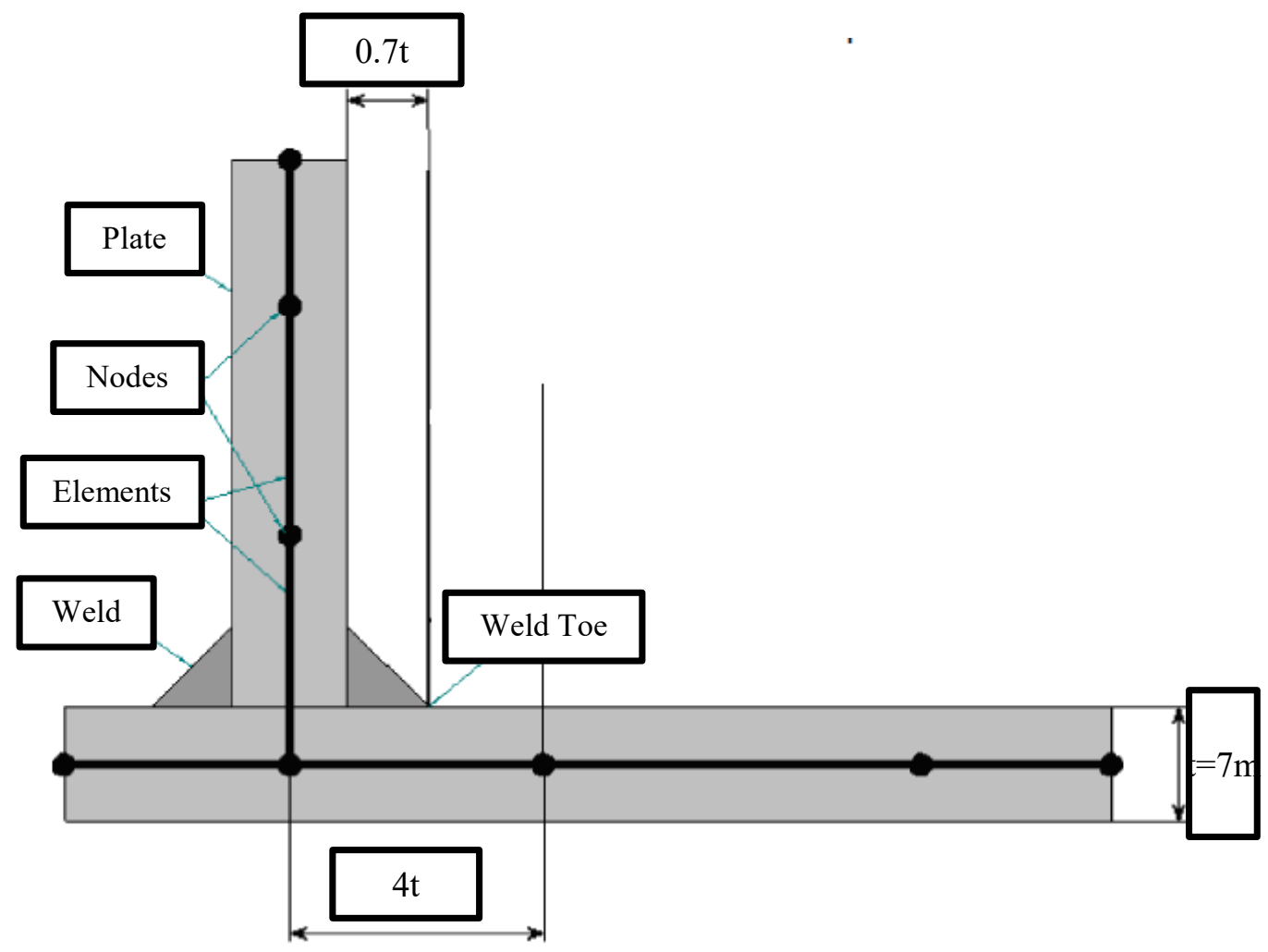

Figure 37: Joint geometry and recommended mesh size [6]

Following British standards [32] results in having $28 \mathrm{~mm}$ size, 4 nodes, quadrilateral shell elements. In addition to size, according to AAR regulations, in regions with high stress magnitudes, shape of quadrilateral elements should be as close to a square as possible. Excessive length-to-width ratio (above 10:1) and angularity (internal angle at corner greater than $155^{\circ}$ ) should not be used [6]. However, the mentioned parameters are only required for regions with high stress magnitudes. Therefore, only the floor panel and its vicinity, are meshed with the stated size and exact square shape. For other regions of the rail car, which have much lower stresses, larger size elements are 
used but the regulations regarding the shape of the element are followed on those sections as well. Conducting mesh verification in ABAQUS shows that all elements on the car body follow those shape restrictions. This proves that even though larger size elements without perfect square shapes are used for low stress regions, their size and shape are still within acceptable range, i.e. aspect ratio is below 3:1 which is even much lower than AAR recommended value, and most of them have $90^{\circ}$ internal angle while none of them exceed $155^{\circ}$.

Again, it must be noted that the supporting structures like side sills, floor panel stiffeners and jack pad are not of the interest for this thesis, shown in Figure 38. However, they have to be modeled for properly predicting the stress distribution on the rail car body. For that, two top side sills (cross section: $90 \mathrm{~mm} \times 90 \mathrm{~mm}$ ), one end short sill (cross section: $90 \mathrm{~mm} \times 90 \mathrm{~mm}$ ), two floor stiffeners (cross section: $120 \mathrm{~mm} \times 120 \mathrm{~mm}$ ) are simulated using linear Timoshenko beam elements (B31), and a jack pad structure is simulated as solid using 3D stress elements (C3D8R) with 8 node linear interpolation, brick shape, reduced integration and hourglass control type element. The choice of Timoshenko beam element over Euler-Bernoulli beam element assures that both thick or slender beam behavior can be accurately simulated [29] so there is no concern about the relation between cross sectional area and length of the beam. The number of elements on mentioned supporting structures are not as important as they are on the rail car body because those supporting structures are not to be studied. In addition, some of them have very low stresses which makes their mesh density even less important. Therefore, larger size elements are used for those structures compared to the freight car body elements. The meshed model can be seen in Figures 39 and 40. 
All the mentioned supporting structures are connected to the body using multi point constraint (tie constraint).

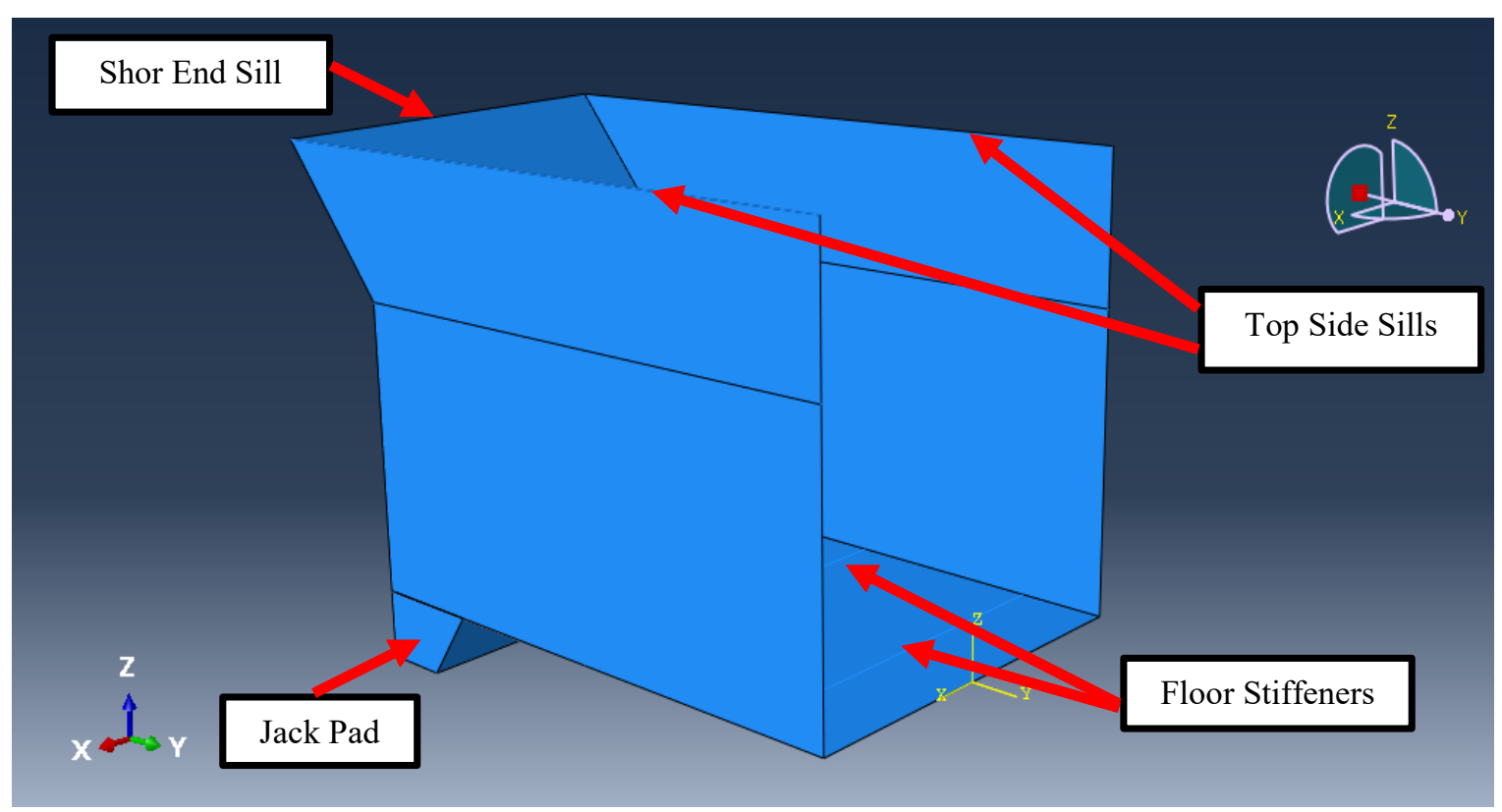

Figure 38: Supporting structures used for simulation

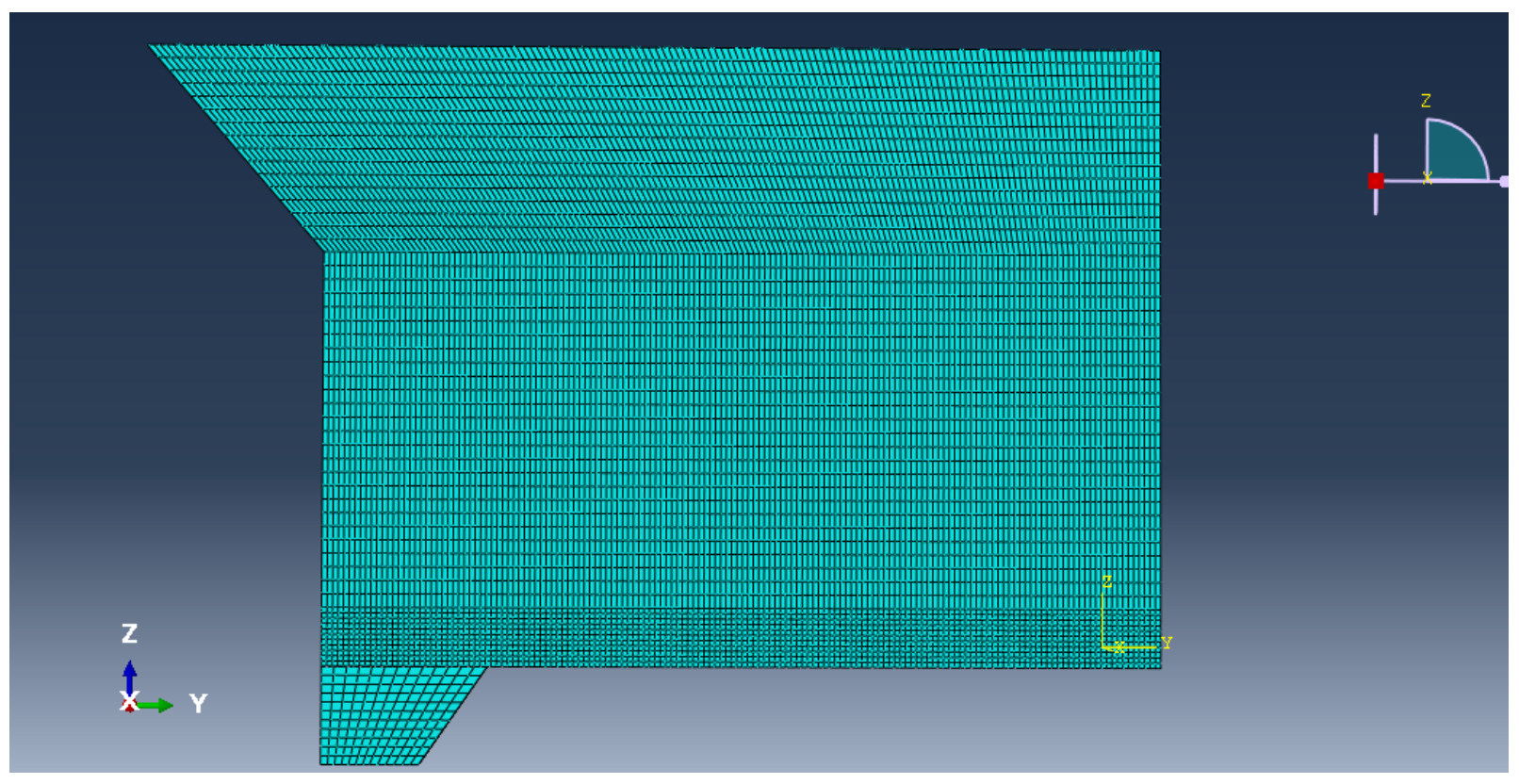

Figure 39: Side view of the meshed model 


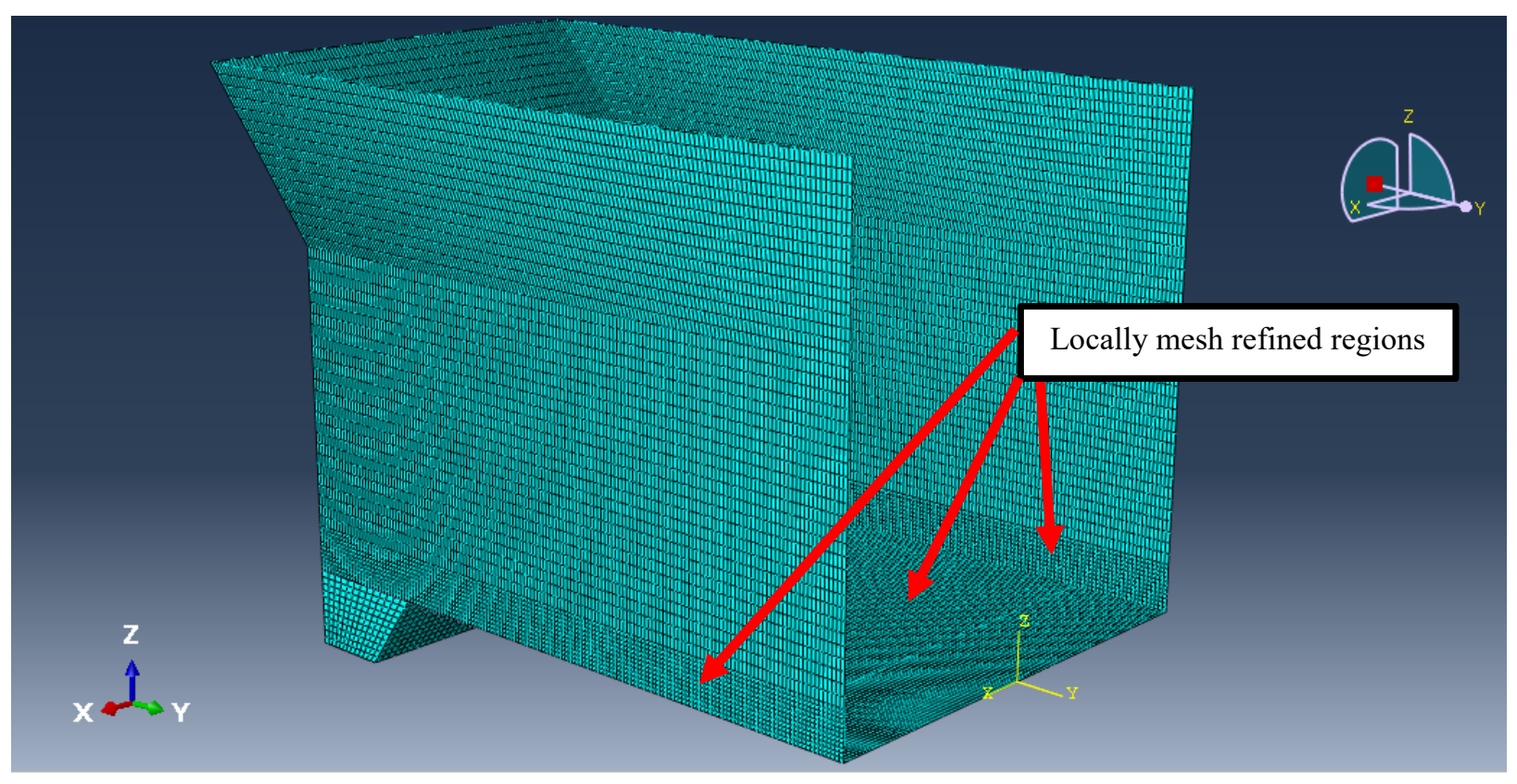

Figure 40: Isometric view of the meshed model

\subsubsection{Result Interpretation}

The equivalent von Mises stress distribution of the selected hopper car body resulted from FE analysis is shown in Figure 41. However, the interpretation of FE results is not straightforward at all. The final stress distribution and its magnitude which is shown by a FEA software may not exactly represent the real behavior of the structure. This can lead to an overweight design or unpredicted failure of the structure. There are some factors involved with this issue and must be considered when interpreting the FEA results for further design steps. Of course, it is not easy to list all those relevant parameters but the ones that are involved with this specific study are explained. 


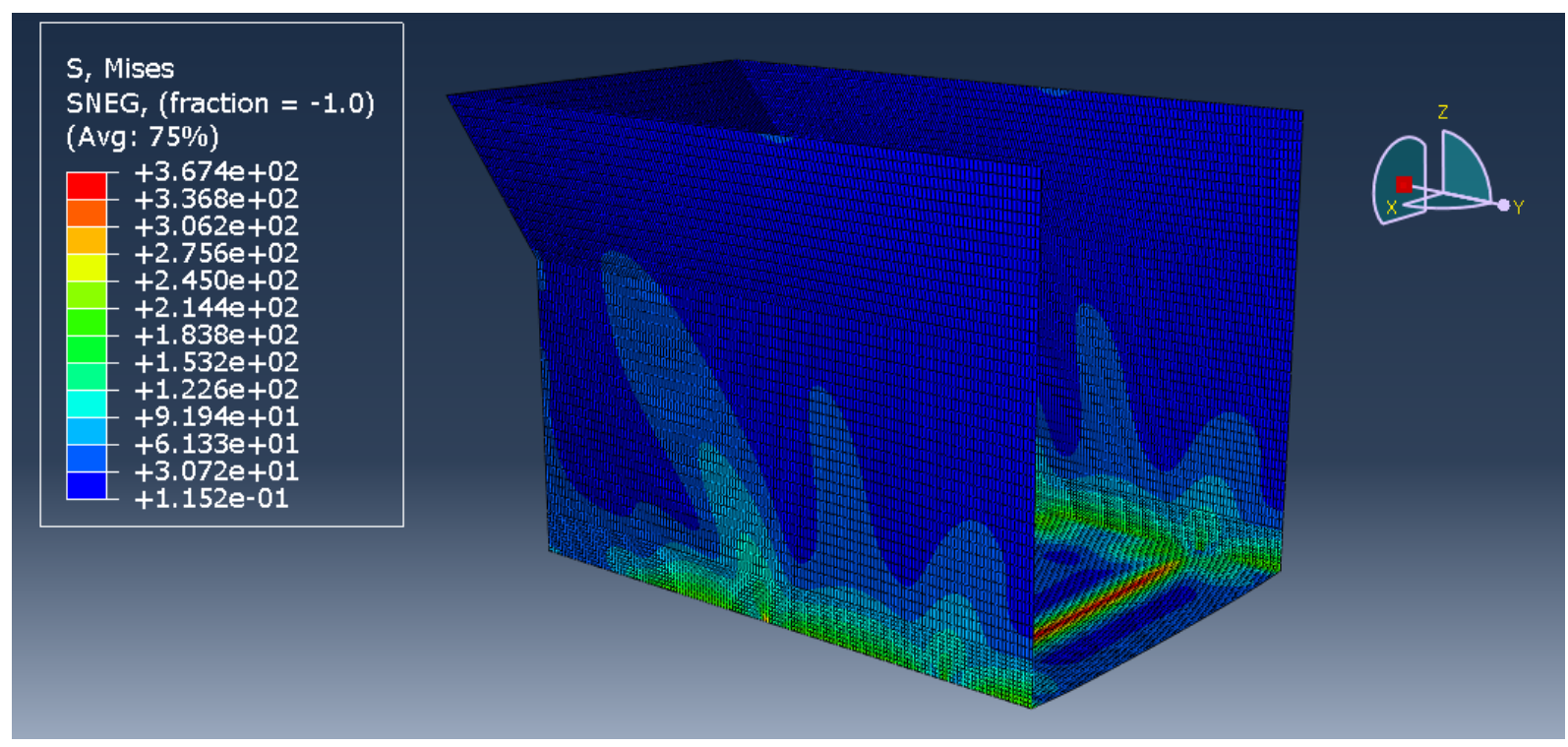

Figure 41: Isometric view of the stress distribution

Nodal averaging: "The calculation of stresses at a node can take into account the stresses in adjacent elements and average these. In situations where the stress in an adjacent element is low due to it not being in the direct load path, this can result in an artificially low level of stress being reported. This is particularly true when stresses are present in a plate and a second plate is welded perpendicularly to it, when this second plate does not take part in the stress field and carries minimal stress" [6].

This might be the case on the edges where side wall is attached to the floor panel. As can be seen in Figure 42, in most parts the resultant stresses at edges are shared between two plates, this mean there is no need to average the stresses on most parts. However, there are some regions, i.e. where floor stiffeners are attached to the edge, that local high stresses appear on one side of the edge, while the other side have very small stresses. These points are the points where nodal averaging is necessary, as one of the sides was put out of the load path. The reason behind this, is explained in element type 
transition. However, if nodal averaging is calculated on this point, the magnitude of the shared stress between elements would be same as other areas of the edge, i.e. labeled as green in the stress contour (183 MPa- $214 \mathrm{MPa})$.

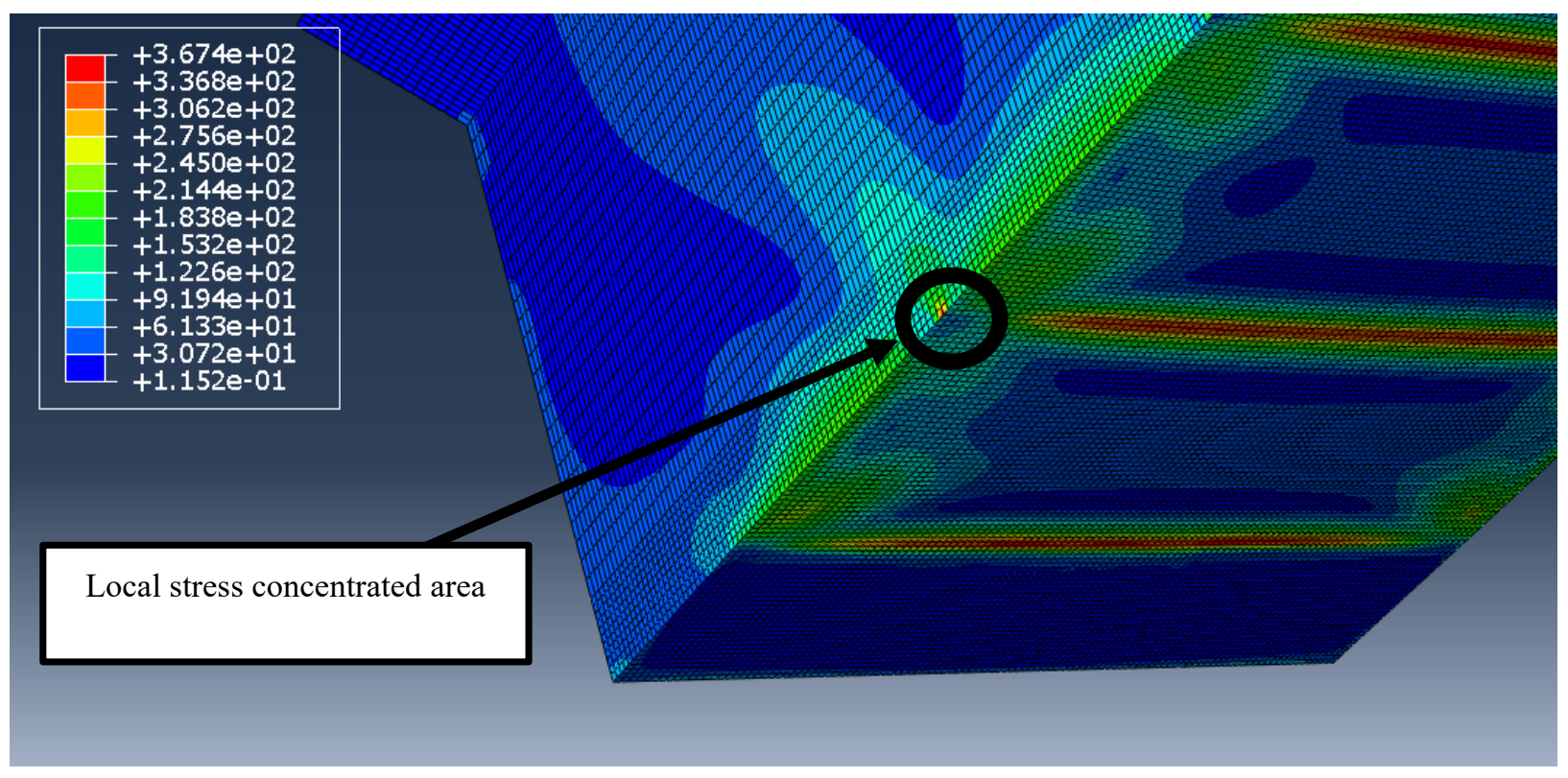

Figure 42: The point that stress is locally concentrated and not shared with neighbor elements

Element type transition: It has to be borne in mind that plate elements do not provide any stiffness at the nodes about an axis normal to the element surface [6], while the nodes of a beam element have this torsional stiffness. Where a beam element shared its node with a shell element which is under torsional loading, the node of the beam element with its different stiffness properties interrupt the typical deformation behavior of a shell element and cause local stress concertation. Apparently, this interruption prevents neighboring shell elements from sharing their stresses. This is the case on edges where side wall shell elements that are close to the edge, are under torsional loading due to the bending of their adjacent elements in the floor panel. That is why the local stress 
concentration on the edges can only be found on points where panel stiffener is attached to the edge. This is less dominant for the stiffener which is closer to the longitudinal symmetric plate (middle of the car body) because as it gets closer to the middle of the car, the slope of the panel defamation (in this case, $\theta=d z / d y$ ) reduces, consequently, the misorientation between adjacent shell elements on side walls and floor panel reduces and this leads to lower torsional load and lower local stresses. These regions can be seen in Figure 43.

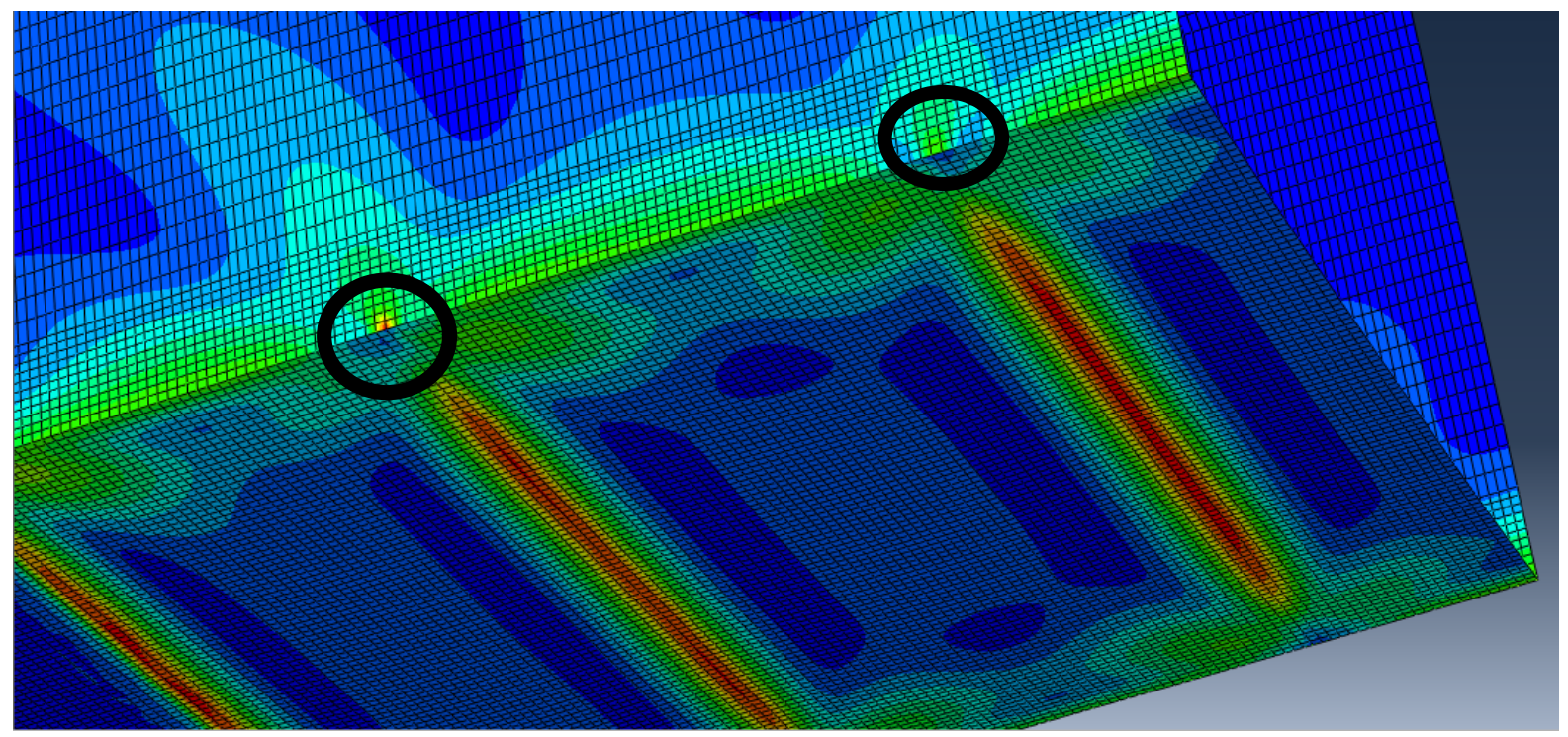

Figure 43: Stress concentration in points where floor stiffeners attached to the edge

Geometrical discontinuity: This was already explained that the stress on any joint, attachment or in general any geometrical discontinuity may not be converged due to singularity, but the effects of those geometrical stress concentration have to be considered [6]. Therefore, as recommended by British standards [32], element size of 4t was used, and the stresses on regions where the floor panel is attached to the jack pad or stiffeners would be considered on the one element away from the attachment point. This is shown 
in Figure 44. By this means, the effect of stress concentration is taken into account without being affected by unlimited stress increase which results from singularity. On regions with highest magnitude of stress, this amount would be in the range of $275 \mathrm{MPa}$ $306 \mathrm{MPa}$.

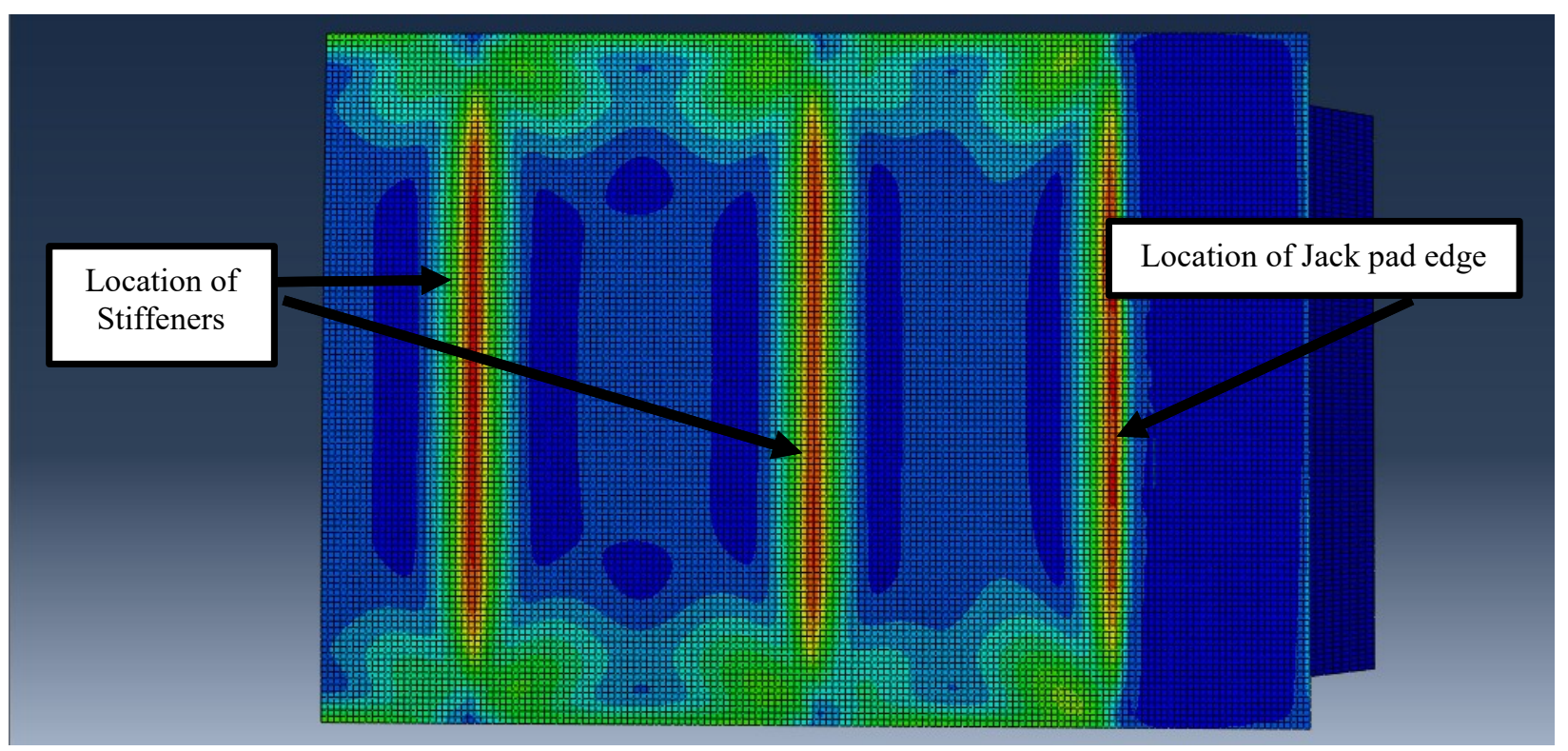

Figure 44: Stress distribution on floor panel

\subsection{Model Preparation Summary}

In this chapter, the CAD model of a covered hopper car was created in CATIA V5 using geometrical data obtained from AAR specifications [6] and the railcar manufacturer. The CAD model was then imported for FE analysis. The type of analysis conducted was linear static stress analysis. In fact, only static loads, i.e. lateral and vertical loads resulted from weight of lading, were considered. However, there are cases that a railcar may pass through a curved path, and that is where the dynamic analysis is required. There are other methods for more realistic simulation of the behavior of the 
cargo in the railcar body. Smooth Particle Hydrodynamics (SPH) and Discrete Element Method (DEM) are known to be the alternative methods for static analysis. By using these methods there would be no need to estimate or simulate the loads as was done for static analysis. Instead, only a collection of points is necessary to represent a given body [29], in this case the cargo. By that mean, it would be also possible to conduct dynamic analysis and take the effects of centrifugal force into account. However, since these methods are not introduced by the AAR Manual and the current thesis is focused only on static loading, these methods will not be used but could be interesting options for future studies.

Another issue that has to be pointed out is the crash (impact) load case. In fact, according to AAR standards, the impact load would mainly be tolerated by under frame structures like bolster or center sill [6]. Of course, there is a vertical load factor called "dynamic load factor" that must be applied to the weight of cargo in the case of impact simulation [6] but the hopper car body is not responsible for resisting the impact load, and it cannot be analyzed unless all corresponding under frame structures are properly modeled and simulated. This would be out of scope for this research.

Another important requirement in freight car structural design is fatigue life prediction. According to AAR standards [6], only the following components and their attachments require fatigue analysis: bolster, center sill and stub center sills, buff and draft attachments and supports, box car doorway area, side sills on center sill-less cars, covered hopper car roofs, covered hopper car interior partitions, flatcar trailer-hitch supports and top chords and top chord reinforcements [6]. In addition to what were mentioned, welds are also considering as critical regions which require fatigue life 
analysis. However, this is not the focus of the research, as components like roof or supporting structures like interior partitions and bolster or even welds are not modeled.

To sum up, in this section a linear static/implicit analysis was conducted to have a general idea about the stress distribution and its magnitude on different regions of the railcar body. The obtained information from this section will be further used for material selection and structural optimization. 


\section{Chapter 3. Material Selection}

\subsection{Introduction}

There is a general procedure for any type of selection in engineering applications, e.g. choice of material, manufacturing method or geometrical shape [33]. However, this chapter is focused on material selection, or more specifically, material substitution.

A typical selection process starts with a definition of the overall kingdom of entities from which the choice is to be made. Figure 45 illustrates how the kingdoms of materials can be subdivided into families, classes, subclasses and members. As an example, the material's kingdom contains the family "Metals" which in turn contains the class "Aluminum alloys", the sub-class "6000 series" and finally the particular material "Alloy 6061". Each member of the materials kingdom, is characterized by a set of attributes include its mechanical, physical, thermal, electrical, optical and chemical properties, its cost and availability and the environmental consequences of its use. In fact, selection is finding the best match between the attributes of the materials in the kingdom and that required by the design [33]. 


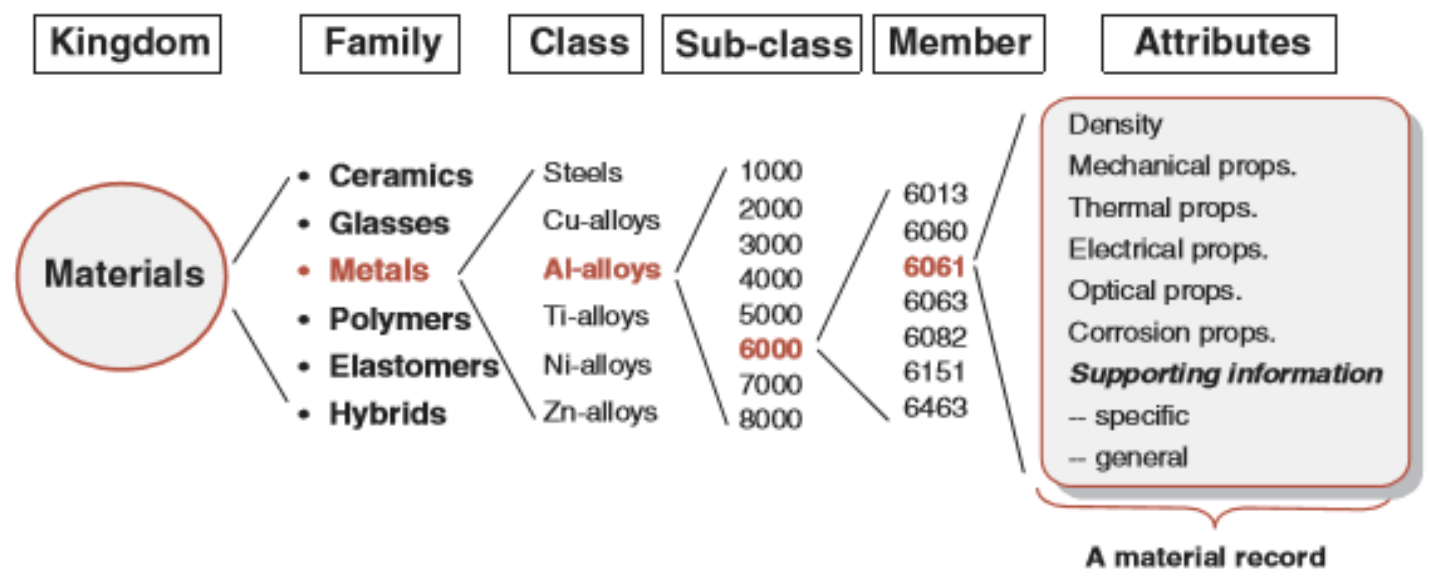

Figure 45: Material kingdom sub-division [33]

\subsection{Selection Strategy}

There are four main steps for material selection: translation, screening, ranking, and supporting information. The explanation of these steps are as follows [33]:

Translation: Reinterpreting the design requirements in terms of function, constraints, objectives and variables.

Screening: Deriving the constraints and applying these to find a subset of viable options.

Ranking: Ordering the viable candidates by the value of a material index, i.e. the criterion of excellence that maximizes or minimizes some measure of performance.

Supporting information: Seeking supporting information like established uses, behavior in relevant environments and availability for the top-ranked candidates to decide about the final choice. 
The top-down process is shown in Figure 46.

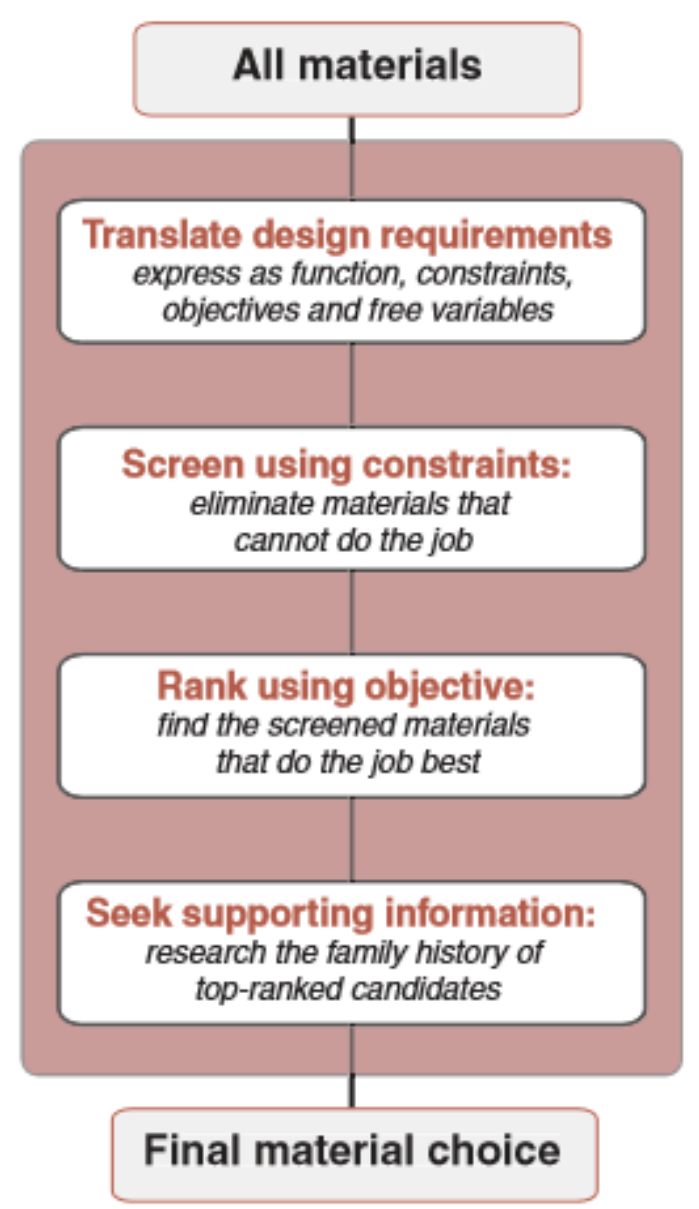

Figure 46: The top-down material selection process [33]

These steps will be further discussed during the material substitution process for a hopper car body. However, before starting the material substitution, it is necessary to study the material indices. In fact, deriving the material indices would be the first step toward material selection/substitution 


\subsection{Material Indices}

Structural elements are components that perform a physical function, e.g. they carry loads, transmit heat, store energy and so on. Indeed, they must satisfy functional requirements that are specified by the design. For example, a tie must carry a specified tensile load, a spring must provide a given restoring force or store a given energy, etc. [33]. The performance of a structural element is determined by three things [33]:

- The functional requirements

- $\quad$ The geometry

- The properties of the material of which it is made

The performance $P^{o}$ of the element is described by an equation of the form [33]:

$$
P^{\mathrm{o}}=\left[\left(\text { Functional }, F^{\mathrm{o}}\right),\left(\text { Geometric }, G^{\mathrm{o}}\right),\left(\text { Material }, M^{\mathrm{o}}\right)\right]
$$

Or

$P^{\mathrm{o}}=f\left(F^{\mathrm{o}}, G^{\mathrm{o}}, M^{\mathrm{o}}\right)$

Where, $P^{\mathrm{o}}$ is the performance metric, and describes some aspect of the performance of the component like its mass, volume, cost, life or force, and " $f$ " means "a function of'. Optimum design is the selection of the material and geometry that maximize or minimize $P^{o}$, according to its desirability or otherwise [33].

The three groups of parameters in the function " $f$ " are said to be separable when the equation can be written [33]: 


$$
P^{\mathrm{o}}=f_{1}\left(F^{\mathrm{o}}\right) \cdot f_{2}\left(G^{\mathrm{o}}\right) \cdot f_{3}\left(M^{\mathrm{o}}\right)
$$

Where $f_{1}, f_{2}$, and $f_{3}$ are separate functions that are simply multiplied together. When the groups are separable, as they frequently are, the optimum choice of material becomes independent of the details of the design, i.e. it is the same for all geometries $\left(G^{\circ}\right)$, and for all values of the function requirement $\left(F^{\circ}\right)$. This enables enormous simplification: the performance for all $F^{\mathrm{o}}$ and $G^{\mathrm{o}}$ is maximized by maximizing $f_{3}\left(M^{\mathrm{o}}\right)$, which is called the material index for short. The remaining, $f_{l}\left(F^{\mathrm{o}}\right)$ and $f_{2}\left(G^{\mathrm{o}}\right)$, is related to the structural index [33].

\subsection{Material Selection for Covered Hopper Railcars}

Material selection is in fact a form of optimization, i.e. optimizing (minimizing or maximizing) the material index (goal function) for the specific application. In this section, the material would be optimized (selected) by optimizing the material index, $f_{3}\left(M^{0}\right)$. This is independent from the structural behavior of the rail car body, i.e. $f_{l}\left(F^{\mathrm{o}}\right)$ and $f_{2}\left(G^{\mathrm{o}}\right)$. Structural optimization will be done separately in the next chapter.

In the following section, those four steps for material selection will be discussed in detail for material substitution in the selected covered hopper car body. This is started with "translation" step and defining the function and constraints. 


\subsubsection{Functional Requirements in Material Selection of Hopper Cars}

In this section, the function, objectives and constraints must be defined. This is shown in Table 6.

Table 6: Parameters for the hopper car material selection [33]

\begin{tabular}{|c|c|c|}
\hline Term & Definition & Application in hopper car \\
\hline Function & "What does the component do?" & Load carrying \\
\hline Objective & "What is to be maximized or minimized?" & Minimizing the weight \\
\hline Constraints & $\begin{array}{l}\text { "What non-negotiable conditions must be } \\
\text { met?" } \\
\text { "What are negotiable but desirable } \\
\text { conditions?" }\end{array}$ & $\begin{array}{l}\text { Any form of structural } \\
\text { instability (yield strength) is } \\
\text { non-negotiable. } \\
\text { The cost (price) is } \\
\text { negotiable. }\end{array}$ \\
\hline
\end{tabular}

As was initially discussed, the target of this thesis is to reduce the weight of current steel railcars to minimize the GHG emission and fuel consumption. Therefore, the objective in material selection is to select a material with the minimum possible weight (density). This means, that the selected material should have lower density compared to the density of the steel which is used in conventional hopper cars. The density of steel usually ranges between $7750 \mathrm{~kg} / \mathrm{m}^{3}$ to $8050 \mathrm{~kg} / \mathrm{m}^{3}$, and thus, the density of the selected material should be smaller than $7750 \mathrm{~kg} / \mathrm{m}^{3}$.

In hopper car structural design, the main function of the body is to carry the specified load of the cargo. As was discussed in the previous chapter, fatigue, impact or even vibration are not of the concern for design of the body. In fact, a hopper car body 
has to carry the weight of lading without any structural instability, i.e. plastic failure or buckling. Thus, the design criterion is the yield strength of the material. In another world, strength is the constraint in selection of the material for a hopper car and is nonnegotiable because the wrong selection of material (e.g. material with low strength) would lead to structural failure, and consequently, the design will be rejected by the AAR.

Cost would also be another constraint in the selection of the best material. Because a new design has to be cost-efficient to be widely used in the industry, especially in railcar industry. In fact, the rail industry is not as competitive as the automotive, or aerospace industries, where those industries invest a higher proportion of funds into research and development projects and new designs. The vendors in rail industry would not use the new technologies unless it is shown to be financially profitable. Therefore, cost would be definitely an important factor, and it is not feasible to select a very light material which is too expensive. However, cost is not as restricted as strength is. This means that the selected material should satisfy at least a minimum value for the yield strength, while there is no pre-defined minimum value for cost, and is negotiable.

It must be noted that the stiffness is not a main constrain in material selection for the hopper car body. Because there are no minimum requirements for deflection of panels as long as the they are within the elastic region. Of course, selecting a material with very low stiffness will lead to large deflections, and is not desirable but stiffness is not a main concern at this step of selection process. This can be later discussed in steps like ranking or supporting information. 
After defining the function, objective and constraints, the material index for this application must be derived. As was already discussed, a typical rail car body is in fact made of different panels. The resultant load from weight of cargo is distributed on these panels (either side or floor panels), and consequently, these panel are under bending. There might be some extent of tensile or compressive loads that appears as a result of the interaction of panels with each other, but these loads are actually internal loads with much smaller magnitudes compared to the dominant bending load. In fact, in many engineering applications, different loads might be applied to the structure but only the dominant loading (or loadings) is being considered for the material selection. In this case, the only external load is the resultant bending load so there is no need to concern about small internal loads.

Consider a case like the loading of a railcar panels. A panel of length $l$, width $b$, thickness $t$ and density $\rho$ is under uniform loading. This is shown in Figure 47. The objective is to minimize the mass $(m)$ of the panel described by [34]:

$m=l b t \rho$

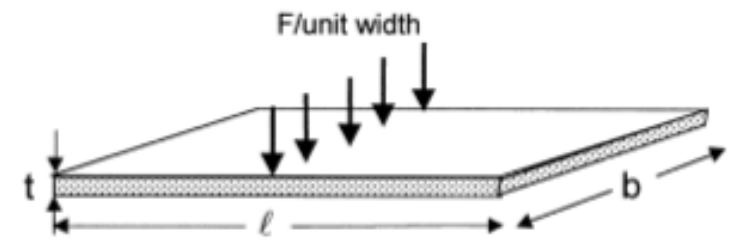

Figure 47: A panel loaded in bending by a force $F$ per unit width [34]

The panel must meet the constraint on its strength, meaning that the applied force should not exceed the critical force [34]: 
$F_{c r} \geq F^{*}$

Where $F_{c r}$ is the critical force per unit length, i.e. the force that its corresponding stress would be equal to the yield strength of the material, and $F^{*}$ is desired force per unit length [34].

To find the critical force, it is necessary to start with stress which results from bending moment.

$\sigma=M c / I$

Where $M$ is the bending moment, $c$ is distance from neutral axis and $I$ is the second moment of inertia of the cross section.

$M=\alpha F b l$

Where $F$ is force per unit length and $\alpha$ is a constant that depends only on the distribution of the load [34]. By substituting the equation (3-7) in (3-6), and $c=t / 2$ :

$\sigma_{\max }=\sigma_{y}=\frac{\alpha F b l t}{2 I}$

$F_{c r}=\frac{K_{0} F b l t}{2 I} \geq F^{*}$

Where $\sigma_{y}$ is the yield strength of the material of which the panel is made. By using equations (3-9) and (3-4), and taking the term $t$ out [34]:

$$
m \geq\left(\frac{6 F^{*} b^{2}}{\alpha}\right)^{1 / 2} l^{3 / 2}\left(\frac{\rho}{\sigma_{y} \frac{1}{2}}\right)
$$


If the above equation is compared with separated form of material performance function (equation 3-3), it is concluded that the material index for this application is $\left(\frac{\rho}{\sigma_{y} \frac{1}{2}}\right)$ [34]. To minimize the mass, this function has to be minimized. This is equal to maximizing the $\left(\frac{\sigma_{y}^{\frac{1}{2}}}{\rho}\right)$, which is the desired material performance metric for this research.

\subsubsection{General Screening Process}

In this section, the materials that are not capable of satisfying the application requirements will be eliminated. This is usually done by using the constraints. According to AAR standards, all materials used for the floor panel should have 50,000 psi (345 MPa) minimum yield strength and a minimum tensile strength of 65,000 psi (450 MPa) [6]. This is well matched with the obtained results in Chapter 2, where the highest von Mises stress on the floor panel ranged $275 \mathrm{MPa}(39,885 \mathrm{psi})$ to $306 \mathrm{MPa}(44,381 \mathrm{psi})$. This is almost $15 \%$ below the recommended minimum yield strength and can be considered as the margin of safety in selection of new materials for the side panels as well.

The stated requirement was specifically for the floor panel. However, there is also a general instruction for a railcar design that can be used for the material selection of side walls. According to the AAR Manual, "the allowable design stress shall be the yield or $80 \%$ of ultimate, whichever is lower, or the critical buckling stress" [6]. In fact, the yield strength or $80 \%$ of the ultimate strength of the chosen material, whichever is smaller, must be higher than the maximum von Mises stresses that were derived in the previous chapter. From Chapter 2, the maximum von Mises stress on the side walls (their 
bottom) was $214 \mathrm{MPa}$. Therefore, the selected material should have at least a yield strength higher than $214 \mathrm{MPa}$. However, from a conservative design perspective, it is better to consider a margin of safety (almost 15\%) for the selection of material, i.e. only the materials with yield strength higher than $250 \mathrm{MPa}$ and ultimate strength higher than $310 \mathrm{MPa}$ are being considered for the side walls. By that mean, it is assured that the selected material would not fail under any extreme cases, and any possible computational errors, which are inherent for the FE simulation such as errors caused from the singularity and reading the stresses on one element away from the joints, are considered.

Another constraint that can be set is about the family of materials, e.g. metallic, ceramic, etc. This is necessary to narrow the feasible set for selecting the material because otherwise, the selection would be confusing. This constraint is defined based on the current industrial trends and the thesis targets.

In the first chapter, three different alternatives for conventional steel railcars were discussed, Korean Tilting Train project [9], i.e. full composite body, hybrid steelcomposite body and full aluminum body. Full composite body was introduced as the ideal case, while full aluminum body introduced as a potential alternative to the full composite scenario [9]. However, in another publication [11] it was concluded that for bus/truck application (long distance applications), aluminum is a better option than the composite scenario in terms of energy saving and recyclability. In addition, even in Korean train project [9] the material scenario named as full composite, was not actually fully made of composite. In fact, it was a sandwich structure which was made of aluminum honeycomb core and carbon fiber epoxy layers. Therefore, it is difficult to say 
whether the aluminum or composite scenario would be the ideal case for weight reduction.

Nevertheless, the present research cannot only rely on previous researches, and intention is to evaluate material and structural options in a scientific manner. However, it is still necessary to limit the feasible space for material selection. Apparently, the current alternatives are composite materials and aluminum (from metallic family) but this project is only focused on selecting a material from the metallic family. There are some reasons to support this idea.

First, the similarity of the metallic type bodies to steel type in the simulation process. It is known that the material properties of the steel are more like other metallic materials as compared to composites. In fact, due to anisotropic behavior of composite materials, it would be more complicated to simulate them. Second, design constraints must be set based on AAR standards [6], while there are no specific standards for composite railcar bodies because they are not still widely used in the North American rail industry. Third, the industrial trend, especially for cargo railcars in North America, is not toward using the composites.

As was already explained, the rail industry is way behind from aerospace or automotive industry in using novel technologies. This case is even worse when it comes to freight type railcars. The freight car operators/producers in Canada, still frequently use steel type cars. They do not tend to replace their heavy old-design cars even with lighter metallic materials, unless the Governmental regulations force them to do so. Even in that case, expecting them to rapidly shift from steel type cars to a composite type, with its associated challenges in design and manufacturing, would be far from mind. Finally, as 
was explained, there are still arguments regarding weather the aluminum scenario or composite one is the most environmental friendly case for this type of application. This means that not only it is still not assured if the composites have advantages over the aluminum, considering their whole life cycle including manufacturing, recycling, etc. but also it was concluded that for long distance applications, aluminum can be a better choice. This is even more likely the case for freight cars in Canada, where they have to travel long distances from east to west and vice versa, in contrast to passenger cars that travel shorter distances.

Based on these considerations, it was decided that the metallic family would be a better option for choosing a new material for this research. In fact, it is better to develop the technology gradually, i.e. smoothly moving from steel cars to other lighter metallic options instead of skipping this step and jumping directly to composite railcars.

\subsubsection{Screening Using CES Software}

So far, the material index, constraints and material family were defined. However, as the kingdom of material, even its metallic family, is so broad, it is not possible to manually select the material without possibly missing some of the options. Thus, the Cambridge Engineering Selector (CES) Software was used for implementing the constraints and material index to find the best candidates. In fact, the CES system was designed to implement the selection principles described above [33]. Before start to use the software, it is better to summarize the objectives and constraints. The related information is listed in Table 7. 
Table 7: Material selection criterion

\begin{tabular}{|c|c|c|c|}
\hline $\begin{array}{c}\text { Target } \\
\text { Structure }\end{array}$ & $\begin{array}{c}\text { Non- Negotiable } \\
\text { Constraints }\end{array}$ & Negotiable Constraints & $\begin{array}{c}\text { Material } \\
\text { Index }\end{array}$ \\
\hline Side Panels & $\begin{array}{c}\sigma_{y} \geq 250 \mathrm{MPa} \\
\sigma_{u l t} \geq 310 \mathrm{MPa} \\
\rho \leq 7750 \mathrm{Kg} / \mathrm{m}^{3}\end{array}$ & $\begin{array}{c}\text { Cost } \\
\text { (Not much more } \\
\text { expensive than steel) }\end{array}$ & $\operatorname{Max}\left(\frac{\sigma_{y^{\frac{1}{2}}}}{\rho}\right)$ \\
\hline Floor Panel & $\begin{array}{c}\sigma_{y} \geq 345 \mathrm{MPa} \\
\sigma_{u l t} \geq 450 \mathrm{MPa} \\
\rho \leq 7750 \mathrm{Kg} / \mathrm{m}^{3}\end{array}$ & $\begin{array}{c}\text { Cost } \\
\text { (Not much more } \\
\text { expensive than steel) }\end{array}$ & $\operatorname{Max}\left(\frac{\sigma_{y^{\frac{1}{2}}}}{\rho}\right)$ \\
\hline
\end{tabular}

As is listed above, the material index is $\left(\frac{\sigma_{y}^{\frac{1}{2}}}{\rho}\right)$, and thus, the required graph would be " $\sigma_{y}-\rho$ ". The resulting graph and suggested materials are shown in Figure 48. The graph has a logarithmic scale, and the dotted lines are the material index with different starting points. That is:

$\frac{\sigma_{y}^{1 / 2}}{\rho}=C$

$\log \left(\sigma_{y}\right)=2 \log (\rho)+2 \log (C)$

The above equation, is a family of straight parallel lines with a slope of "2" on a plot of $\log \left(\sigma_{y}\right)$ against $\log (\rho)$ each line corresponds to a value of the constant $C$ [33]. 


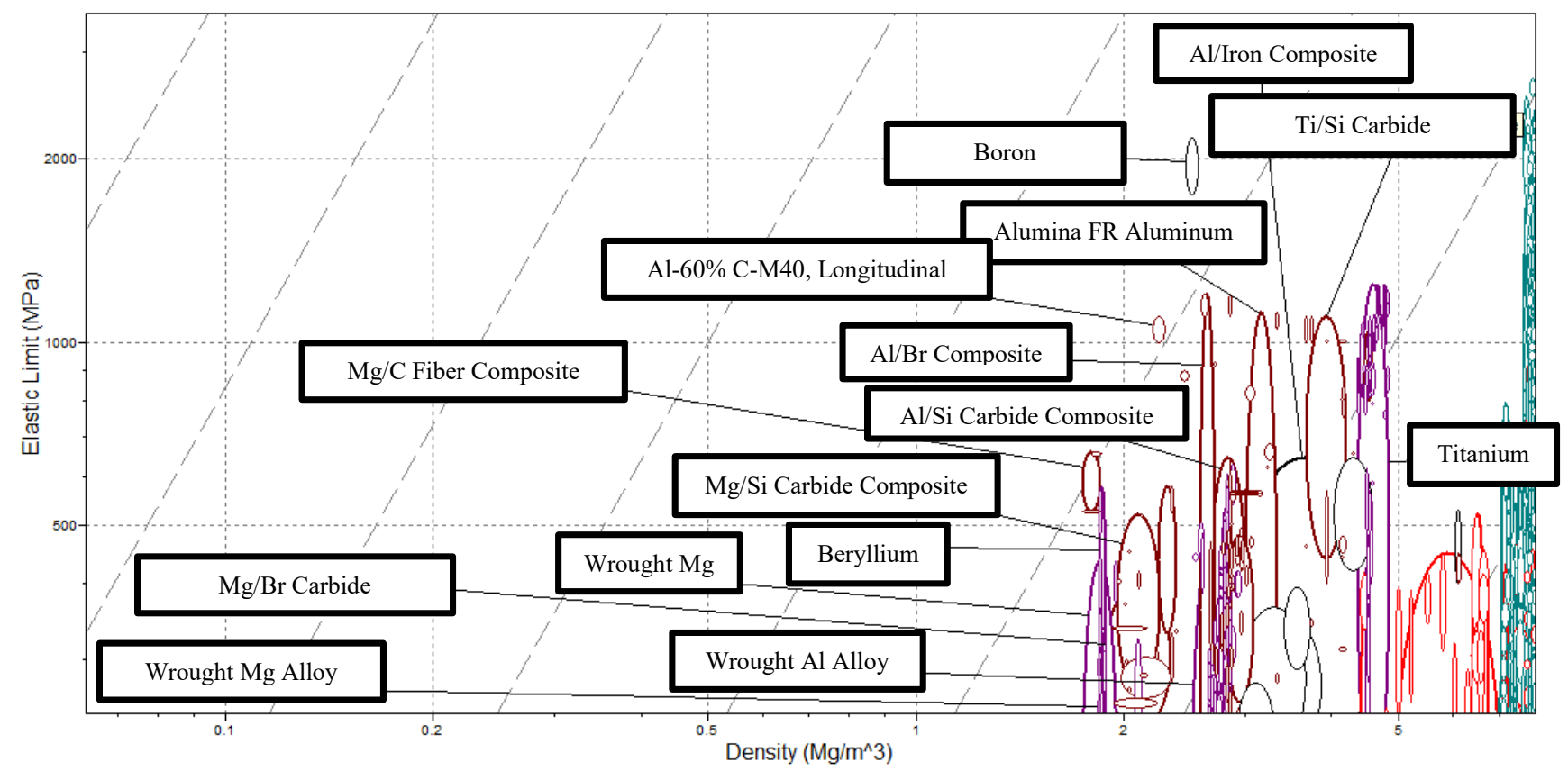

Figure 48: The CES generated graph for material selection

The constraints for side wall panels are implemented in this graph, i.e. the maximum density is almost $7500 \mathrm{~kg} / \mathrm{m}^{3}\left(7.5 \mathrm{Mg} / \mathrm{m}^{3}\right)$ and the minimum yield strength is $250 \mathrm{MPa}$. So all materials shown in the graph are within feasible set. However, as was previously discussed, the best result would be achieved by maximizing the material index. This would be on top left side of the graph, where both low density and high strength are satisfied. In contrast, bottom right side of the graph, represents the worst results. There is no real material on the top left side of the graph. Consequently, the candidate materials must be selected somewhere in between the desired and non-desired regions, preferably as close as possible to the top left side. For that reason, all highdensity materials on the right side (marked with red bubbles and green bubbles) would be eliminated, and only the materials marked with purple, black and brown bubbles are considered. Almost all these materials are labeled in Figure 48. 


\subsubsection{Ranking and Supporting Information}

Although most of the mentioned materials can be regarded as candidates for further study, it was already discussed that the focus would be only on metallic materials and not any type of metal or polymer composite. This means that the only candidate materials are: wrought aluminum alloys, wrought magnesium alloys, wrought magnesium, beryllium, boron and titanium. However, it is known that boron, beryllium and titanium are expensive metals so despite their good mechanical properties, they would not be financially justifiable for a freight rail application. As a result, the final competition will be among wrought aluminum alloys, wrought magnesium alloys and wrought magnesium. The related data for these materials is listed in Table 8.

Table 8: Mechanical and thermal properties of the candidate materials $[35,36]$

\begin{tabular}{|c|c|c|c|}
\hline Type of Material & $\begin{array}{c}\text { Wrought } \\
\text { Aluminum } \\
\text { Alloy }\end{array}$ & $\begin{array}{c}\text { Wrought } \\
\text { Magnesium }\end{array}$ & $\begin{array}{c}\text { Wrought } \\
\text { Magnesium } \\
\text { Alloy }\end{array}$ \\
(ZK60A-F)
\end{tabular}


It must be noted that prices are continuously changing due to different reasons like demand, availability, etc. The listed prices in Table 8 , are the average global prices in March 2018, and are subject to change. For comparison purposes, it worth noting that the global steel price in March 2018 was about 0.42 USD/kg [37].

As can be seen, the density of wrought magnesium and its alloys are almost one third of aluminum alloys, while their price is not much higher. However, despite their good mechanical properties, no rail manufacturer has ever used magnesium alloys in railcar structures. This is due to their high flammability and the risk of fire. In fact, magnesium can be easily ignited when in contact with oxygen, and once the fire started, it can easily spread all over the specimen. This is an important issue because sometimes freight cars carry coal or chemicals, which are themselves flammable. Thus, magnesium and its alloys will be eliminated from the selection process.

The restrictions on the usage of magnesium alloys are not only limited to rail industry. In fact, although the primary use of magnesium is in aerospace and automotive industry, for several decades there were restrictions on magnesium use in aircraft interiors, in particular the passenger cabin [38]. Although no explicit justification was found, it is believed that the primary concern is the flammability and risk to passenger safety. In addition to this, relatively low corrosion resistance of magnesium in aqueous solutions also hindered its non-cabin aerospace applications. Recently, in response to the need of reducing aircraft weight, Federal Aviation Administration (FAA) and various aerospace specification committees are changing their strict regulations on magnesium usage in aircraft interiors [38]. It has been discovered that alloying of magnesium with rare earth metals and other elements having a high affinity to oxygen can lead to the 
ignition-resistant and non-flammable magnesium alloys. However, because of the high cost and availability issues of the rare earth metals, non-flammable magnesium alloys will face economic barriers preventing their wide-scale industrial application [38].

As was discussed above, magnesium alloys cannot be good options for the railcar body structure. Of course, they can be the potential alternatives for future with the development in metallurgy and material science but currently, they cannot compete with aluminum. However, the aluminum mechanical properties that are stated in Table 8, cover a very wide range. That is because of the large number of members in the aluminum alloys' class. In fact, they ranged from weak alloys with $30 \mathrm{MPa}$ yield strength to very strong ones with $510 \mathrm{MPa}$ yield strength. Thus, the final step is to pick the best choice among aluminum alloys. This can be done again by using CES Software, as it lists all aluminum alloys that exist in the bubble corresponding to the aluminum alloy class. The final selection process cannot be done by just using the software. In the last step, it is necessary to gather additional material data from other sources, and some engineering judgments must be applied to make the final selection.

\subsection{Aluminum Application in Railcars}

At this final stage, the selection is not as straight forward as it was in previous stages. Since the remaining materials (aluminum alloys) have already passed several filtering steps, and they all have their own advantages which makes it difficult to just

eliminate one because of not satisfying the constraints. In fact, the simple "pass" or "reject" procedure is not applicable here. For the proper selection, it is necessary to at 
least generally review different aluminum alloy sub-classes. For wrought alloys a fourdigit system is used to produce a list of wrought composition families as follows:

- 1xxx: Controlled unalloyed (pure) composition, used primarily in the electrical and chemical industries [39].

- 2xxx: Alloys in which copper is the principal alloying element, although other elements, notably magnesium, may be specified. 2 xxx series alloys are widely used in aircraft where their high strength (yield strengths as high as $455 \mathrm{MPa}$ ) is valued [39]. This series is heat-treatable and mainly used in aircraft engine parts, rivets and screw products. Most 2xxx series alloys are considered poor for arc welding because of their sensitivity to hot cracking. These alloys are generally welded with 4043 or 4145 series filler electrodes, which have low melting points to reduce the probability of hot cracking [40].

- 3xxx: Alloys in which manganese is the principal alloying element, used as general-purpose alloys for architectural applications and various products [39]. It is improved through strain hardening to provide good ductility and improved corrosion properties. The $3 \mathrm{xxx}$ series is excellent for welding and not prone to hot cracking but its moderate strengths do prevent this series from being used in structural applications [40].

- 4xxx: Alloys in which silicon is the principal alloying element, used in welding rods and brazing sheet [39]. The silicon reduces the melting point of aluminum and improves fluidity. Its principle use is as filler metal [40]. 
- 5xxx: Alloys in which magnesium is the principal alloying element, used in boat hulls, gangplanks, and other products exposed to marine environments [39]. The addition of magnesium brings excellent weldability with a minimal loss of strength and is basically not prone to hot cracking. In fact, the $5 \mathrm{xxx}$ series has the highest strength of the non heat-treatable aluminum alloys. It is used for chemical storage tanks and pressure vessels at elevated temperatures as well as structural applications, railway cars, dump trucks and bridges because of its corrosion resistance [40].

- 6xxx: Alloys in which magnesium and silicon are the principal alloying elements, these alloying elements are combined to present this medium-strength, heattreatable series. It is principally used in automotive, pipe, railings, structural and extruding applications [39]. The $6 \mathrm{xxx}$ series is somewhat prone to hot cracking, but this problem can be overcome by the correct choice of joint and filler metal. This series can be welded with either $5 \times x x$ or $4 \times x x$ series without cracking - adequate dilution of the base alloys with selected filler alloy is essential [40].

- 7xxx: Alloys in which zinc is the principal alloying element (although other elements, such as copper, magnesium, chromium, and zirconium, may be specified), used in aircraft structural components and other high-strength applications. The 7xxx series are the strongest aluminum alloys, with yield strengths higher than $500 \mathrm{MPa}$ possible [39]. The weldability of the $7 \mathrm{xxx}$ series is compromised in higher copper grades, as many of these grades are crack sensitive. Grades 7005 and 7039 are weldable with 5xxx fillers [40]. 
- 8xxx: Alloys characterizing miscellaneous compositions. The 8xxx series alloys may contain appreciable amounts of tin, lithium, and/or iron [39]. Most of these alloys are not commonly welded, though they offer very good rigidity and are principally used in the aerospace industry. Filler metal selection for these heat-treatable alloys include the $4 \times x x$ series [40].

- 9xxx: Reserved for future use [39].

Aluminum alloys series $1 \mathrm{xxx}, 3 \mathrm{xxx}$ and $4 \mathrm{xxx}$ do not have high performance structural application because of their low or medium strength levels. In fact, the choice of aluminum alloy for rail industry is based on ease of fabrication, mechanical strength, weldability and corrosion resistance [41]. Series $5 \mathrm{xxx}$ and $6 \mathrm{xxx}$ have been traditionally used in the rail industry because of their desired mechanical properties such as good weldability and corrosion resistance. Series $6 \mathrm{xxx}$ can offer higher strength and good extrudability as well. According to the Aluminum Association (AA) manual on the repair of aluminum railcars, the commonly used sheet or plate materials are: alloys 5052, 5083, 5086,5454 , and 6061 . The commonly used extrusion materials are 5083, 6061, and to a lesser degree, 7005. Newer alloys, such as 5059, 5383, and 6082, have also been used for railcar applications [41].

In the past, there were challenges with weldability of high strength $7 \mathrm{xxx}$ and $2 \mathrm{xxx}$ series alloys using conventional high temperature welding methods, like arc welding, but the development of Friction Stir Welding (FSW) has changed the situation. While this method has been used for different metals, its application for aluminum alloys is the most dominant. This is due to the fact that tooling costs are lower for aluminum, since its 
melting temperature is relatively low $\left(\sim 660^{\circ} \mathrm{C}\right)$ as compared to steel $\left(\sim 1500^{\circ} \mathrm{C}\right)$, for example. This ensures that the wear of the of the spinning tool and required forces for welding process are much lower. FSW is an ideal process for welding of the longitudinal extruded sections, which is the case in roof and floor panels of the passenger or freight railcars [41]. "A study at the South Dakota School of Mines \& Technology indicated that cost savings of greater than $20 \%$ could be obtained with FSW as compared to the baseline bolted design when fabricating aluminum hopper cars" [41].

There are some reasons that can convince the railcar producers to shift from conventional $5 \mathrm{xxx}$ and $6 \mathrm{xxx}$ series to high strength $2 \mathrm{xxx}$ or $7 \mathrm{xxx}$ series. First, using high strength aluminum alloys can lead to more weight reduction in structural optimization, better payload capacity and more fuel saving. Moreover, the FSW technology associated with these type of alloys, was shown to be cost-efficient (compared to arc welding) and a source of weight reduction itself (compared to using bolts or rivets). Finally, it has to be reminded that the arc welding is also possible for some of these alloys, i.e. 7xxx and $2 \mathrm{xxx}$. The only point is that more attention should be paid for selecting the compatible filler material to prevent post-weld cracking, for example grades 7005 and 7039 are weldable with 5xxx fillers. Consequently, instead of studying the conventional aluminum alloys, the research will be focused on alloys that have not been widely used in the rail industry. In another words, the focus will be on the materials that are used in the aerospace industry because often the materials development in the rail industry follows the trend in the aerospace industry as has been seen in the adaption of composites in passenger railcar applications. Some of the candidate alloys are listed in Table 9. 
Table 9: Mechanical properties of selected wrought aluminum alloys $[42,43]$

\begin{tabular}{|c|c|c|c|c|c|c|c|}
\hline Alloy class & Al 8090 & Al 7075 & Al 7039 & Al 7005 & Al 2014 & Al 2219 & Al 2024 \\
& (T6511) & (T6) & & (T6) & (T6) & (T87) & (T3) \\
\hline Density (Kg/m $\left.\mathbf{m}^{3}\right)$ & 2540 & 2810 & 2740 & 2780 & 2800 & 2840 & 2780 \\
\hline Elastic limit (MPa) & 370 & 503 & 380 & 310 & 414 & 393 & 345 \\
\hline Tensile Strength (MPa) & 450 & 572 & 450 & 380 & 483 & 476 & 483 \\
\hline Elongation (\%) & 7 & 11 & 13 & 12 & 13 & 10 & 18 \\
\hline Young's Modulus (GPa) & 77 & 71.7 & 69.6 & 70 & 72.4 & 73.1 & 73 \\
\hline Shear Modulus (GPa) & 25 & 26.9 & 26 & 26 & 28 & 27 & 28 \\
\hline Poisson's Ratio & 0.33 & 0.33 & 0.33 & 0.33 & 0.33 & 0.33 & 0.33 \\
\hline
\end{tabular}

\subsubsection{Challenges with $2 \times x x$ and $7 \times x \times$ Series Aluminum Alloys for Rail}

\section{Applications}

Alloys 2xxx and 7xxx series, almost have similar properties, i.e. they all have high strength, are heat-treatable and have some issues with arc welding. But as was discussed, the weldability issue can be solved either by using friction stir welding or selecting the proper filler material for arc fusion welding. Because of their similar performance, it is not easy to simply select one of them, but the low density and high strength can be the basis of selection. According to the mentioned basis, alloy 7005 will be eliminated due its lowest strength. Another issue which needs an extra care is that no matter what filler material is being used, alloys 2024 and 7075 are very sensitive to crack 
in case of arc welding. In fact, the only practical way is to use friction welding, and this limits the fabrication options of the manufacturer in case of using these two alloys. Nevertheless, it has been reported that FSW, compared to arc welding, achieves high weld strength, good weld quality and low cost [44]. Even in some cases, if proper care is taken, weld properties become equal to those of the base material [45]. Thus, same as materials, following the fabrication trends in aerospace industry, it is recommended by the author to use FSW instead of arc welding to achieve better quality, save cost and easily select materials with high strength. In fact, this has been brought into practical use in business jets where the fuselage has a seam welded structure for which FSW is used. A construction technique has also been developed in which ribbed extruded parts are parallelly welded with FSW for significant cost benefits [44]. As an example, high speed aluminum railcars such as the Japanese Shinkansen are normally built from complex double skin extrusions in 6xxx alloys. Since the welds which join these are long (up to 25 $\mathrm{m}$ ) and straight, FSW is an ideal process, and the very low distortion is cited as a major benefit [46].

Apart from weld cracking, other problems with $7 \mathrm{xxx}$ and $2 \mathrm{xxx}$ alloys are corrosion and stress corrosion cracking (SCC). General corrosion resistance of aluminum alloys is usually an inverse function of the amount of copper used in the alloy. Thus, the 2xxx-series alloys are the least corrosion-resistant alloys, since copper is their primary alloying element, and all have appreciable (approximately $4 \mathrm{wt} . \%$ ) levels of copper [47]. For the same reason, resistance to general corrosion of the copper-free wrought $7 \mathrm{xxx}$ alloys is good, approaching that of the wrought $3 \mathrm{xxx}, 5 \mathrm{xxx}$ and $6 \mathrm{xxx}$ alloys but the copper-containing alloys of the $7 \mathrm{xxx}$ series, such as $7049,7050,7075$ and 7178 have 
lower resistance to general corrosion than those of the same series that do not contain copper. Generally, all $7 \mathrm{xxx}$ alloys are more resistant to general corrosion than $2 \mathrm{xxx}$ alloys, but less resistant than wrought alloys of other groups. It must be borne in mind despite the fact that the copper in both wrought and cast alloys of the aluminum alloys reduces resistance to general corrosion, it is beneficial from the standpoint of resistance to SCC [48]. However, both general corrosion and stress corrosion cracking depends on different parameters like pressure, temperature, UV radiation, humidity, chemical compositions, heat treatment, etc. Thus, it is not easy to predict how the corrosion and SCC will affect the performance in railcar application without having an extensive experimental study. This requires a deep research on metallurgical aspects of those alloys considering the operational factors and would be out of the scope of this thesis. But as both $2 \mathrm{xxx}$ and $7 \mathrm{xxx}$ series have been widely used in aerospace, military and some unique naval applications, which have much more erosive environment compared to railcar operational environments, it can be assured that with proper coating techniques these alloys can also be used in the rail sector.

\subsubsection{Aluminum-Lithium Alloys}

Due to the challenges with $2 \mathrm{xxx}$ and $7 \mathrm{xxx}$ series, it seems that aluminum-lithium alloy 8090 can be a better choice. The main advantages with this alloy are its low density due to the addition of lithium and improved corrosion resistance compared to $2 \mathrm{xxx}$ and 7xxx series. Alloy 8090 belongs to the family of second generation aluminum-lithium alloys. This specific alloy is representative of desired properties of aluminum-lithium alloys in general. In fact, trade-off studies showed that a reduction in density is the most advantageous way for weight reduction, and lithium, being the lightest metal, would have 
the greatest influence on reducing the density of aluminum [49]. In recent years, lightweight design requirements in the aerospace industry have made the aluminumlithium alloy systems an attractive choice due to their lower density, increased elastic modulus, increased fatigue crack growth resistance and increased corrosion resistance when compared to $7 \mathrm{xxx}$ and $2 \mathrm{xxx}$ series [50].

The first generation of Al-Li alloys was developed and commercially used in late 1950s by application of alloy 2020 in the RA-5C Vigilante Mach 2 aircraft wings and stabilizers. However, because of the low ductility (brittleness) of the first generation, they failed to meet the structural design requirements, resulting in no later commercial use $[51]$.

During the 1970s, the increase in fuel costs and landing weight fees resulted in a technical focus on weight reduction. This led to development of second generation Al-Li alloys [49]. The second-generation alloys certainly had improved mechanical properties when compared to the first-generation alloys. However, the properties still could not meet most aircraft specifications in thermal stability, anisotropy, ductility and weldability $[51]$.

Thermal Stability: Thermal stability is the ability of a material to maintain its mechanical properties when aged. All aluminum alloys have some thermal instability, but aluminum-lithium alloys tend to be more prone to it, their mechanical properties start to change at relatively low temperatures $\left(70-100^{\circ} \mathrm{C}\right)$. In aerospace applications, aerodynamic heating (heating due to friction with the air) and the heat produced by engine can also intensify the case [51]. This can lead to loss of toughness and ductility 
[49]. However, the latter factors might not be dominant in freight car application, as the speeds are much lower, and the engine is not even close to the wagons. But the effect of temperature increase during warm sunny days in summer on thermal stability should be considered.

Anisotropy: Anisotropy is the variance of mechanical properties of materials in different directions. For example, in cold or hot rolled materials, the strength properties in rolling direction are different from the lateral direction. Since highly anisotropic materials are harder to predict, most industries have little interest in them. Second generation Al-Li alloys tend to have anisotropic behavior, limiting their application in the industry [51].
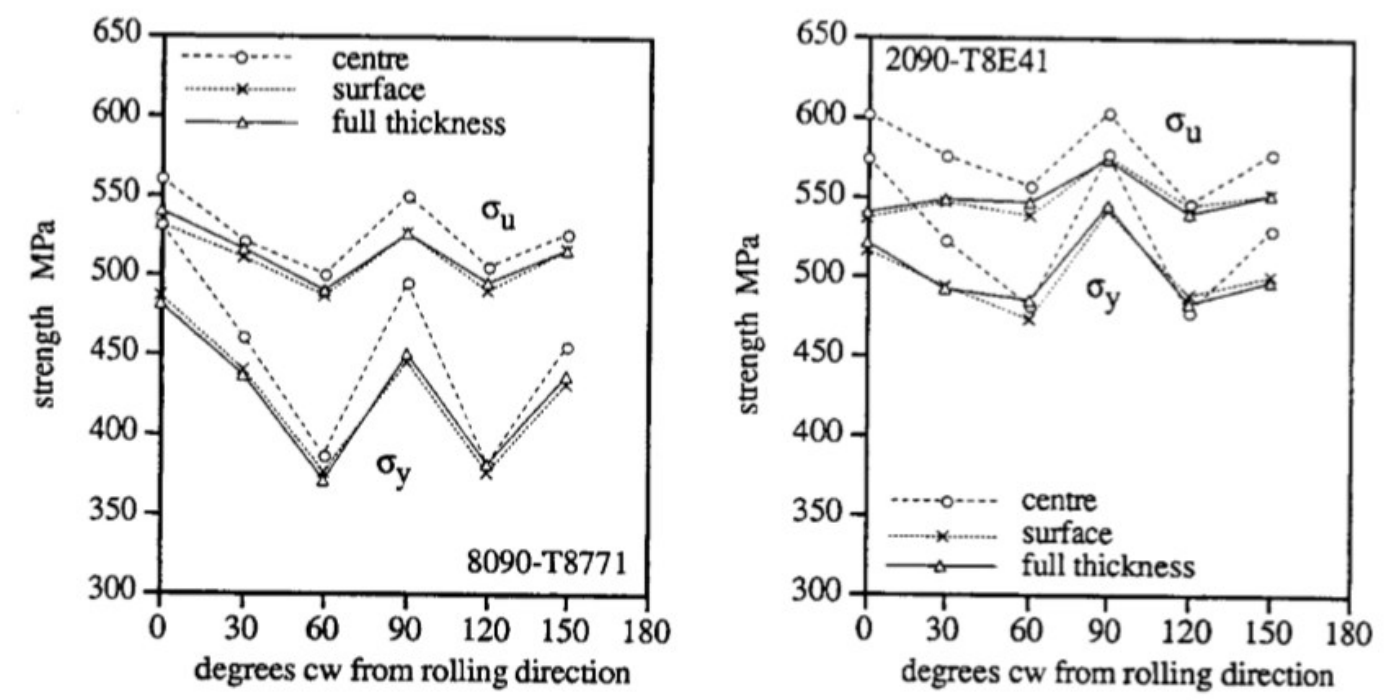

Figure 49: Ultimate tensile( $\left.\sigma_{u}\right)$ and yield strength $\left(\sigma_{y}\right)$ of 8090 and $2090 \mathrm{Al}-\mathrm{Li}$ alloys as a function of orientation [51] 
Weldability: Many of the high temperature welding methods such as gas arc welding plasma arc welding, electron beam, etc. result in some degree of strength loss in the welded joint. There are three reasons for this weakening. First, the heat results in the creation of pores in the heat-affected zone. Second, aluminum-lithium welded joints have a high susceptibility to hot cracking, and finally, heat treatment of the joint and heataffected zone is altered by the high temperatures [51]. These problems result from high temperature welding methods and can be attacked by using FSW as already discussed. Not only is it possible to weld these types of alloys using FSW, but also optimization of its processing parameters like weld speed and rotational speed have been well studied [52].

Since anisotropy can be used as an advantage for further weight saving in certain loading directions, it is not necessarily regarded as a drawback in structural design. Nevertheless, because of the other issues associated with second generation alloys, the third generation Al-Li alloys have started being developed in 1990s. Their main difference with second generation is the amount of lithium. In fact, second generation AlLi alloys have more than $2 \mathrm{wt} . \%$ lithium but this has reduced to less than $2 \mathrm{wt} . \%$ in the third-generation. By reducing the lithium content, many properties such as anisotropy, fracture toughness, weldability, crack deviation and thermal stability were improved [49, 51]. The mechanical properties of two sample alloys of third generation Al-Li system, 2099 and 2055, are listed Table 10. 
Table 10: Mechanical properties of Al-Li alloy 2099 and 2055 [53,54]

\begin{tabular}{|c|c|c|}
\hline Alloy class & $\begin{array}{l}2099 \\
\text { (T83) }\end{array}$ & $\begin{array}{r}2055 \\
\text { (T84) }\end{array}$ \\
\hline Density $\left(\mathrm{Kg} / \mathrm{m}^{3}\right)$ & 2630 & 2710 \\
\hline Yield Strength (MPa) & $\begin{array}{l}552^{(\mathrm{L})} \\
485^{(\mathrm{LT})}\end{array}$ & $\begin{array}{l}552^{(\mathrm{L})} \\
538^{(\mathrm{LT})}\end{array}$ \\
\hline Tensile Strength (MPa) & $\begin{array}{l}560^{(\mathrm{L})} \\
525^{(\mathrm{LT})}\end{array}$ & $\begin{array}{l}586^{(\mathrm{L})} \\
579^{(\mathrm{LT})} \\
\end{array}$ \\
\hline Elongation (\%) & $9^{\text {(L) }}$ & $\begin{array}{l}7^{(\mathrm{L})} \\
5^{(\mathrm{LT})}\end{array}$ \\
\hline Young's Modulus (GPa) & 78 & 76.5 \\
\hline Shear Modulus (GPa)* & 29.3 & 28.75 \\
\hline Poisson's Ratio & 0.33 & 0.33 \\
\hline
\end{tabular}

Alloy 2099 and 2055 were introduced in 2003 and 2011, respectively [49]. They are relatively new compared to conventional $2 \mathrm{xxx}, 6 \mathrm{xxx}$ and $7 \mathrm{xxx}$ alloys, and are finding their application in aerospace industry. There are many research works going on improving Al-Li alloys. Alloys 2099 and 2055 both have the desired mechanical properties and can be introduced as potential alternates for conventional aluminum alloys and steel in rail industry. The main drawback with these alloys is their higher material cost compared to conventional aluminum alloys. However, considering the 30 -year life time of hopper cars, this higher material cost can be compensated by potential fuel savings. This is like the case of conventional aluminum alloys and steel, where the higher price of aluminum (compared to steel) is compensated in 2 years due to the fuel savings 
resulted from weight reduction [41]. The use of Al-Li alloys can be even more financially justifiable, if Governmental regulations further restrict allowable $\mathrm{CO}_{2}$ emissions and mandate lower fuel consumption for rail transport.

Alloy 2055 has less anisotropy in its properties but the low elongation, especially in long transverse direction, is the main problem with this alloy. On the other hand, alloy 2099 has higher anisotropy in its properties but its higher elongation and lower density makes it an interesting candidate. In fact, alloy 2099 has higher specific stiffness compared to 2055. Although the strength (yield and tensile) of alloy 2099 is lower than 2055 , it is high enough to satisfy the design requirements. Finally, alloy 2099 is chosen to be the base material for further structural design.

There are some points regarding the properties of these alloys must be mentioned. First, anisotropic behavior is inevitable in most cold rolled sheet metal due to change in the orientation of the grain boundaries during rolling process. Of course, this is more evident in Al-Li alloys but to prevent any possible failures, the basis for further design would be the lowest values, i.e. values in long transverse direction. Second, all mentioned properties are subjected to change with the change in plate thickness and/or heat-treatment. Thus, in case better properties are required for design, thicker plates could be an option but here, the properties were derived for $7 \mathrm{~mm}$ thickness, same thickness as was used for steel hopper cars. Third, values listed in Table 10 were obtained from the provider's technical data sheet. In fact, producers usually provide the mean values obtained from testing different specimens but the same mechanical properties for the same materials might have lower values in hand books like "Metallic Materials Properties 
Development and Standardization (MMPDS)", MIL-HDBK-5 [55], as they use certain confidence limit for their statistical data obtained from different test results, see Figure 50. However, in this thesis the mean values (data provided by manufacturers), listed in Table 10, will be used.

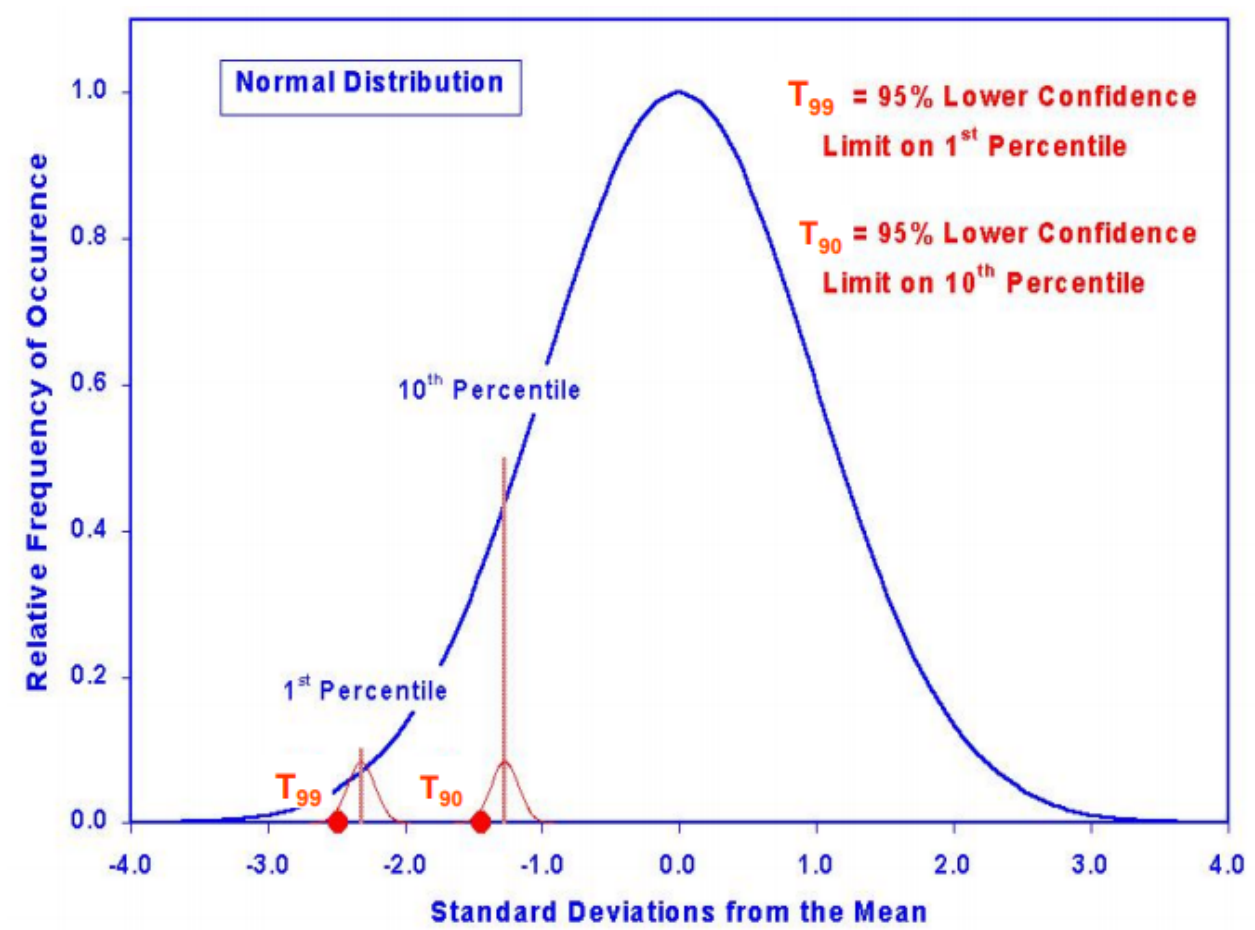

Figure 50: Confidence limit in MMPDS statistical data [56]

\subsection{Material Selection Summary}

With growing evidence of climate change and global warming, it will be increasingly difficult for the nations of the world to ignore these issues. Almost certainly, national transportation systems will strive to become as energy efficient and environmentally acceptable as possible [41]. In North America, the development of rail industry has lagged behind the rapid growth of the automotive and aerospace industry. 
This is because of a lack of both federal and state funding for major projects. Further, almost all the railroad tracks in the U.S. are privately owned by freight companies that are running slower, heavier trains, and these companies do not want to mix advanced, high-speed passenger trains with the slower moving freight trains. This situation is in contrast to that in many other European and Asian countries such as Germany, France and Japan, where the rail industry is driven by significant Government investments [41].

Therefore, in this chapter, a structured procedure is described for a lightweight material selection. It was proposed that magnesium alloys can be potential alternatives in future applications but currently, they are not applicable because of the problem with their flammability. It seemed that wrought aluminum alloys can be good candidates for heavy steel hopper cars. There are some reasons for using aluminum alloys over steel. First, the lighter aluminum railcar bodies, about two-thirds the weight of the comparable steel body, enable a greater payload. The higher payload capacity repays the higher initial cost of aluminum in less than two years [41]. In addition, the U.S. Department of Energy has reported that the conversion of steel cars to aluminum has significantly reduced the total $\mathrm{CO}_{2}$ emissions produced by the transportation sector [41]. Moreover, aluminum side walls offer improved aerodynamics, lower wind resistance and eliminate the need for paint as compared to steel walls, which will result in lower operating costs [41]. Finally, aluminum freight cars retain a high salvage value when recycled at the end of their life cycle. For instance, there was case which several hundred-ton hopper cars initially built around 1967 were sold for almost $90 \%$ of the original manufactured cost [41]. 
Despite the advantages that aluminum alloys can bring, they may have their own problems. These characteristics for different aluminum alloys are summarized in Table 11. Alloys 5xxx and 6xxx have been already used in high speed trains and cargo cars but their strength is not enough for this specific application. In addition, the aim was to select a high strength material to achieve more weight reduction and load capacity thorough appropriate structural design. This led the research to focus on $2 \mathrm{xxx}$ and $7 \mathrm{xxx}$ series. In fact, 7xxx series has also been limitedly used in high speed application. However, the problem with their weldability, corrosion and SCC make the situation complicated. Of course, there are coating techniques that can improve corrosion resistance and SCC, but the goal was to select the best material with the least possible challenges. Consequently, 8xxx series, specifically Al 8090, presented interesting characteristic include better corrosion resistance, higher SCC, better weldability and low density. This alloy attracted the attention toward a group of aluminum alloys called aluminum-lithium alloys. The best version of Al-Li alloys is the third generation with improved thermal stability, anisotropy, weldability, corrosion resistance and SCC. Finally, among third generation Al-Li alloys, alloy 2099 was selected as the material for this thesis, and further research will be conducted based on properties of this material. 
Table 11: Comparison of Aluminum Alloys

\begin{tabular}{|c|c|c|c|}
\hline Alloy & $\begin{array}{l}\text { Main Alloying } \\
\text { Element }\end{array}$ & Advantage & Disadvantage \\
\hline $1 \times x x$ & Pure & $\begin{array}{c}\text { Good Formability, } \\
\text { Weldability and Corrosion } \\
\text { Resistance }\end{array}$ & Low Strength \\
\hline $2 \times x x$ & Copper $(\mathrm{Cu})$ & $\begin{array}{l}\text { Good Machinability, High } \\
\text { strength }\end{array}$ & $\begin{array}{l}\text { Poor Weldability and } \\
\text { Corrosion Resistance, } \\
\text { Susceptible to Stress } \\
\text { Corrosion Cracking }\end{array}$ \\
\hline $3 \times x x$ & Manganese (Mn) & $\begin{array}{c}\text { Good Formability, } \\
\text { Weldability and Corrosion } \\
\text { Resistance }\end{array}$ & Moderate Strength \\
\hline $4 \times \mathbf{x x x}$ & Silicon $(\mathrm{Si})$ & $\begin{array}{c}\text { Good Formability, } \\
\text { Weldability and Corrosion } \\
\text { Resistance }\end{array}$ & $\begin{array}{l}\text { No Structural Application } \\
\text { (Fluidity) }\end{array}$ \\
\hline $5 \times x x$ & Magnesium (Mg) & $\begin{array}{c}\text { Good Formability, } \\
\text { Weldability and Corrosion } \\
\text { Resistance }\end{array}$ & Moderate Strength \\
\hline $6 \times x x$ & Magnesium (Mg) & $\begin{array}{c}\text { Good Formability, } \\
\text { Weldability and Corrosion } \\
\text { Resistance }\end{array}$ & Moderate Strength \\
\hline $7 \times x x$ & Zinc $(\mathrm{Zn})$ & $\begin{array}{l}\text { Good Machinability, High } \\
\text { strength }\end{array}$ & $\begin{array}{l}\text { Poor Weldability and } \\
\text { Corrosion Resistance, } \\
\text { Susceptible to Stress } \\
\text { Corrosion Cracking }\end{array}$ \\
\hline $8 \times x x$ & $\begin{array}{c}\text { Different } \\
\text { Materials but } \\
\text { Mostly Lithium } \\
\text { (Li) } \\
\end{array}$ & $\begin{array}{c}\text { Good Formability, Low } \\
\text { Density, High Strength, Good } \\
\text { Corrosion Resistance }\end{array}$ & $\begin{array}{l}\text { Poor Thermal Stability, } \\
\text { Anisotropic Properties }\end{array}$ \\
\hline
\end{tabular}

Due to the increasing application of composite materials in the industry, the aluminum companies are concerned about developing low-density alloys and improving aluminum metallurgy to keep the aluminum alloys competitive [49]. Although carbonfibre and boron-fibre nonmetallic composites can offer a significant weight savings over 
all other structural materials, improvements in the properties of aluminum alloys seemed desirable because of their relatively low acquisition cost and the industry's extensive design and manufacturing experience with these materials [49]. Finally, a general comparison between conventional aluminum alloys previously used in aerospace or rail industry, third generation Al-Li alloys, Carbon Fiber Reinforce Polymer (CFRP) and GLARE (Glass reinforced aluminum) is available in Table 12. 
Table 12: Comparison of Conventional Aluminum Alloys, Third-Generation Al-Li Alloys, CFRPs and GLARE [49]

\begin{tabular}{|c|c|c|c|}
\hline $\begin{array}{c}\text { Conventional Al } \\
\text { Alloys }\end{array}$ & I-Li & FRPs & ARE \\
\hline $\begin{array}{l}\text { ADVATNGES: } \\
\text { - Moderate } \\
\text { material, labor, and } \\
\text { manufacturing } \\
\text { costs } \\
\text { - Moderate specific } \\
\text { stiffness } \\
\text { - Isotropic } \\
\text { mechanical } \\
\text { properties } \\
\text { - Good property } \\
\text { control by } \\
\text { thermomechanical } \\
\text { processing } \\
\text { - Generally } \\
\text { recyclable } \\
\text { DISADVATNGES: } \\
\text { - Poor corrosion } \\
\text { resistance } \\
\text { - Stress corrosion } \\
\text { susceptibilities } \\
\text { - Most alloys } \\
\text { difficult or } \\
\text { unsuitable to weld } \\
\text { except with non- } \\
\text { fusion techniques } \\
\text { (like FSW) }\end{array}$ & $\begin{array}{l}\text { ADVATNGES: } \\
\text { - Moderate labor and } \\
\text { manufacturing costs } \\
\text { - 8-15\% higher specific } \\
\text { stiffness than conventional } \\
\text { aluminum alloys and } \\
\text { - Weldable: weight savings } \\
\text { and reduced part count } \\
\text { - Isotropic and improved } \\
\text { mechanical properties } \\
\text { - Good property control by } \\
\text { thermomechanical } \\
\text { processing } \\
\text { - Good/excellent corrosion } \\
\text { and stress corrosion } \\
\text { resistance } \\
\text { - Recyclable } \\
\text { DISADVATNGES: } \\
\text { - Higher material costs } \\
\text { compared to conventional } \\
\text { aluminum alloys } \\
\text { - Property dependences on } \\
\text { multistage } \\
\text { thermomechanical } \\
\text { treatments } \\
\text { - Separate recycling }\end{array}$ & $\begin{array}{l}\text { ADVATNGES: } \\
\text { - Higher and much higher specific } \\
\text { stiffness, depending on percentages } \\
\text { of aligned fibers } \\
\text { - } 10-20 \% \text { weight savings in actual } \\
\text { components } \\
\text { - High fatigue and corrosion } \\
\text { resistance: reduced maintenance } \\
\text { costs } \\
\text { DISADVATNGES: } \\
\text { - High material, labor and } \\
\text { manufacturing costs } \\
\text { - Possible delamination and other } \\
\text { flaws during fabrication } \\
\text { - Intrinsically anisotropic: complex } \\
\text { components difficult to analyze, } \\
\text { sometimes giving poor failure } \\
\text { predictions } \\
\text { - Higher notch sensitivity (e.g., } \\
\text { fastener holes) } \\
\text { - High susceptibility to damage } \\
\text { from impacts } \\
\text { - Damage growth difficult to } \\
\text { control and predict } \\
\text { - Difficult validation of repairs } \\
\text { - Flammable and non-recyclable }\end{array}$ & $\begin{array}{l}\text { - Fabricability } \\
\text { generally similar to } \\
\text { aluminum alloys } \\
\text { (but more } \\
\text { expensive, as stated } \\
\text { below) } \\
\text { - High strength: } \\
\text { weight savings in } \\
\text { tension-loaded } \\
\text { structures } \\
\text { - Excellent DT } \\
\text { (fatigue crack } \\
\text { growth, impact) } \\
\text { - Burn-through } \\
\text { resistance } \\
\text { DISADVATNGES: } \\
\text { - High material and } \\
\text { manufacturing costs } \\
\text { - Difficult to form } \\
\text { - Only sheet } \\
\text { products available } \\
\text { - Lower buckling } \\
\text { resistance: } \\
\text { unsuitable for } \\
\text { compression-loaded } \\
\text { structures }\end{array}$ \\
\hline
\end{tabular}




\section{Chapter 4. Structural Optimization}

\subsection{Introduction}

In the previous chapter, a light weight material with high strength (Alloy 2099) was introduced as an alternate to the conventional steel which has been traditionally used in hopper cars. However, there are some structural design techniques that can be applied to the railcar structure to obtain the most advantages from the new material and achieve even more weight reduction, while improving some of mechanical properties like specific stiffness. One of these techniques is to use sandwich panels instead of solid laminate or monolithic (monocoque) panels. Sandwich construction is a common principle in natural structures. Many of the bones in the skeletons of animals and humans are sandwich structures with foam-like core materials. Natural sandwich structures are subjected to complex load cases, while nature imposes a strict demand for lightweight primary structures like skeletons of birds [57].

Artificial sandwich structures were developed primarily to achieve lower weight and save fuel costs, and later, were adopted in response to restrict regulations in ecofriendliness, which forced industries to reduce emission and energy usage throughout the lifecycle of their products. It is also known that weight reduction is one of the most effective methods for energy saving but making a structure as light as possible without sacrificing strength is a fundamental challenge in design. In fact, the need for high performance, low-weight structures led to development of sandwich (hybrid) structures $[57,58]$. 
A sandwich panel typically is made from two face sheets that are attached to the core material by adhesive bonds. The functionality of a sandwich panel is like the classic I-beam, where two face sheets primarily resist the in-plane and lateral bending loads (similar to flanges of an I- beam), while the core material mainly resists the shear loads (similar to the web of an I-beam). Most often there are two faces, identical in material, fiber orientation and thickness but in special cases the faces may differ in thickness, materials, fiber orientation, or any combination of these three. The former sandwich is regarded as a mid-plane symmetric sandwich, and the latter a mid-plane asymmetric sandwich [58].

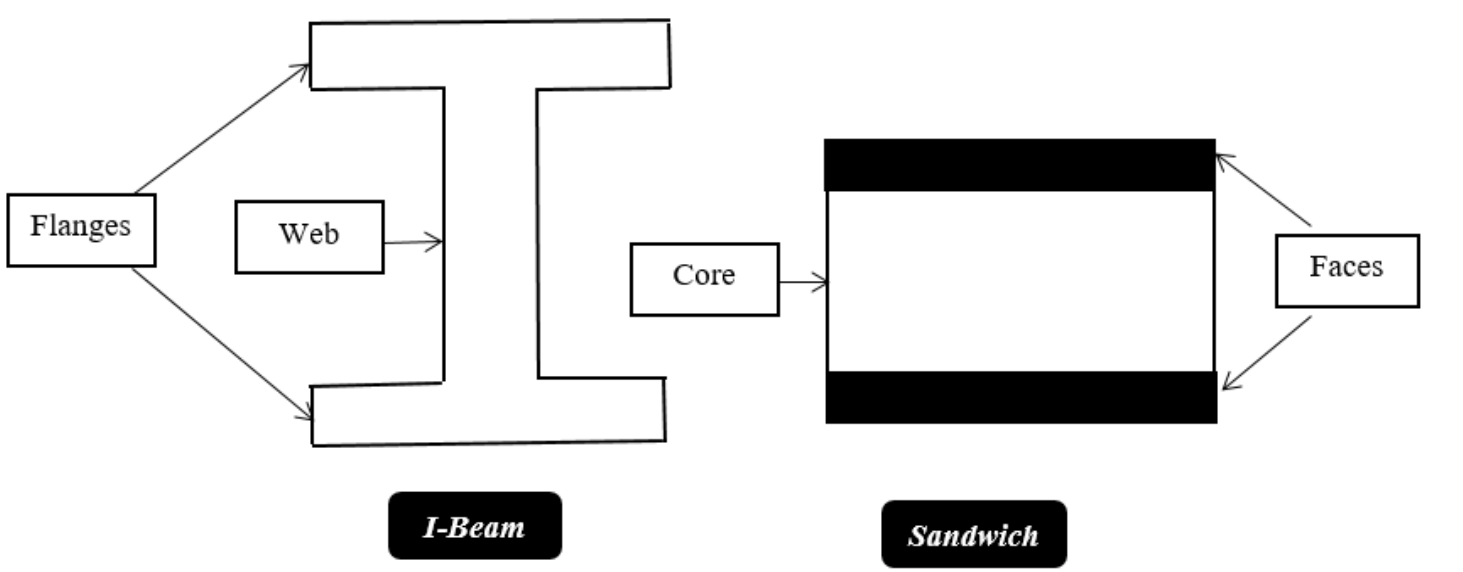

Figure 51: I-beam vs sandwich panel

The main point is that soft/light materials must be used for the core while relatively stronger materials are used for face sheets. This results in increasing the thickness of the panel, which often improves the structural attributes, while maintaining and/or reducing the weight [59]. This improvement in structural performance, specifically in bending stiffness, is mathematically shown below (see Figure 52) [59]: 


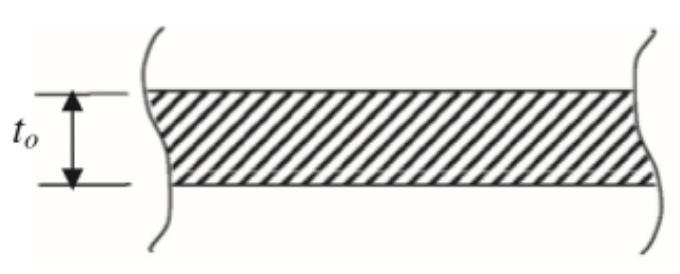

(a) Normal Panel

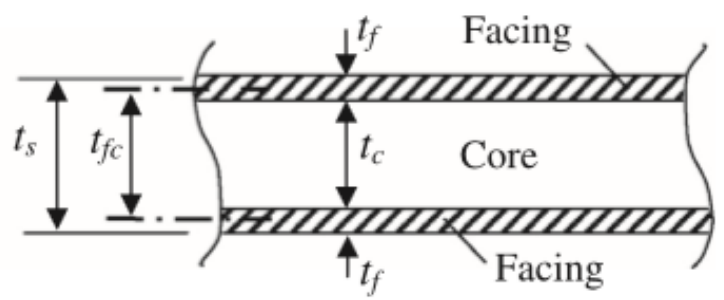

(b) Sandwich Panel

Figure 52: Schematic diagram cross-sectional view in (a) normal panel and (b) sandwich panel [59]

Where;

$t_{o}=$ Thickness of the normal panel

$t_{f}=$ Thickness of the facing material layers in the sandwich panel

$t_{c}=$ Thickness of the core material in the sandwich panel

$t_{s}=$ Overall thickness of the sandwich panel $\left(t_{s}=t_{c}+2 t_{f}\right)$

$t_{f c}=$ Distance between face sheet centroids in the sandwich panel $\left(t_{f c}=t_{f}+t_{c}=d\right)$

$E_{o}=$ Elastic modulus of the base material in normal panel

$E_{f}=$ Elastic modulus of the facing material layers in the sandwich panel

$E_{c}=$ Elastic modulus of the core material in the sandwich panel

$I_{o}=$ Moment of Inertia of the normal panel

$I_{f}=$ Moment of Inertia of the facing layer in the sandwich panel

$I_{c}=$ Moment of Inertia of the core in the sandwich panel

Assuming unit width and length-span, the bending stiffness of original normal panel is calculated as [59]:

$D_{o}=E_{o} I_{o}=\frac{1}{12} E_{o} t_{o}^{3}$

The bending stiffness of the sandwich panel is calculated as [59]: 
$D_{s}=E_{c} \cdot I_{c}+2 E_{f} \cdot I_{f}=\frac{1}{6} E_{f} \cdot t_{f}^{3}+\frac{1}{2} E_{f} \cdot t_{f} \cdot d^{2}+\frac{1}{12} E_{c} \cdot t_{c}^{3}$

Even if an identical material is assumed for the normal panel, face sheets and core, the elastic modulus of the sandwich core is not equal to its base material because of the core's soft nature and special topology, but that is not the case for face sheets $\left(E_{f}=\right.$ $\left.E_{o}=E\right)$. As a case study, consider the case where $t_{s} / t_{o}=2$ and $t_{o} / t_{f}=3$. If the

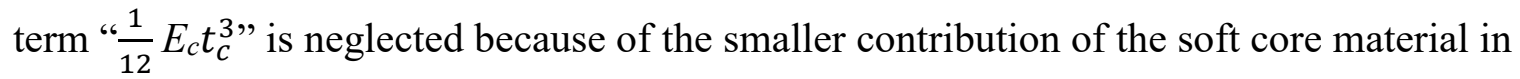
bending stiffness $\left(E_{c}<<E_{o}\right)$, then:

Table 13: Weight saving and structural performance potential of sandwich panel

\begin{tabular}{|c|c|c|c|}
\hline Property & Monolithic panel & Sandwich panel & Relative Value \\
\hline Bending stiffness & $2.25 E_{o} t_{f}^{3}$ & $12.66 E_{o} t_{f}^{3}$ & $D_{s} / D_{o}=5.66$ \\
\hline Weight & $3 \rho_{o}$ & $2 \rho_{o}+4 \rho_{c}$ & $W_{s} / W_{o}=0.66$ \\
\hline
\end{tabular}

Where;

$\rho_{o}=$ Density of the base material in single panel

$\rho_{f}=$ Density of the face sheets in sandwich panel $\left(\rho_{f}=\rho_{o}\right)$

$\rho_{c}=$ Density of the core material in sandwich panel $\left(\rho_{c}<<\rho_{o}\right)$

$W_{s}=$ Weight of the sandwich panel

$W_{o}=$ Weight of the single panel

As is shown in Table 13, the bending stiffness of a sandwich panel with the double thickness of a monolithic panel, is 5.66 times higher compared to a single panel, while $34 \%$ weight reduction is achieved. This example showed that how a sandwich construction can improve the structural behavior while reducing the weight. To sum up, it 
can be said that "for a given lateral load the sandwich construction results in a much lower lateral deflection, much higher overall buckling load, and much higher flexural natural vibration frequencies than does the monocoque construction of nearly the same weight" [58].

The above was just an example to better illustrate the capabilities of sandwich structures but the advantages of using them over normal panels has been pointed out in different research works. For instance, it is reported that the maximum bending stress in face sheets of a sandwich construction (face thickness to core depth ratio equal to $1 / 20$ ) is $1 / 30$ the maximum stress at the surfaces of the monocoque construction subjected to the same bending moment [58].

The abovementioned structural characteristics make the sandwich construction a very desirable technique for wide variety of applications. On the other hand, there are some drawbacks associated with this type of construction including difficult damage inspection of the core, moisture trap in unit cells, delamination and higher manufacturing cost are some examples of these drawbacks. The main point is that their advantages strongly outweight the disadvantages, and that is why many of industries like aerospace, automotive and navy are moving toward using this type of technique in their structures. Despite the weight savings that a sandwich construction brings the structure, it still must be optimized for specific applications in order achieve the most structural efficiency. This starts with an appropriate selection of the core topology. 


\subsection{Types of Cellular Core}

The very first step in developing a sandwich structure is to choose a suitable core configuration for the specific application. The core of a sandwich structure can be of almost any material or architecture [58], and hence, each research work categorizes cores in a different way. However, the main idea behind all these classifications is the same. A generic classification of typical manmade cellular cores is shown in Table 14.

Table 14: Classification of cellular cores

\begin{tabular}{|c|c|c|c|c|}
\hline \multicolumn{5}{|c|}{ Cellular Core } \\
\hline \multicolumn{2}{|c|}{ Stochastic } & \multicolumn{3}{|c|}{ Periodic } \\
\hline Open-Cell & Closed-Cell & Closed-Cell & Oper & ttice) \\
\hline & & Honeycomb & Truss & $\begin{array}{c}\text { Woven } \\
\text { (Textile) }\end{array}$ \\
\hline 2 & 2 & 3 & 3 & [60] \\
\hline
\end{tabular}

There are some points regarding the above classification that must be identified.

First, only most popular sub-categories were mentioned above. In fact, both periodic and stochastic types have other sub-categories than what was shown above but they are not

\footnotetext{
${ }^{2}$ Foard Panel, “Open Vs. Closed Cell Foams”, [Online]. Available: http://www.foardpanel.com/open-vsclosed-cell-foams/

${ }^{3}$ Wadley Research Group, University of Virginia, "Ultralight Cellular Materials", [Online]. Available: http:/www.virginia.edu/ms/research/wadley/celluar-materials.html
} 
widely used for structural applications. Second, the examples shown in the above table are just sample topologies, i.e. many different topologies exist for both honeycomb and truss core. For instance, for a honeycomb core different shapes like square, hexagonal and triangular have been developed, and in case of open cell lattice (truss), different geometries like tetrahedral, pyramidal and Kagome have been designed. Finally, it worth mentioning that using solid cores is also possible but not common, as the main objective of developing sandwich panels is to reduce weight, and this can be better achieved by using a cellular core instead of a solid. That is why a solid type core is usually ignored in classifications.

There are three main principles involved with a cellular core which affects the overall performance of a panel [61]:

(i) The properties of the solid material of which the core is made.

(ii) The topology (connectivity) and shape of the cell edges and faces.

(iii) The relative density of the core with respect to its base material $\left(\rho_{r}\right)$.

The base material was selected in the previous chapter, and it is decided to use an identical material, i.e. Al 2099, for both core and face sheets because as was stated before, the focus of this project is on metallic materials, and the selected material has a better weight saving potential compared to its alternatives like steel or conventional aluminums. In addition, with the increasing focus on environmental protection legislation, reducing waste becomes an important aspect for the transport industry. From that perspective, fully aluminum sandwich panels are considered as an attractive case even compared to non-metallic materials like polymer composite sandwich panels [62]. Therefore, the first part, selection of the base material, has been already discussed in 
Chapter 3. The discussion in this chapter focuses on the second and third part. In another words, the main focus in this chapter is to describe the selection of the best type of core, select the best topology within that specific core family, and finally, optimize the selected topology to achieve the minimum weight. As a result, the first step is to decide about the type of core.

\subsection{Comparison of Cellular Cores}

It has been stated in different research works that lattice cores may structurally outperform the foam cores in terms of strength and stiffness $[63,64]$. In fact, the structural applications of foam cores have been limited due to their low strength and stiffness, and their load supporting capabilities are greatly inferior to those of honeycombs of the same density [63]. Moreover, because of the random topology of foam materials it is difficult to tailor mechanical properties, like strength and stiffness, for different applications. Therefore, even though cell foams have shown promise for impact energy absorption, acoustic damping and thermal insulation, their weaknesses have generated significant interest towards lattice cores for load supporting applications [63]. The reason behind their low strength and stiffness, can be found in their nodal connectivity which leads to another form of core classification, i.e. "bending dominated" and "stretch dominated".

Generally, bending dominated structures known to have low connectivity of the joints (the number of struts that meet at joints), and this causes the cell edges to bend when the structure is loaded. On the other hand, stretch dominated structures have better nodal connectivity which enables their cell edges to stretch when loading, this results in 
higher stiffness and strength [63]. To sum up, it is said that stretch dominated structures have high structural efficiency, whereas bending dominated structures have low efficiency [61]. This thesis is not intended to deeply study the bending dominated or stretch dominated cores and their equations. In fact, the only reason that the matter was discussed is to use it as a guideline for selecting the best core for the freight car application. The governing equations for each type of lattice core are listed in Table 15.

Table 15: Governing equations for bending dominated and stretch dominated lattices [61]

\begin{tabular}{|c|c|c|}
\hline Bending dominant & Stiffness & Strength \\
\hline (e.g. Foam, woven) & $\left(\frac{E_{c}}{E_{o}}\right) \alpha\left(\frac{\rho_{c}}{\rho_{o}}\right)^{2}$ & $\left(\frac{\sigma_{y c}}{\sigma_{y 0}}\right) \alpha\left(\frac{\rho_{c}}{\rho_{o}}\right)^{3 / 2}$ \\
\hline $\begin{array}{c}\text { Stretch dominant } \\
\text { (e.g. Honeycomb, Pyramidal truss) }\end{array}$ & $\left(\frac{E_{c}}{E_{o}}\right) \alpha\left(\frac{\rho_{c}}{\rho_{o}}\right)^{1}$ & $\left(\frac{\sigma_{y c}}{\sigma_{y o}}\right) \alpha\left(\frac{\rho_{c}}{\rho_{o}}\right)^{1}$ \\
\hline
\end{tabular}

Where;

$\sigma_{y o}=$ Yield strength of the base material that the core is mad from

$\sigma_{y c}=$ Yield strength of the core

The above governing equations are summarized in Figures 53 and 54, in which the relative modulus $\left(\frac{E_{c}}{E_{0}}\right)$ and strength $\left(\frac{\sigma_{y c}}{\sigma_{y, 0}}\right)$ are plotted on logarithmic scales against relative density $\left(\frac{\rho_{c}}{\rho_{0}}\right)$. These plots can better illustrate the performance of each cellular core in comparison with other candidates. 


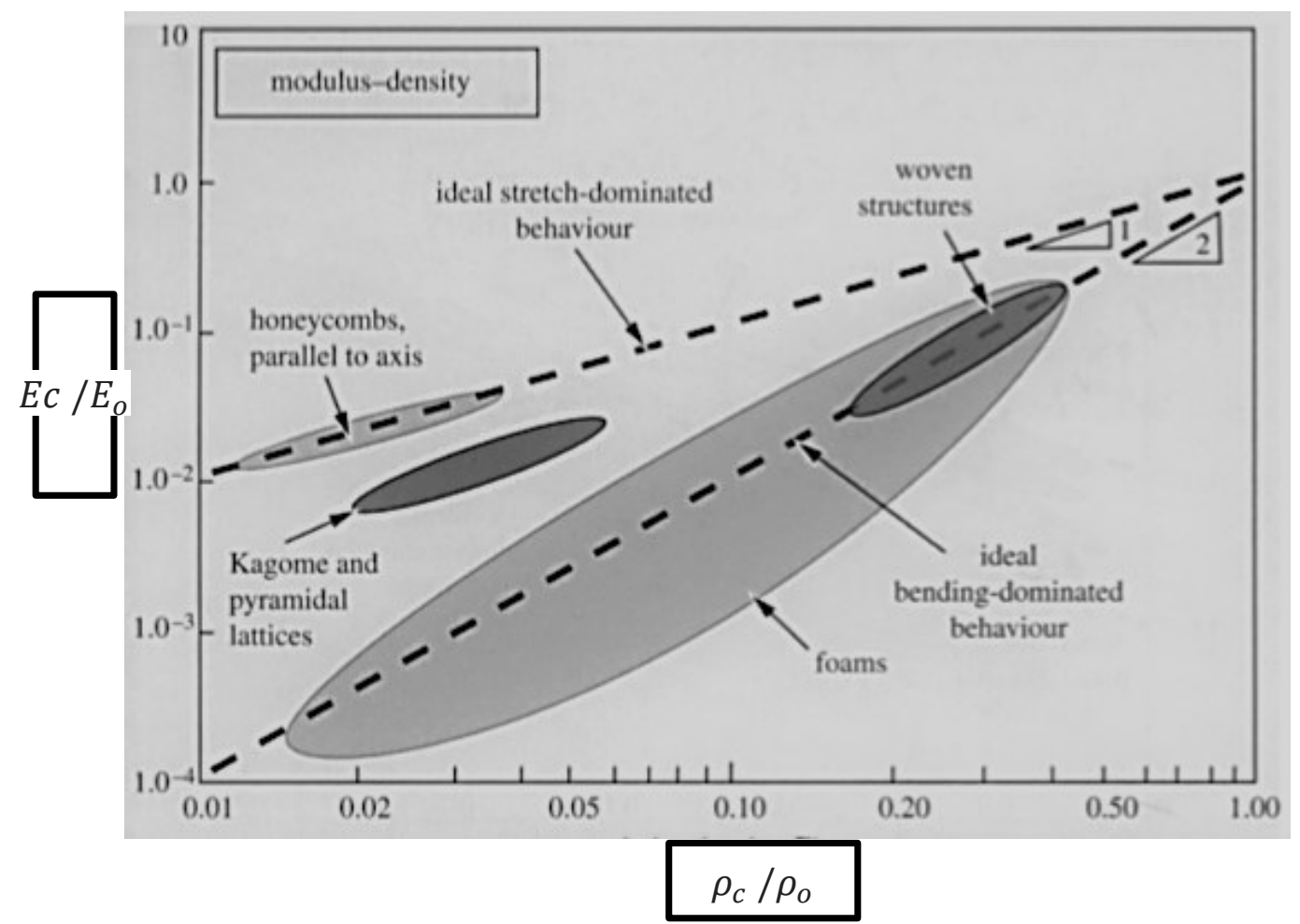

Figure 53: Relative modulus vs relative density on logarithmic scales for cellular structures [61]

As can be seen in Figure 53, the two broken lines represents the ideal stretch and bending dominated lattices in the graph. Stretch dominated mesostructures have moduli that scale as $\left(\frac{\rho_{c}}{\rho_{0}}\right)$ (slope 1), while bending dominated cellular mesostructures have moduli that scale as $\left(\frac{\rho_{c}}{\rho_{o}}\right)^{2}$ (slope 2). Woven structures and foams fall below honeycomb and truss lattices when it comes to stiffness. In fact, most of the foams lies on the ideal bending dominated line, while some of them fall below. That is because of the heterogeneous nature of foam cores, it means that they are strong in some places and weak in others. The weak regions result in a deviation from the ideal line. Honeycombs behave as an ideal stretch-dominant structure, when they are loaded parallel to the hexagon element, i.e. out of plane loading. They are also extremely compliant in loading other directions. Finally, truss lattices, both Kagome and all types of pyramidal lattices, have stretch 
dominated properties. They lie close the ideal stretch dominated line but are not as efficient as honeycombs and fall below the line for honeycombs.

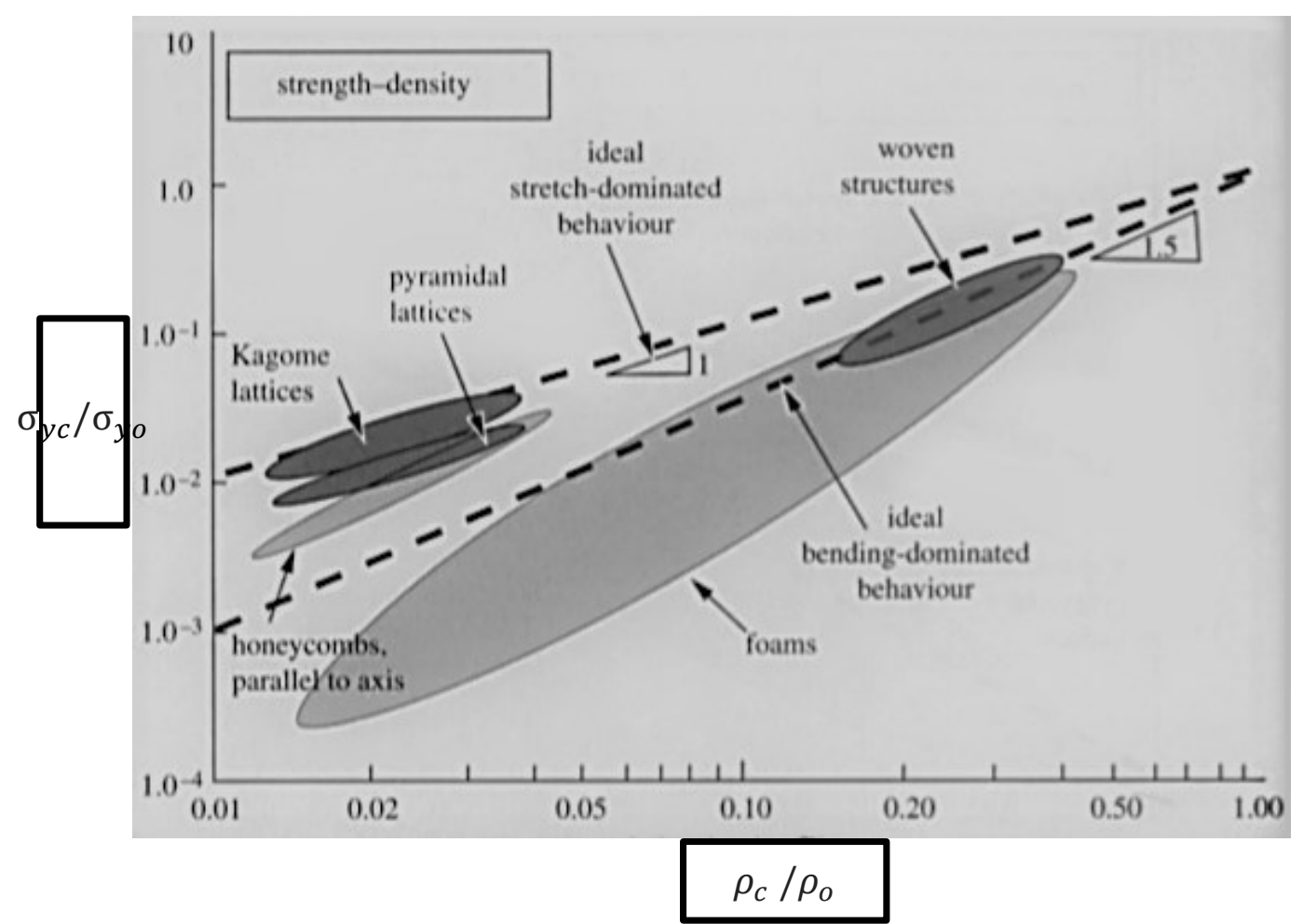

Figure 54: Relative strength vs relative density on logarithmic scales for cellular structures [61]

The strength plots have much in common with stiffness plot but there are some notable differences. As before, the ideals are show as dashed lines. Stretch-dominated mesostructures have strengths that scale as $\left(\frac{\rho_{c}}{\rho_{0}}\right)$ (slope 1), while bending-dominated scale as $\left(\frac{\rho_{c}}{\rho_{o}}\right)^{3 / 2}$ (slope 1.5). Woven structures have ideal bending dominated behavior, while the foams underperform. In fact, almost none of the foams could reach the ideal line as a result of their imperfections. Honeycombs also fall below the ideal stretch dominated line because their cell walls easily buckle in compression. Like stiffness, truss lattices have 
near ideal stretch dominated performance. More specifically, the Kagome lattices seem to have the most efficient performance when it comes to strength [61].

As is obvious from the above discussion, truss lattices and honeycombs would definitely outperform the foams and woven structures, but their own performances are comparable. When it comes to stiffness, the honeycombs are the ideal cores and the truss lattices fall below them. On the other hand, in designs where strength is the limiting parameter, the truss lattices are the most efficient ones and honeycombs underperform as compared to the ideal stretch dominated line. In fact, both stiffness and strength are desirable properties for the freight car application but as was discussed earlier, the most important thing in design of this type of rail cars is the strength. From this perspective, the truss lattices seemed to be better choices. In addition, truss lattices can improve other shortcomings of honeycombs such as their susceptibility to corrosion and delamination because of entrapped moisture [65]. Moreover, truss cores provide additional opportunities for multifunctional usages like acoustic damping and high intensity dynamic load protection [64]. Although the intense impact loads are not supposed to be tolerated by the railcar body, this function brings a potential for more overall weight saving in supporting structures like center sills, bogie, etc.

There have been some studies on developing honeycomb sandwich structures but this thesis is trying to tackle a relatively novel application. That is developing and optimizing a truss core sandwich panel for the cargo type railcars, specifically hopper cars. The calculations and analysis might be for a specific type of truss core with specific loading associated with hopper cars but the idea can be applied to any other type of 
freight car. In a more general sense, the technique is applicable for any thin walled structure with a given truss lattice topology that is subjected to lateral loading.

\subsection{Truss Lattices}

It was concluded that the truss core can be the best choice for a sandwich panel in freight car design. The next step is to select a generic topology for the truss core. Nowadays, with the aid of additive manufacturing (e.g. selective laser sintering) it is possible to manufacture truss lattices with almost any arbitrary topology. However, this may not be practical for industrial applications, as it would be too expensive to manufacture for example a whole $3 \mathrm{~m} \times 8 \mathrm{~m}$ floor panel with additive manufacturing. Therefore, only the most common truss topologies which can be manufactured with conventional methods will be considered as candidates, i.e. tetrahedral, pyramidal, octet and Kagome lattices. In fact, the manufacturing cost would be a determinative factor in selection process because it does not make sense to suggest an idea which is too challenging, expensive or financially unjustifiable. Especially, since the freight car industry is not very competitive, and the performance is not as critical as it is in the aerospace or automotive industries, the trend is toward methods that can satisfy the requirements, like the Government regulations for emission, with the least possible cost and complication.

Kagome lattices, shown in Figure 55, are superior compared to other common truss lattice topologies but they are more complex in fabrication. One option is to use injection molding to create a wax or polymer template of the truss core that can be used as a sacrificial pattern for investment casting. This requires the fabrication of a complex 
and often expensive die. Furthermore, if a design change in the sandwich panel has to be made, a new die has to be made with additional costs [66]. The other option is rapid prototyping which is not feasible as was discussed above. Consequently, despite their better performance compared to other truss lattices, Kagome lattices will be eliminated from the list of candidates [66]. Once again, it must be mentioned that there are many different possible topologies for truss lattices but the ones that are most frequently used in the literature are tetrahedral, pyramidal and octet, shown in Figures 56-58. The manufacturing process of these lattices will be discussed in more detail.

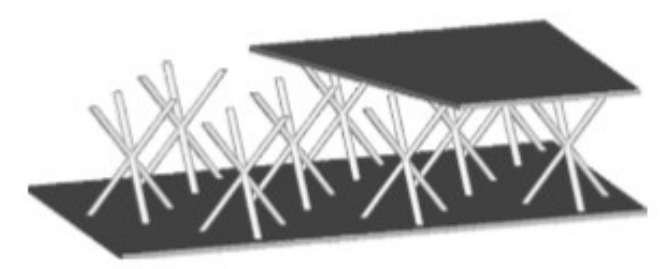

Figure 55: Kagome truss lattice [64]

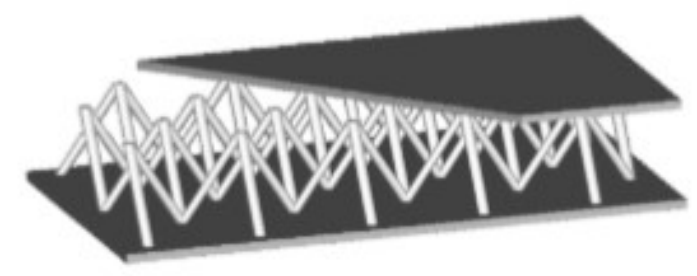

Figure 56: Tetrahedral truss lattice [64]

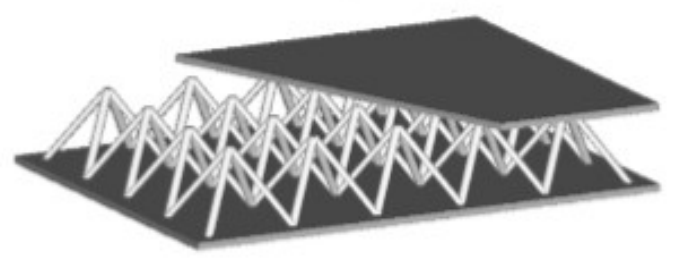

Figure 57: Pyramidal truss lattice [64] 


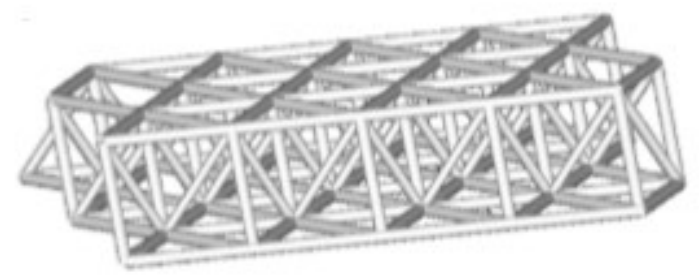

Figure 58: Octet truss lattice [64]

Apart from expensive methods like additive manufacturing or investment casting, there is another method which can be considered as conventional forming process. This fabrication process is very similar for both tetrahedral and pyramidal lattices. The schematic of the process is shown in Figures 59 and 60.

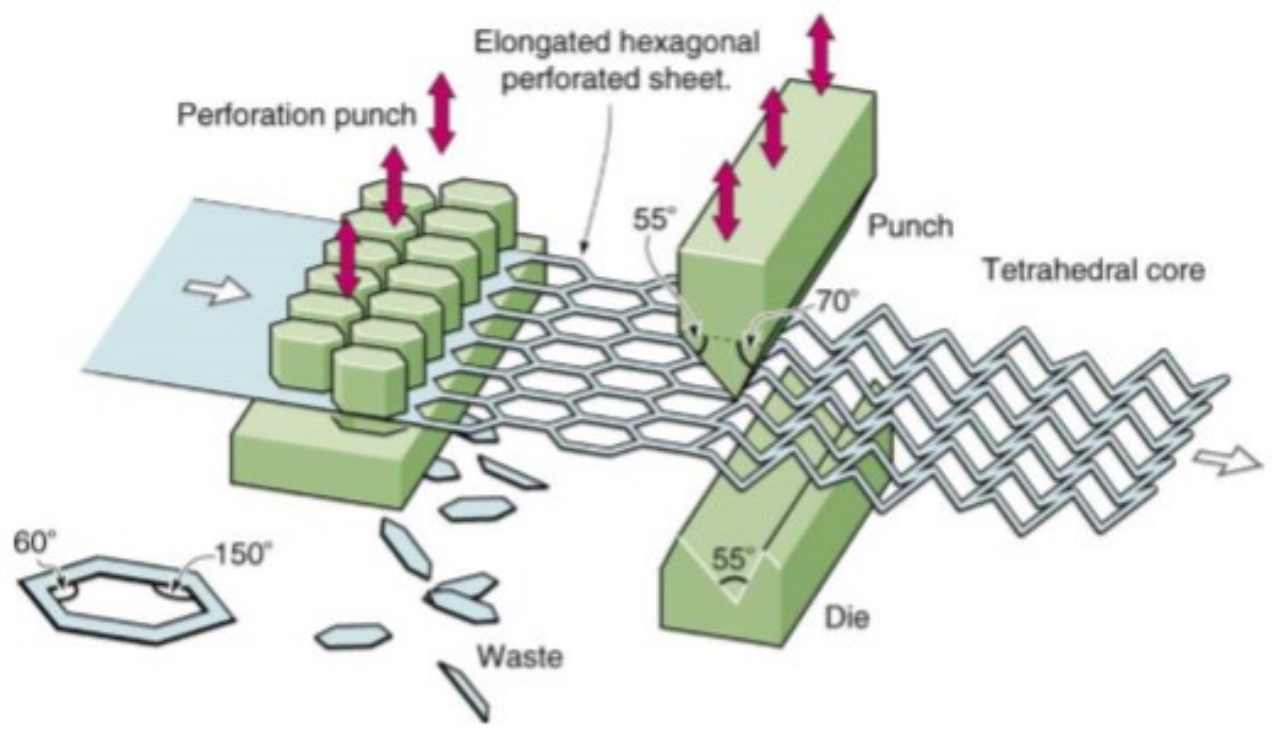

Figure 59: Schematic of the manufacturing process of the tetrahedral lattice truss cores [64] 


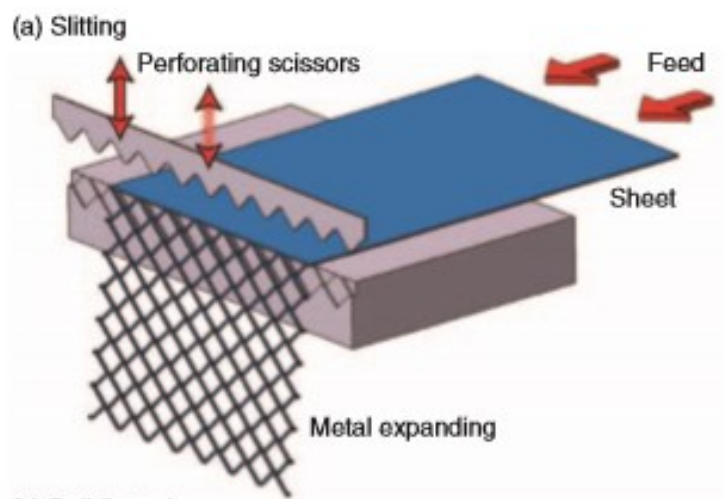

(b) Roll flattening

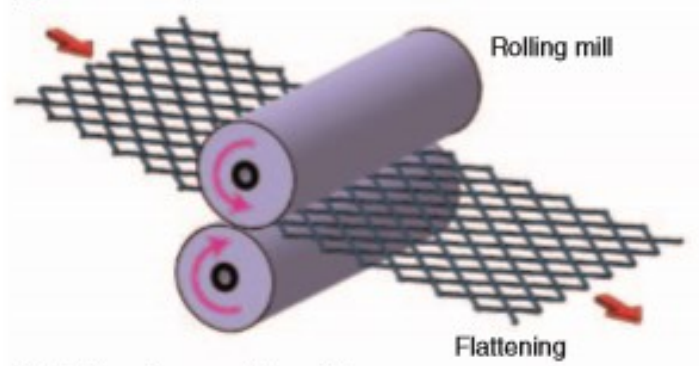

(c) Folding of expanded metal

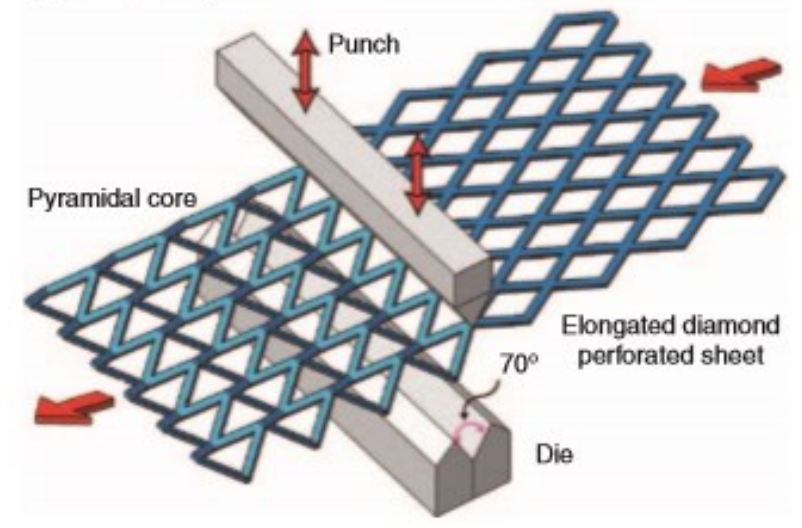

Figure 60: Schematic of the manufacturing process of the pyramidal lattice truss cores [66]

This manufacturing method consists of two main steps [64, 65]:

i. Sheet perforation: Perforating an extended metal sheet. For a tetrahedral core, the sheet must be hexagonally perforated while in case of pyramidal core, the perforation process should create diamond shapes.

ii. Node folding: Node row by node row folding using a paired punch and die tool. 
It has to be noted that a roll flattening process can be followed by sheet perforation, in order to achieve the desired truss size and cross-sectional shape [65]. Nevertheless, these features can be achieved by appropriately selecting the sheet metal thickness or spacing the perforating punches [64]. Depending on the type of material used for the core and face sheets, different bonding methods exist. Furnace brazing method known to be the common method for aluminum alloys, while for many stainless steels, super alloys and copper alloys a transient liquid phase (TLP) process has been successful [67].

The manufacturing process of octet lattices is slightly different from what was stated for tetrahedral or pyramidal ones. Its consists of four steps [68]:

i. Lattice truss fabrication: The truss row patterns are water jet cut from as-received sheets forming the geometries shown in Figure 61(a).

ii. Pyramidal truss assembly: Rows of trusses are collinearly aligned and a second collinear array, oriented at 90 to the first, was snap-fit attached to their tops forming a [0/90] arrangement of trusses enclosing a pyramidal void shape, Fig. 61(b). Control of the slot tolerance in the nodal regions enabled a mechanically stable snap-fitted plane of pyramidal trusses to be assembled in this way.

iii. Intermediate face sheet fabrication: The intermediate face sheet is water jet cut from as-received sheets forming the geometries shown in Figure 61(c). 
iv. Final octet truss Assembly: The truss layer planes were then snap-fitted into the crosses of the intermediate face sheet and the process repeated to form the octet-truss lattice, Fig. 61(d)

(a) Lattice truss fabrication

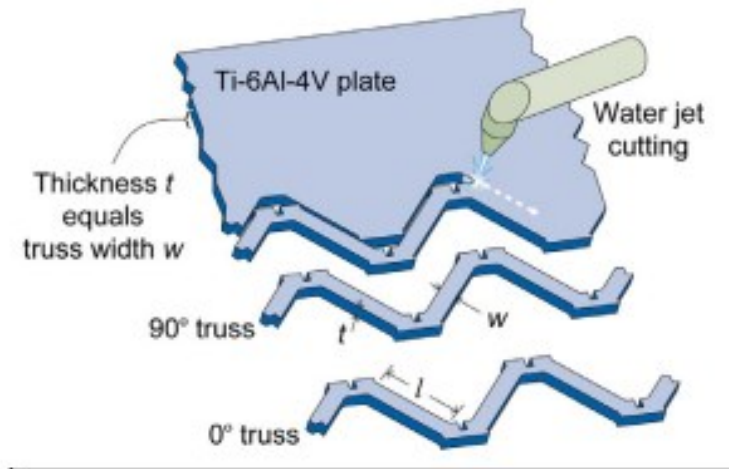

(c) Open face sheet fabrication

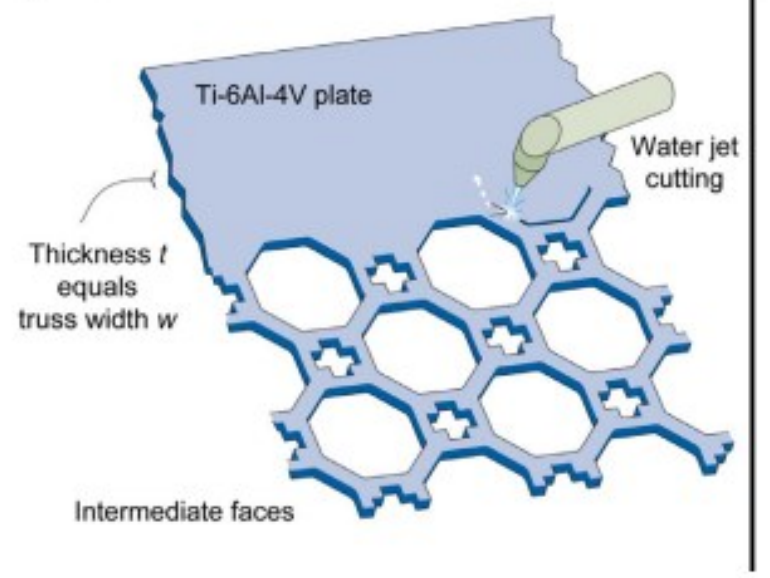

(b) Truss assembly

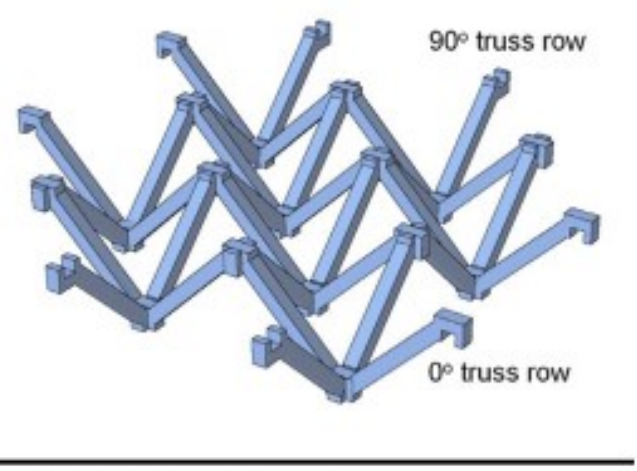

(d) Octet-truss lattice assembly

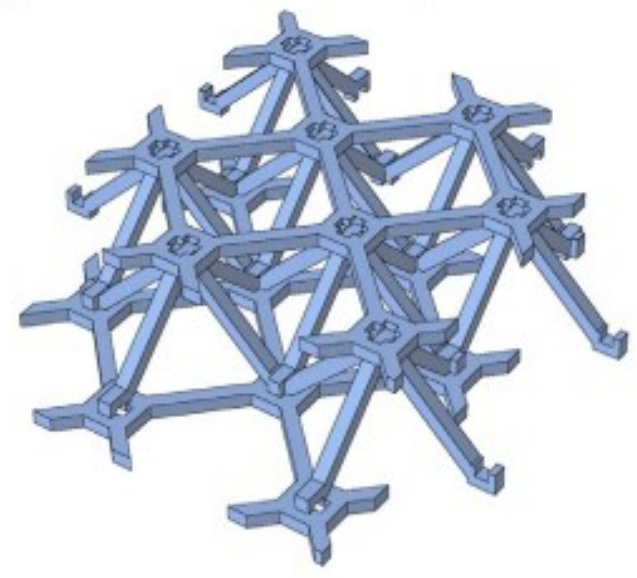

Figure 61: Schematic of the manufacturing process of the octet lattice truss cores [68]

In the above example, the water jet cutting was used because the base metal was Ti-64 which known to be a hard metal, and is not easy to perforate this metal with stamp/punching process or even other cutting method like plasma or laser cutting. In fact, the advantage of water jet cutting is that it can be used for a wide variety of materials. This is more useful in aerospace industry where materials like carbon fiber composites or 
titanium alloys might be used. However, in this study as the base metal is aluminumlithium alloy, other less expensive methods like plasma cutting, laser cutting or perforation punching can be substituted for fabrication of intermediate planes. Moreover, it is even possible to produce the final assembly of pyramidal lattices, Fig. 61(b), with the same conventional forming method. In fact, for aluminum alloy or steel plates, the mentioned process can be simplified as:

i. Pyramidal lattice fabrication: This includes sheet perforation and nodal folding in the same manner that was explained for pyramidal lattices.

ii. Intermediate sheet fabrication: Perforating an extended aluminum-lithium alloy sheet using perforation punch.

\section{iii. Final assembly}

\subsection{Case Study}

From the previous discussion, it is concluded that for materials commonly used in the rail industry, like aluminum or steel alloys, the manufacturing costs of octet lattices would not be much higher than other lattice core topologies. However, the regular octet truss lattice has an interesting feature which makes it a desirable candidate, i.e. fully isotropic behavior [69]. As a result, the regular octet truss core is selected for this thesis.

Since the design of a new sandwich structure is a daunting and demanding task and associated with many different features that must be considered, the focus of this study will be on the floor panel. In fact, this would be a preliminary structural design focused on developing a novel octet-core sandwich structure for the floor panel, but it can 
be used as a guideline for developing sandwich constructions for other parts of the railcar body or any other thin-walled structure subjected to lateral loading.

The aim is to design a sandwich panel with minimum weight. This includes defining the length of struts and their cross-sectional area in mesoscale design, and finding the thickness of the core and face sheets in macroscale design. Therefore, the process is a combination of sizing design and structural optimization. Of course, the structural optimization can be much broader than what will be done in this thesis but as was stated earlier, this is only a preliminary design focused on sizing and developing an application specific sandwich panel. The resulting structure can be further designed (or optimized) with more details but that would be out of the scope of this study. For that reason, the cross section of lattice struts is assumed to be circular, i.e. the cross-sectional shape is already fixed and there would be no shape optimization. Additional information on mapping of mechanical properties versus cross sectional shape can be found in other papers, like [70]. It has to be noted that many of these structurally desirable crosssectional shapes are not suitable from fabrication point of view because they can only be produced with expensive methods like rapid prototyping and investment casting. That is why the present research is concentrated on an easily manufactured shape (solid circular), despite the fact that hollow struts shown to have better weight saving potentials [63].

\subsection{Design Scale}

In design of sandwich structures with lattice cores, two different scales must be considered: macroscale design and mesoscale design. Macroscale design deals with the overall performance of the panel and its failure mechanism like failure of face sheets due 
to tensile stress or failure of the core because of shear stress. However, there is another aspect in structural design that has to be separately investigated and is called mesoscale design. It deals with the design of a lattice core unit cells. Unit cell (truss) is the building block of the lattice material, and it consists of finite number of members called struts. The failure mechanism of these struts might be different from the overall failure mechanism of the structure, and that is why they must be studied separately. Since these struts are much smaller than the whole sandwich structure, the mesoscale design also known as microscale design in the literature. Before starting the design, it is necessary to know what modes of failure might occur in sandwich structures. Then, these modes can be categorized as mesoscale or macroscale failure for further investigation.

\subsection{Failure Modes}

Various failure modes for a sandwich panel exist but not all those modes are of concern for a railcar floor panel. The detailed loading condition was stated in Chapter 2 but as a reminder, the floor panel is subjected to uniform lateral load. This load results in bending moment and shear forces in the structure. The bending moment leads to tensile and compressive stresses, while the shear load results in shear stress, shown in Figure 62. 

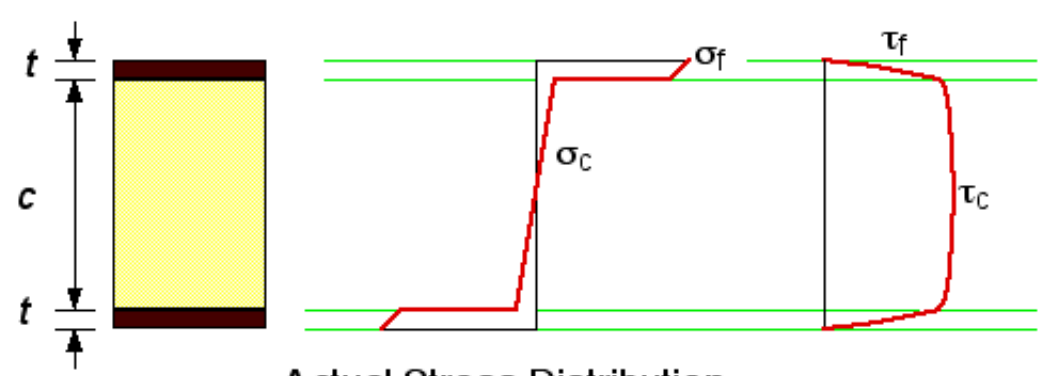

Actual Stress Distribution

Nonlinear - varies through thickness
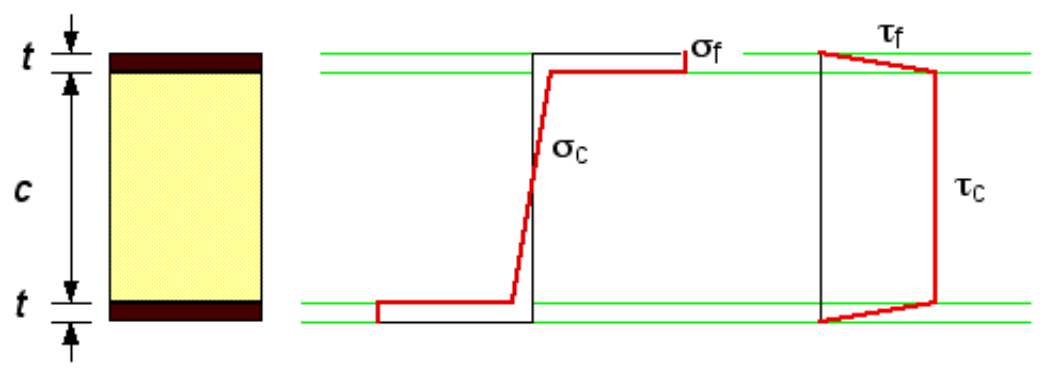

$\underset{\text { Linear or Constant }}{\text { Approximate Stress Distion }}$

Figure 62: Stress distribution in sandwich structure as a result of uniform lateral load [71]

Whether to use the approximate or actual stress distribution, which will be discussed later, the nature of the stresses in the panel would not change. These stresses will determine the failure modes associated with lateral loading. A list of all possible failure modes in a sandwich structure with their causes is shown in Figure 63 [72].

As is obvious from both Figures 62 and 63, facing failure because of tensile (or compressive) stresses would be one of the main failure modes. Other modes of failure for a panel under lateral loading are transverse shear failure of the core due to shear stresses, flexural crushing of the core because of plastic yielding or inelastic buckling of core struts and face wrinkling because of local buckling of the face sheet which is under compressive loads. The other modes are not the cases for this type of loading. For example, local crushing of the core (also known as indentation) occurs in case of concentrated load and is usually the case in three point bending as illustrated in Figure 
63. General buckling is not of the concern, since it only happens when the panel is under axial compressive loads. Thus, shear crimpling, which will occur as due to global buckling, is not going to happen. Finally, Intracellular dimpling is more common in honeycombs under compressive loads and is not important here.

It was already discussed that the failure modes have to investigated at either mesoscale or macroscales. In fact, face plastic failure, face wrinkling and transverse shear failure of the core must be studied in macroscale level because they should be dealt in much larger scales (whole panel size) than a lattice strut. However, the core crushing is known to be a mesoscale failure, as is involved with local plastic failure or inelastic buckling of the lattice struts.

In the following sections, these failure modes will be reviewed. 


\section{Facing failure}

Initial failure may occur in either compression or tension face. Caused by insufficient panel thickness, facing thickness or facing strength.

\section{Transverse shear failure} Caused by insufficient core shear strength or panel thickness.

\section{Flexural crushing of core}

Caused by insufficient core flatwise compressive strength or excessive beam deflection.

\section{Local crushing of core}

Caused by low core compression strength.

\section{General buckling}

Caused by insufficient panel thickness or insufficient core shear rigidity.

\section{Shear crimping}

Sometimes occurs following, and as a consequence of, general buckling. Caused by low core shear modulus or low adhesive shear strength.

\section{Face wrinkling}

Facing buckles as a plate on an elastic foundation. It may buckle inward or outward, depending on relative strengths of core in compression and adhesive in flatwise tension.
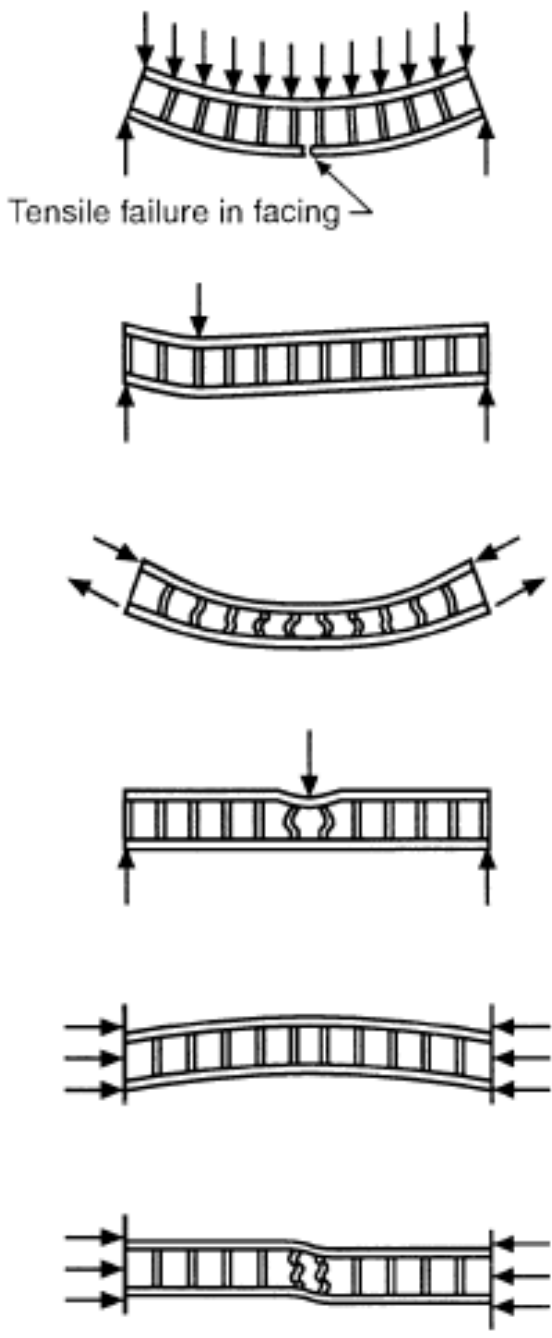

Adhesive bond failure
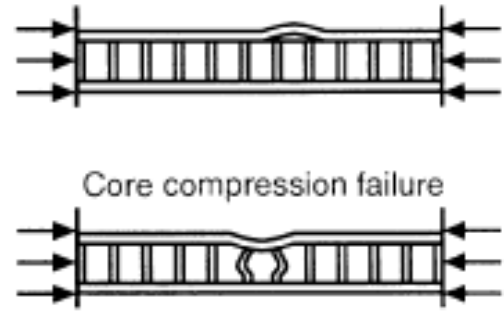

8. Intracell buckling (dimpling) Applicable to cellular cores only. Occurs with very thin facings and large core cells. This effect may cause failure by propagating across adjacent cells, thus inducing face wrinkling.

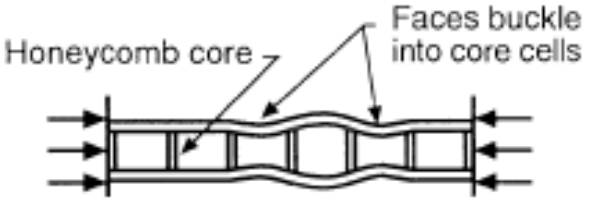

Figure 63: Sandwich failure modes [72] 


\subsection{Mesoscale Design}

The unit cell of an octet-truss lattice is shown in Figure 64. It is composed of a central octahedral cell constructed with 12 struts that is bounded by 8 edge tetrahedrons [68]. The cell has a Face Center Cube (FCC) lattice structure with cubic symmetry generating a material with an isotropic behavior [70]. It is assumed that the material used in fabrication of the lattice is elastic-perfectly plastic and has an isotropic behavior [70]. As was explained in Chapter 3, the minor anisotropic behavior of third generation aluminum-lithium alloys is ignored, and only their weakest direction is considered in design calculations. Since the variation in their mechanical properties is not much higher than other cold-worked isotropic metals, it makes sense to follow this approach for the sake of analysis.

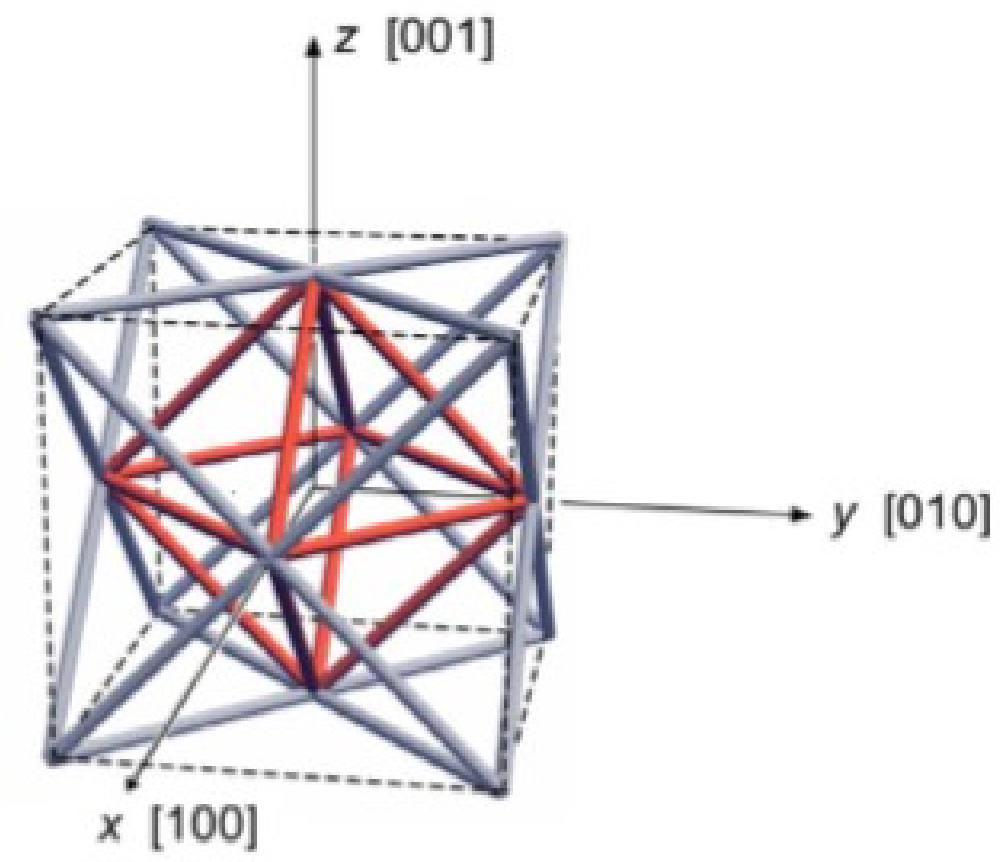

Figure 64: The unit cell of an octet-truss lattice [68] 


\subsubsection{Relative Density}

The relative density for an octet-truss lattice with vanishing node size, assuming the mass is equally distributed among the neighboring cells, can be expressed as [70]:

$\rho_{r}=\frac{\rho_{l}}{\rho_{0}}=6 \pi \sqrt{2}\left(\frac{r}{l}\right)^{2}$

Where $r$ is the radius of the cell strut cross-section, $l$ is the length of struts, $\rho_{r}$ is the relative density, $\rho_{l}$ is the density of the lattice material and $\rho_{0}$ is the density of the solid (base) material used to fabricate the lattice.

\subsubsection{Elastic properties}

As was already mentioned, the regular octet-truss cell geometry has cubic symmetry which generates a material with isotropic properties $[69,70]$. The linear elastic stress versus strain relationship for an isotropic material is [73]:

$$
[\varepsilon]=[\mathrm{C}][\sigma] \Rightarrow\left[\begin{array}{c}
\varepsilon_{1} \\
\varepsilon_{2} \\
\varepsilon_{3} \\
\varepsilon_{4} \\
\varepsilon_{5} \\
\varepsilon_{6}
\end{array}\right]=\left[\begin{array}{cccccc}
C_{11} & -C_{12} & -C_{13} & 0 & 0 & 0 \\
-C_{21} & C_{22} & -C_{23} & 0 & 0 & 0 \\
-C_{31} & -C_{32} & C_{33} & 0 & 0 & 0 \\
0 & 0 & 0 & C_{44} & 0 & 0 \\
0 & 0 & 0 & 0 & C_{55} & 0 \\
0 & 0 & 0 & 0 & 0 & C_{66}
\end{array}\right]\left[\begin{array}{c}
\sigma_{1} \\
\sigma_{2} \\
\sigma_{3} \\
\sigma_{4} \\
\sigma_{5} \\
\sigma_{6}
\end{array}\right]
$$

Where $[\varepsilon],[C],[\sigma]$ are strain, compliance and stress matrices, respectively. The compliance matrix is a symmetric matrix and can be simplified for an isotropic material by considering: $C_{11}=C_{22}=C_{33}=\frac{1}{E}, C_{12}=C_{13}=C_{23}=-\frac{v}{E}$ and $C_{44}=C_{55}=C_{66}=\frac{1}{G}$, where $E, G$ and $v$ are elastic modulus, shear modulus and Poisson's ratio of an isotropic material, respectively. The $[C]$ members can be simply derived by using basic strength of material principles, but those steps are skipped here. More information on deriving a compliance matrix is discussed by J.R Vinson (1999) [74]. The compliance matrix of an isotropic lattice material can be expressed as [70]: 
$[C]=\frac{1}{E_{l}}\left[\begin{array}{cccccc}1 & -v_{l} & -v_{l} & 0 & 0 & 0 \\ -v_{l} & 1 & -v_{l} & 0 & 0 & 0 \\ -v_{l} & -v_{l} & 1 & 0 & 0 & 0 \\ 0 & 0 & 0 & 2\left(1+v_{l}\right) & 0 & 0 \\ 0 & 0 & 0 & 0 & 2\left(1+v_{l}\right) & 0 \\ 0 & 0 & 0 & 0 & 0 & 2\left(1+v_{l}\right)\end{array}\right]$

Where $v_{l}$ and $E_{l}$ are the material Poisson's ratio, Young's modulus, respectively. The strain and stress matrices are defined as:

$\left[\begin{array}{c}\varepsilon_{1}=\varepsilon_{x x} \\ \varepsilon_{2}=\varepsilon_{y y} \\ \varepsilon_{3}=\varepsilon_{z z} \\ \varepsilon_{4}=\gamma_{y z}=2 \varepsilon_{y z} \\ \varepsilon_{5}=\gamma_{x z}=2 \varepsilon_{x z} \\ \varepsilon_{6}=\gamma_{x y}=2 \varepsilon_{x y}\end{array}\right]$ and $\left[\begin{array}{c}\sigma_{1}=\sigma_{x x} \\ \sigma_{2}=\sigma_{y y} \\ \sigma_{3}=\sigma_{z z} \\ \sigma_{4}=\sigma_{y z} \\ \sigma_{5}=\sigma_{x z} \\ \sigma_{6}=\sigma_{x y}\end{array}\right]$

The shear strain can be defined as: $\varepsilon_{i j}=\frac{1}{2}\left(\gamma_{i j}\right) ;(i$ and $j=x, y, z)$. Where $\gamma_{i j}$ is engineering shear strain. This is the definition used in many literatures. Figure 65 better illustrates the concept.

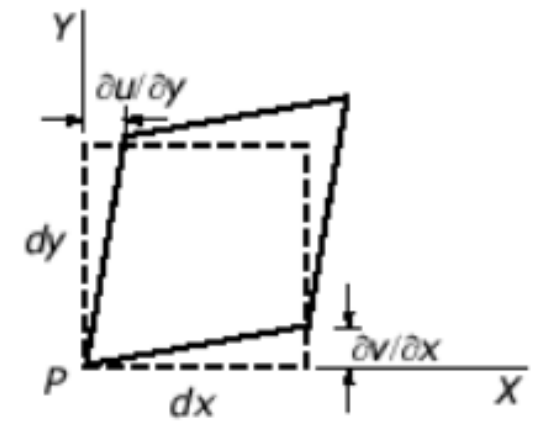

Shear strain is the average of two strains: $\varepsilon_{x y}=\left(\frac{\partial v}{\partial x}+\frac{\partial u}{\partial y}\right) \frac{1}{2}=\varepsilon_{y x}$

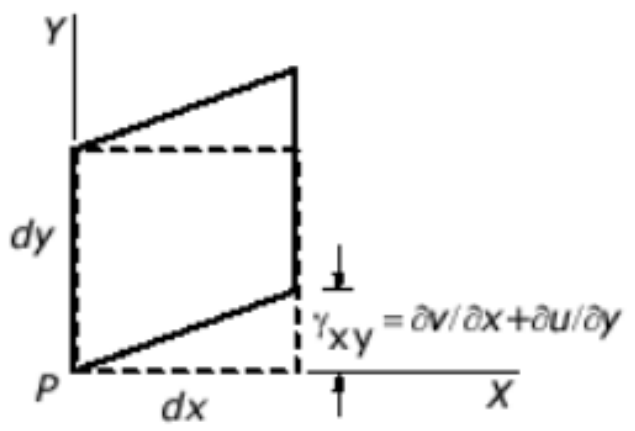

Engineer shear strain is the total shear strains: $\gamma_{x y}=\frac{\partial v}{\partial x}+\frac{\partial u}{\partial y}=\gamma_{y x}$

Figure 65: Engineering shear stress versus average shear stress [75] 
For example: $\varepsilon_{x y}=\left(\frac{\partial v}{\partial x}+\frac{\partial u}{\partial y}\right) \frac{1}{2}=\frac{1}{2} \gamma_{x y}=\frac{1}{2} \frac{\sigma_{x y}}{G_{x y}}$; This can be written as either $2 \varepsilon_{x y}=\left(\frac{1}{G_{x y}}\right) \sigma_{x y}$ or $\varepsilon_{x y}=\left(\frac{1}{2 G_{x y}}\right) \sigma_{x y}$. Where $G_{x y}$ is the shear modulus in x-y plane but there is no anisotropy in mechanical properties for this problem (Note: $\left.G=\frac{E}{2(1+v)}\right)$. As a result, the equation (4-3) can also be written as:

$\left[\begin{array}{c}\varepsilon_{x x} \\ \varepsilon_{y y} \\ \varepsilon_{z z} \\ 2 \varepsilon_{y z} \\ 2 \varepsilon_{x z} \\ 2 \varepsilon_{x y}\end{array}\right]=\frac{1}{E_{l}}\left[\begin{array}{cccccc}1 & -v_{l} & -v_{l} & 0 & 0 & 0 \\ -v_{l} & 1 & -v_{l} & 0 & 0 & 0 \\ -v_{l} & -v_{l} & 1 & 0 & 0 & 0 \\ 0 & 0 & 0 & 2\left(1+v_{l}\right) & 0 & 0 \\ 0 & 0 & 0 & 0 & 2\left(1+v_{l}\right) & 0 \\ 0 & 0 & 0 & 0 & 0 & 2\left(1+v_{l}\right)\end{array}\right] \cdot\left[\begin{array}{c}\sigma_{x x} \\ \sigma_{y y} \\ \sigma_{z z} \\ \sigma_{y z} \\ \sigma_{x z} \\ \sigma_{x y}\end{array}\right]$

Or

$\left[\begin{array}{c}\varepsilon_{x x} \\ \varepsilon_{y y} \\ \varepsilon_{z z} \\ \varepsilon_{y z} \\ \varepsilon_{x z} \\ \varepsilon_{x y}\end{array}\right]=\frac{1}{E_{l}}\left[\begin{array}{cccccc}1 & -v_{l} & -v_{l} & 0 & 0 & 0 \\ -v_{l} & 1 & -v_{l} & 0 & 0 & 0 \\ -v_{l} & -v_{l} & 1 & 0 & 0 & 0 \\ 0 & 0 & 0 & \left(1+v_{l}\right) & 0 & 0 \\ 0 & 0 & 0 & 0 & \left(1+v_{l}\right) & 0 \\ 0 & 0 & 0 & 0 & 0 & \left(1+v_{l}\right)\end{array}\right] \cdot\left[\begin{array}{c}\sigma_{x x} \\ \sigma_{y y} \\ \sigma_{z z} \\ \sigma_{y z} \\ \sigma_{x z} \\ \sigma_{x y}\end{array}\right]$

Therefore, depending on how the strain matrix is defined, the compliance matrix can be expressed as it was in equation (4-7), i.e. [C], or it can be expressed as it was in equation (4-8), i.e. [A].

$[\mathrm{C}]=\frac{1}{E_{l}}\left[\begin{array}{cccccc}\frac{1}{E_{l}} & \frac{-v_{l}}{E_{l}} & \frac{-v_{l}}{E_{l}} & 0 & 0 & 0 \\ \frac{-v_{l}}{E_{l}} & \frac{1}{E_{l}} & \frac{-v_{l}}{E_{l}} & 0 & 0 & 0 \\ \frac{-v_{l}}{E_{l}} & \frac{-v_{l}}{E_{l}} & \frac{1}{E_{l}} & 0 & 0 & 0 \\ 0 & 0 & 0 & \frac{1}{G_{l}} & 0 & 0 \\ 0 & 0 & 0 & 0 & \frac{1}{G_{l}} & 0 \\ 0 & 0 & 0 & 0 & 0 & \frac{1}{G_{l}}\end{array}\right]$ 


$$
[\mathrm{A}]=\left[\begin{array}{cccccc}
\frac{1}{E_{l}} & \frac{-v_{l}}{E_{l}} & \frac{-v_{l}}{E_{l}} & 0 & 0 & 0 \\
\frac{-v_{l}}{E_{l}} & \frac{1}{E_{l}} & \frac{-v_{l}}{E_{l}} & 0 & 0 & 0 \\
\frac{-v_{l}}{E_{l}} & \frac{-v_{l}}{E_{l}} & \frac{1}{E_{l}} & 0 & 0 & 0 \\
0 & 0 & 0 & \frac{1}{2 G_{l}} & 0 & 0 \\
0 & 0 & 0 & 0 & \frac{1}{2 G_{l}} & 0 \\
0 & 0 & 0 & 0 & 0 & \frac{1}{2 G_{l}}
\end{array}\right]
$$

Thus, one must be careful when deriving the compliance matrix, as both forms are acceptable.

The relative young's modulus $\left(E_{r}\right)$ of the octet-truss lattice can be expressed as [70]:

$E_{r}=\frac{E_{l}}{E_{o}}=\frac{2 \pi \sqrt{2}}{3}\left(\frac{r}{l}\right)^{2}$

Where $E_{o}$ is the Young's modulus of the solid material. From equations (4-3) and (4-11) it is concluded that [70]:

$E_{r}=\frac{E_{l}}{E_{o}}=\left(\frac{1}{9}\right) \rho_{r}$

The poison's ratio of the octet-truss lattice is found to be $v_{l}=1 / 3$ [70]. Thus, the compliance matrix can be written as [68]:

$[C]=\frac{1}{\rho_{r} \cdot E_{o}}\left[\begin{array}{cccccc}9 & -3 & -3 & 0 & 0 & 0 \\ -3 & 9 & -3 & 0 & 0 & 0 \\ -3 & -3 & 9 & 0 & 0 & 0 \\ 0 & 0 & 0 & 24 & 0 & 0 \\ 0 & 0 & 0 & 0 & 24 & 0 \\ 0 & 0 & 0 & 0 & 0 & 24\end{array}\right]$

Comparing equation (4-9) with (4-13) results in:

$G_{r}=\frac{G_{l}}{E_{o}}=\left(\frac{1}{24}\right) \rho_{r}$ 


\subsubsection{Strength properties}

\section{A-Plastic Yield Strength}

By using direct stiffness method, the relative axial and shear yield strength of the regular octet-truss lattice are obtained as $[70,73]$ :

$$
\begin{gathered}
\sigma_{r y}=\frac{\sigma_{y l}}{\sigma_{y o}}=2 \sqrt{2} \pi\left(\frac{r}{l}\right)^{2} \\
\tau_{r y}=\frac{\tau_{y l}}{\tau_{y o}}=\sqrt{2} \pi\left(\frac{r}{l}\right)^{2}
\end{gathered}
$$

Where $\sigma_{y l}$ and $\sigma_{y o}$ are tensile yield strengths of the lattice and solid material, respectively. $\tau_{y l}$ and $\tau_{y o}$ are shear yield strength of the lattice and solid material, respectively. Combining (4-3) into (4-15) and (4-16) results in [73]:

$$
\begin{gathered}
\sigma_{r y}=\frac{\sigma_{y l}}{\sigma_{y o}}=\left(\frac{1}{3}\right) \rho_{r} \\
\tau_{r y}=\frac{\tau_{y l}}{\sigma_{y o}}=\left(\frac{1}{6}\right) \rho_{r}
\end{gathered}
$$

\section{B-Buckling Strength}

The Euler critical buckling load of an axially loaded member in compression is expressed as [70]:

$P_{c r}=k^{2} \pi^{2} \frac{E I}{l^{2}}$

where $E$ is the Young's modulus of the material, $I$ is the smallest second moment of area of the member cross-section, $l$ is the length of the member and $k$ is a factor that depends on the rotational stiffness of the member end boundaries. For a pin-jointed element where joint rotation is freely allowed, $k=1$. If the rotation is fully constrained by fixed boundary conditions, then $k=2$. In practice, the value of the factor $k$ is between 1 and 2. The octet-truss lattice material idealized as a pin-jointed micro-truss structure with 
$k=1$, which is a safe design assumption [70]. The critical buckling stress of the lattice strut expressed as:

$\sigma^{c r}=\frac{P_{c r}}{A}=\frac{\pi^{2}}{4} E_{o}\left(\frac{r}{l}\right)^{2}$

Where $\sigma^{c r}$ and $A$ are critical buckling stress of a solid strut and cross sectional area of the lattice struts, respectively. Following the common approach in the literature for finding the critical bucking stress of the lattice $[64,68], \sigma_{y o}$ in equation (4-17) has to be replaced by $\sigma^{c r}$ given in equation (4-20). This will result in critical elastic buckling stress of the octet-lattice material:

$\sigma_{l}^{c r}=\frac{\pi^{2}}{12} E_{o}\left(\frac{r}{l}\right)^{2} \rho_{r}$

Combining equation (4-3) with (4-21):

$\sigma_{l}^{c r}=\frac{\sqrt{2} \pi^{3}}{2} E_{o}\left(\frac{r}{l}\right)^{4}$

Finally, the relative critical buckling strength would be:

$\sigma_{r}^{c r}=\frac{\sigma_{l}^{c r}}{\sigma_{y o}}=\frac{\pi}{72 \sqrt{2}}\left(\frac{E_{o}}{\sigma_{y o}}\right) \rho_{r}^{2}$

\subsubsection{Microscopic Failure}

The octet-truss lattice is a stretch dominant lattice. No matter what type of macroscopic load is being applied to the structure, the cell elements of a stretch dominant lattice are essentially loaded in axial tension or compression [70]. In some literature, the inelastic buckling equation is also presented. This equation is very similar to equation (4.17). The only difference is that the elastic modulus $(E)$ has to be replaced by the tangent modulus $\left(E_{t}\right)$ [68]. However, it was assumed that the base material is elasticperfectly plastic material. This assumption leads to a zero value for tangent modulus, and thus, the only possible buckling mechanism is elastic buckling [68]. Even if such an 
assumption had not been made, the inelastic buckling would not have been of the concern because the focus is only on the failure mode which occurs first and is known that both elastic buckling and plastic yielding occurs prior to inelastic buckling.

As a result, the only microscopic modes of failure include plastic yielding in tension and elastic buckling or plastic failure in compression [70]. It is assumed that the base material has a same compression and tension yield strength. This will narrow the competing failure mechanisms down to elastic buckling and plastic failure. However, it has been shown that low density stretch dominated lattice materials always fail by elastic buckling even when the macroscopic load is tension [70]. In fact, the elements fail in elastic region even before reaching yield strength. This is far from optimum design as the material would fail before accomplishing its potential strength [70].

As is obvious from the equations in the previous section, the failure mechanisms mainly depend on slenderness ratio of cell members $(a / l)$ and mechanical properties of the base material. Since the base material is already selected, the lattice strength would only depend on lattice density (slenderness ratio). In fact, there is a critical value for the density that can prevent failure by buckling. This critical value can be found from the below condition [70]:

$\sigma_{r}^{c r} \geq \sigma_{r y}$

Replacing equations (4-17) and (4-23) into the above inequality results:

$\rho_{r} \geq\left(\frac{24 \sqrt{2}}{\pi}\right)\left(\frac{\sigma_{y o}}{E_{o}}\right)$

It is obvious that the buckling was prevented by selecting the relative density (slenderness ratio) above a certain limit. This usually leads to higher lattice densities. The more efficient approach to this problem is to use hollow struts. As was explained before, 
the cross sectional shape was not investigated in this research because of the higher fabrication costs of hollow struts. The common and easily manufacturable shapes for a lattice strut cross section known to be circular or rectangular. In this thesis, the circular cross section is studied but the procedure and governing equations are very similar to the octet lattice with rectangular cross section. Additional information on governing equations for an octet lattice with rectangular cross section is presented by Donge et al., $2015[68]$.

\subsubsection{Collapse Surfaces}

The obtained relative density represents the minimum value of slenderness required to avoid buckling failure but it cannot guarantee the prevention of plastic yielding. The collapse surface of lattice materials is different from the solid materials which are usually analyzed by Tresca or von Mises method [70]. In fact, there is a set of equations that can be used for defining the collapse surfaces of a regular octet-truss lattice. Knowing that the failure mechanism is governed by plastic yielding, and the material has a same tensile and compression yield strength, the collapse surface in $x-y$ plane can be formulated as $[70,73]$ :

$$
\begin{aligned}
& \left|\frac{\sigma_{x x}}{\sigma_{y o}}\right|+\left|\frac{\sigma_{y y}}{\sigma_{y o}}\right| \leq 2 \pi \sqrt{2}\left(\frac{r}{l}\right)^{2} \\
& \left|\frac{\sigma_{x x}}{2 \sigma_{y o}}\right|+\left|\frac{\tau_{x y}}{\sigma_{y o}}\right| \leq \pi \sqrt{2}\left(\frac{r}{l}\right)^{2}
\end{aligned}
$$

It has to be mentioned that the symmetry of the octet-truss lattice material, is such that the set of collapse surfaces $\left(\sigma_{m m}, \sigma_{n n}\right)$ are identical, where $m$ and $n$ represent any pair of the $\mathrm{x}, y$ or $\mathrm{z}$ directions [73]. It means that the inequality (4-26) can be used for any combinations of normal stresses but $x-y$ plane was chosen as an example because it is the most important plane in plate theory, especially in case of thin plate assumption. The 
same principle is also valid for the inequality (4-27), i.e. symmetry dictates that the set of collapse surfaces $\left(\sigma_{m m}, \tau_{m n}\right)$ are identical [73].

Using the equation (4-3) in the above inequalities results in [70]:

$\left|\frac{\sigma_{x x}}{\sigma_{y o}}\right|+\left|\frac{\sigma_{y y}}{\sigma_{y o}}\right| \leq\left(\frac{1}{3}\right) \rho_{r}$

$\left|\frac{\sigma_{x x}}{2 \sigma_{y o}}\right|+\left|\frac{\tau_{x y}}{\sigma_{y o}}\right| \leq\left(\frac{1}{6}\right) \rho_{r}$

\subsection{Design Procedure}

In the previous section, the micro-failure mechanism, its collapse surfaces and governing equations were discussed. The next step would be the macroscale design; however, in order to do the macroscale design, one needs to start with the minimum lattice density (from equation (4-25)) as an input for the core material density in large scale sandwich panel. Then, conduct the macroscale analysis and optimization in order to determine the minimum core and face sheets thicknesses, this will be further discussed in the coming section. This step is followed by a FE simulation of the sandwich panel with its pre-determined thickness in order to find the stress distribution. Finally, the resulting stresses on the core material and face sheets have to be checked with both microscale and macroscale collapse criterion to make sure that the selected lattice density and plate thicknesses can withstand the applied loads. If the condition is satisfied, then that would be the end of the design process. Otherwise, higher values for the lattice density and/or the core and face sheet thicknesses must be selected, and the above procedure has to be repeated until the collapse criterions are satisfied. The diagram of the design process is shown in Figure 66. 


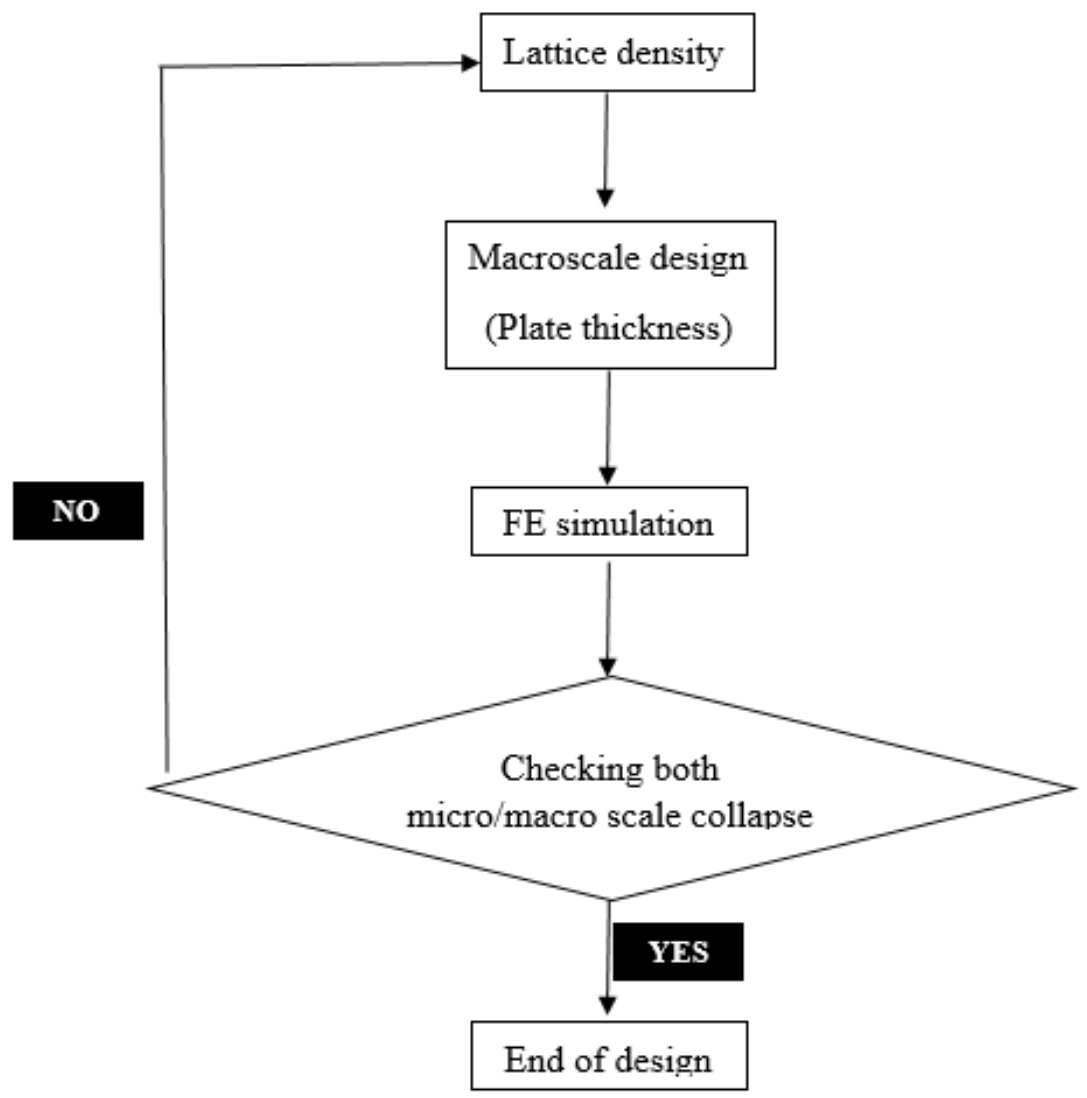

Figure 66: The schematic of the sandwich panel design process

In fact, this is an optimization loop which needs to be performed by a computer program to achieve the optimum result. However, many of the optimization algorithms need a starting point but no previous information is available for this design. Therefore, this research is focused on the basic design steps such as identification of the failure mechanisms, deriving governing equations, finding stiffness matrices, defining the design constraints and a preliminary structural optimization in order to come up with an initial design. Thus, following the proposed approach in many of the sandwich structure design guides, all design steps are performed manually (except the FEA analysis), and there would be no computer aided optimization at this preliminary design phase. 


\subsection{Macroscale Design}

\subsubsection{Modelling of Sub-Panels}

As was mentioned before, the focus of this research is on the hopper car floor panel with the loading conditions that were stated in Chapter 2. As a reminder, the floor panel is subjected to uniform lateral load (with its specific load factor). The longitudinal symmetric model was supported by two stiffeners, and thus, the whole floor panel should be supported by 4 stiffeners. These stiffeners divide the panel into 5 sub-panels with almost same aspect ratio. This is shown in Figure 67, where each sub-panel is labeled with a number and dashed lines $\mathrm{A}$ and $\mathrm{B}$ are, respectively, transvers and longitudinal symmetric lines.

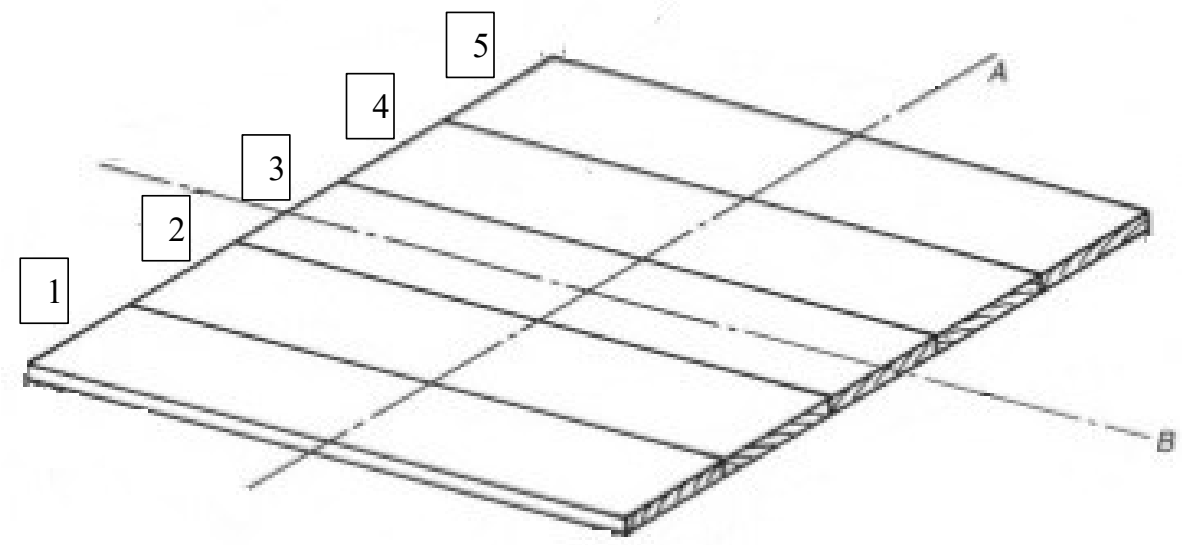

Figure 67: Schematic of the whole floor panel with its stiffeners [76]

Each of these stiffeners acts as a static support and implies a boundary condition (BC) on the panel that should be taken into account. Moreover, it is of the interest to compare FEA results with exact analytical solution to evaluate the proposed design. This is not possible when considering the whole panel with all its stiffeners, as there is no 
exact analytical solution available for that. Because of the mentioned reasons, only one of the subsections with its imposed BCs is selected for further analysis. Since the load is uniform on floor panel and all sub-panels have almost same dimensions and boundary conditions, the obtained results for any of the sub-panels (thickness, density and stiffness matrix) would be applicable for the other ones as well. However, if any differences are found in sub-panels that might affect the results, one can exactly follow the procedure described here to obtain the properties for any other sub-panels with different aspect ratio or boundary conditions. Here, the middle panel (number 3) is selected for submodeling. The sub-panel 3 itself can be shown as Figure 68. From CAD model in Chapter 2, $a=$ $3038 \mathrm{~mm}$ and $b=1290 \mathrm{~mm}$ for the center sub-panel.

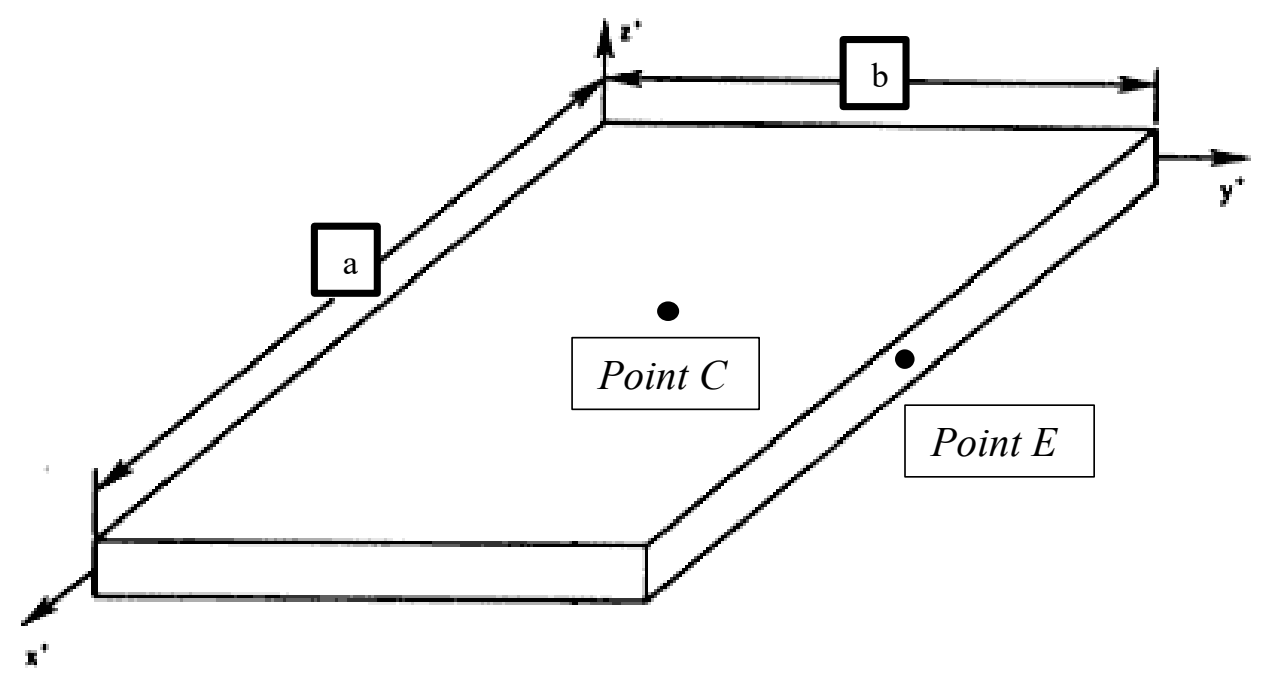

Figure 68: Schematic of the sample sub-panel [74]

The two opposite sides in the y-direction, are supported on stiffeners so their BC is very close to simply support type. The two ends in $\mathrm{x}$ direction, are usually welded to the side welds. The actual behavior at joints is in fact, something between clamped and 
simply supported type boundary conditions but it would be more similar to simply supported type where the rotation is allowed. As a result, the problem can be simplified to a rectangular panel with all edges simply supported under uniform lateral pressure. From Chapter 2, it is known that the actual pressure is $p_{o}=0.026 \mathrm{MPa}$ but with the AAR's load factor for the floor panel (1.8) [6], this would be almost $P_{o}=0.048 \mathrm{MPa}$.

\subsubsection{Stress Distribution and Analysis Method}

It was observed in Figure 62 that the maximum normal stress (tensile or compressive) occurs in the face sheets, while the maximum shear stress occurs in the core. It was also observed that the exact stress distribution diagram can be replaced by a more simplified model, called approximated model. For most sandwich applications, the difference between this approximation and the exact value is less than 1\% [77]. This is due to the fact that the approximated model is created based on two main assumptions that are usually valid for many sandwich structures. First, the core elastic stiffness is much smaller than of the face sheets $\left(E_{c} \ll E_{f}\right)$. Second, the thickness of the face sheets is much smaller than of the core material $\left(t_{f} \ll t_{c}\right)$. As a result, the face sheets can be considered as thin plates, assuming a constant normal stress distribution and linear shear stress distribution through their thickness, i.e. $\left(\frac{\partial \sigma}{\partial z}=0\right)$ and $\left(\frac{\partial \tau}{\partial z}=\right.$ const $)$. Moreover, the parabolic shear stress distribution in the core material is approximated with a constant shear stress through the core thickness. In some text books, the stress distribution is even more simplified to a degree that it is assumed there is no shear stress on the face sheets, and no normal stress on the core material.

Whether using the over-simplified model or the model shown in Figure 62, the main point is the face sheets carry the bending moments as tensile and compressive 
stresses and the core carries the transverse forces as shear stresses. A consequence is that for any sandwich construction, the deformation always consists of two parts: deformation due to bending and deformation due to shear [77]. There are different approaches for analyzing a rectangular plate like Roark's [78] or Timoshenko's [79] method but both of these methods neglect the shear deformations of the core, and therefore, are not exact solutions [72]. In some references like J.R Vinson (1999) [74], it is suggested to ignore the transverse shear deformation for a preliminary design but is also warned about the errors that this assumption could cause. Generally, the bending deflection equations can be found in many references but the shear deflection equations are harder to find [72]. However, the Composite Materials Handbook-17 (CMH-17) [77] provides equations that consider the transverse shear stresses and transverse shear deflection. The equations are based on the first-order shear deformation theory for thick plates [77]. While this theory it is compatible with plate-stress assumption, i.e. ignoring through thickness normal stresses, it considers a constant through thickness transverse shear stress which ultimately results in shear deformation. Derivation of the panel stress and deformation equations by considering the transverse shear deformation is a very complicated procedure and is not provided here but the final equations from the $\mathrm{CMH}-17$ approach [77] will be used for design of the panel.

\subsubsection{Failure Constraints}

The first step in design and optimization process is identification of the constraints on failure mechanisms [80]. It was explained in Section 4-7 that the macroscale failure mechanisms include face failure, face wrinkling and core transverse shear failure. In this section, all these failure mechanisms and their corresponding 
equations are investigated. These failure mechanisms ultimately define the constraints for the optimization problem.

It has to be reminded that the core material is isotropic, and the obtained results from microscale design now can be used as inputs for the lattice core material, using $c$ index instead of $l$. For example, $E_{l}=E_{c}, \sigma_{y l}=\sigma_{y c}, \rho_{l}=\rho_{c}$ and so on. The base material that the lattice core is made of is Al 2099, and it is the same material used for both face sheets. As a result, the properties of the base lattice material, shown with $o$ index, is same as face sheets' material, shown with $f$ index. For example, $E_{o}=E_{f}, \sigma_{y o}=\sigma_{y f}, \rho_{o}=\rho_{f}$ and so on. Due to the minor anisotropy in the selected material (Al 2099), the properties of the weaker direction are used. Using the data from Chapter 3, these properties are again summarized in Table 16.

Table 16: Material properties for face sheets and the base material that the lattice is made from [54]

\begin{tabular}{|c|c|c|c|c|c|}
\hline \multirow{2}{*}{ Material } & Density & Yield Strength & Modulus & Shear Modulus & Poisson's \\
& $\left(\mathbf{K g} / \mathbf{m}^{\mathbf{3}}\right)$ & $\mathbf{( M P a )}$ & $\mathbf{( G P a )}$ & (GPa) & Ratio \\
\hline Al 2099 & 2630 & 485 & 78 & 29 & 0.33 \\
\hline
\end{tabular}

\section{A- Face Failure}

Using the equations (4-12) and (4-17), the uniaxial yield strain of the core $\left(\varepsilon_{y c}=\right.$ $\left.\frac{\sigma_{y c}}{E_{c}}\right)$ is higher than of the face sheets $\left(\varepsilon_{y f}=\frac{\sigma_{y f}}{E_{f}}\right)$. In another word, $\varepsilon_{y c}=3 \varepsilon_{y f}$. Considering the continuity in displacement (strain) at the interface of the core and face sheets, it is concluded that the face sheet failure due to normal stresses occurs well before 
core failure. "Consequently, a constraint on face sheet yielding ensures that only shear yielding of the core need to be explicitly considered" [78].

The maximum bending stress on face sheets (stress at their centroid in the $b$ direction) is given by [77]:

$\sigma_{f, \max }= \pm k_{2} \frac{P_{o} \cdot b^{2}}{t_{f} \cdot d}$

Where \pm sign refers to the fact that one face is in tension and the other is in compression, $d$ is the distance between the face sheet centroids, i.e. $d=t_{f}+t_{c}$, and $k_{2}$ is a coefficient which only depends on the panel aspect ratio, if the core material is isotropic. Figure 69 presents a chart used for finding $k_{2}$. Considering the dimensions of $a$ and $b$ given in section 4.9.1 $(a / b=0.42)$, and an isotropic core $(\mathrm{R}=1)$, this coefficient would almost be: $k_{2}=0 \cdot 115$.

On the tensile side the maximum face stress $\left(\sigma_{f, \max }\right)$ should not exceed the face sheet yield strength but the situation is different on the compressed side. There are two competing failure mechanisms on the compressed side: plastic yielding and buckling (wrinkling). Since both tensile and compression yield strengths of the selected material are the same, it is clear that failure is restricted to either face sheet yielding $\left(\sigma_{y f}\right)$ or wrinkling $\left(\sigma_{w}\right)$, depending on the smaller of $\sigma_{w}$ and $\sigma_{y f}$ [80]. It means: $\sigma_{f, \max } \leq$ $\operatorname{Min}\left(\sigma_{y f}, \sigma_{w}\right)$. The face sheet wrinkling stress can be expressed as [81]:

$\sigma_{w}=0.5\left(E_{f} E_{c} G_{c}\right)^{1 / 3}$

It must be noted that different expressions have been developed for wrinkling stress but many of them overestimate the stresses. The above expression is widely used in industry for predicting wrinkling failure of panels loaded in bending or compression [81]. 
Using equation (4-12), inequality (4-25) and the data in Table 16, it is understood that: $\sigma_{y f}<\sigma_{w}$. Thus, the constraint on face failure would be:

$\sigma_{f, \max } \leq \sigma_{y f}$

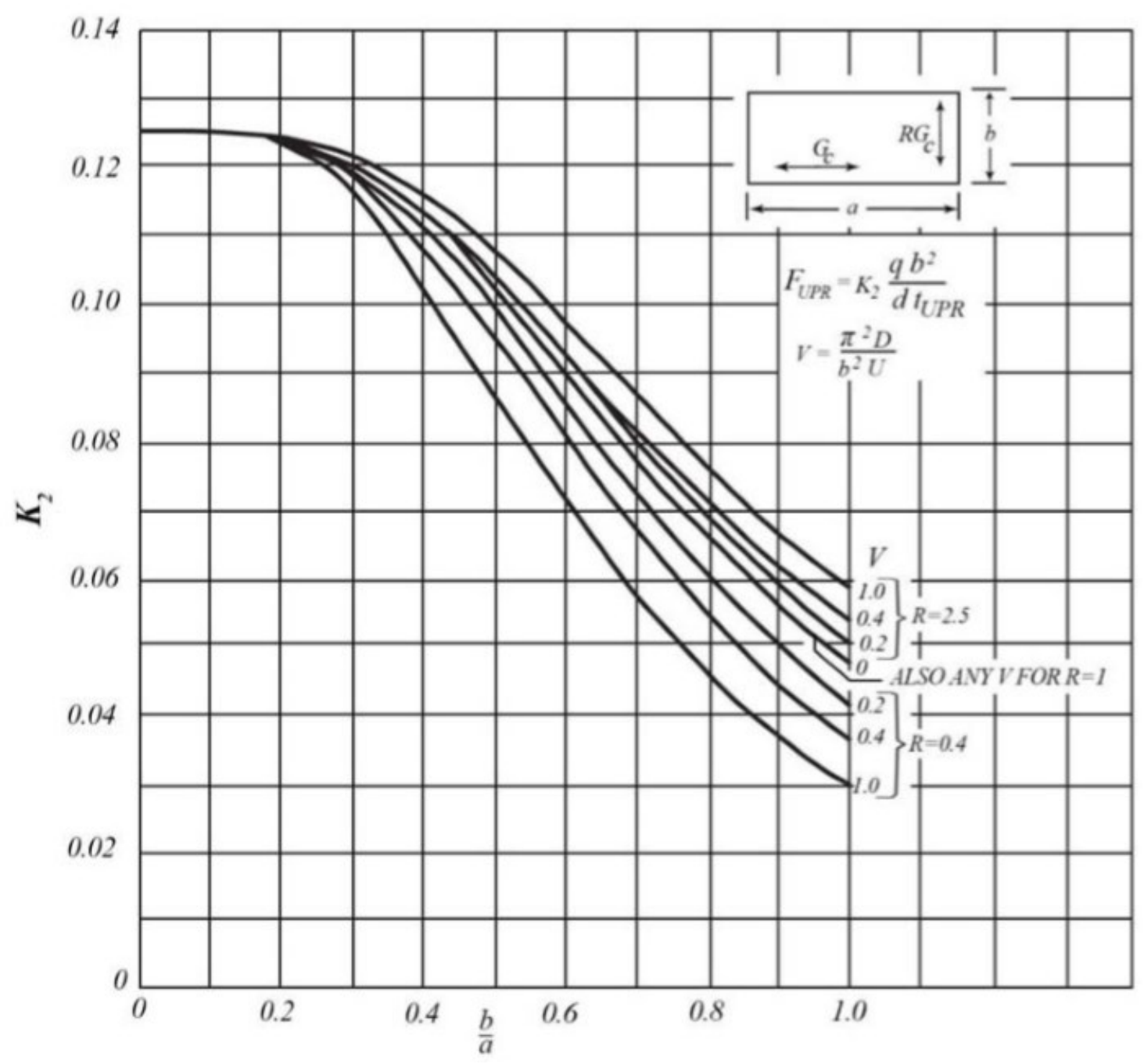

Figure 69: $K_{2}$ for determining face sheet stress in a flat rectangular sandwich panel with isotropic face sheets and isotropic core $(R=1)$ or orthotropic core $(R=0.4$ or 2.5$)$ core [77]

Same as in lattice struts, it is desired if the buckling failure (face wrinkling) occurs after the face yielding. This is structurally more optimal since the full strength 
potential of the material can be used, whereas in case of elastic wrinkling, the material fails in elastic region before fulfilling its yield strength. In the above calculations the relative density was 0.07 , which is almost the lower bound of the given spectrum from inequality (4-25). In fact, for relative densities above 0.02 , the face wrinkling stress exceeds the yield strength, and this is why it can be said that any random relative densities selected from inequality (4-25) results in $\sigma_{y f}<\sigma_{w}$. Indeed, in this specific case, the constraint on prevention of elastic buckling in lattice struts, inequality (4-25), can automatically satisfy the constraint on prevention of elastic buckling (wrinkling) on face sheets.

\section{B-Core Failure}

It was discussed that with a constraint on face plastic failure, it can be assured that the failure of the core is only due to transverse shear stress. The core shear stress is maximum at the panel edges, at mid-length of each edge [77]. The maximum core shear stress of the panel is given by [77]:

$\tau_{c, \max }=k_{3} \frac{P_{o} \cdot b}{d}$

Where $k_{3}$ is a theoretical coefficient dependent upon panel aspect ratio and the parameter $V$. If the core is isotropic, values of $V$ do not affect the core shear stress [77]. Figure 70 presents charts used for finding $k_{3}$ at edges of length $b$ and $a$ [77]. Considering $(R=1)$ for an isotropic core and the aspect ratio of $a / b=0.42$, the coefficient $k_{3}$ for edges $b$ and $a$ would be almost 0.37 and 0.48 , respectively. It is obvious that the maximum shear stress is higher on the longer edge (edge $a$ ), and thus, this would be the basis for the core design, i.e. $k_{3}=0.48$. The constraint on the core is:

$$
\tau_{c, \max } \leq \tau_{y c}
$$



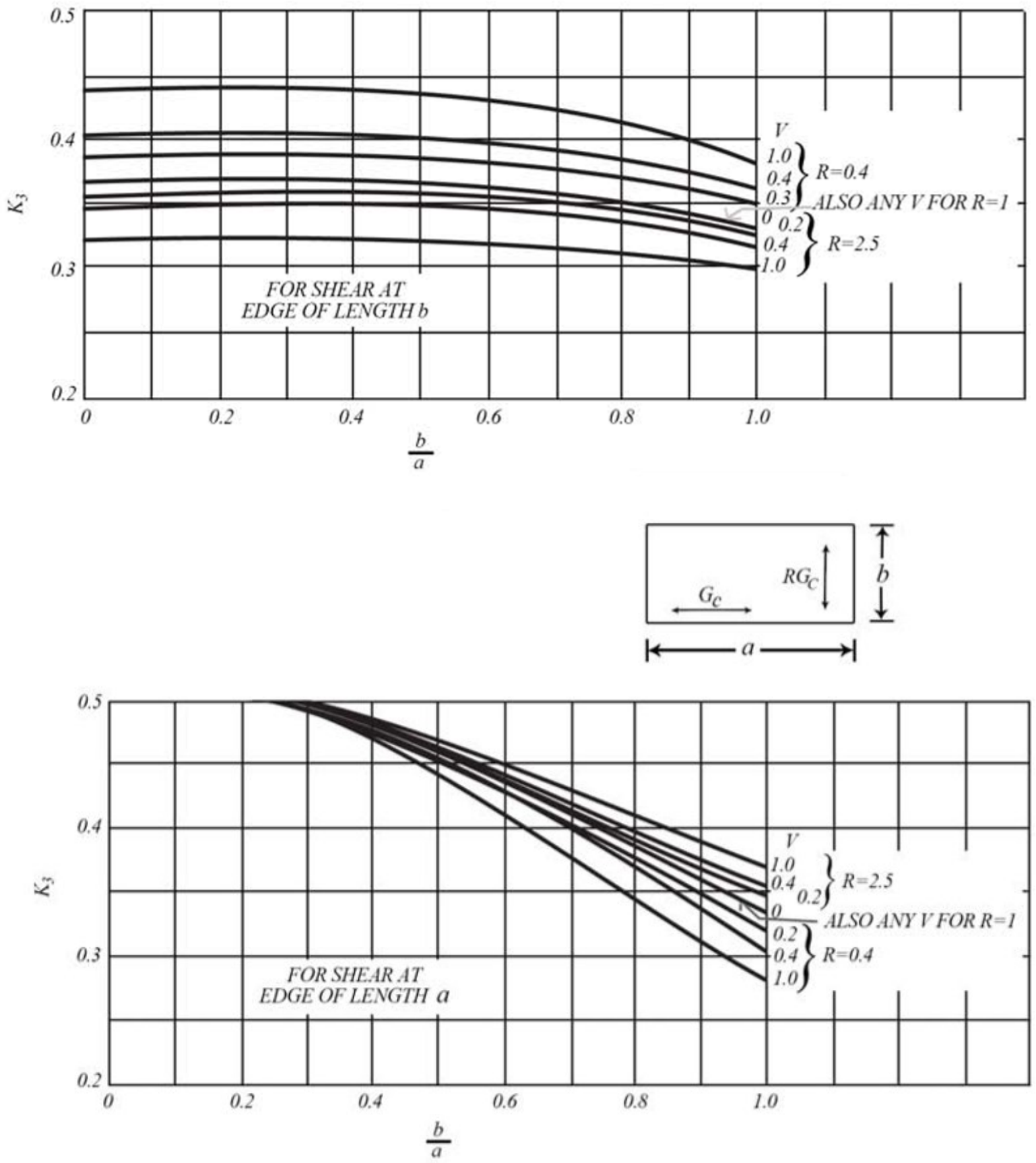

Figure 70: $\mathrm{K}_{3}$ for determining maximum core shear stress of a flat rectangular sandwich panel with isotropic face sheets and isotropic core $(R=1)$ or orthotropic core $(R=0.4$ or 2.5$)$ [77] 


\subsection{Weight Optimization}

\subsubsection{Geometrical Constraints}

In the previous section, the design constraints regarding the structural behavior of the sandwich structure were derived. However, there are also some geometrical constraints that must be defined for the optimization problem. The designer has more freedom in defining these type of constraints, since they are not as restricted as structural constraints. The geometrical constraints required for this design case are described below.

First, the thickness of the both face sheets together should not exceed the thickness of a monocoque panel traditionally used for hopper cars $(7 \mathrm{~mm})$ because all efforts for sandwich design would be meaningless if weight reduction is not achieved. Knowing the designed sandwich panel is a mid-plane symmetric panel, this constraint is given as:

$t_{f} \leq 3 \mathrm{~mm}$

Second, the ratio of the core thickness to the face thickness should comply with the assumptions stated for stress distribution. Although there is no fix value for this ratio, it is generally known that the stated assumptions are accurate if this ratio is less than $5 \%$. Here, this ratio is defined based on the distance between face sheet centroids, $d$. This means:

$t_{f} / d \leq 1 / 20$

Finally, the overall thickness of the sandwich panel should not exceed 25 mm. This constraint is defined to restrict the feasible design set and to prevent the selection of very large size panels which might affect the cargo capacity. This constraint can be written as: 
$d+t_{f} \leq 25 \mathrm{~mm}$

\subsubsection{Optimization Problem}

Ignoring the weight of bonding between the face sheets and the core material, the weight of the sandwich plate (in $K g$ ) can be expressed as:

$W_{s}=a b\left(2 \rho_{f} t_{f}+\rho_{c} t_{c}\right)$

Since the dimensions of the railcar panel and the densities of the core and face sheet materials are fixed, the above equation can be divided by $\left(a b \rho_{f}\right)$ and the resulting equation would be:

$w=2 t_{f}+\rho_{r} t_{c}$

Where $w$ is the goal function defined based on design variables $\left(t_{c}\right.$ and $\left.t_{f}\right)$. The aim is to minimize this function according to previously introduced design constraints, i.e. all geometrical constraints, constraint on face and core failure. Using equation (4-30), the constraint on face failure, inequality (4-32), can be expanded as:

$t_{f} \cdot d \geq k_{2} \frac{P_{o} \cdot b^{2}}{\sigma_{y o}}$

Using equations (4-18) and (4-33), the constraint on face failure, inequality (434), can be expanded as:

$d \geq 6 k_{3} \frac{P_{o} b}{\rho_{r} \cdot \tau_{y o}}$

Finally, by inserting the given values for $k_{2}, k_{3}, P_{o}, b, \rho_{r}$ and $\sigma_{y o}$ the optimization problem can be formulated. Although the main function is defined based on $t_{f}$ and $t_{c}$, the constraints are defined based on $d$. But that will not cause any difficulties, since $d$ itself can be defined in terms of $t_{f}$ and $t_{c}\left(d=t_{c}+t_{f}\right)$. The optimization problem is give as: 
Minimize: $w_{\left(t_{f}, t_{c}\right)}=2 t_{f}+0.07 t_{c}$

\section{Subjected to:}

$$
\begin{aligned}
& g_{1}=18.94-t_{f} \cdot d \leq 0 \\
& g_{2}=10.5-d \leq 0 \\
& g_{3}=\frac{1}{20}-\frac{t_{f}}{d} \leq 0 \\
& g_{4}=d+t_{f}-25 \leq 0 \\
& g_{5}=t_{f}-3 \leq 0
\end{aligned}
$$

The above problem is a relatively simple optimization of a two-variable function.

Therefore, the problem can be solved by the graphical method and there is no need for complicated search algorithms. As was stated, the constraints could have also been defined in terms of $t_{f}$ and $t_{c}$ (instead of $d$ ) but this would be unnecessary. Constraint $g_{2}$ and $g_{5}$, represents the lower bound and upper bound for $d$ and $t_{c}$, respectively. It is also obvious that both $t_{c}$ and $d$ are positive values.

The graphical solution is shown in Figure 71, where the feasible set of each constraint is shown with the dashed line with the same color of its function. The final feasible set of the problem is where all these feasible sets coincide. This area is a trianglelike shape highlighted with thick black lines. These boundaries themselves are also considered as part of feasible set. The optimum point is a point with the smallest possible $t_{c}$ and $t_{f}$, meaning that it should be toward the left bottom side. But when it comes to the trade-off between $t_{c}$ and $t_{f}$, e.g. on $g_{1}$, it is desired to select points with smaller $t_{f}$ and bigger $t_{c}$ because the contribution of the core to the overall weight is much smaller than of the face sheets. In fact, it was observed that by moving toward higher $d$ values on $g_{1}$ 
graph, the goal function decreased, and thus, the optimum point would be: $p^{*}=\left(t_{f}=0.8\right.$, $d=24.2$ ), marked with a star sign. Using the relation between $d, t_{f}$ and $t_{c}$, it is concluded that: $t_{f}=0.8 \mathrm{~mm}$ and $t_{c}=23.4 \mathrm{~mm}$.

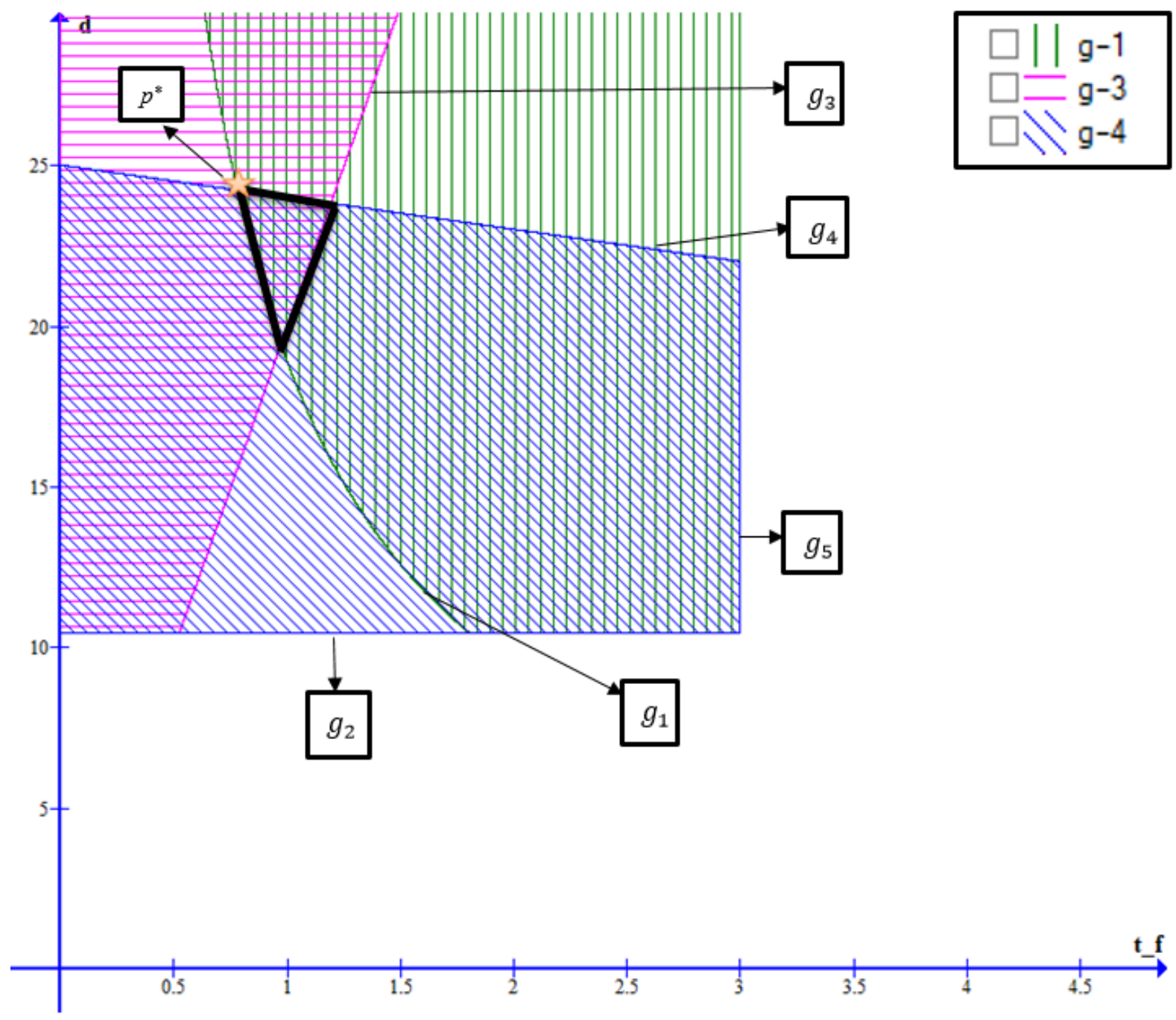

Figure 71: Schematic of the graphical optimization with the constraints and feasible set

The sandwich construction technique and following weight optimization can lead to a lighter structure but some challenges might arise as a result of using very thin face sheets. First, is the piercing of the face sheet when the railcar is being loaded. This may 
not an important issue when the railcar is loaded with sand but can be more challenging if the cargo is a metallic material. In that case, the denting (or piercing) can be prevented with adequate coating of the face sheets. This is a common approach which is currently being used in hopper cars and can be adopted for the proposed design as well. The other challenge is the possibility of the lower fatigue life of welds when the face sheets is attached to the other parts of the body. However, this was not considered, since the simulation of the welds are out of the scope of this research. But in a more detailed design of railcars, this can also be added as a constraint for the weight optimization problem.

In conclusion, the minimum core and face sheet thickness were derived in this chapter, but the obtained results have to be verified to make sure that the new optimized design will not fail. This will be done in the next chapter by comparing the FEA results with analytical results.

\subsection{Optimization Results Summary}

In this chapter a structural design method was proposed to achieve more weight reduction in the railcar body, while satisfying or even improving some of structural requirements. The proposed structural design known as sandwich type structure which typically consists of two strong face sheets and a relatively soft, low density core. The chapter started with emphasizing on advantageous and draw backs associated with this type of structures. It was followed by introducing the common structural core materials including honeycomb, lattice and foams. It was observed that the honeycomb and lattice materials outperform other structural cores but since honeycomb design has been well 
established in the literature and industry over last decades, it was decided to focus on truss-lattice core materials. For that, different lattice topologies were compared considering their manufacturability and mechanical properties, and the octet-truss lattice was selected for the design. In the following section, possible failure mechanisms in a sandwich type structure was studied and the common ones in panel under uniform load (bending) were introduced. Then, the appropriate failure mechanisms were investigated separately at two different levels, the mesoscale and macroscale. The information derived from mesoscale design (design of the lattice core) was taken as an input for the macroscale design. Finally, all these design constraints led to a weight optimization problem, where the minimum core and face sheet thicknesses were obtained.

The optimum design will be validated using analytical and FEA results in the next chapter. 


\section{Chapter 5. Validation}

In the previous chapter, the detailed design procedure for a rectangular sandwich plate under uniform lateral loading was discussed. The design was conducted in two different macroscopic and microscopic scales which led to constraints on the core relative density, core thickness and face sheets thickness. These design constraints were used in conjunction with a target function on the panel weight and formed an optimization problem. The solution to the optimization problem yielded the minimum values for design variables, where $\rho_{r}, t_{f}$ and $t_{c}$ are $0.07,0.8 \mathrm{~mm}$ and $23.4 \mathrm{~mm}$, respectively. However, in this chapter the optimized design has to be evaluated in the FE software, ABAQUS. This will start with an introduction to different types of FE modeling techniques for sandwich panels and be followed by comparing the results with the analytical results to make sure that the proposed design will not fail under the stated loading condition.

\subsection{Finite Element Modeling of Sandwich Structures}

This section is intended to provide some information on simulation of sandwich panels including element selection, different modeling techniques and their challenges. Some of the models can only represent the global response but on the other hand, there are some that can provide information on local responses as well. All techniques are accurate if implemented properly, and it is the user's desired output that defines which model could be the most suitable one. The important thing is the selected core elements should consider transverse shear deformation. This improves the accuracy of static analysis results but is much more crucial in case of buckling and/or modal analysis, as 
ignoring the shear defamation will lead to non-conservative results [77]. These modeling techniques include:

Global Model: In this method, the whole sandwich structure is replaced by a single-layer panel, and the equivalent stiffness matrix of the sandwich panel has to be used as an input for section properties. As is obvious from its name, this model represents the global behavior of the structure and does not include geometric details or local stresses. A common approach is to extract the element forces and moments from the global FE model results and use this information as input for further analysis to check the failure modes [77].

Layered (Stacked) Model: In this method, the sandwich panel is divided into three or more layers, and all layers have a common, unique the through cross section rotation [77]. Depending on the type of elements used for each layer, the method itself can fall into three categories: stacked shell, stacked shell/solid and stacked solid.

In the layered shell model, shell elements are used for all three layers. The elements used for core material must be shear-flexible which consider the transverse shear deformation, while the elements based on classical thin-plate theory can be used for the face sheets knowing that the transverse shear flexibility is being ignored in these types of elements [77]. Sometimes instead of adding layers, the model can be simulated by defining a composite layup in a single panel of the thickness equal to all layers. This type of simulation is known as composted layup in ABAQUS.

In the layered solid/shell model, the core and face sheets are simulated using solid and shell elements, respectively. This model can be used for both global and local response, and is computationally more expensive than stacked shell or global model. The 
challenge with this model is to select compatible solid and shell elements because some combinations of solid elements with shell elements may lead to incompatibility for the normal displacements [77].

The layered solid model is also known as full 3-D model, where 3D solid elements are used for both face sheets and the core [77]. This is not a common approach since the computational cost is the highest among other models. This technique can be implemented when local response at specific points are of the interest, e.g. in case of bolted joints or cracks.

Composite Shell: This model is very similar to the layered shell model but instead of adding layer upon layer, the method models the sandwich structure with the single laminated composite shell. This model is useful as both global response and stress distribution can be investigated.

Finally, it has to be mentioned that some software have special modules and elements for simulating sandwich structures. However, these elements are not widely available in commercial software and their formulations vary so the user should check the element documentation to understand what assumptions are used [77].

\subsection{Validation of Results}

Simulating the sandwich structures with 2-D models (elements) is a common practice because of their higher computational efficiency. In fact, both full 3D and solid/shell models are computationally expensive because they cannot properly simulate the deformation of a plate structure unless a highly refined mesh is being used through the thickness of the solid. In addition, the selection of compatible elements would also be 
a challenging issue. All these will increase the complexity of the problem and its computational time and is unnecessary if there are no stress concentrations in the structure like holes, bolts or cracks. Therefore, the approach would be toward 2D models. As was discussed, there are three different options for 2D simulation of a sandwich panel, i.e. global model, composite shell model and layered model (composite layup). All these models have their own strength and weaknesses. Here, all three options will be simulated using ABAQUS and their results will be compared with the analytical solutions to emphasize on their advantages and shortcomings. Further discussion on each model will be presented in the following sections.

All models are meshed with SR8 shell elements, which are second order quadrilateral shell elements and known as thick plate type elements. These elements are needed in cases where transverse shear flexibility is important and second-order interpolation is desired [29]. The S8R elements are used for the analysis of laminated composite shells and are based on first-order transverse shear flexible theory in which the transverse shear strain is assumed to be constant through the thickness of the shell [82]. In cases where the transverse shear defamation is important and thick plate simulation is desired, especially for the core, these elements are more suitable compared to general purpose shell elements, like S4R.

As was stated in Chapter 5, the maximum bending stress on face sheets occurs at their centroid in the $b$ direction [77], i.e. $\sigma_{y y}=\sigma_{22}$ at point $C$, see Figure 67. The equation for the maximum core shear stress at the mid-length of the large edge (edge $a$ ) was also given in Chapter 5 [77], i.e. $\tau_{y z}=\tau_{23}$ at point $E$, see Figure 67. The obtained $t_{f}$ and $t_{c}$ from the optimization problem will be used as inputs of equation (4-30) and (4- 
33), and the results will be compared with the FEA results. Moreover, although there is no requirement for allowable deflection in AAR [6], it is of the interest to compare the global response of the panel (maximum deflection) obtained from the FE modeling with the analytical results. The maximum deflection of the simply supported rectangular sandwich panel under uniform lateral loading is given by [77]:

$$
\delta_{\max }=2 k_{1}\left(1-v_{f}^{2}\right) \frac{p_{o} \cdot b^{4}}{E_{f} \cdot t_{f} \cdot d^{2}}
$$

Where $k_{1}$ is a coefficient dependent upon panel aspect ratio and the value of $V$ [77]. For a mid-plane symmetric sandwich panel this value is given by [77]:

$$
V=\frac{\pi^{2} \cdot t_{c} \cdot E_{f} \cdot t_{f}}{2\left(1-v_{f}^{2}\right) b^{2} \cdot G_{c}}
$$

The $k_{1}$ coefficient can be found in charts given in the CMH-17 [77] in the same manner that $k_{2}$ and $k_{3}$ were found in Chapter $4, k_{1}=0.012$. The analytical results versus the FE analysis is shown in Table 17, see Figure 67. It has to be noted that the convergence analysis was performed and the FE results are mesh independent. 
Table 17: Analytical results vs FE Analysis

\begin{tabular}{|c|c|c|c|}
\hline Parameter & $\begin{array}{c}\left(\boldsymbol{\sigma}_{\mathbf{2 2}}\right)_{\text {face }} \text { at } C \\
(\mathbf{M P a})\end{array}$ & $\begin{array}{c}\boldsymbol{\delta}_{\text {max }} \text { at } C \\
(\mathbf{m m})\end{array}$ & $\begin{array}{c}\left(\boldsymbol{\tau}_{\mathbf{2 3}}\right)_{\text {core }} \text { at } E \\
(\mathbf{M P a})\end{array}$ \\
\hline Analytical & 474.8 & 77.79 & $1.23 \mathrm{MPa}$ \\
\hline $\begin{array}{c}\text { FE Composite Shell } \\
(\% \text { difference) }\end{array}$ & 464.8 & 73.33 & $\mathrm{~N} / \mathrm{A}$ \\
\hline $\begin{array}{c}\text { FE Composite Layup } \\
(\% \text { difference) }\end{array}$ & $(-2.0 \%)$ & $(-5.7 \%)$ & $1.26 \mathrm{MPa}$ \\
\hline FE Global & N/A & 78.28 & N/A \\
$(\%$ difference) & & $(+0.6 \%)$ & \\
\hline
\end{tabular}

\subsubsection{Comparison of FE Models}

It is obvious that in terms of the global response $\left(\delta_{\max }\right)$, the global model illustrates the closest result to the analytical one with only $0.6 \%$ difference. However, it is not possible to study the stress distribution in this model. No material is defined in this model and only flexural, stiffness and coupling matrices need to be defined for this model. The detailed information on deriving these matrices for a sandwich panel is given in Jack R. Vinson (1999) [74].

The composite shell model represents the least difference from the analytical result in terms of maximum in-plane stress. The main advantage with this model is that both global response $\left(\delta_{\max }\right)$ and stress distribution can be presented using this model but the main problem is that it is not possible to investigate the local responses. In fact, the 
composite shell model represents the overall stress distribution for the whole model but cannot present the stress on each layer.

Finally, the layered model (composite layups) is more suitable for local responses. It cannot predict the global behavior $\left(\delta_{\max }\right)$ but can present the detailed information on each layer. Since it is of the interest to study the failure of each layer (face sheets and core), this model will be chosen over the other two options for failure analysis.

\subsubsection{Sources of Error}

There is a small difference between stresses on the face sheets $\left(\sigma_{22}\right)$ and core $\left(\tau_{23}\right)$ predicted by the FE models and analytical results. This difference is partly because of the inherent error in the FE method, and partly because of the error in reading the $k_{2}$ and $k_{3}$ coefficient from the charts. Of course, the inherent error is minimized by using a second-order element instead of a first-order element but still there is a difference between the actual function and the interpolated one. However, the difference is small for $\sigma_{22}$ in composite shell model and $\tau_{23}$ in the layered model which shows there is a good match between results. The error in $\sigma_{22}$ for the layered model is a little larger but still in an acceptable range considering even in some of ABAQUS case studies for sandwich panels the error in the stress reaches $6.4 \%$ depending on the selected element [83].

The global model is not discussed because of its very small error compared to analytical results but the error in deflection of the composite shell is studied. It is obvious that both inherent FEA error and human error in reading the $k_{1}$ coefficient affect the result but there is another factor involved with this in addition to those factors. The lower deflection predicted by the FEA is mainly because of the selection of thick-shell elements for both face sheets and core material. In fact, when the whole panel is simulated as a 
single composite shell, it is not possible to use different types of elements for different layers. On the other hand, the analytical equation only considers the core as a thick plate and treats the face sheets as thin plates, whereas the whole panel is simulated as a thick plate in the FE model. As a result, the FE model is stiffer and shows lower deflection. Nevertheless, the difference is not huge and still there is a good agreement between analytical and theoretical results noting that even in some of ABAQUS case studies these difference in deflection reaches $9.8 \%$ depending on the selected element [83].

\subsubsection{Failure Analysis}

It is of the interest to see if the face sheets and core material would fail under stated loading conditions or not. For the face sheets, like any other ductile material, the von Mises yield criterion can be used. The obtained equivalent von Misses stress from the composite shell and layered model are 404.2 $\mathrm{MPa}$ and $391.2 \mathrm{MPa}$, respectively. Whether to use the composite or layered model, their maximum von Misses stress would be lower than the face sheet yield strength $(485 \mathrm{MPa})$. Therefore, the new proposed design would not fail on its face sheets. The equivalent von Misses stress distribution for composite and layered model are shown in Figures 72 and 73, respectively. 


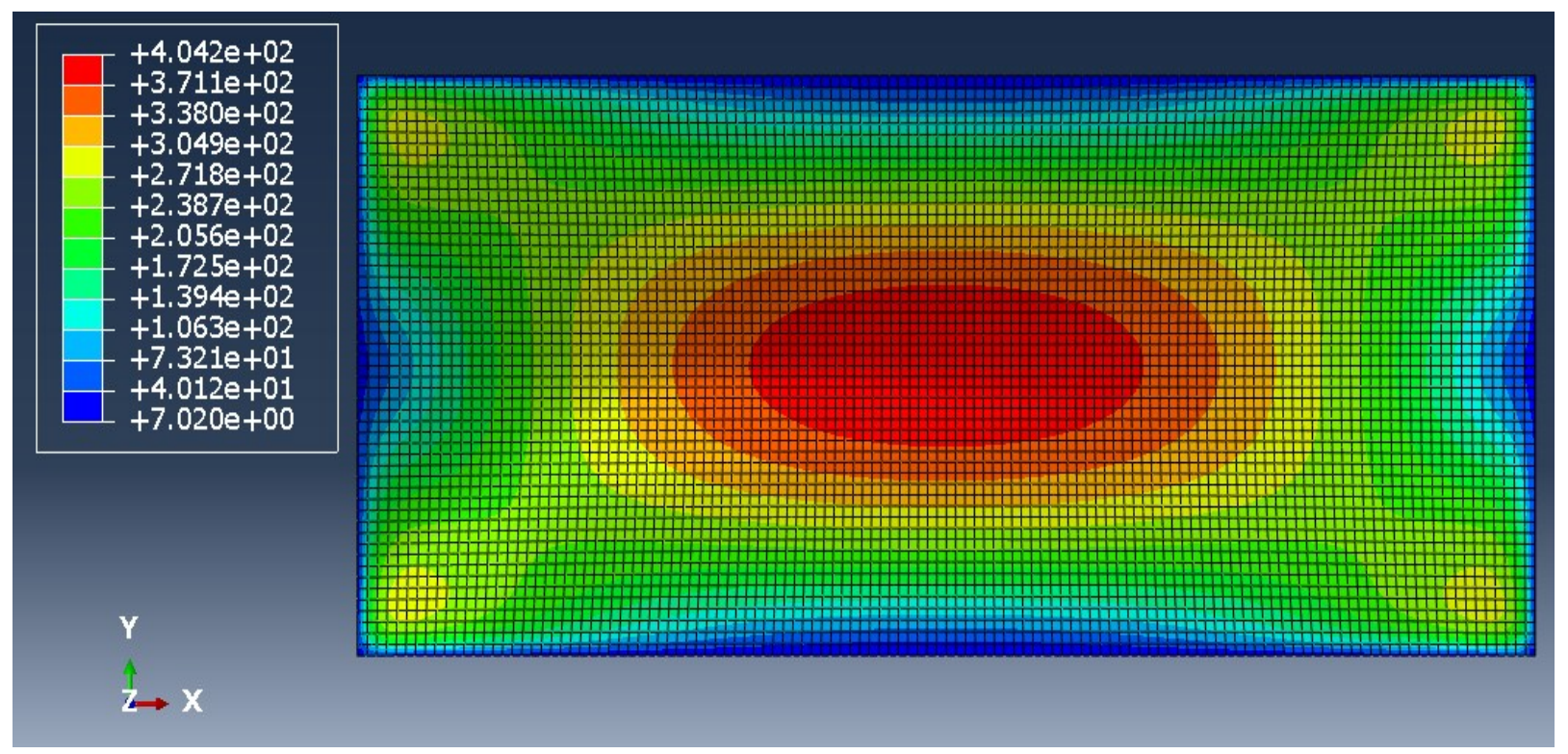

Figure 72: The equivalent von Mises stress (MPa) in the composite shell model (for the whole model)

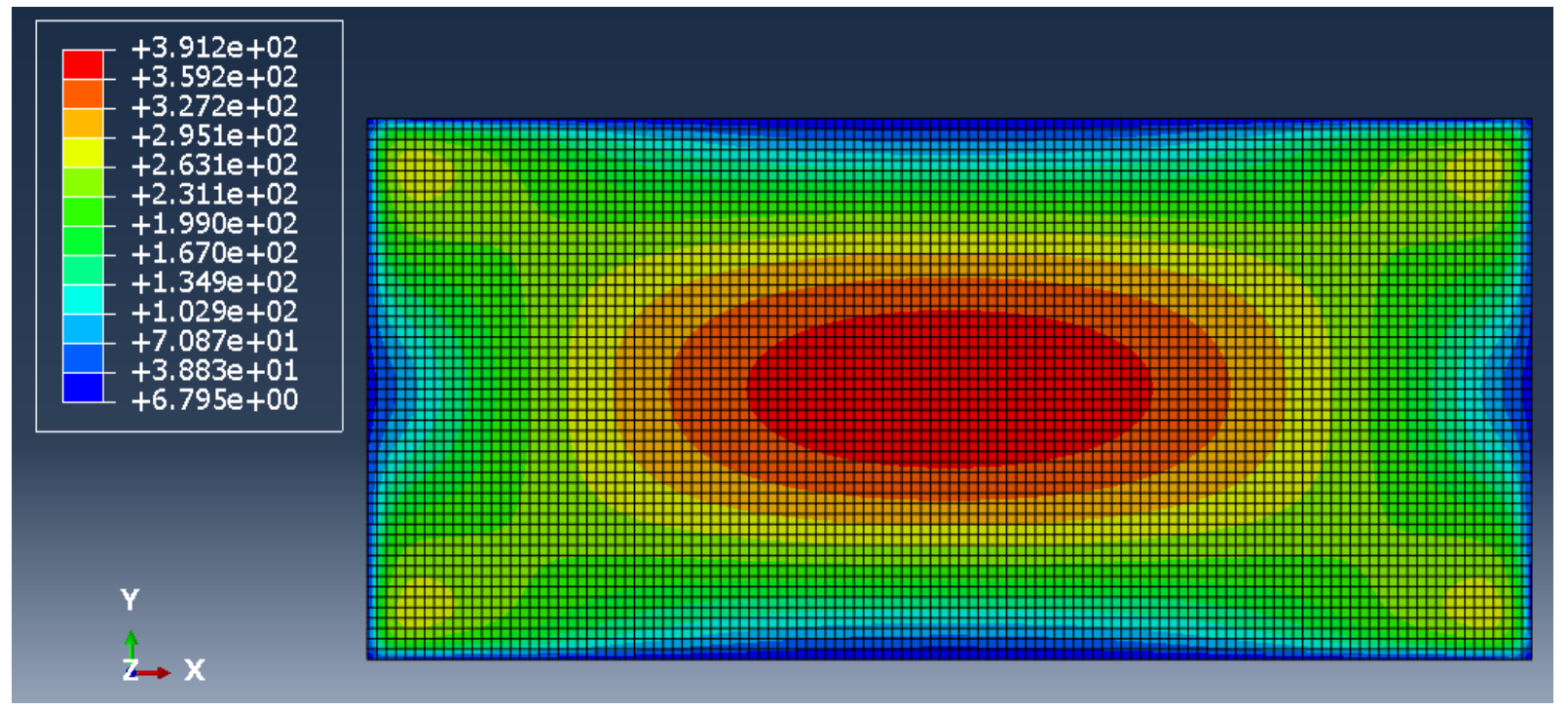

Figure 73: The equivalent von Mises stress (MPa) in the layered model (for the first layer: face sheet)

However, as discussed in Chapter 4, the failure criterion of the core material (lattice truss) is different from the ductile materials. The failure criterion was given in inequalities (4-28) and (4-29). This means that in a safe design, any combination of 
normal stresses should not exceed the limit specified in the inequality (4-28), that is: $\left(\frac{1}{3}\right) \rho_{r}=0.023$, and any combinations of the shear stresses with normal stresses should not exceed the limit specified in the inequality (4-29), that is: $\left(\frac{1}{6}\right) \rho_{r}=0.012$. This can be investigated by analyzing the stress distribution in the core material.

Since the plane-stress assumption is involved with this problem $\left(\sigma_{z}=0\right)$, the equation (4-28) will have only one form that was presented in Chapter 4. However, all the in-plane stresses including $\sigma_{x x}, \sigma_{y y}$ and $\tau_{x y}$ in the core material are very small. They all have a magnitude of $10^{-18} \mathrm{MPa}$ to $10^{-17} \mathrm{MPa}$, see Figures $74-76$, which can for sure satisfy the stated criterion in inequality (4-28).

Due to the very small magnitude of normal stress the first term in the inequality (4-29) can be ignored, i.e. $\left|\frac{\sigma_{x x}}{2 \sigma_{y o}}\right|=\left|\frac{\sigma_{y y}}{2 \sigma_{y o}}\right|=0$. Thus, the inequality (4-29) can be written in a general from as:

$\left|\frac{\tau_{m n}}{\sigma_{y o}}\right| \leq\left(\frac{1}{6}\right) \rho_{r}$

As was explained in Chapter 4, $m$ and $n$ represent any pair of the $\mathrm{x}, \mathrm{y}$ or $\mathrm{z}$ directions [73]. Again, because of the small in plane shear stress $\left(\tau_{x y}\right)$, the above inequality can have only two different forms in terms of $\tau_{y z}$ and $\tau_{x z}$. That is:

$$
\begin{aligned}
& \left|\frac{\tau_{y z}}{\sigma_{y o}}\right| \leq\left(\frac{1}{6}\right) \rho_{r} \\
& \left|\frac{\tau_{x z}}{\sigma_{y o}}\right| \leq\left(\frac{1}{6}\right) \rho_{r}
\end{aligned}
$$

In this design case, the above inequalities can be defined as the failure criterion for the lattice core. If the maximum shear stresses on the lattice core, $\left(\tau_{y z}\right)_{c, \text { max }}$ and $\left(\tau_{x z}\right)_{c, \text { max }}$, can satisfy the inequalities, there would be no failure in the core and design 
can be regarded as a safe design. The maximum shear stresses on the sandwich core are: $\left(\tau_{y z}\right)_{c, \max }=3.80 \mathrm{MPa}$ and $\left(\tau_{x z}\right)_{c . \max }=3.90 \mathrm{MPa}$. Since $\left(\tau_{x z}\right)_{c, \max }$ is bigger than $\left(\tau_{y z}\right)_{c, \text { max }}$, only the inequality (5-5) needs to be studied. Substituting the maximum shear stress in the inequality $(5-5)$ will result in: $0.008<0.012$, and this means that the sandwich design will also not fail on its core. Even if one is interested in checking the macroscopic failure analysis it can be seen that: $\tau_{c, \max }=3.90 \mathrm{MPa}<\tau_{y c}=5.66 \mathrm{MPa}$, where $\tau_{y c}$ is the shear yield strength of the core material and can be found using equation (4-18). To sum up, it is understood that the proposed design is a safe design both in microscopic and macroscopic level, and none of the face sheets and the core material would not fail under the load of cargo weight. The stress distribution on the core material is shown in Figures 74-78.

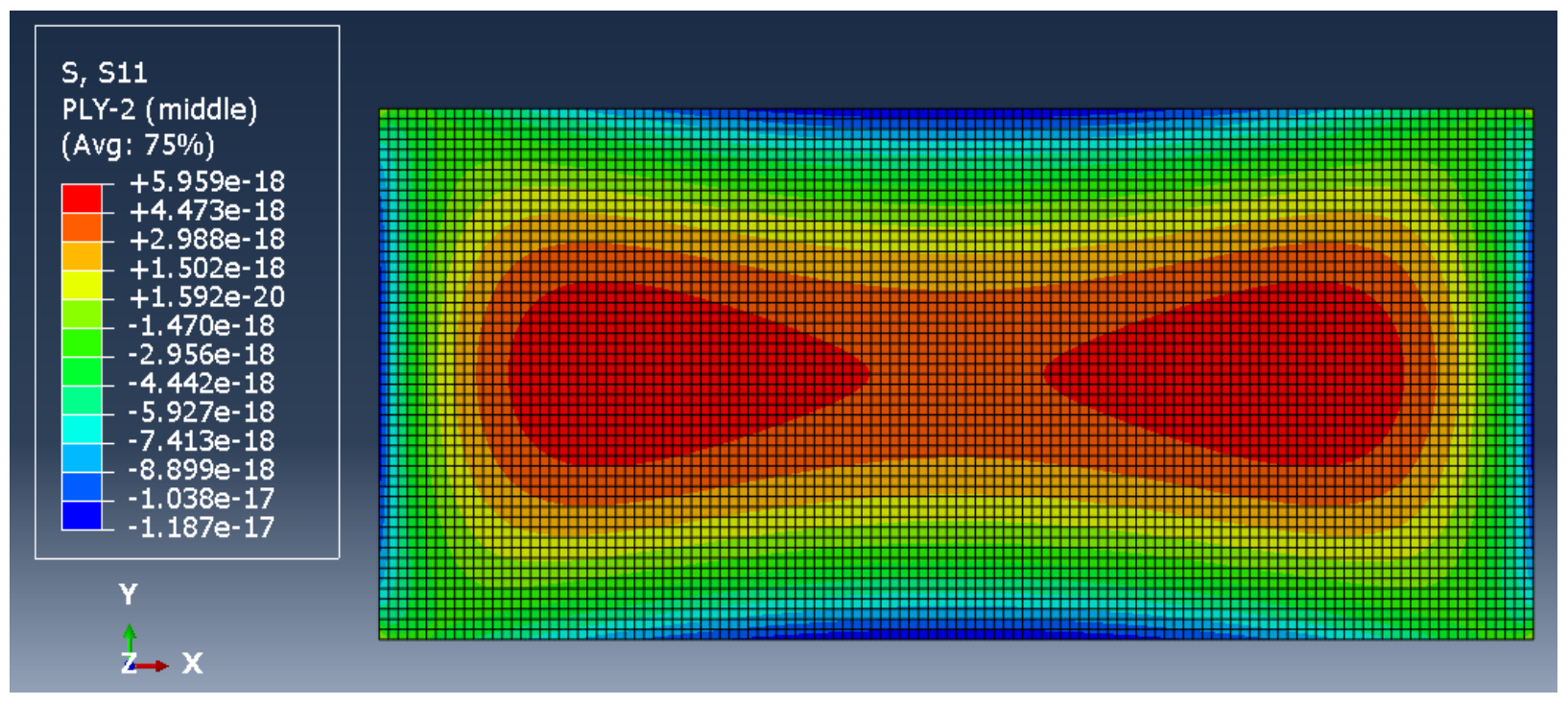

Figure 74: The in-plane normal stress, $\sigma_{x x}=\sigma_{11},(\mathrm{MPa})$ on the core material 


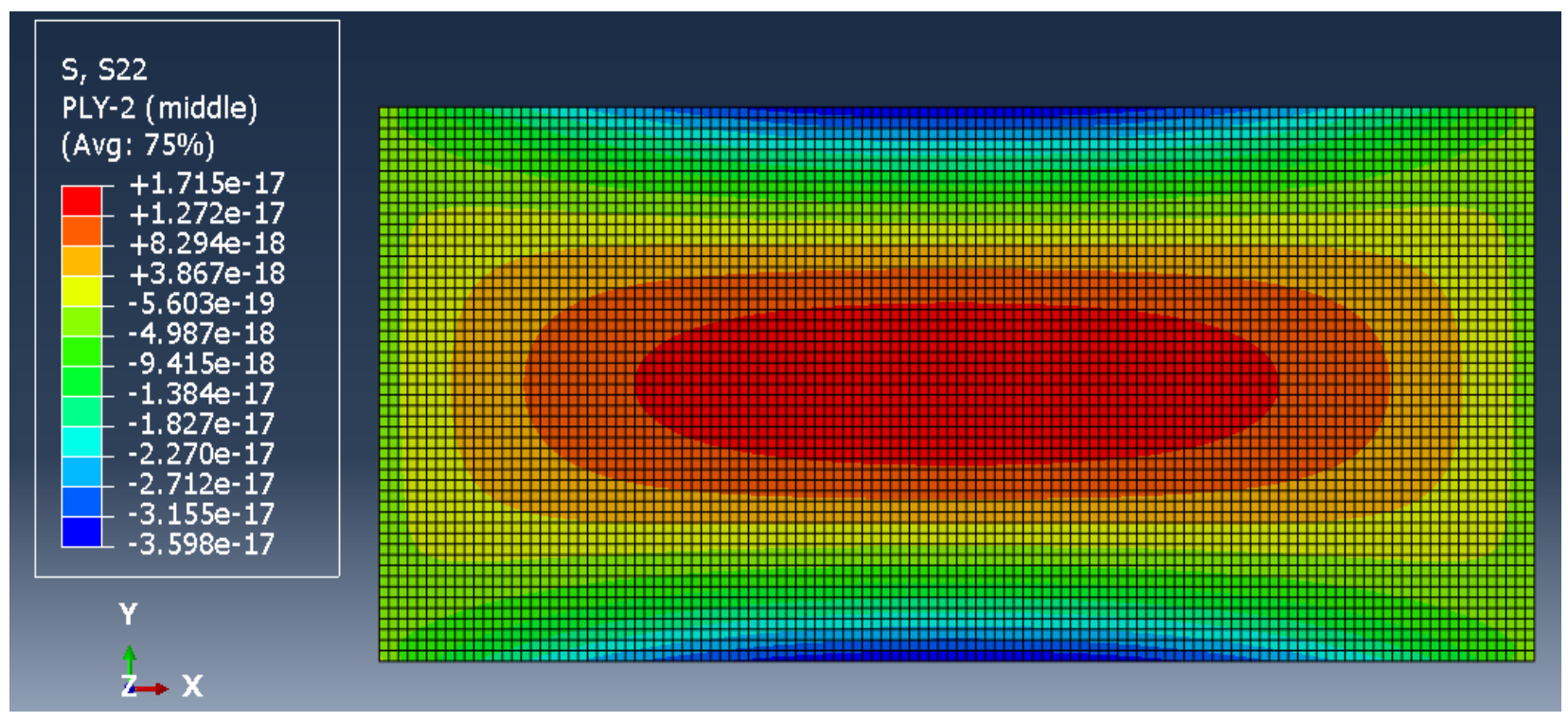

Figure 75: The in-plane normal stress, $\sigma_{\mathrm{yy}}=\sigma_{22}$, (MPa) on the core material

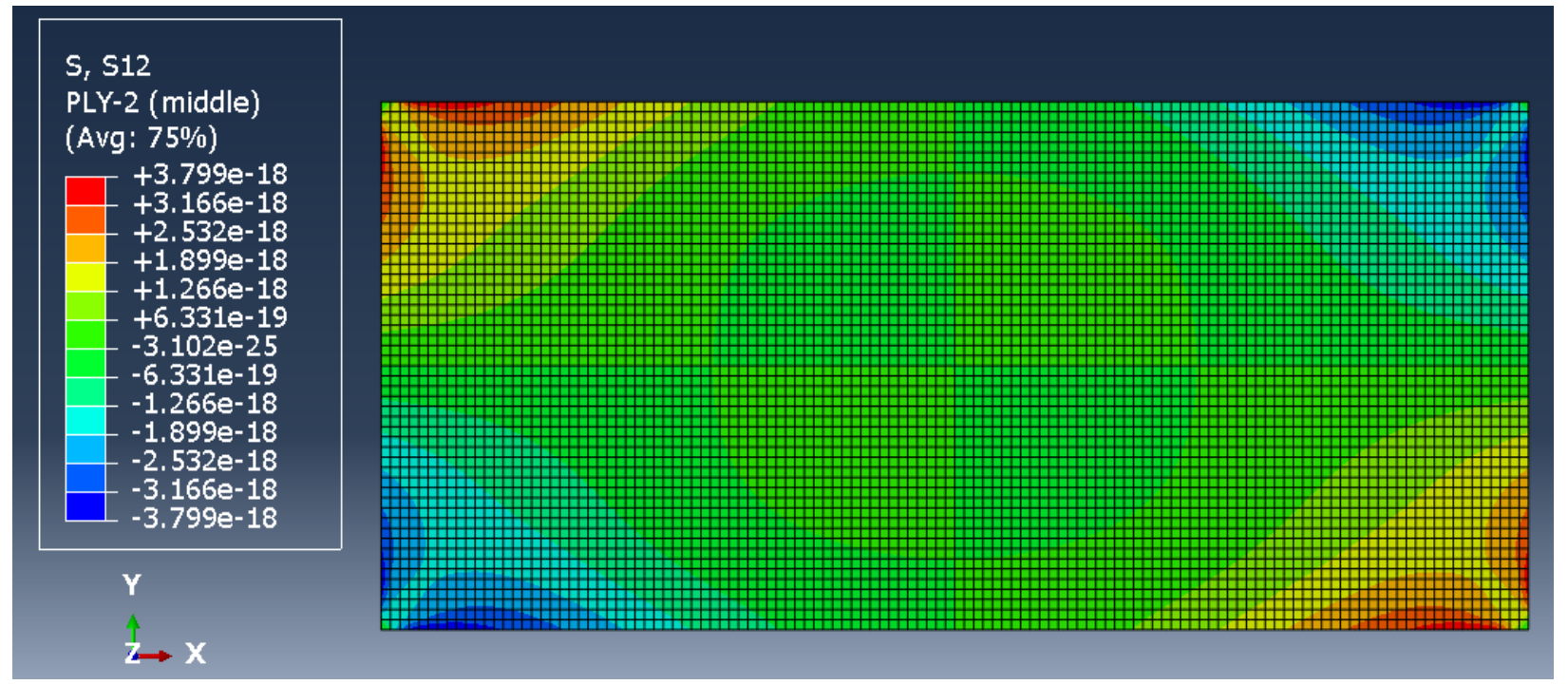

Figure 76: The in-plane shear stress, $\tau_{\mathrm{xy}}=\tau_{12},(\mathrm{MPa})$ on the core material 


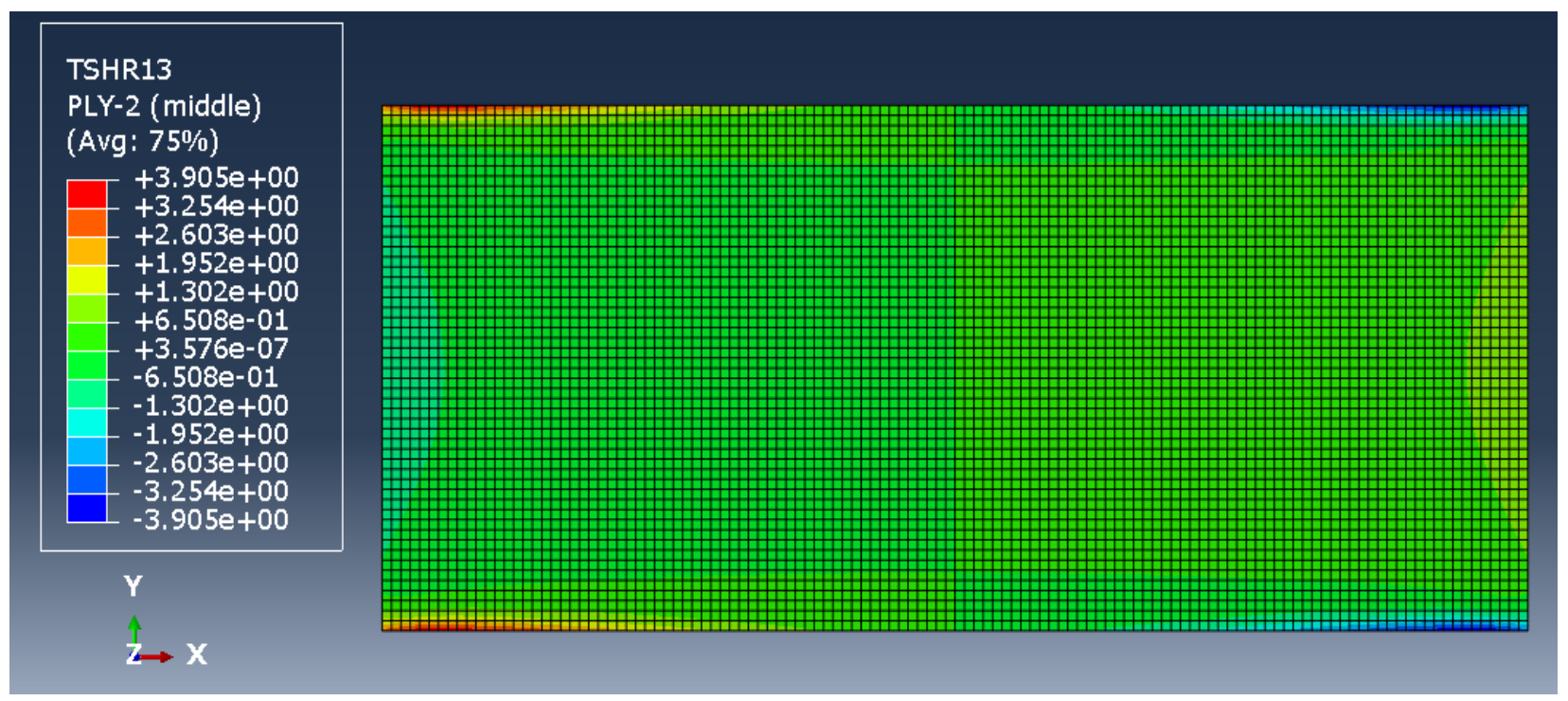

Figure 77: The transverse shear stress, $\tau_{x z}=\tau_{13},(\mathrm{MPa})$ on the core material

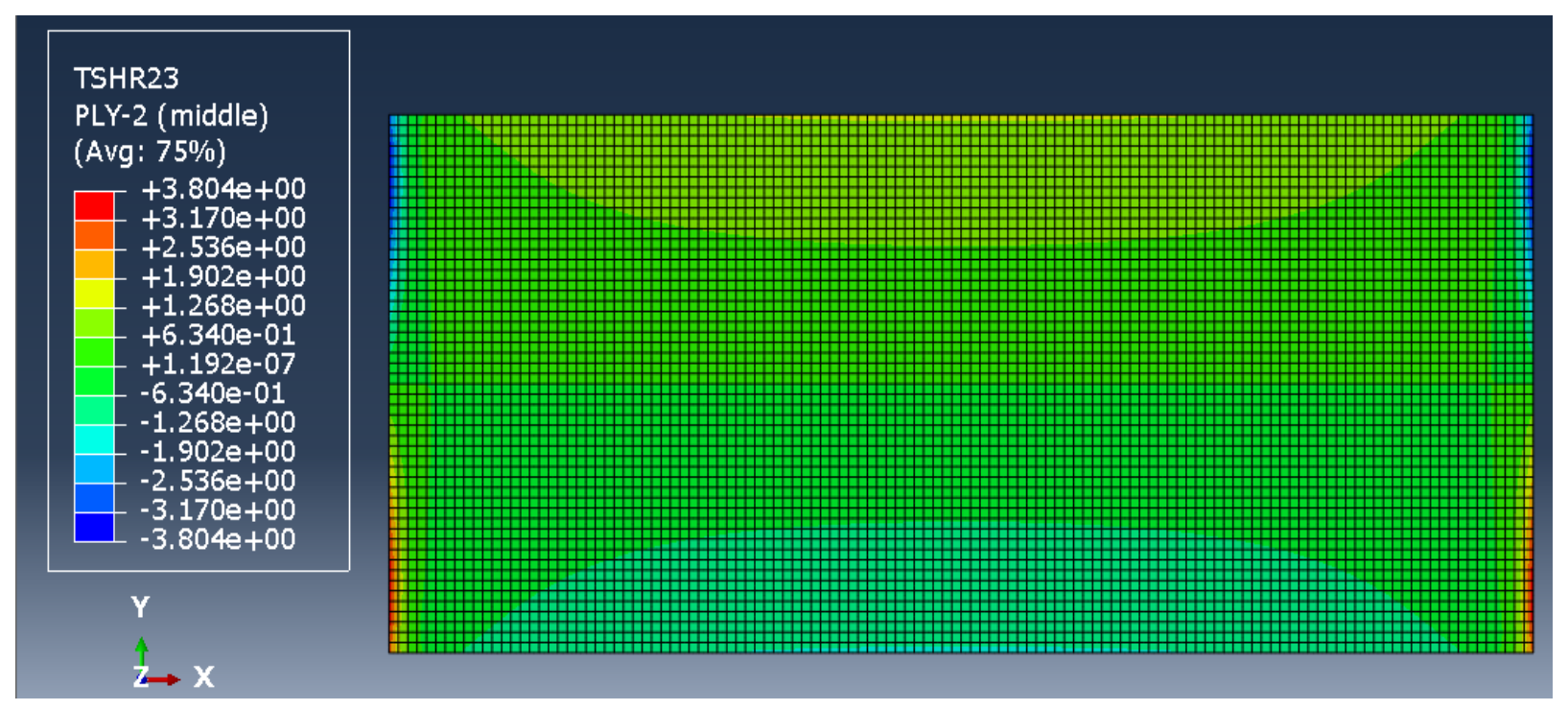

Figure 78: The transverse shear stress, $\tau_{\mathrm{yz}}=\tau_{23}$, (MPa) on the core material

\subsection{Validation Summary}

In this chapter, the proposed design was evaluated by FE analysis to ensure that no failure would occur in the structure. This was started with introducing different FE 
modeling techniques for sandwich panels. Different 2D models including global model, composite shell and layup (layered) model were selected as the main choices because of their higher computational efficiency. These models were analyzed and compared with the analytical results. The strengths and weaknesses of each model were discussed, and it was concluded that for the detailed design the layered model is the most suitable one, as it has the capability to present the local behavior in all layers in the structure. This was followed by the failure analysis of the face sheets and core material. Finally, it was concluded that both face sheets and the core would not fail under the stated loading conditions and the proposed design is a safe design, which can be evaluated further to be used in larger scale design of the whole railcar body. 


\section{Chapter 6. Conclusions and Recommendations}

This chapter summarizes the conclusions established for this study and suggestions for the future research works. It starts with summarizing the proposed methodology and obtained results and follows with recommendations for future studies.

\subsection{Conclusions}

The research methodology and conclusions drawn from this study can be summarized as below:

- The goal of the project was to reduce fuel consumption and GHG emission in the rail sector. This could be achieved through different approaches like improving the aerodynamics, using hybrid propulsion system or weight reduction. However, weight reduction is known to be the one of the most effective method for fuel saving and reducing the emission. In fact, the challenge was to minimize the weight of freight cars, while maintaining their design requirements defined by the AAR [6].

- The selection of the railcar type was based on their usage frequency and number of cars used on line by the main operators like $\mathrm{CP}$ and $\mathrm{CN}$. The hopper cars are the most frequently used type of railcars in Canada, with the covered hopper cars in the first place and the open hopper cars in the second. A covered hopper car was selected as the case study but the design requirements of this type of railcars are very similar to the open hopper cars. Thus, the proposed design methodology can be easily adopted for open hopper cars as well. 
- For lightweight design, both structural optimization and material substitution were considered to achieve the most possible weight reduction.

- Among different material options, the focus was on the metallic materials. Since the North American rail industry has a long history in working with metallic materials, both steel and aluminum, and convincing such a conservative industry to use composite materials with their associated manufacturing costs and complexity in design would be difficult. Moreover, there are still researches going on about the comparison of composite materials and aluminum alloys in terms of energy saving and environmental friendliness during the product life cycle. In fact, it is not easy to determine which of the aluminum or composites will outperform the other.

- For material selection, stress analysis was performed on the whole railcar body with the loading conditions stated by the AAR [6]. The results of stress analysis were used in conjunction with other AAR material requirements, and it was concluded that the aluminum alloys could be interesting alternatives for conventional steel cars. Traditionally, aluminum series $5 \mathrm{xxx}$ and $6 \mathrm{xxx}$ have been used in the rail industry but these alloys could not satisfy the AAR requirements for the floor panel of the covered hopper cars. On the other hand, series $2 \mathrm{xxx}$ and 7xxx have high strength which could satisfy the requirements, but they have weldability problems and are susceptible to stress corrosion cracking. All these reasons led the research to a new generation of aluminum-lithium alloys called the third generation. These series of alloys are microstructurally stable, ductile, weldable, strong and light. All these factors made them interesting candidates as a 
new material in the cargo railcars. Finally, the Al 2099 was selected as an alternative for the conventional steel.

- The use of light weight Al $2099\left(\rho_{A l}=2630 \mathrm{~kg} / \mathrm{m}^{3}\right)$ instead of conventional steel $\left(\rho_{S t}=7850 \mathrm{~kg} / \mathrm{m}^{3}\right)$ resulted in $66.5 \%$ weight reduction on each wagon (underframe, supporting structures and roof excluded). This weight reduction and its potential fuel savings and emission reduction can compensate the higher cost of material in few years. Also, in near future, with the possible Governmental regulations and penalties on the allowable emission level for freight cars, the investment on new light materials would be even financially more justifiable.

- The sandwich construction technique was introduced to achieve even further weight reduction. The developed sandwich panel was a mid-plane symmetry panel which was made from the same material, i.e. Al 2099, on all layers including both face sheets and its core. Among different core topologies, the honeycomb and truss lattice cores were shown to over perform others. However, due to the tendency of honeycombs to trap moisture and their lack of multifunctionality, the octet-truss lattice was selected for the preferred core material. The main advantages with these types of lattices are their ease of manufacturing and isotropic behavior.

- Since different parts of the floor panel were under the same loading conditions, only one part of the full panel was selected as the case study. The sandwich panel was designed in both microscale and macroscale level, and optimized based on design requirements. The optimized sandwich design has face sheets with $0.8 \mathrm{~mm}$ 
thickness and a core material with the thickness of $23.4 \mathrm{~mm}$ and relative density $\left(\rho_{c} / \rho_{o}\right)$ of 0.07 .

- The structural techniques, i.e. both sandwich design and optimization, led to $53.75 \%$ further weight reduction on the floor panel compared with the original 7 mm steel panels used in hopper cars (The new base material was assumed to be $\mathrm{Al}$ 2099). The floor panel is responsible for almost one quarter of the total wagon weight, and thus, the new design can result in almost $12.5 \%$ weight reduction in the whole wagon.

- It has been stated that a $10 \%$ weight reduction per freight railcar corresponds to $5 \%$ energy saving, assuming $180 \mathrm{~kJ} /$ gross t-km [3]. In this research, by employing both optimized sandwich construction in the floor panel and the light Al 2099 material in the whole body, the weight of each wagon can be almost reduced by $70 \%$. This corresponds to $35 \%$ fuel savings considering the stated assumption.

\subsection{Suggestions}

This research was mainly focus on developing light weight design concepts applicable to the freight type railcars. However, there are still some areas that can be more deeply investigated to improve the archived results:

- As was discussed, aluminum alloys and composite materials are competing options for the choice of material in the freight cars. Here, the proposed sandwich panel was fully made of Al 2099. However, the performance of the panel might be improved by using a combination of composite and metallic materials in different sandwich layers instead of using a single material for all layers. This can 
be investigated by considering all possible material options including third generation Al-Li alloys, 5xxx and 6xxx series aluminum alloys, steel, glass fiber reinforced polymers and $\mathrm{PVC}$ to find the best possible combination for the face sheets and the core material. This requires a multi-objective design optimization by considering different performance factors like cost, stiffness, strength and weight. Of course, the material compatibility and environmental effects like humidity should be taken into account. The equations derived in this thesis can be used as a basis for future design optimization.

- In this thesis, the optimization was performed on the floor panel. The same procedure can be used for the side walls. However, one must be paid attention to minor differences. For example, the loading on side walls is a hydrostatic type loading and their boundary conditions are similar to the case where three edges are simply supported, and one edge is free.

- The cross-sectional shape of struts in the truss lattice was assumed to be fixed but this can also be considered as a design variable for lattice weight minimization. In fact, using hollow cross sections known to be effective in improving the structural attributes, while reducing the relative density. Nevertheless, the associated manufacturing costs of these arbitrary cross sections might be something that prohibits designers from such a shape optimization.

- In this research, the optimization was in fact, a size optimization on the floor panel but a further layout optimization can also be performed to find the best possible layout for both side and floor stiffeners and their minimum thicknesses. 
- The static type loading was considered in this study, but one might expand this and consider the dynamic loading as well, e.g. the case where the rail car is on the curvy path and centrifugal force appears. This can be done by using the SPH technique which was briefly discussed in this thesis. However, since this method is not introduced in the AAR, it would be better to use it for the final validation rather than as the basis of design.

- The multifunctionality of truss lattice cores can be more advantageous in passenger railcars as compared to freight type cars, as requirements like acoustic damping or energy absorption are more important in passenger trains. Thus, although this project was focused on freight railcars, the methodology can be applied to passenger railcars which are currently made of foam or honeycomb cores.

- Finally, the octet-lattice truss was selected because of its isotropic behavior and ease of manufacturing but there is no promise on its better performance over pyramidal and tetrahedral lattices in different types of loading. This can be investigated with an experimental study on all three different types and comparing their response under different loading conditions to determine the best possible option for bending or possible hydrostatic loading. Nevertheless, the performance of all bending-dominated lattices are similar to each other, and as long as one is interested in the overall performance of a railcar, this may not be a critical topic. 


\section{References}

[1] Statista, "Largest global emitters of carbon dioxide by country 2016", [Online], Available: https://www.statista.com/statistics/271748/the-largest-emitters-of-co2-inthe-world/

[2] J.G.J. Olivier, G. Janssens-Maenhout, M. Muntean, J.A.H.W. Peters, "Trends in global $\mathrm{CO}_{2}$ emissions: 2016 Report”, PBL Netherlands Environmental Assessment Agency; European Commission, Joint Research Centre, 2016.

[3] S. Al Attar, "Hopper Railcars: A Preliminary Study on the use of Fiber Reinforced Polymers in Structural Components", M.Sc. Thesis, Carleton University, Ottawa, ON, 2016.

[4] Environment and Climate Change Candida, "Green House Gas Emission by Canadian Economic Sector", [Online], Available: https://www.ec.gc.ca/indicateursindicators/default.asp?lang=en\&n=F60DB708-1

[5] H. Helms, U. Lambrecht and U. Hopfner, "Energy Savings by Light-Weighting: Final Report", Institute for Energy and Environmental Research, Heidelberg, Germany, January 2003.

[6] Manual of Standards and Recommended Practices, Section C - Part II: Design, Fabrication, and Construction of Freight Cars, Association of American Railroads, USA, 2011.

[7] Y. Yang et al., "Recycling of Composite Materials", Chemical Engineering and Processing Intensification, Vol. 51, pp. 53-68, 2012. 
[8] E. Witten, T. Kraus, M. Kühne, “Composites Market Report 2016: Market Developments, Trends, Outlook and Challenges", Federation of Reinforced Plastics, Germany, November 2016.

[9] P. Schwab Castella et al., "Integrating Life Cycle Costs and Environmental Impacts of Composite Rail Car-Bodies for A Korean Train”, The International Journal of Life Cycle Assessment, Vol. 14, pp. 429-442, 2009.

[10] Y. S. Song, J. R. Youn and T.G. Gutowski "Life Cycle Energy Analysis of FiberReinforced Composites", Composites: Part A, Vo. 40, pp. 1257-1265, 2009.

[11] J.S. Arora, Introduction to Optimum Design, 3rd ed., Elsevier, USA, 2012.

[12] P.W. Christensen and A. Klarbring, An Introduction to Structural Optimization, $1^{\text {st }}$ ed., Springer Netherlands, 2009.

[13] W. Sun et al., "Analysis of Modal Frequency Optimization of Railway Vehicle Car Body.", Advances in Mechanical Engineering, Vol. 8, (4), pp. 1-12, 2016.

[14] K. Jacobsen, K. Severson and B. Perlman, "Effectiveness of Alternative Rail Passenger Equipment Crashworthiness Strategies”, in Proceedings of JRC2006, 2006, pp. 121-129.

[15] D. Wennberg and S. Stichel, "Multi-Functional Design of a Composite High-Speed Train Body Structure”, Structural and Multidisciplinary Optimization, Vol. 50, pp. $475-488,2014$.

[16] D. Wennberg, S. Stichel and P. Wennhage, “Optimization of Sandwich Panels for The Load Carrying Structure of High-Speed Rail Vehicles” International Journal of Aerospace Lightweight Structures, Vol. 2, (1), pp. 19-40, 2012. 
[17] T. Kuczek "Application of Manufacturing Constraints to Structural Optimization of Thin-Walled Structures”, Engineering Optimization, Vol.48, (2), pp. 351-360, 2016.

[18] A.M. Harte, J.F. McNamara and I.D. Roddy, "A Multilevel Approach to The Optimization of a Composite Light Rail Vehicle Body shell”, Composite Structures, Vol. 63, (3), pp. 447-453, 2004.

[19] C. W. Hudson, J. J. Carruthers and A. M. Robinson, "Multiple Objective Optimizations of Composite Sandwich Structures for Rail Vehicle Floor Panels", Composite Structures, vol. 92, (9), pp. 2077-2082, 2010.

[20] M. Mrzygłod and T. Kuczek, "Uniform Crashworthiness Optimization of Car Body for High-Speed Trains", Structural Multidisciplinary Optimization, Vol. 49, (2), pp. 327-336, 2014.

[21] L. Liu and W. Wakeland, "Combining Optimizer and Metamodeling for Railcar Structural Optimization", Proceedings of the Institution of Mechanical Engineers, Part F: Journal of Rail and Rapid Transit, vol. 219, (1), pp. 1-10, 2005.

[22] "Railroad Performance Measures" [Online]. Available: www.railroadpm.org

[23] “CP: Key Metrics", [Online]. Available: http://investor.cpr.ca/keymetrics/default.aspx

[24] Alamy, Railroad truck, [Online]. Available: http://www.alamy.com/stock-photorailroad-truckfm55-20fig8-8-129543395.html

[25] BNSF Railways, Covered Hoppers, [Online]. Available: http://www.bnsf.com/shipwith-bnsf/ways-of-shipping/equipment/covered-hoppers.html

[26] Manual of Standards and Recommended Practices, Section C: Car Construction Fundamentals and Details, Association of American Railroads, USA, 2008. 
[27] Morton Rail Products, [Online]. Available: https://unityrailway.com/morton-railproducts/

[28] W. C. Y. Richard and G. Budynas, Roark's Formulas for Stress and Strain, $7^{\text {th }}$ ed., New York, USA: McGraw-Hill, 2002.

[29] Simulia, Dassault Systems, "Abaqus 6.14 documentation, Abaqus Analysis User's Guide”, [Online]. Available: http://abaqus.software.polimi.it/v6.14/index.html

[30] P.C.J. Hoogenboom, CIE 4143, Class Lecture, Topic: "Shell Analysis, Theory and Application: Handout 4", Delft University of Technology, Delft, Netherland, May. 9, 2017.

[31] O. Walker, "Covered Hopper Railcar", [Online]. Available: https://www.pinterest.ca/OsbornWalkerLtd/covered-hopper-railcar/?lp=true

[32] Code of Practice for Fatigue Design and Assessment of Steel Structures, BS7608, British Standards, 1993.

[33] M. F. Ashby and Knovel (Firm), Materials Selection in Mechanical Design, $3^{\text {rd }}$ ed., Burlington, MA: Butterworth-Heinemann, 2005.

[34] M. F. Ashby, "Multi-Objective Optimization in Material Design and Selection", Acta Materialia, Vol. 48, (1), pp. 359-369, 2000.

[35] Investment Mine, Metal prices, "1-year Aluminum prices and price charts", [online]. Available: http://www.infomine.com/investment/metal-prices/aluminum/1-year/

[36] Investment Mine, Metal prices, "1-year Magnesium prices and price charts", [Online], Available: http://www.infomine.com/investment/metalprices/magnesium/1-year/ 
[37] General Steel Buildings, “2018 steel price forecast”, [Online], Available: https://gensteel.com/steel-building-prices/forecast

[38] F. Czerwinski, "Controlling The Ignition and Flammability of Magnesium for Aerospace Applications", Corrosion Science, Vol. 86, pp. 1-16, 2014.

[39] J. R. Davis, Alloying: Understanding the Basics, USA: ASM international, 2001.

[40] Lincoln Electric Canada, “Aluminum: Experience in Application”, [Online], Available: http://www.lincolnelectric.com/en-ca/support/process-andtheory/Pages/aluminum-application-detail.aspx

[41] M. Skillingberg, J. Green, “Aluminum Applications in the Rail Industry”, Light Metal Age, pp.8-13, October 2007.

[42] ASM Aerospace Specification Metals Inc., “Technical Data”, [Online]. [Available]: http://asm.matweb.com

[43] AZO Materials, Articles, “Aluminum Alloy 8090”, [Online]. Available: https://www.azom.com/article.aspx?ArticleID=8789

[44] S. Sasabe, "Welding of 2000 Series Aluminum Alloy Materials", Welding International, vol. 26, (5), pp. 339-350, 2012.

[45] S. Shah and S. Tosunoglu, "Friction Stir Welding: Current State of the Art and Future Prospects", The16th World Multi-Conference on Systemics, Cybernetics and Informatics, Orlando, Florida, 2012. 
[46] P.L. Threadgill et al., "Friction Stir Welding of Aluminum Alloys", International Materials Reviews, Vol. 54, (2), pp. 49-93, 2009.

[47] F.C. Campbell, "Aluminum" in Elements of Metallurgy and Engineering Alloys, USA: ASM International, 2008, pp. 487-508.

[48] Total Materia, "Corrosion of Aluminum and Aluminum Alloys", [Online], Available: http://www.totalmateria.com/Article14.htm

[49] N.E. Prasad, A. Gokhale and R.J.H. Wanhill, Aluminum-Lithium Alloys: Processing, Properties, and Applications, Boston, MA: Butterworth-Heinemann, 2014.

[50] S.N. Grieshop and R.G. Buchheit, "Comparison of the Corrosion Behavior of High Strength Aluminum Alloys after Exposure to ASTM B117 Environment”, From Department of Materials Science and Engineering, Ohio State University, Columbus, OH, 2014, [Online], Available: http://hdl.handle.net/1811/60362

[51] S. Schlatter, "Improvements of Mechanical Properties in Aluminum-Lithium Alloys", Sixteenth Ruth \& Ted Braun Awards for Writing Excellence, pp. 31-46, Saginaw Valley State University, MI, USA, 2013.

[52] E. Lertora and C. Gambaro, "AA8090 Al-Li Alloy FSW Parameters to Minimize Defects and Increase Fatigue Life", International Journal of Metal Forming, Vol.3, (31), pp. 1003-1006, 2010.

[53] ARCONIC, “Aluminum Alloy 2055-T84 Extrusions”, [Online], Available: https://www.arconic.com/adip/catalog/AFE2055-factsheet.pdf 
[54] ALCOA Aerospace Technical Fact Sheet, “Alloy 2099-T83 and 2099-T8E67 Extrusions", [Online], Available:

https://www.arconic.com/hard_alloy_extrusions/catalog/pdf/alloy2099techsheet.pdf

[55] Battelle Memorial Institute. Columbus Laboratories et al., Metallic Materials Properties Development and Standardization (MMPDS): MMPDS-08, April 2013.

[56] J. Rubadue, "Inclusion of a New Alloy in the Metallic Materials Properties Development and Standardization (MMPDS) Handbook", Available from Battelle Memorial Institute, September 2015. [Online], Available: https://www.mmpds.org/wp-content/uploads/2015/03/MMPDS-Adding-anAlloy_Oct2015.pdf

[57] A.S. Herrmann, P.C. Zahlen and I. Zuardy, "Sandwich Structures Technology in Commercial Aviation" in Sandwich Structures 7: Advancing with Sandwich Structures and Materials, O.T. Thomsen et al. Ed., Netherlands: Springer, 2005, pp. $13-26$.

[58] J.R. Vinson, "Sandwich Structures: Past, Present, And Future" in Sandwich Structures 7: Advancing with Sandwich Structures and Materials, O.T. Thomsen et al. Ed., Netherlands: Springer, 2005, pp. 3-12. 
[59] M. F. Aly, K. T. Hamza and M. M. Farag, "A Materials Selection Procedure for Sandwiched Beams Via Parametric Optimization with Applications in Automotive Industry" Materials \& Design, vol. 56, pp. 219-226, 2014.

[60] H-L. Fan et al., "Mechanics of Advanced Fiber Reinforced Lattice Composites", Acta Mechanica Sinica, Vol. 26, (6), pp. 825-835, 2010.

[61] M. F. Ashby, “The properties of foams and lattices", Philosophical Transactions: Mathematical, Physical and Engineering Sciences, Vol. 364, (1838), pp. 15-30, 2006.

[62] V. Crupi, G. Epsato and E. Guglielmino, "Comparison of Aluminum Sandwiches for Lightweight Ship Structures: Honeycomb vs. Foam”, Marine Structures, Vol. 30, pp. 74-96, 2013.

[63] D.T. Queheillalt and H.N.G. Wadley, "Cellular Metal Lattices with Hollow Trusses", Acta Materiala, Vol. 53, pp. 303-313, 2006.

[64] G.W. Kooistra, V.S. Deshpande and H.N.G. Wadley, "Compressive Behavior of Age Hardenable Tetrahedral Lattice Truss Structures Made from Aluminum", Acta Materiala, Vol. 52, pp. 4229-4237, 2004.

[65] G.W. Kooistra and H.N.G. Wadley, "Lattice Truss Structures from Expanded Metal Sheet”, Material \& Design, Vol 28, pp. 507-514, 2007. 
[66] J. Wang et al, "On The Performance of Truss Panels with Kagome Cores", International Journal of Solids and Structures, Vol. 40, pp. 6981-6988, 2003.

[66] J.H. Joo and K.J. Kang, "Modified Metallic Octet Truss Cellular Cores for Sandwich Structures Fabricated by an Expanded Metal Forming Process", Journal of Sandwich Structures and Materials, Vol.12, pp. 327-349, 2010.

[67] H.G. Wadley, N.A. Fleck and A.G. Evans, "Fabrication and Structural Performance of Periodic Cellular Metal Sandwich Structures”, Composite Science and Technology, Vol. 63, pp. 2331-2343, 2003.

[68] L. Dong, V. Deshpande and H. Wadley, "Mechanical response of Ti-6Al-4V octettruss lattice structures", International Journal of Solids and Structures, Vol. 60-61, pp. 107-124, 2015.

[69] J. D. Renton, Elastic Beams and Frames, $2^{\text {nd }}$ ed., Chichester, England: Horwood Publishing Ltd., 2002.

[70] M. Elsayed and D. Pasini, "Multiscale Structural Design of Columns Made of Regular Octet-Truss Lattice Material" International Journal of Solids and Structures, Vol. 47, pp. 1764-1774, 2010. 
[71] J. Pilling, "Strength of Sandwich Structures", From Department of Materials Science and Engineering Michigan Tech, [Online], Available:

http://www.mse.mtu.edu/ drjohn/my4150/sandwich/sp2.html

[72] T. Bitzer, Honeycomb Technology, $1^{\text {st }}$ ed., Dublin, California: Chapman \& Hali, 1997.

[73] V.S. Deshpande, N.A. Fleck, M.F. Ashby, "Effective Properties of the Octet-Truss Lattice Material", Journal of the Mechanics and Physics of Solids, Vol. 49, pp. 17471769, 2001.

[74] Jack R. Vinson, The Behavior of Sandwich Structures of Isotropic and Composite Materials, Lancaster, PA, USA: TECHNOMIC Publishing Company, Inc. 1999.

[75] "Mechanic of material: Stain", eFunda Inc., [Online], Available: http://www.efunda.com/formulae/solid_mechanics/mat_mechanics/strain.cfm\#engstr ain

[76] SlideShare, [Online], Available: https://www.slideshare.net/Khawwam/rc-iitwowayslabs 
[77] Composite Materials Handbook - 17 (CMH-17), Volume 6. Structural Sandwich Composites, SAE International, USA, 2013.

[78] W. C. Youn, R. G. Budynas, Roark's Formulas for Stress and Strain, $8^{\text {th }}$ ed., USA: McGraw-Hill Education, 2011.

[79] S. Timoshenko, S. Woinowsky-Krieger, Theory of plates and shells, $2^{\text {nd }}$ ed., New York: McGraw-Hill, 1959.

[80] M.F. Ashby, A.G. Evans, N.A. Fleck, L.J. Gibson, J.W. Hutchinson and H.N.G. Wadley, Metal Foams: A Design Guide, $1^{\text {st }}$ ed., USA: Butterworth-Heinemann, 2000.

[81] R. A. Staal et al., "Predicting Failure Loads of Undamaged Sandwich Honeycomb Panels Subject to Bending," Journal of Sandwich Structures \& Materials, vol. 11, pp. 73-104, 2009.

[82] “Abaqus 6.10 documentation, Abaqus Analysis User's Guide”, Simulia, Dassault Systems, [Online]. Available: https://www.sharcnet.ca/Software/Abaqus610/Documentation/docs/v6.10/books/stm/ default.htm?startat=ch03s06ath86.html\#stm-elm-transshearshells 
[83] “ABAQUS 6.14, Benchmarks Guide”, NAFEMS Benchmarks: Composite tests, [Online]. Available:

http://abaqus.software.polimi.it/v6.14/pdf_books/BENCHMARKS.pdf 